The Pennsylvania State University

The Graduate School

Department of Physics

\title{
STRUCTURE OF EVENTS CONTAINING DIRECT PHOTON OR NEUTRAL PION IN HADRONIC INTERACTIONS
}

\author{
A Thesis in \\ Physics : \\ by \\ Sajan Easo \\ Submitted in Partial Fulfillment \\ of the Requirements \\ for the Degree of \\ Doctor of Philosophy
}

December 1989 


\section{Abstract}

Fermilab Experiment E706 studies quark and gluon interactions by analyzing the yield of $\pi^{0} \mathrm{~s}$, single photons and the accompanying hadrons in the collisions of charged pions and protons with nucleons. The photons emerging directly from the hard scattering of partons (direct photons) and the hadrons accompanying them provide information about the parton subprocesses. In this experiment the events were triggered by a large transverse momentum electromagnetic shower in a liquid argon calorimeter. The charged particle spectrometer consisted of a set of silicon strip detectors, a set of multiwire proportional chambers and a dipole magnet. The data alysis shows evidence for jet structure in the events which contain either a high transverse momentum (above $4 \mathrm{GeV} / \mathrm{c}$ ) single photon or neutral pion. After reconstructing the directions of these jets, a study of the properties of the hadrons in these jets and of the underlying parton level kinematics is done. In this thesis, an overview of the experiment is presented along with a detailed description of the hardware and software of the charged particle tracking system. This is followed by a discussion of the analysis of the jets in the events selected from the data from the first run of the experiment in 1987-88. 


\section{Contents}

$\begin{array}{ll}\text { List of Figures } & \text { ix }\end{array}$

List of Tables $\quad \mathrm{xv}$

Acknowledgments $\quad$ xvi

Chapter 1 Introduction 1

1.1 Direct photon production $\ldots \ldots \ldots \ldots \ldots$

1.2 Physics motivation of the experiment $\ldots \ldots \ldots \ldots$

1.3 Kinematics of hard scattering $\ldots \ldots \ldots \ldots \ldots$

1.4 Correlations in the event structure $\ldots \ldots \ldots \ldots$

1.5 E706 experimental program $\ldots \ldots \ldots \ldots \ldots$

1.6 Variables and notations used . . . . . . . . . 11

1.6.1 Cross sections . . . . . . . . . . . . 18

1.7 Outline of the thesis $\ldots \ldots \ldots \ldots \ldots$

Chapter 2 Experimental Setup 21 
2.1 Overall layout . . . . . . . . . . . . . . . . . 21

2.2 Beam line and veto walls . . . . . . . . . . 21

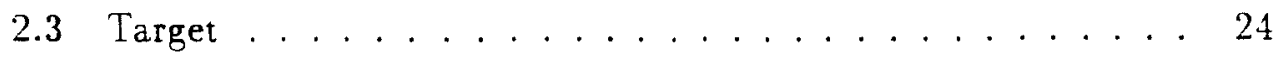

2.4 Charged particle spectrometer . . . . . . . . . 25

2.4 .1 Silicon strip detector $\ldots \ldots \ldots \ldots \ldots$

2.4.2 Multiwire proportional chamber system . . . . . . 31

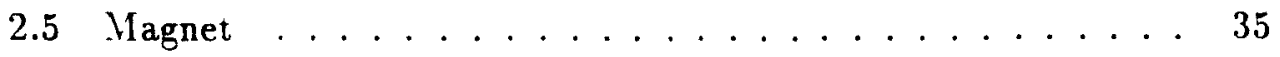

2.5 .1 Characteristics . . . . . . . . . . 35

2.6 Liquid argon calorimeter . . . . . . . . . . . 35

2.6 .1 General description . . . . . . . . . . 35

2.6.2 The electromagnetic calorimeter (EMLAC) . . . . 36

2.6.3 The hadron calorimeter (HALAC) . . . . . . . 44

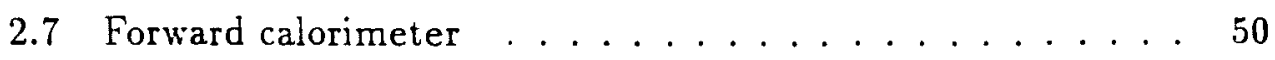

2.8 E672 and muon detection $\ldots \ldots \ldots \ldots \ldots \ldots$

Chapter 3 E706 Multiwire Proportional Chambers 54

3.1 Construction of the E706 MWPC's . . . . . . . . 54

3.1.1 General construction considerations . . . . . . . 54

3.1.2 Frames for cathodes and anodes . . . . . . . 54

3.1.3 Cathode construction . . . . . . . . . . 55

3.1.4 Anode construction . . . . . . . . . . . . 58

3.1.5 Spacing between the various planes . . . . . . 61

3.2 Operation of the E706 MWPC's . . . . . . . . 6 61 
3.2.1 Readout electronics . . . . . . . . . . . . 63

3.2.2 Gate width and readout delays . . . . . . . . 63

3.2.3 Read-out electronics . . . . . . . . . . . 64

Chapter 4 Trigger and Data Acquisition 66

4.1 Description of the trigger system ........... 66

4.1.1 Beam and interaction definition ........ 66

4.1.2 Preselection of an interaction ......... 67

4.1.3 Final selection of the event . . . . . . . . . 69

4.2 Data acquisition System (D AQ) . . . . . . . . 71

4.2.1 Data flow ................. 71

4.3 Monitoring data . . . . . . . . . . . . 71

4.4 Amount of data collected ............. 73

$\begin{array}{ll}\text { Chapter } 5 \text { Charged Particle Reconstruction } & \mathbf{7 5}\end{array}$

5.1 Charged particle tracking. . . . . . . . . . 75

5.1.1 General philosophy of downstream tracking .... . 75

5.1.2 General philosophy of upstream tracking . . . . . 80

5.1 .3 Beam tracking. .............. 87

5.1.4 Linking at the magnet ............ 87

5.1.5 Philosophy of vertex reconstruction . . . . . . 97

5.1 .6 Relinking ................. 106 
5.1.7 Determination of momentum and charge . . . . . 109

2 Detector simulation . . . . . . . . . . . . . . 112

3 Efficiency of the tracking system . . . . . . . . . 113

pter 6 Electromagnetic Shower Reconstruction $\quad 120$

.1 Group and peak finding in a given view . . . . . . . . 120

.2 Shoulder finding in a peak . . . . . . . . . . . . 122

$.3 \gamma$ reconstruction . . . . . . . . . . . . . . . . . 122

.4 Correlation of $\gamma$ s in $\mathbf{r}$ and $\phi$ view . . . . . . . . 125

ipte 7 Data Reduction and Event Classification $\quad 127$

i1 Event selection . . . . . . . . . . . . 127

¡.2 Shower selection . . . . . . . . . . . . . . . 129

¡.3 Charged track selection . . . . . . . . . . . . . . . 129

i.4 $\pi^{0}$ event selection . . . . . . . . . . . . . . 130

i.5 Direct photon candidate selection ........... 136

7.6 Event Analysis ................... 136

apter 8 Analysis of Jet Events 138

8.1 Evidence of jet structure . . . . . . . . . . . . 138

8.2 Jet reconstruction . . . . . . . . . . . . . . 143 
8.3 Results of jet reconstruction . . . . . . . . . . . 147

8.4 Parton distributions ............... . . 150

8.5 Fragmentation functions ............. 163

9 Conclusions $\quad 176$

$\begin{array}{lr}\text { Chapter } 9 \text { Conclusions } & 176\end{array}$

9.1 Construction and performance of the apparatus . . . . 176

9.2 Remarks on the analysis of the data . . . . . . . 176

$\begin{array}{ll}\text { Appendix A Magnetic Field Parameterization } & 179\end{array}$

A.1 Effective field ................. 183

Appendix B Physics Principles of MWPC's 185

B.1 Interaction of a charged particle in a gaseous medium . . . 185

B.2 Geometry of MWPC . . . . . . . . . . . 186

B.3 Electrostatics of MWPC .............. 186

B.4 Dynamics of MWPC . . . . . . . . . . . . 192

B.5 Timing properties ................. 193

B.6 Energy loss due to electromagnetic interactions . . . . . 196

B.7 Gas used for MWPC . . . . . . . . . . . . 199

$\begin{array}{lr}\text { Bibliography } & 202\end{array}$

viii 


\section{List of Figures}

1.1 QCD Compton and annihilation diagrams ........ 3

1.2 Ratio of Direct photon cross sections from $\pi^{+}$and $\pi^{-}$beams 5

1.3 Two-body hard scattering process ............ 7

$1.4 \gamma \gamma$ mass plot. . . . . . . . . . . . . . . . 12

$1.5 d \sigma / d p_{T}$ for $\pi^{0}$ 's in the process $\pi^{-}+B e \rightarrow \pi^{0}+X \ldots 13$

$1.6 d \sigma / d p_{T}$ for $\gamma^{\prime}$ s in the process $\pi^{-}+B e \rightarrow \gamma+X \ldots 14$

1.7 Ratio of $\gamma / \pi^{0}$ versus $p_{T} \ldots \ldots \ldots \ldots$

8 Plot of structure functions . . . . . . . . . . 20

2.1 Picture of MWEST . . . . . . . . . . . . 22

2.2 E706 coordinate system . . . . . . . . . . 23

2.3 Layout of the Silicon Strip Detector(SSD) . . . . . . 28

2.4 Typical event as seen in the SSD's . . . . . . . . 29

2.5 Planes of MWPC . . . . . . . . . . . . 33

2.6 Picture of completed chambers . . . . . . . . . . . 34

2.7 The liquid argon calorimeter ............. 37

2.8 Electromagnetic calorimeter: $\mathrm{r}$ and and $\phi$ strips . . . . . 38

2.9 R strips and focussing effect . . . . . . . . . . 40 
2.10 Energy difference in $\mathrm{R}$ and $\phi$ of electrons at (a) $25 \mathrm{GeV} / \mathrm{c}$ (b) $50 \mathrm{GeV} / \mathrm{c}$ (c) $100 \mathrm{GeV} / \mathrm{c} \ldots \ldots . \ldots 43$

2.11 Energy uncertainty versus energy of electrons . . . . . . . . 45

2.12 Difference in the location of electromagnetic showers and the projection of charged tracks to the front of EMLAC . . . . 46

2.13 Picture of the triangular pads . . . . . . . . . . . . . 48

2.14 Picture of the forward calorimeter . . . . . . . . . . 52

2.15 E672 MWPC, toroid, dimuon event picture . . . . . . . 53

3.1 Picture of a completed cathode . . . . . . . . . 56

3.2 Garlands . . . . . . . . . . . . . . . . . . 59

3.3 Laying wires . . . . . . . . . . . . . . . . 62

3.4 Typical MWPC plateau curves ............ 65

4.1 Data acquisition flow ............... 72

5.1 Residues from a straight line fit for plane $20 \ldots 81$

$5.2 \chi^{2}$ for 16 hit tracks ................. 82

$5.3 \chi^{2}$ for 15 hit tracks ................. 83

$5.4 \chi^{2}$ for 14 hit tracks .................. 84

$5.5 \quad \chi^{2}$ for 13 hit tracks ............... 85

5.6 Relative multiplicity of $16,15,14$ and 13 hit tracks . . . 86

5.7 A track in the magnetic field ............ 89

5.8 Angles involved in the magnetic field corrections . . . . 9 90 
5.9 Field corrections in projections to magnet center versus their expected magnitudes in the $\mathrm{X}$ view . . . . . . . . 93

5.10 Field corrections in projections to magnet center versus their expected magnitudes in the $\mathrm{Y}$ view $\ldots \ldots . . . . .99$

5.11 Field correction in $\mathrm{Y}$ view bend angle versus its expected magnitude ...................... 95

$5.12 \Delta x$ at the magnet center . . . . . . . . . . . 98

5.13 $\Delta y$ at the magnet center ................. . . 99

$5.14 \Delta$ slope $\mathrm{y}$ at the magnet center . . . . . . . . . 100

5.15 Impact parameter distributions for SSD tracks in the $\mathrm{X}$ view 103

5.16 Impact parameter distributions for SSD tracks in the $\mathrm{Y}$ view 104

5.17 Impact parameter distribution for MWPC tracks in the $\mathrm{Y}$

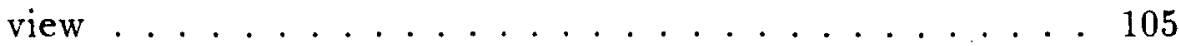

$5.18 \mathrm{Z}$ coordinate of the matched vertices . . . . . . . 107

$5.19 V_{z}$ difference in the two views ........... . . 108

5.20 Dimuon $\left(\mu^{+} \mu^{-}\right)$mass in GeV $/ c^{2} \ldots \ldots 111$

5.21 Efficiency of plane number $20 \ldots 115$

5.22 Generated versus Reconstructed momenta . . . . . . . 117

5.23 Fraction of the number of tracks linked in the events $(A)$ in $\mathrm{X}$ view $(\mathrm{B})$ in $\mathrm{Y}$ view ................. 118

$5.24 \frac{\Delta P}{P}$ versus $\mathrm{P}$ for charged tracks . . . . . . . . . 119

7.1 Asymmetry of pizeros . . . . . . . . . . . . . 132

7.2 Illustration of directionality ............. 134 
T.3 $p_{T}$ versus directionality of showers $\ldots \ldots \ldots$. . . . . 135

$8.1 p_{T}$ vectors . . . . . . . . . . . . . . 140

8.2 Rapidity versus azimuthal angle . . . . . . . . . . . 141

8.3 Azimuthal difference between particles in the event . . . . 142

8.4 Rapidity difference between leading and next leading particles........................ . . 144

8.5 (A) Rapidity of generated versus reconstructed jets; (B) Azimuthal angle of generated versus reconstructed jets . . . . . 148

8.6 (A) $\phi$ of leading particle versus that of recoil jet; (B) Rapidity of leading particle versus that of recoil jet . . . . . . . 149

8.7 Rapidity distribution of trigger jet in single $\gamma$ events . . . . . 151

8.8 Rapidity distribution of recoil jet in single $\gamma$ events . . . . 152

8.9 Recoil jet multiplicity in $\pi^{0}$ events in $\pi^{-}$data . . . . . 153

8.10 Recoil jet multiplicity in $\pi^{0}$ events in proton data . . . . . 154

8.11 Recoil jet multiplicity in $\gamma$ events in $\pi^{-}$data . . . . . . 155

8.12 Recoil jet multiplicity in $\gamma$ events in proton data . . . . . 156

8.13 Trigger jet multiplicity in $\pi^{0}$ events in $\pi^{-}$data . . . . . 157

8.14 Trigger jet multiplicity in $\pi^{0}$ events in proton data . . . . 158

8.15 Trigger jet multiplicity in $\gamma$ events in $\pi^{-}$data $\ldots \ldots 159$

8.16 Trigger jet multiplicity in $\gamma$ events in proton data . . . . 160

8.17 Angle of recoil particles with respect to recoil direction . . . 161

8.18 Transverse momentum of recoil particles with respect to recoil direction . . . . . . . . . . . . . . . 162 
$8.19 x_{a}$ and $x_{b}$ for $\gamma$ events in proton beam ......... 164

$8.20 x_{a}$ and $x_{b}$ for $\gamma$ events in $\pi^{-}$beam ........... 165

$8.21 x_{a}$ and $x_{b}$ for $\pi^{0}$ events in proton beam ........ 166

$8.22 x_{a}$ and $x_{b}$ for $\pi^{0}$ events in $\pi^{-}$beam .......... 167

$8.23 x_{a}$ and $x_{b}$ for MC events in pp .............. 168

8.24 Difference between $x_{a}$ and $x_{b}$ for $\gamma$ events in proton beam 169

8.25 Momentum fraction of particles in the recoil jet for single $\gamma$ events in $(A)$ proton data and a parameterization function

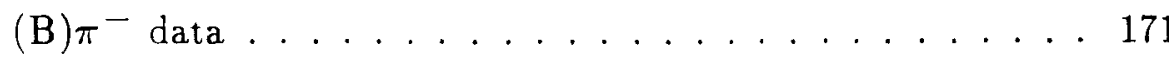

8.26 Momentum fraction of particles in the recoil jet for $\pi^{0}$ events in (A) proton data $(B) \pi^{-}$data $\ldots \ldots . \ldots 172$

8.27 Momentum fraction of particles in the recoil jet for proton beam in the Monte Carlo data . . . . . . . . . . . 173

8.28 Charge ratio in the recoil jet for $\gamma$ events in $(A)$ proton data (B) $\pi^{-}$data ......................... 174

8.29 Charge ratio in the recoil jet for $\pi^{0}$ events in $(A)$ proton data (B) $\pi^{-}$data ............................ 175

A.1 $B y$ component of the magnetic field versus $z \ldots 181$

A.2 $B_{z}$ component of the magnetic field versus $z \ldots \ldots 182$

B.1 Electric field equipotential lines inside the MWPC . . . . 189

B.2 Effect of mechanical displacement of wires . . . . . . . 190

B.3 Multiplication factor as a function of $V_{0} / V_{T} \ldots \ldots 194$

B.4 MWPC timing properties .............. 195 
B.5 Timing distribution of charge collection . . . . . . 197 


\section{List of Tables}

2.1 Types and characteristics of targets .......... 25

2.2 Properties of the SSD's . . . . . . . . . . . 30

2.3 Dimensions of MWPC ................. 32

3.1 Typical voltages for MWPC operation . . . . . . . . 64

4.1 Typical threshold setting for triggers . . . . . . . . . 69

4.2 Number of triggers collected . . . . . . . . . . 74

5.1 Efficiency of MWPC planes ............. 114

7.1 Numbers of events of various types used in the analysis . . . 137 


\section{Acknowledgments}

There are many people who have contributed directly and indirectly to the completion of this thesis. I would like to thank all the faculty members of E706 for all the invaluable guidance and support they have provided over the years. I would like to thank all the graduate students and postdoctoral fellows of E706 for all the collaboration, encouragement and suggestions I have received.

I would like to thank John Passaneau for the help during the construction of the apparatus. I would like to express my gratitude to Jerri Ann Zitek for the assistance over the years. I would like to thank the Fermi National Accelerator Laboratory and the Pennsylvania State University for all the facilities and opportunities provided. 


\section{Chapter 1}

\section{Introduction}

\subsection{Direct photon production}

This experiment (Fermilab experiment E706) studies quark and gluon interactions by analyzing the yield of $\pi^{0}$ 's and single direct photons and the accompanying hadrons in collisions of $\pi^{-}, \pi^{+}$and protons with nucleons. In Quantum Chromodynamics (QCD), the term partons refers to the constituents of colliding high energy particles. The term direct photon or prompt photon refers to a photon emerging directly from the hard scattering of partons. The interest in the direct production of photons [19] stems from the fact that a substantial yield of photons at large transverse momentum would allow a direct probe of parton subprocesses. By virtue of their elementary nature. such photons can emerge as free particles with all the transverse momentum imparted to them in the primary collision. Hence the direct photons provide pristine information about the hard scattering and provide a crucial test for the theory of QCD. 


\subsection{Physics motivation of the experiment}

In a parton level subprocess in which a strongly coupled vector gluon is emitted from a quark, the gluon can be replaced by the electromagnetically coupled photon $[24]$. The photon emission probability relative to the gluon yield is reduced essentially by the ratio of $\alpha / \alpha_{s}$ (where $\alpha$ is the fine structure constant and $\alpha_{S}$ is the strong coupling constant). Figure 1.1 shows the leading order contributions to direct photon production at large transverse momentum where a perturbative expansion in QCD is expected to be valid. The first two diagrams in Figure 1.1, which are the QCD analog of Compton scattering, are commonly referred to as the Compton diagrams while the second set of diagrams in Figure 1.1 are referred to as the annihilation diagrams.

From these figures one sees that when a photon appears in the final state, either the hard scattering is initiated by a gluon or the photon is accompanied by the gluon. In the absence of photon Bremsstrahlung (a photon being radiated by a quark or gluon) there are only these two hard scattering subprocesses for producing direct photons. If the contributions from these leading order diagrams can be isolated, prompt photon production can be used to extract vital information on either the gluon structure functions 3 .

of the pion and the nucleon from rates extracted for the Compton process or the gluon fragmentation function to hadrons from the annihilation process.

In p-nucleon and $\pi^{+}$-nucleon collisions the Compton process dominates. If only the Compton term is contributing, the ratio of the direct photon cross 


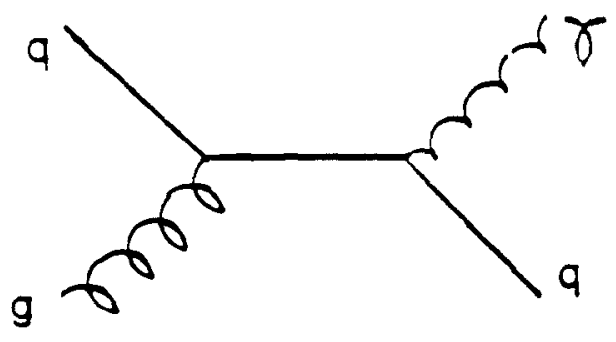

Compton Diagrams .

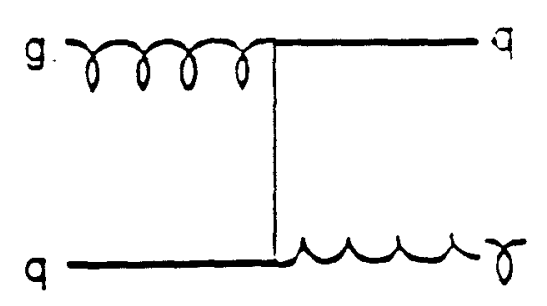

$\frac{d \dot{\sigma}}{d \dot{t}}=\frac{-e_{q}^{2} \pi \alpha \alpha_{9}}{3 \dot{j}^{2}}\left(\frac{\dot{u}}{\hat{j}}-\frac{\dot{j}}{\dot{u}}\right)$

gluon structure
function
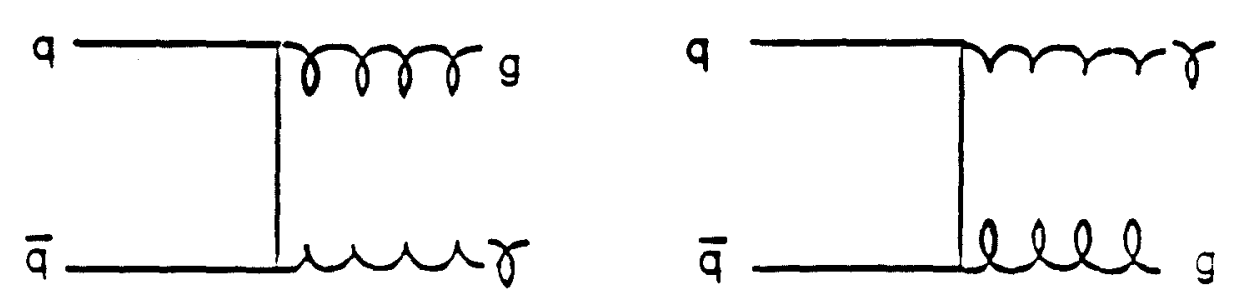
gluon fragmentati
function

Annihilation Diagrams - $\quad \frac{d \dot{\sigma}}{d \dot{t}}=\frac{8 e_{q}^{2} \pi \alpha \alpha s}{9 \dot{s}^{2}}\left(\frac{\dot{u}}{\hat{t}}+\frac{\dot{t}}{\dot{u}}\right)$

Figure 1.1: QCD Compton and annihilation diagrams 
section from $\pi^{-}$and $\pi^{-}$beams would be equal. However. the presence of the $\bar{u}$ quark in $\pi^{-}$as opposed to $\bar{d}$ in $\pi^{-}$means that the annihilation subprocess will make a relatively larger contribution in the $\pi^{-}$beam case $[11]$.

This follows both from the larger charge for the $\bar{u}$ quark as well as from the fact that the $u$ quark distribution is harder than that of the $d$ quark. At low $p_{T}$ the gluon distribution is more dominant than the quark distribution and hence the Compton process dominates. As a consequence of the charge invariance of strong interactions, the yield of direct photons via the Compton process are same for the $\pi^{-}$and $\pi^{-}$reactions. As the $p_{T}$ increases the annihilation process makes an increasingly more important contribution. Hence at low $p_{T}$, the ratio of the direct photon cross section from a $\pi^{+}$ beam to that from a $\pi^{-}$beam is near unity, but at higher $p_{T}$ this ratio decreases. Figure $1.2[6]$ shows this effect. Hence, the difference in the yield of detected direct photons in these two reactions can be attributed to the annihilation process.

\subsection{Kinematics of hard scattering}

Figure 1.3 shows the schematic representation of the underlying kinematics of a two-body hard scattering process. The parton a in hadron $\mathrm{A}$ interacts with parton $b$ in hadron $B$ to give rise to partons $c$ and $d$. The partons $\mathrm{c}$ and $\mathrm{d}$ undergo hadronisation and thereby several hadrons are produced. of direct photon production one of the partons created is to be replaced by 


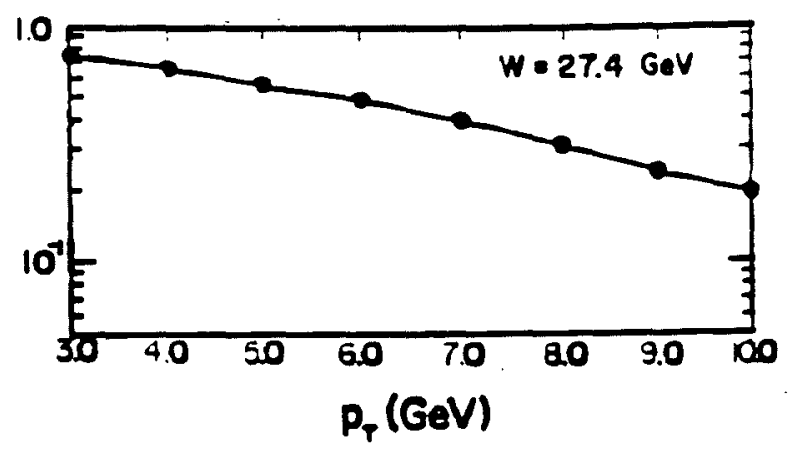

Figure 1.2: Ratio of Direct photon cross sections from $\pi^{+}$and $\pi^{-}$beams 
the photon. For the Compton process the other parton created would be a quark and for the annihilation process the other parton produced would be a gluon.

The jet which the experiment triggers on is called the trigger jet. The other jet created is called the recoil jet or the away-side jet. Similarly the beam jet and the target jet refer to the jets associated with beam and target particles, respectively. In the later part of this chapter the kinematics of parton scattering for direct photon production are explored.

\subsection{Correlations in the event structure}

Several phenomenological investigations $\{12,13\}$ have emphasized the desirability of measuring the correlations between direct photons and their associated jets of hadronic particles. The subject of correlations between the jets produced is very interesting for several reasons, some of which are described below.

1. The simple structure which exists at the lowest order of perturbation theory makes several striking predictions for the correlations between the trigger photon and associated high $p_{T}$ hadrons. These can be used to test the validity of the QCD description of the process of direct photon production.

2. Simultaneous measurement of the four-vectors of the trigger photon and the recoiling away-side jet allows the underlying parton-level kine- 


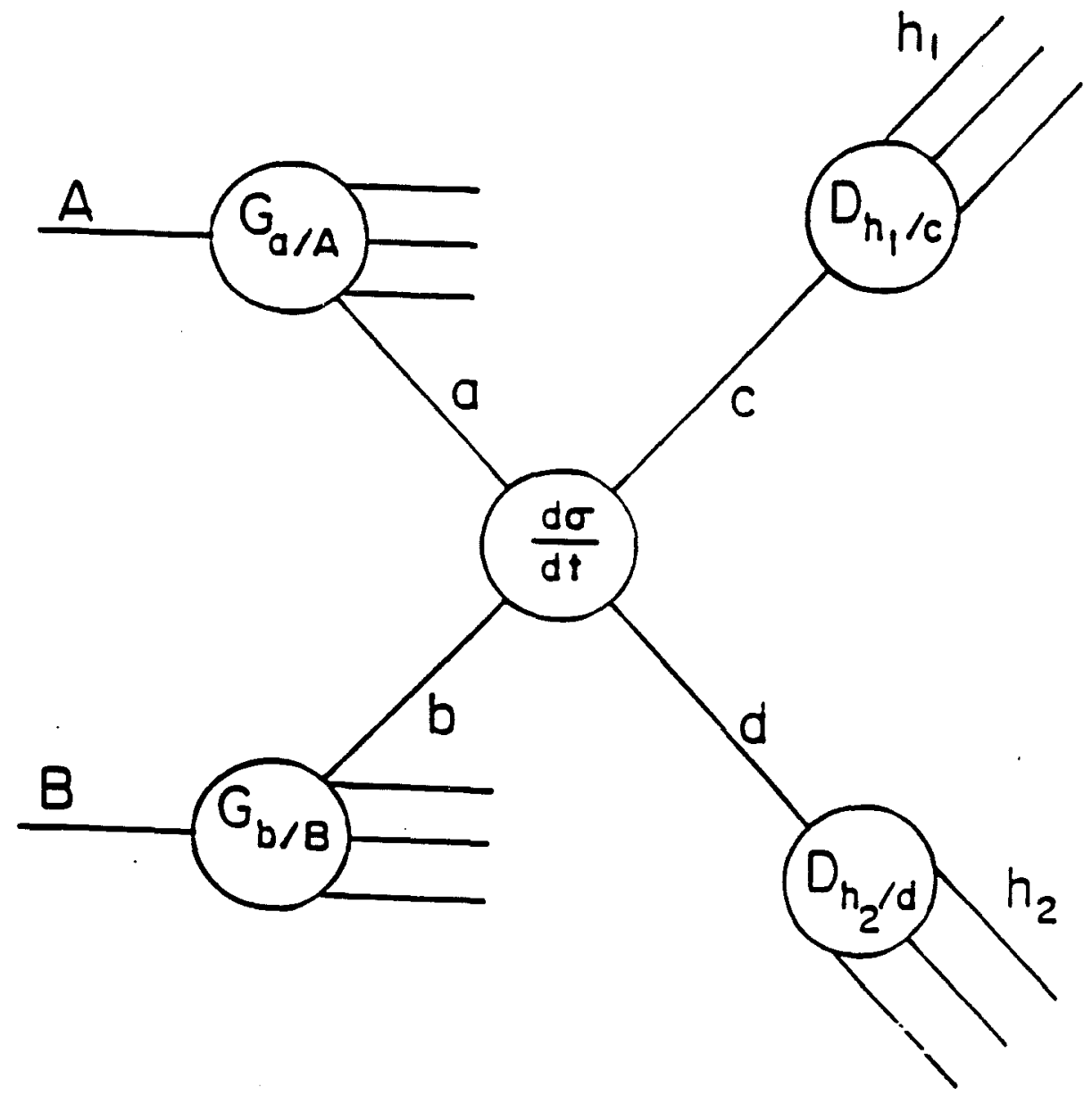

Figure 1.3: Two-body hard scattering process 
matics $[25]$ to be calculated in the case of single direct photon production. This can be used to provide constraints on and make measurements of various parton distribution and fragmentation functions.

3. The jets recoiling from $\pi^{0}$ s or from charged hadrons can often be initiated by gluons. Hence a comparison of away-side jets that accompany $\gamma$ 's with those accompanying $\pi^{0}$, s and hadrons could yield at least a qualitative measure of the difference between quark and gluon fragmentation.

As a first example, the case of the correlations between the trigger photon and hadrons in the same-side jet are considered. A process which could give rise to production of particles near a photon is a non-direct photon process like the Bremsstrahlung process. One of the high $p_{T}$ scattered partons radiates a hard photon and the remainder fragments into hadrons in the usual way. This contribution to the photon yield partially obscures the simpler direct photon contributions from the leading order diagrams. Identifying such events with a high $p_{T}$ Bremsstrahlung photon is difficult. since in order for this to happen, the photon should take most of the parton's momentum and therefore the acompanying hadrons would have small $p_{T}$. As a result, it would be difficult to isolate them from the beam and target remnants. In addition, the fragmentation process would give hadrons some transverse momentum with respect to the original parton direction of motion. When this is combined with the fact that these hadrons have a relatively small longitudinal momentum fraction, it becomes quite possible 
that there is a sizable angular separation between the photon and associated hadrons. Thus the photon in a Bremsstrahlung event may appear to be isolated from the hadrons in the event.

As another example, the correlations between the trigger photon and hadrons which are on the away side are considered. If these hadrons have sufficiently large $p_{T}$, they can be interpreted as having resulted from the recoiling parton. Since the photon couples to electric charge, the expectation for the Compton process is that the recoiling quark will predominantly be a u quark. In proton-proton collisions the ratio of $u$ to $d$ quarks on the awayside will be approximately 8 , with a factor of 4 coming from the square of the quark charge and a factor of 2 coming from the fact that there are two valence $u$ quarks and one valence $d$ quark in a proton. This ratio may give rise to a large ratio of positive to negative hadrons on the away-side if, as expected, a u quark yields on the average more positive hadrons than a d quark. Furthermore, for a fixed value of the trigger $p_{T}$ this fraction should increase with awayside $p_{T}$ since, as the average value of the longitudinal momentum fraction in the fragmentation process increases, the correlation between the charge of the hadron and the charge of the fragmenting quark increases.

As a third example, the study using different beam types is considered. By taking appropriate combinations of the beam types one can isolate a sample of events dominated by the annihilation process. In such a sample the awayside charge ratio should be approximately equal to one, since the fragmenting parton is a gluon in this case. 
In this experiment one can actually measure the fragmentation and structure functions of the partons. In order to do this, the jet on the away-side must be reconstructed, thereby constraining the calculation of the parton scattering variables.

In this thesis, the experimental results from E706 will be compared with the above mentioned aspects. This will be done in Chapter 8 . Since the theory of the event structure is better understood at higher $p_{T}$, the data used in this thesis are from events in which the triggering particle has at least $4 \mathrm{GeV} / \mathrm{c} p_{T}$.

\subsection{E706 experimental program}

One of the problems in using direct photons as probes is the fact that the yield of direct photons is greatly reduced relative to the yield of jets. There are many more subprocesses which contribute to the production of hadron jets than to the yield of direct photons. Furthermore the QCD coupling constant $\alpha_{S}$ is an order of magnitude stronger than the fine structure constant $\alpha$. Consequently the $\gamma /$ jet ratio is expected $[29]$ to be small $(<0.001)$.

The main background to the direct photon production is the production of photons from the decays of $\pi^{0}$ 's and $\eta$ 's. This can be seen from the $\gamma \gamma$ mass plot in Figure 1.4. Extraction of the direct photon signal from the decays of these particles is technically challenging [29]. Fortunately, since the photon carries away the entire $p_{T}$ in the elementary collision, whereas the $\pi^{0}$ 's and $\eta$ 's are the fragments of the constituents (typically with small 
momentum fractions), it is expected that for a fixed angle in the center of mass the $\gamma /$ hadron ratio will increase with $p_{T}$ and the $\gamma$ yield will eventually surpass that of $\pi^{0}$ s. This has been verified by various experiments in the ISR at CERN [2]. In Figure 1.5 the cross section for $\pi^{0}$ 's as a function of $p_{T}$ is shown from the data from E706. Similariy, in Figure 1.6 the cross section for single photons is shown as a function of $p_{T}$ from the data from E706. The cross sections for both processes fall exponentially with increasing $p_{T}$ (about an order of magnitude for each $1 \mathrm{GeV} / \mathrm{c}$ in $p_{T}$ ). Figure 1.7 shows the ratio of $\gamma / \pi^{0}$ from this experiment. We observe that this ratio does indeed rise with increasing $p_{T}$.

By measuring the properties of inclusive $\pi^{0}$ and $\eta$ production one can then estimate the background from these decays and hence determine the true direct photon cross section statistically. For this reason E706 also determines the $\pi^{0}$ production properties. In this thesis, a comparison of the structure of events for $\gamma$ and $\pi^{0}$ production will be made.

\subsection{Variables and notations used}

In this section the mathematical expressions pertinent to the subsequent analysis are discussed. Here the upper case letters will be used to designate the hadrons and the lower case letters for the hadron constituents which undergo the hard scattering. The notations used are those displayed in Figure 1.3.

For the process $A+B \rightarrow C+D$, the $\mathrm{A}$ and $\mathrm{B}$ are initial state hadrons 


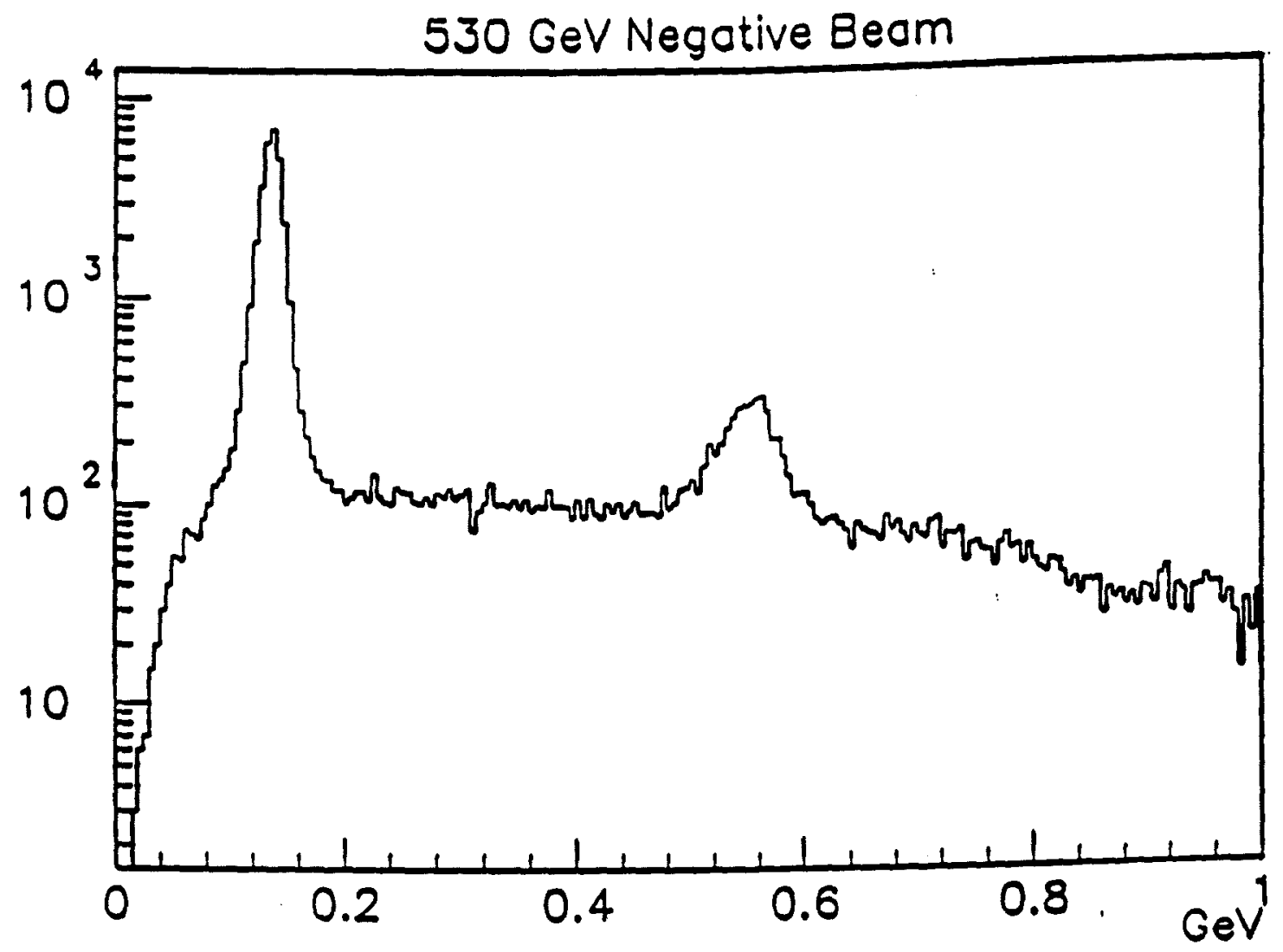

Figure 1.4: $\gamma \gamma$ mass plot 


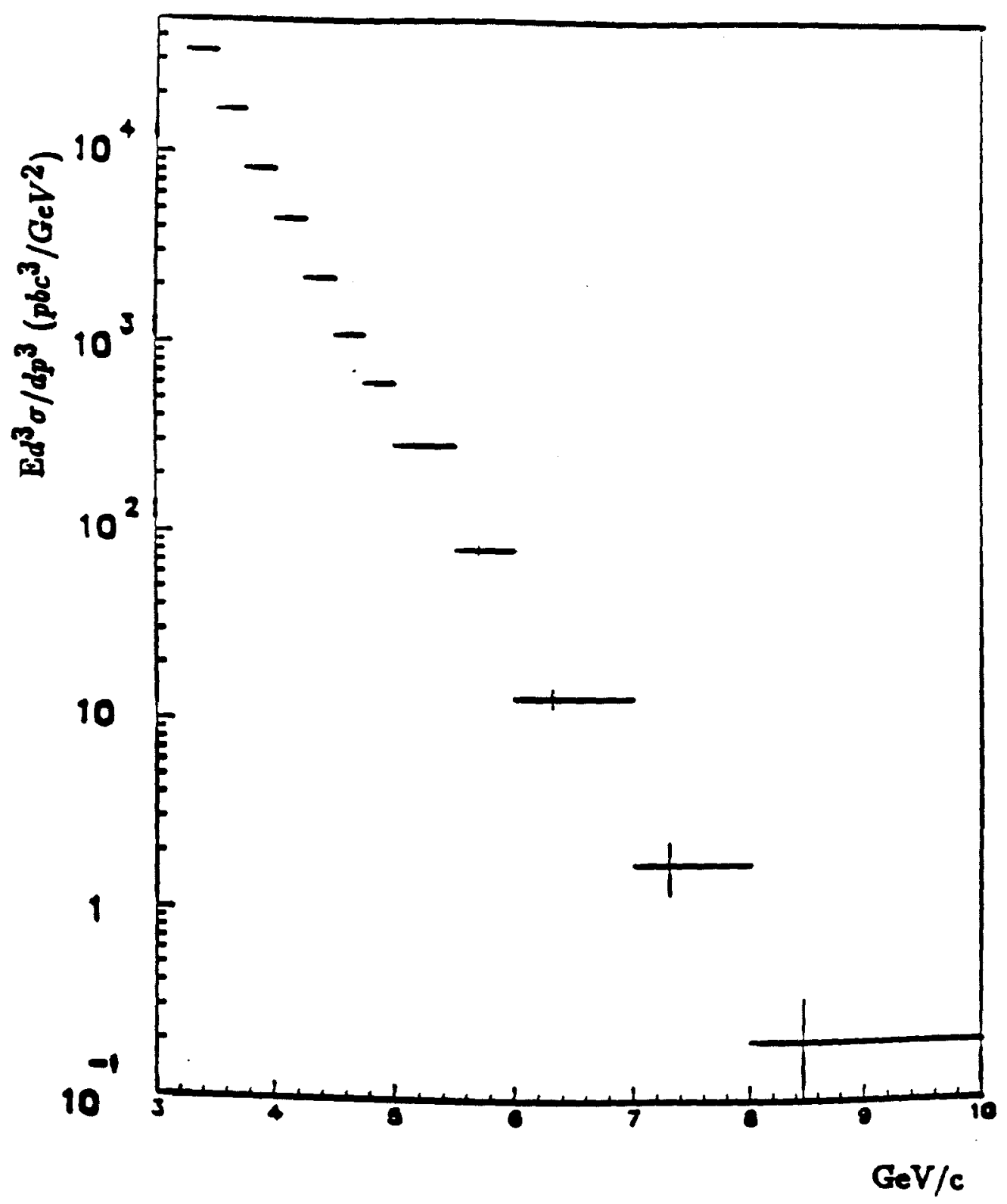

Figure 1.5: $d \sigma / d p_{T}$ for $\pi^{0}$ 's in the process $\pi^{-}+B e \rightarrow \pi^{0}+X$ 


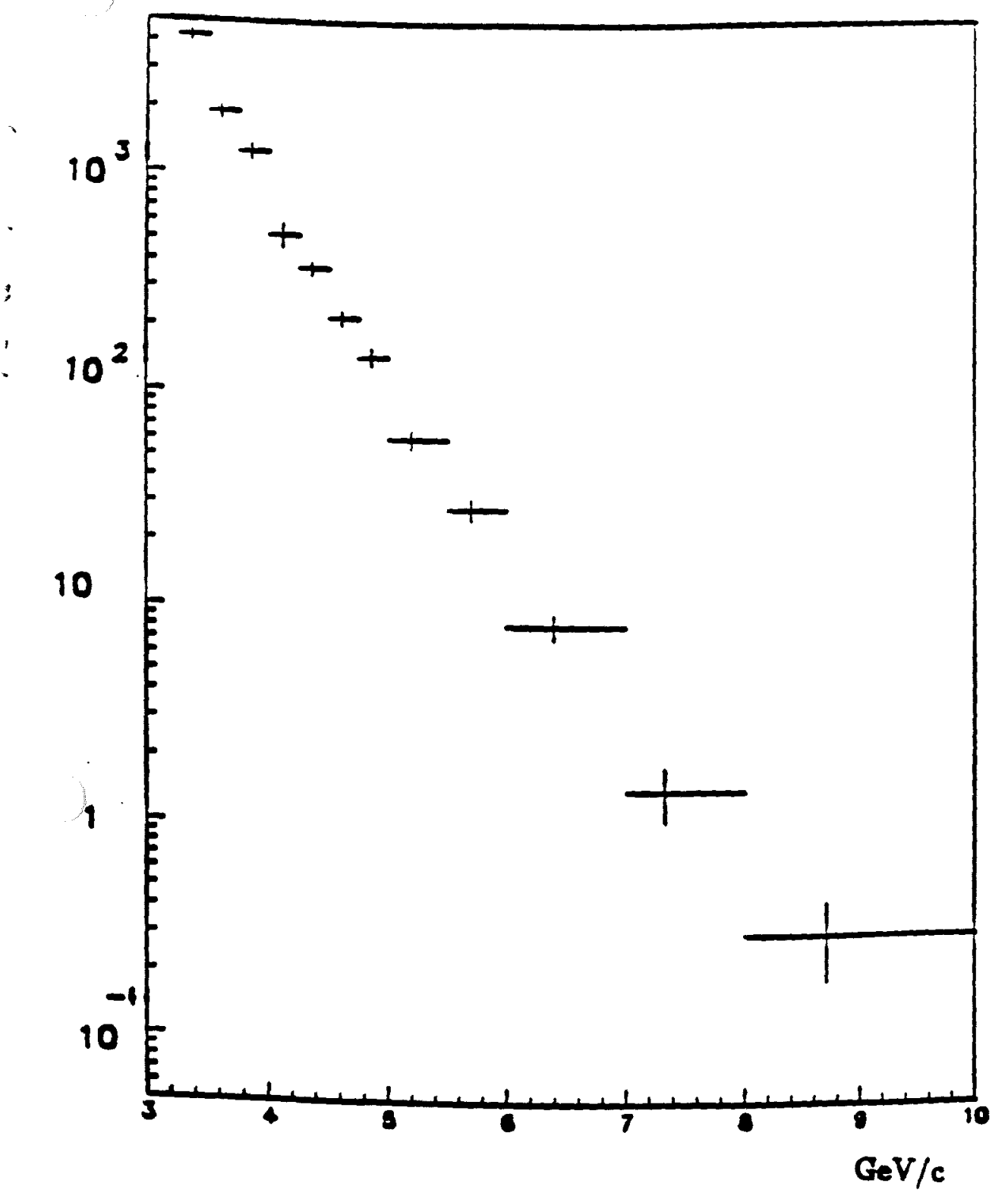

Figure 1.6: $d \sigma / d p_{T}$ for $\gamma^{\prime}$ 's in the process $\pi^{-}+B e \rightarrow \gamma+X$ 


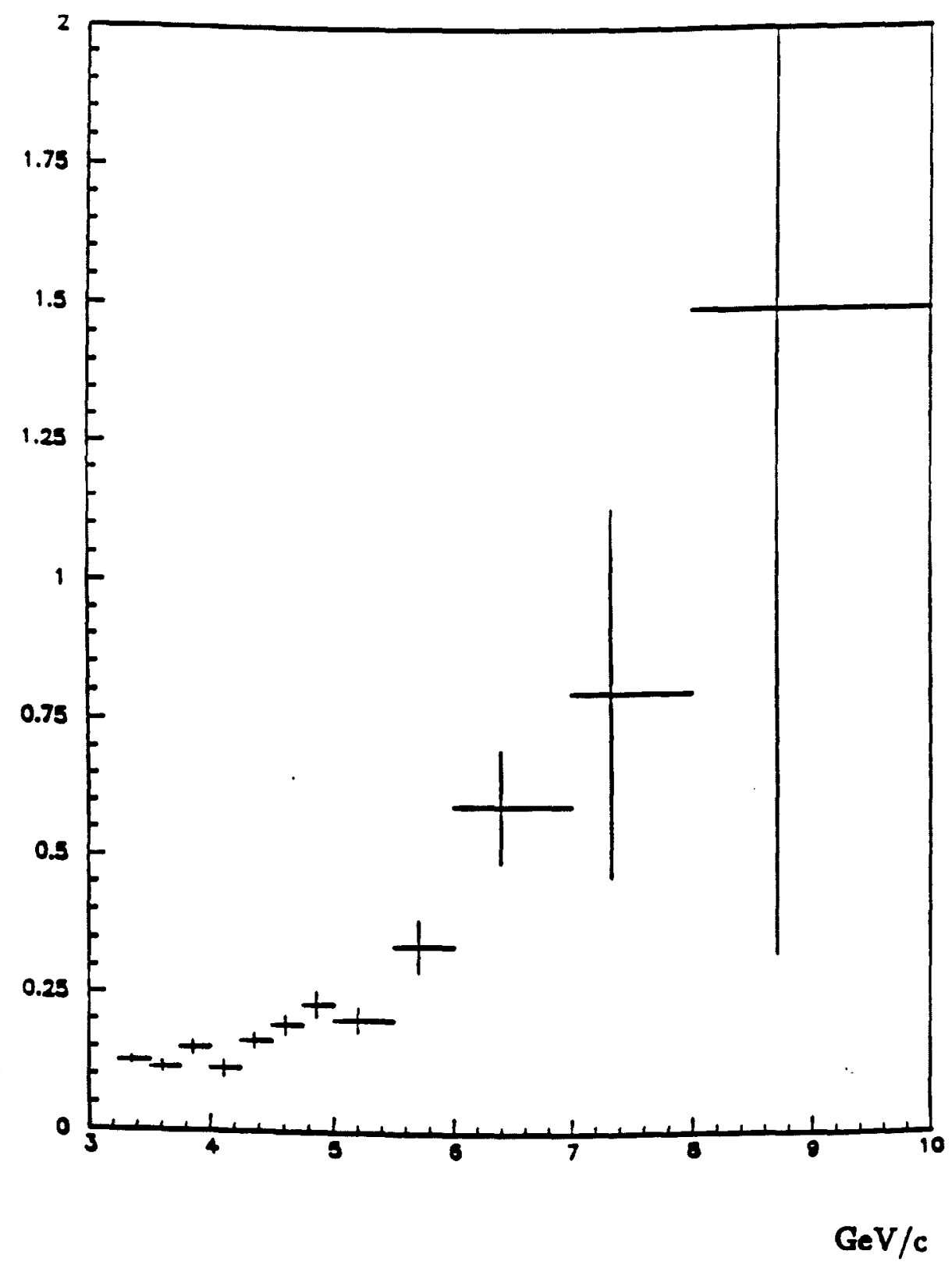

Figure 1.7: Ratio of $\gamma / \pi^{0}$ versus $p_{T}$ 
and $\mathrm{C}$ is the observed final state hadron with four-vectors $p_{A}, p_{B}$ and $p_{C}$, respectively. The Mandelstam variables are defined as:

$$
\begin{aligned}
& s=\left(p_{A}-p_{B}\right)^{2} \\
& t=\left(p_{A}-p_{C}\right)^{2} \\
& u=\left(p_{B}-p_{C}\right)^{2}
\end{aligned}
$$

Here $s$ is the square of the total center of mass energy and $t$ and $u$ are the squares of the momentum transfers from particles $\mathrm{A}$ and $\mathrm{B}$ to particle $\mathrm{C}$, respectively.

A similar set of variables exists for the constituent scattering subprocess $a+b \rightarrow c+d$, and a symbol " $"$ will be placed over the corresponding variables. For the case of a massless two body scattering the Mandelstam variables satisfy the constraint $\hat{s}+\hat{t}+\hat{u}=0$. For direct photon production, the direct photon is same as particle $\mathrm{C}$ which is same as parton c.

Let:

$p_{T}=$ momentum component of a particle normal to the beam direction ${ }^{p} L=$ momentum component of a particle parallel to the beam direction $E=$ energy of the particle and

$$
\begin{aligned}
& { }^{x_{T}}=\frac{2 p_{T}}{\sqrt{s}} \\
& x_{F}=\frac{2 p_{L}}{\sqrt{s}}
\end{aligned}
$$

With these definitions, the allowed ranges of $x_{T}$ and $x_{F}$ are $(0,1)$ and $(-1,1)$, respectively, if the masses of the hadrons are neglected. 
The rapidity of a particle is defined as:

$$
y=0.5 \ln \frac{E+p_{L}}{E-p_{L}}
$$

Let parton $a$ carry a fraction $x_{a}$ of hadron A's longitudinal momentum and let parton $b$ carry a fraction $x_{b}$ of hadron B's longitudinal momentum. Then in the overall hadron-hadron center-of-mass system the 4-vectors for $a$ and $b$ can be written (assuming massless partons and neglecting any parton transverse momenta) as:

$$
\begin{gathered}
p_{a}=x_{a} \frac{\sqrt{s}}{2}(1,0.0 .1) \\
p_{b}=x_{b} \frac{\sqrt{s}}{2}(1,0,0,-1)
\end{gathered}
$$

where the positive $z$-axis is taken to be along the direction of the incident hadron $\mathrm{A}$.

If the scattered parton $c$ has a transverse momentum $p_{T}$ and rapidity $y_{1}$, then:

$$
p_{c}=p_{T}\left(\cosh \left(y_{1}\right), 1,0, \sinh \left(y_{1}\right)\right)
$$

If the scattered parton $\mathrm{d}$ has a rapidity $y_{2}$, then:

$$
p_{d}=p_{T}\left(\cosh \left(y_{2}\right), 1,0, \sinh \left(y_{2}\right)\right)
$$

(since $\mathrm{c}$ and $\mathrm{d}$ have the same $p_{T}$ ). Hence:

$$
\hat{s}=x_{a} x_{b} s=M^{2}
$$

where $M$ is the effective invariant mass of the 2 -jet system, and

$$
\hat{t}=-x_{a} p_{T} \sqrt{s} e^{-y_{1}}=-x_{b} p_{T} \sqrt{s} e^{y_{2}}
$$




$$
\hat{u}=-x_{b} p_{T} \sqrt{s} e^{y_{1}}=-x_{a} p_{T} \sqrt{s} e^{-y_{2}}
$$

Also:

$$
\begin{gathered}
x_{a}=\frac{p_{T}}{\sqrt{s}}\left(e^{y_{1}}+e^{y_{2}}\right) \\
x_{b}=\frac{p_{T}}{\sqrt{s}}\left(e^{\left.-y_{1}+e^{-y_{2}}\right)}\right.
\end{gathered}
$$

\subsubsection{Cross sections}

In the absence of parton $k_{T}$ effects (i.e. that the partons do not have a momentum component normal to the hadron's momentum vector) the dijet cross section can be written in terms of the rapidities of the trigger and recoil jets and their transverse momentum $p_{T}$.

To the lowest order in the running coupling constant, for the two subprocesses, namely, Compton scattering and annihilation process, the differential cross sections after performing color sums and spin averages [25] can be written as:

$$
\begin{aligned}
& \frac{d \sigma}{d y_{1} d y_{2} d p_{T}^{2}}\left(A B \rightarrow j e t_{1}+j e t_{2}+X\right)= \\
& \sum_{a b} \int d x_{a} d x_{b} G_{a / A}\left(x_{a}\right) G_{b / B}\left(x_{b}\right) \frac{\hat{s}}{2} \frac{d \sigma}{d \hat{t}}(a b \rightarrow 12) \\
& \delta\left(x_{a} \frac{\sqrt{s}}{2}+x_{b} \frac{\sqrt{s}}{2}-p_{T} \cosh y_{1}-p_{T} \cosh y_{2}\right) \\
& \delta\left(x_{a} \frac{\sqrt{s}}{2}-x_{b} \frac{\sqrt{s}}{2}-p_{T} \sinh y_{1}-p_{T} \sinh y_{2}\right)
\end{aligned}
$$

Here the $\delta$ functions are to ensure that the jets are produced with equal and opposite transverse momenta. By integrating equation 1.16 , the resultant 
2 -jet cross section is:

$\frac{d \sigma}{d y_{1} d y_{2} d p_{T}^{2}}\left(A B \rightarrow j e t_{1}+j e t_{2}+X\right)=\sum_{a b} x_{a} G_{a / A}\left(x_{a}\right) x_{b} G_{b / B}\left(x_{b}\right) \frac{d \sigma}{d \hat{t}}(a b \rightarrow 12)$

Hence. from the knowledge of the four-vectors of the two jets the kinematics of the parton scattering process can be completely determined.

The objective of experiment E706 is to determine the structure functions and fragmentation functions. Here one of the resultant jets is the direct photon or $\pi^{0}$. For the case of a Bremsstrahlung photon, the appropriate photon fragmentation function has to be used. Figure 1.8 shows the DukeOwens structure functions [25]. We note that E706 is particularly sensitive to the range $0.25<x_{a}, x_{b}<0.5$.

\subsection{Outline of the thesis}

In the following chapters a description of the E706 apparatus is presented. This is followed by a description of the construction of the Multiwire proportional chambers. This in turn is followed by a description of how the data were acquired. The remaining chapters are dedicated to a description of the reconstruction and analysis of the data. 


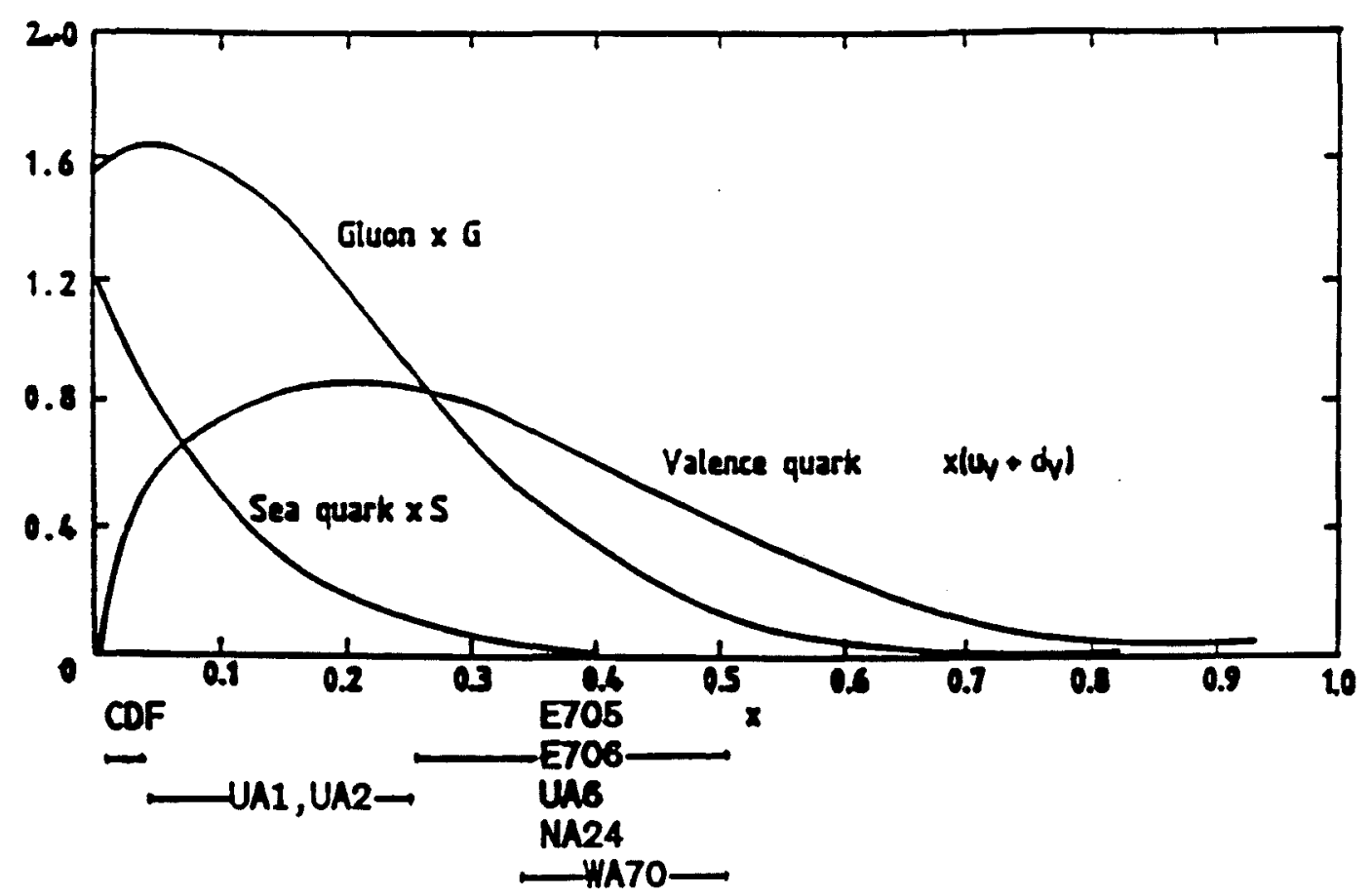

Figure 1.8: Plot of structure functions 


\section{Chapter 2}

\section{Experimental Setup}

\subsection{Overall layout}

Figure 2.1 shows the overall layout of the spectrometer. It consists of silicon strip detectors (SSD), a set of multiwire proportional chambers (MWPC), electromagnetic (EM) and hadronic calorimeters, a forward calorimeter and a set of trigger scintillators.

The coordinate system is defined as follows (Figure 2.2 ). The $Z$ axis is along the nominal beam direction. The $\mathrm{X}$ axis points to the left while looking downstream along the beam. The $\mathrm{Y}$ axis is pointed upwards. The origin is located at a point $9 \mathrm{~m}$ upstream of the front face of the electromagnetic calorimeter. The $\phi=0$ axis is along the positive $\mathrm{X}$ direction. The $\theta=0$ axis is along the positive $\mathrm{Z}$ direction.

\subsection{Beam line and veto walls}

The primary beam from the accelerator is incident on a target at the beginning of the secondary beam line. During one part of the running period 


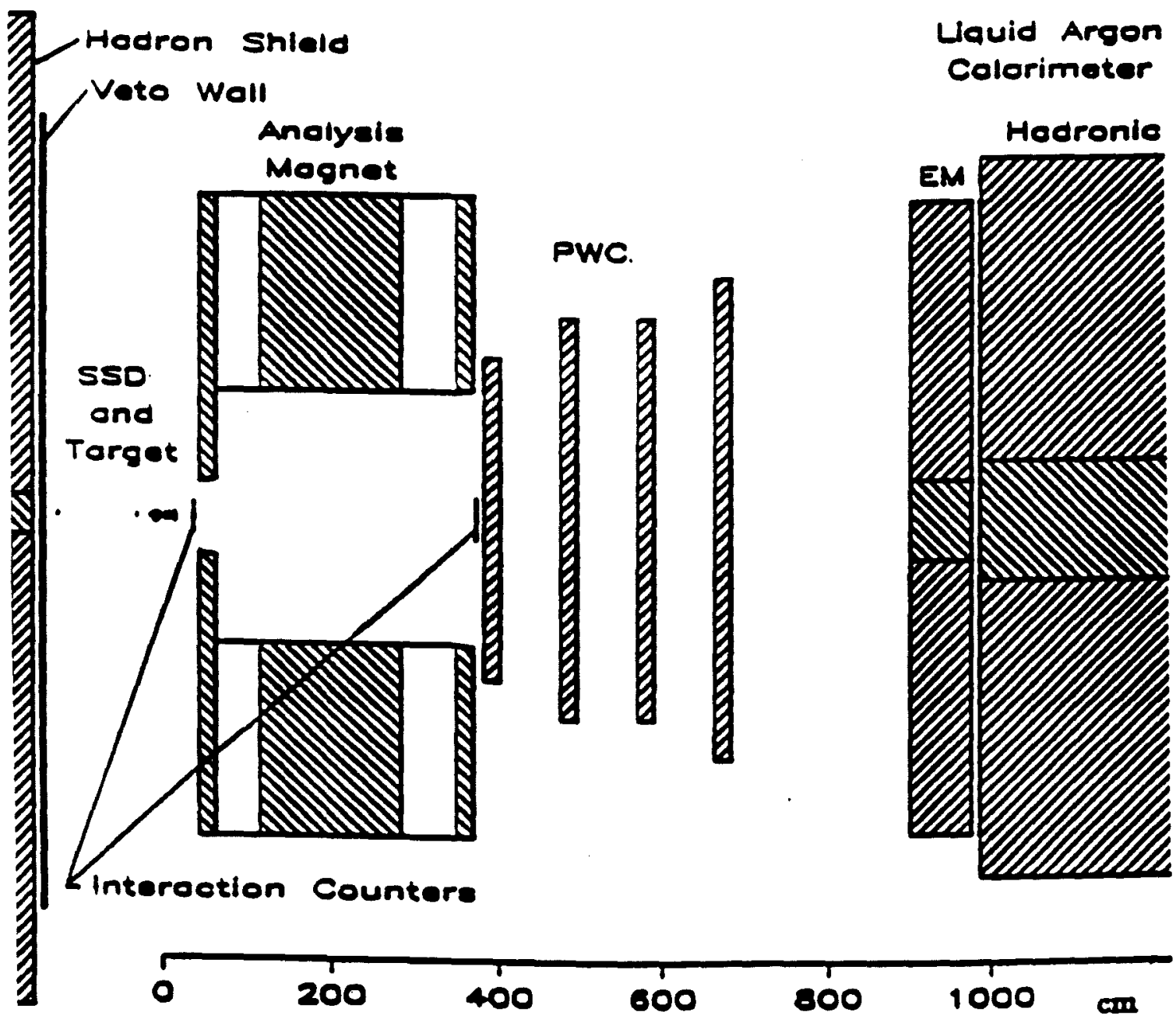

Figure 2.1: Picture of MWEST 

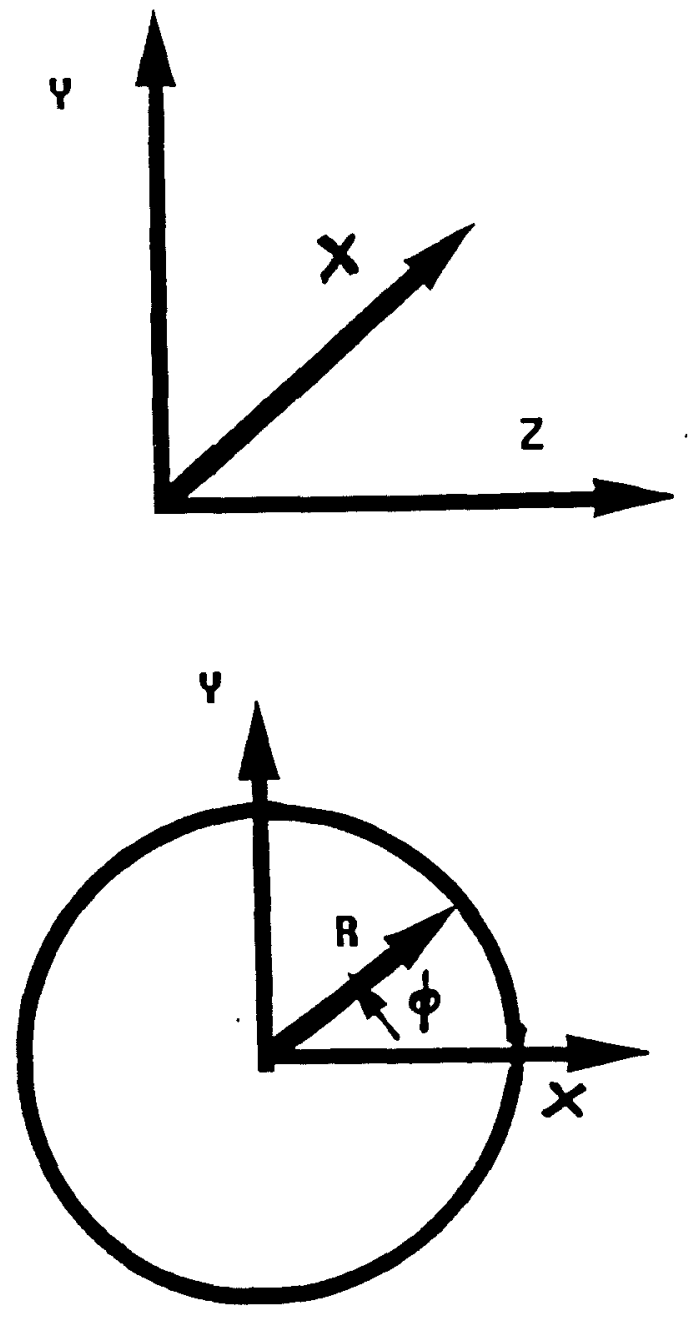

Figure 2.2: E706 coordinate system 
the secondary beam line carried a $\pi^{-}$beam and during the other part it carried $\pi^{-}$and proton beams. A differential Cerenkov counter in the beam line distinguished the $\pi^{\top} / \mathrm{p}$ beam particles. The momentum of the beam was about $530 \mathrm{GeV} / \mathrm{c}$.

The particles which are produced during the transport of the beam through the beam line tend to form a halo around the beam pipe. These particles are mainly muons from $\pi$ and $\mathrm{K}$ decays. Shielding blocks are kept around the beam line upstream of the target to absorb these muons. In addition, two planes of scintillators are kept around the beam. upstream of the target. A halo particle would produce a coincidence signal in these scintillators. These scintillators are called the veto walls. They are made in 4 parts, each covering one quarter of the region around the beam.

\subsection{Target}

Various segmented targets were used at different times of the data taking. The advantage of using a segmented target is in distinguishing the secondary interactions as well as decay particle vertices. The secondary interactions are more likely to occur in the solid target. The air gap between the target segments will only have decay vertices.

An isoscalar target was used for the most part of the run. The reason for this was that, the cross section for $\pi^{0}$ production from an isoscalar target is the same for the cases when either a $\pi^{+}$or $\pi^{-}$beam particle hits the target. Since $\pi^{0}$ s are the main source of background to the direct photon 
Table 2.1: Types and characteristics of targets

\begin{tabular}{|l|c|c|c|}
\hline Target type & Beryllium & Copper & Carbon \\
\hline x width $(\mathrm{cm})$ & 2.0 & 2.0 & 2.0 \\
y width $(\mathrm{cm})$ & 2.0 & 2.0 & 2.0 \\
Number of segments & 20 & 2 & 20 \\
Segment thickness (cm) & 0.16 & 0.16 & 0.16 \\
Gap thickness (cm) & 0.2 & 0.2 & 0.2 \\
Total interaction length & 0.1 & 0.01 & 0.1 \\
Total radiation length & 0.1 & 0.1 & 0.2 \\
\hline
\end{tabular}

production, an isoscalar target helps one to achieve the similar backgrounds.

The different materials allow a study of the A dependence of direct photon production [33]. Table 2.1 gives the types of targets used, their sizes and their interaction and radiation lengths.

\subsection{Charged particle spectrometer}

The charged particle spectrometer consists of a Silicon Strip Detector (SSD) system, a dipole magnet and a set of Multiwire Proportional Chambers (MWPC).

A set of SSD's is located downstream of the target. They register the location of the charged tracks at various planes transverse to the beam direction. This information is used to reconstruct the charged tracks upstream of the magnet. These tracks are used to reconstruct the location of 
the primary vertex in the event. There is a set of SSD's upstream of the target as well. They are used to reconstruct the location and direction of the beam.

The MWPC's are located downstream of the magnet. They also register the location of the charged tracks at various planes transverse to the beam direction. This information is used to reconstruct the trajectories of the tracks which go through them.

The dipole magnet is used to bend the charged tracks in the horizontal plane (XZ) plane. By measuring the amount of bending, the charge and momentum of the tracks are measured. The amount of bending of the tracks is estimated using the tracks reconstructed in both the SSD's and the MIWPC's.

The resolution and efficiency of the charged particle spectrometer are described in Chapter 5.

\subsubsection{Silicon strip detector}

\section{General description of the system}

The Silicon Strip Detector (SSD) [18] system contains seven modules of silicon strip wafers. Four of the modules have wafers of size $3 \mathrm{~cm}$ by $3 \mathrm{~cm}$. The other three modules have wafers of size $5 \mathrm{~cm}$ by $5 \mathrm{~cm}$. All the wafers have pitch (strip width) of $50 \mu \mathrm{m}$. Each module consists of two planes. Each plane has equally spaced silicon strips. In the first plane the strips are oriented in the vertical direction and this plane is called the X-plane. In 
the second plane the strips are oriented in the horizontal direction and this plane is called the Y-plane. Figure 2.3 shows a diagram of the SSD system.

A minimum ionizing particle traversing one of the modules produces $(2.0-2.4) \cdot 10^{4}$ electron-hole pairs, depending upon the thickness of the wafer. The charge collection time is about $20 \mathrm{~ns}$. The collected charge is fed into a charge sensitive preamplifier. The output of these preamplifiers is further amplified, then discriminated and converted to logic signals for storage as digital data.

Three of the small modules, each consisting of an $X$ and $Y$ plane, are located upstream of the target. Their function is to measure the direction and position of the beam particles; they are called the beam SSD's. The four remaining modules are downstream of the target; because of the main function they provide, they are called the vertex SSD's. Figure 2.4 shows a typical event as seen in the SSD's. Table 2.2 shows the active area of each of the SSD planes. The total number of SSD channels to be readout is 7200 .

\section{Multiple scattering and hot channels}

Since the tracks produced at a point in the target have to go through the remaining target material and through the material of the SSD's $(0.3 \%$ interaction length), they will be subjected to multiple scattering and secondary interactions. The tracks which are produced by this process will complicate the subsequent charged particle reconstruction.

The hot channels are the strips of the SSD's which appear to register 


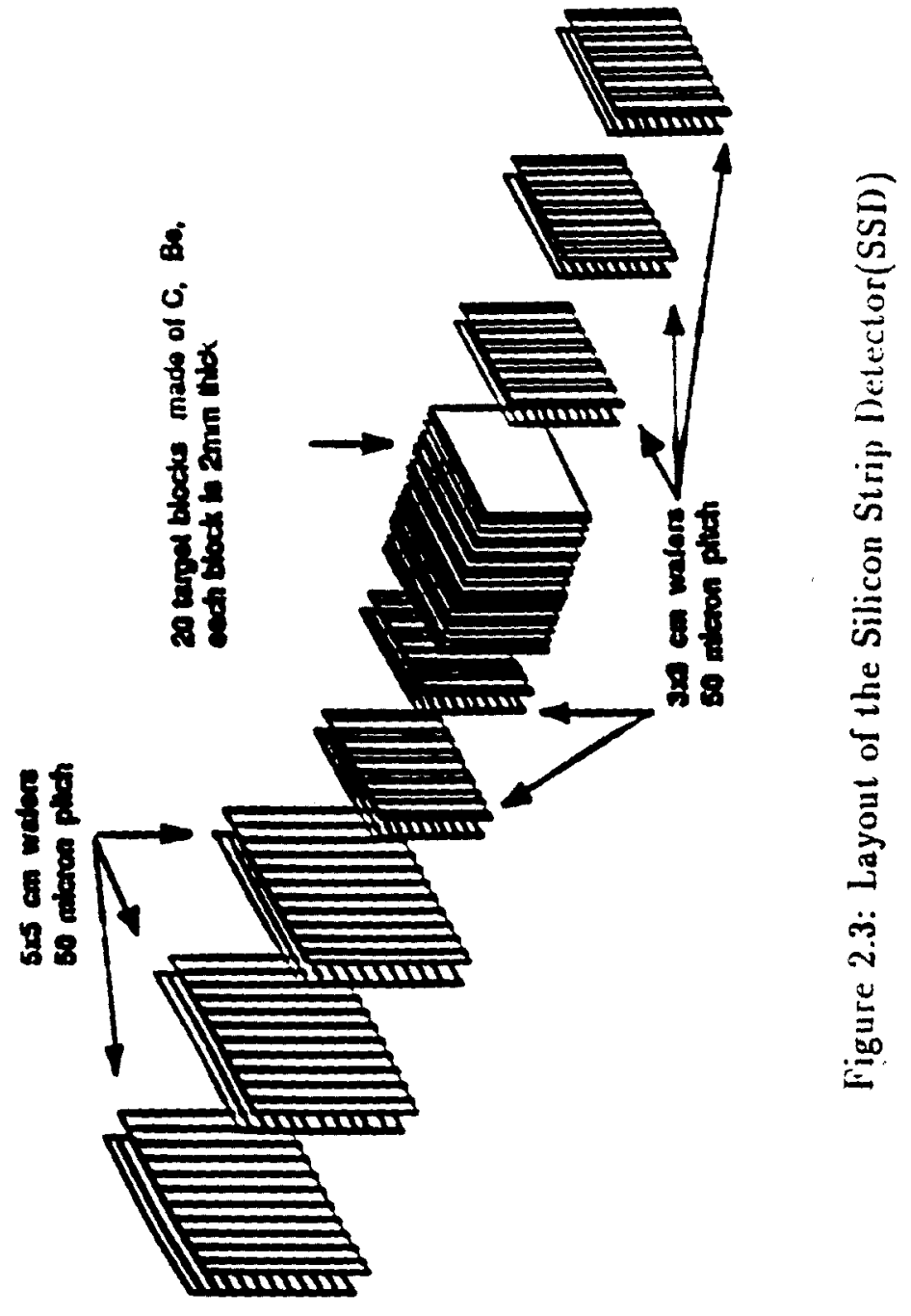




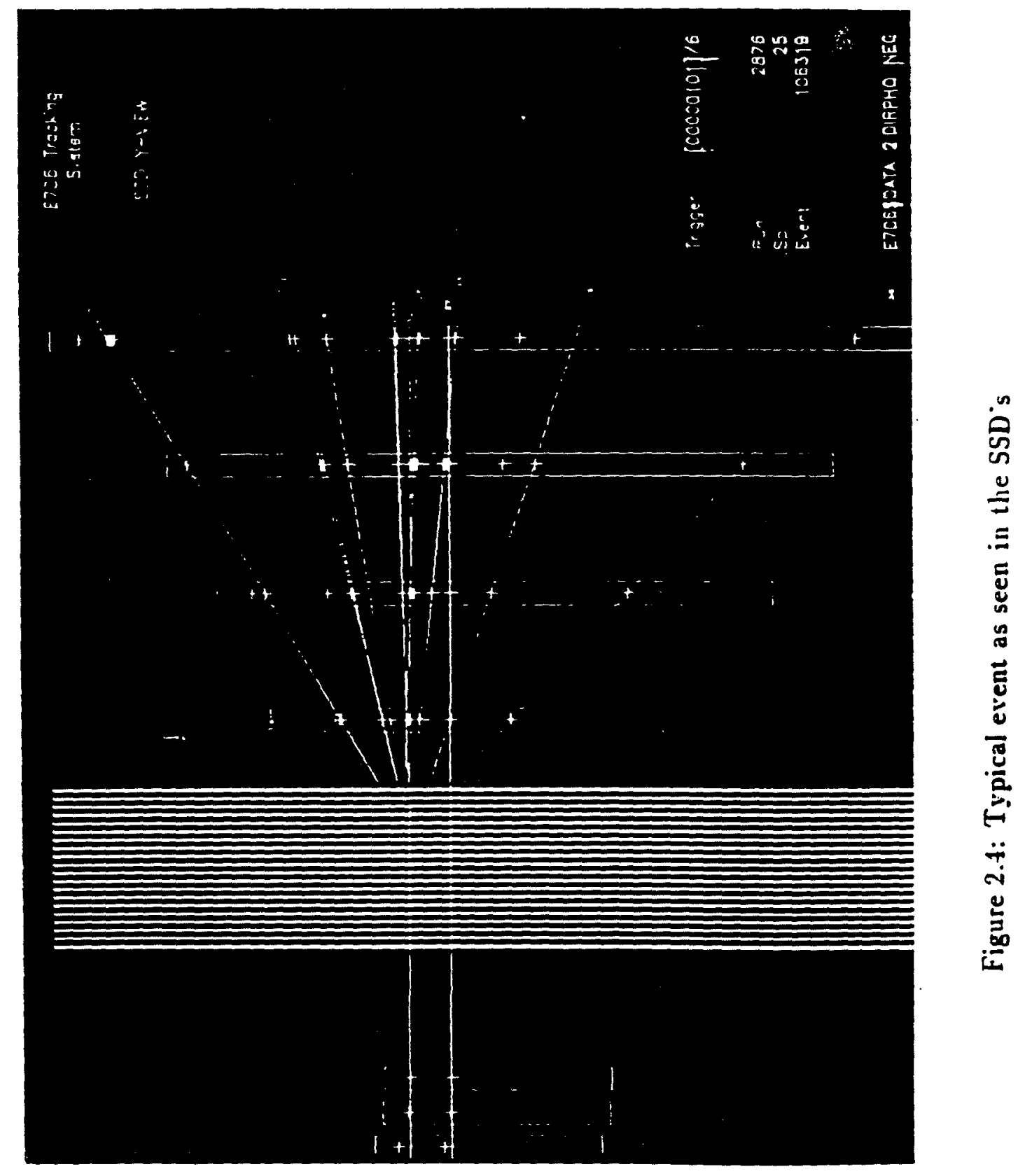


Table 2.2: Properties of the SSD's

\begin{tabular}{|c|c|c|c|c|}
\hline SSD plane & $\begin{array}{l}X \text { width } \\
(\mathrm{cm})\end{array}$ & $\begin{array}{l}Y^{r} \text { width } \\
(\mathrm{cm})\end{array}$ & $\begin{array}{c}\text { Z thickness } \\
(\mathrm{cm})\end{array}$ & $\begin{array}{c}\text { Z location } \\
(\mathrm{cm})\end{array}$ \\
\hline 1 & 1.5 & 1.5 & 0.15 & -132.51 \\
\hline 2 & 1.5 & 1.5 & 0.15 & -132.52 \\
\hline 3 & 1.5 & 1.5 & 0.15 & -36.4 \\
\hline 4 & 1.5 & 1.5 & 0.15 & -36.4 \\
\hline 5 & 1.5 & 1.5 & 0.15 & -18.9 \\
\hline 6 & 1.5 & 1.5 & 0.15 & -18.9 \\
\hline 7 & 1.5 & 1.5 & 0.15 & -1.8 \\
\hline 8 & 1.5 & 1.5 & 0.15 & -1.8 \\
\hline 9 & 2.5 & 2.5 & 0.15 & 4.5 \\
\hline 10 & 2.5 & 2.5 & 0.15 & 4.5 \\
\hline 11 & 2.5 & 2.5 & 0.15 & 9.25 \\
\hline 12 & 2.5 & 2.5 & 0.15 & 9.25 \\
\hline 13 & 2.5 & 2.5 & 0.15 & 14.7 \\
\hline 14 & 2.5 & 2.5 & 0.15 & 14.7 \\
\hline
\end{tabular}


a signal all the time. They can be either due to noisy electronic channels or due to very high leakage current in the SSD. They have to be properly accounted for while performing the reconstruction of the tracks.

\subsubsection{Multiwire proportional chamber system}

Only a brief description of the MWPC system will be given here. Additional details are given in Chapter 3 and Appendix B. The MWPC system is located downstream of the magnet, between $\mathrm{Z}=360 \mathrm{~cm}$ and $\mathrm{Z}=700 \mathrm{~cm}$, as shown in Figure 2.1. and consists of four chambers. Each of the chambers has four planes of wires called "anode planes." The wires in an anode plane are equally spaced and the orientations of the wires in each of the four anode planes are $0^{\circ}, 90^{\circ}, 36.87^{\circ}$ and $-53.13^{\circ}$ with respect to the vertical (Y) direction in the E706 coordinate system. These planes are labelled X, $\mathrm{Y}, \mathrm{U}$ and $\mathrm{V}$ planes, respectively.

Each anode plane is sandwiched between two cathode planes which are made of mylar with a thin coating of graphite. The cathode is kept at a large negative potential and the anode plane is grounded. Table 2.3 shows the sizes and other relevant dimensions of the various parts of the MWPC system. Figure 2.5 shows a diagram of the various planes in the MWPC system and Figure 2.6 shows a photograph of the completed chambers. The total number of MWPC channels is 14000 . 
Table 2.3: Dimensions of MWPC

\begin{tabular}{|c|c|c|c|c|c|c|c||}
\hline Plane & Plane & Angle & X width & Y width & No. of & Spacing & $\mathrm{Z}$ \\
no. & label & & $(\mathrm{cm})$ & $(\mathrm{cm})$ & wires & $(\mathrm{cm})$ & $(\mathrm{cm})$ \\
\hline 1 & $\mathrm{X}$ & $90.00^{\circ}$ & 162.56 & 121.92 & 640 & 0.254 & 380.2 \\
2 & $\mathrm{Y}$ & $0.00^{\circ}$ & 162.56 & 121.92 & 480 & 0.254 & 382.0 \\
3 & $\mathrm{U}$ & $-53.13^{\circ}$ & 162.56 & 121.92 & 704 & 0.254 & 383.7 \\
4 & $\mathrm{~V}$ & $36.87^{\circ}$ & 162.56 & 121.92 & 672 & 0.254 & 385.4 \\
5 & $\mathrm{X}$ & $90.00^{\circ}$ & 203.2 & 207.91 & 800 & 0.254 & 471.1 \\
6 & $\mathrm{Y}$ & $0.00^{\circ}$ & 203.2 & 207.91 & 800 & 0.254 & 474.9 \\
7 & $\mathrm{U}$ & $-53.13^{\circ}$ & 203.2 & 207.91 & 896 & 0.254 & 476.6 \\
8 & $\mathrm{~V}$ & $36.87^{\circ}$ & 203.2 & 207.91 & 896 & 0.254 & 478.4 \\
9 & $\mathrm{X}$ & $90.00^{\circ}$ & 203.2 & 207.91 & 800 & 0.254 & 567.5 \\
10 & $\mathrm{Y}$ & $0.00^{\circ}$ & 203.2 & 207.91 & 800 & 0.254 & 569.3 \\
11 & $\mathrm{U}$ & $-53.13^{\circ}$ & 203.2 & 207.91 & 896 & 0.254 & 571.0 \\
12 & $\mathrm{~V}$ & $36.87^{\circ}$ & 203.2 & 207.91 & 896 & 0.254 & 572.7 \\
13 & $\mathrm{X}$ & $90.00^{\circ}$ & 243.58 & 243.84 & 960 & 0.254 & 660.5 \\
14 & $\mathrm{Y}$ & $0.00^{\circ}$ & 243.58 & 243.84 & 960 & 0.254 & 662.3 \\
15 & $\mathrm{U}$ & $-53.13^{\circ}$ & 243.58 & 243.84 & 1120 & 0.254 & 664.1 \\
16 & $\mathrm{~V}$ & $36.87^{\circ}$ & 243.58 & 243.84 & 1120 & 0.254 & 665.8 \\
\hline
\end{tabular}




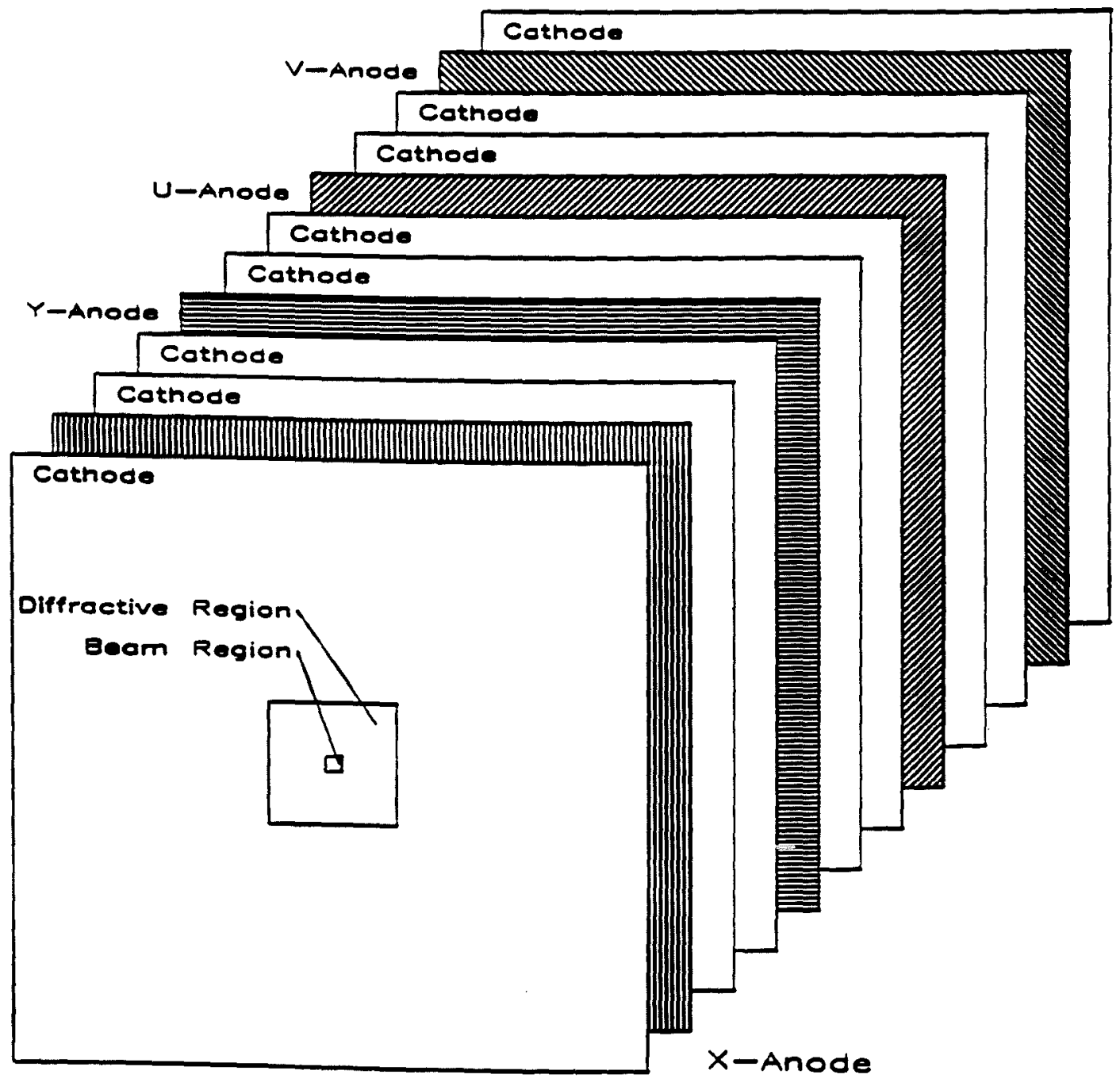

Figure 2.5: Planes of MWPC 


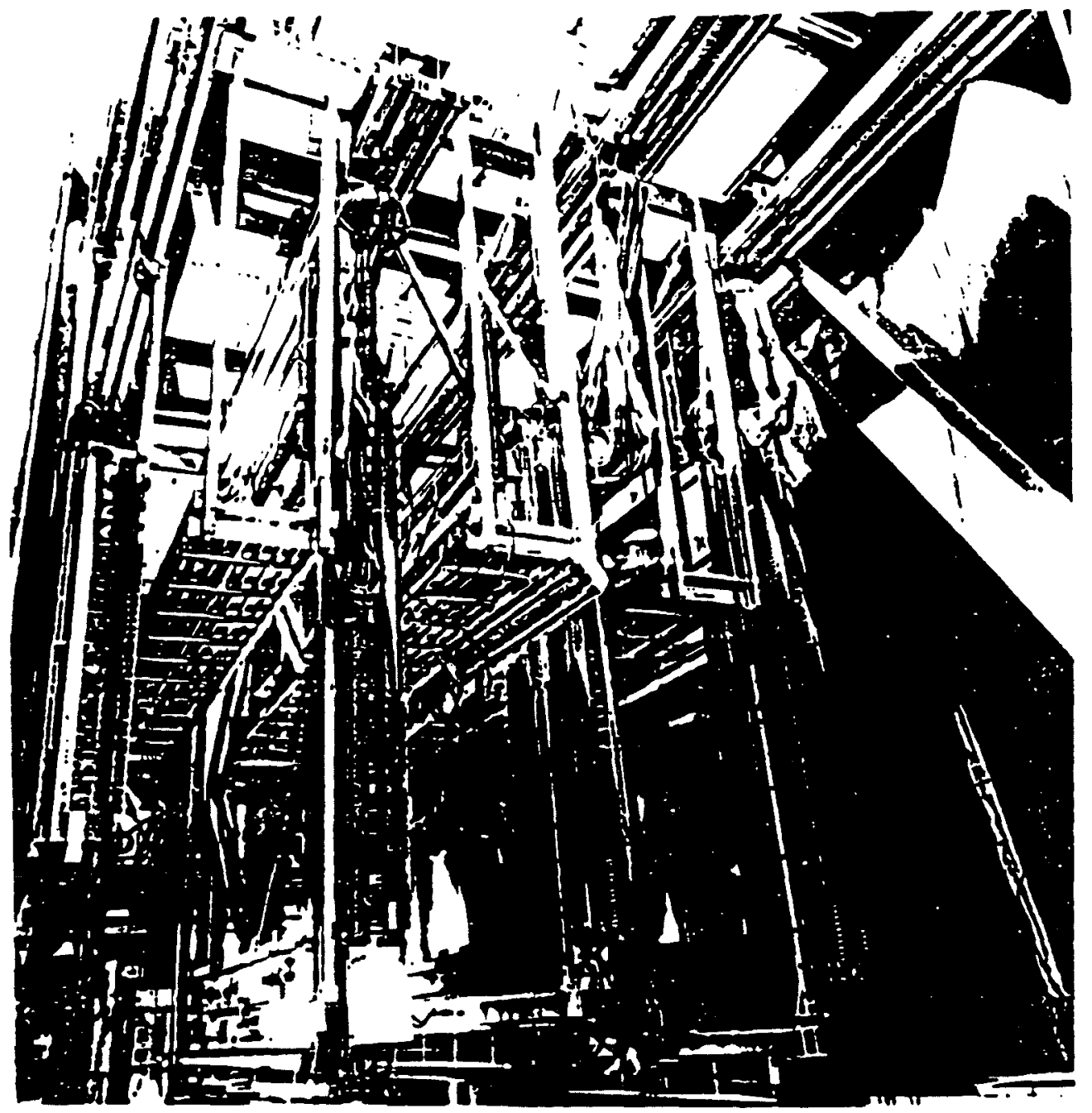

Figure 2.6: Picture of completed chambers 


\subsection{Magnet}

\subsubsection{Characteristics}

The magnet has a length of $328 \mathrm{~cm}$ along the beam line. The size of its aperture increases from the upstream end to the downstream end. Large steel plates are located at the upstream and downstream ends of the magnet in order to absorb the fringe fields. They are called the mirror plates. The upstream mirror plate has a rectangular opening of size $17.8 \mathrm{~cm}$ by $12.7 \mathrm{~cm}$ and the downstream mirror plate has a hole of size $68.6 \mathrm{~cm}$ by $62.4 \mathrm{~cm}$.

The direction of the field was in the positive or negative $Y$ direction, depending upon the direction of the current. These directions were switched at various times during the run. Further deatils of the magnetic field parameterization are presented in Appendix A.

\subsection{Liquid argon calorimeter}

\subsubsection{General description}

The Liquid Argon Calorimeter ( $\mathrm{LAC})(16,20,21]$ is a device to measure the energy and position of high energy particles and consists of an electromagnetic calorimeter (EMLAC) and a hadronic calorimeter (HALAC). Both of these are sampling calorimeters and therefore have layers of inactive energyabsorbing material interleaved with an active sampling medium. The active medium used here is liquid argon. For energy absorption, the EMLAC has lead sheets and the HALAC has steel plates. The use of liquid argon neces- 
sitates maintaining a complicated cryogenic environment for this detector 17]. In this detector both the electromagnetic and the hadron calorimeter are contained in a single dewar. Figure 2.7 shows a picture of the LAC.

\subsubsection{The electromagnetic calorimeter (EMLAC)}

\section{Geometry of the electromagnetic calorimeter}

The electromagnetic calorimeter (EMLAC) consists of four quadrants of a cylinder centered around the beamline. This is illustrated in Figure 2.8. The diameter of the cylinder is about $3 \mathrm{~m}$ and its thickness is about $0.75 \mathrm{~m}$ along the beam line. Each quadrant contains a core of alternating. lead absorber plates and copper clad G-10 (a type of glass epoxy laminate) boards. These G-10 boards are called anode planes. They contain the charge collection strips. At the upstream and downstream ends of this core there are additional readout boards, which map the detector strips to the signal cables. Each stack of these planes is enclosed in a structural frame that allows the four quadrants to be aligned and assembled into the completed calorimeter. The calorimeter has a hole in the center so that the non-interacting beam particles are prevented from entering the liquid argon. When assembled, the calorimeter has an internal radius of $21.1 \mathrm{~cm}$ (beam hole) and an external radius of $165.3 \mathrm{~cm}$. It is hung from a movable gantry enclosed in a cryogenic dewar, Figure 2.7.

The EMLAC lead plates are $2 \mathrm{~mm}$ thick and have the shape of a single quadrant. In order to provide mechanical stiffness a calcium-lead alloy was 


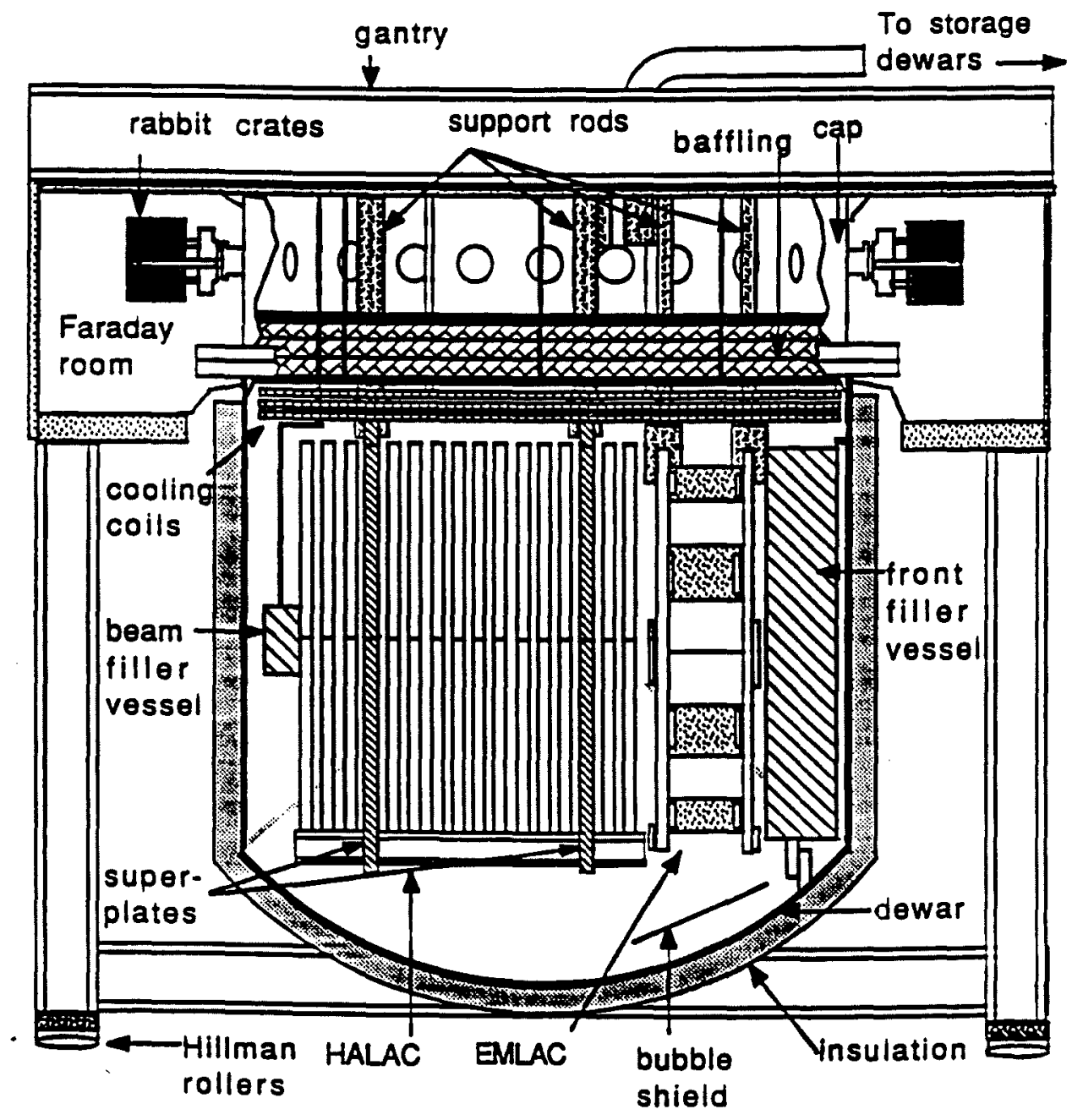

Figure 2.7: The liquid argon calorimeter 


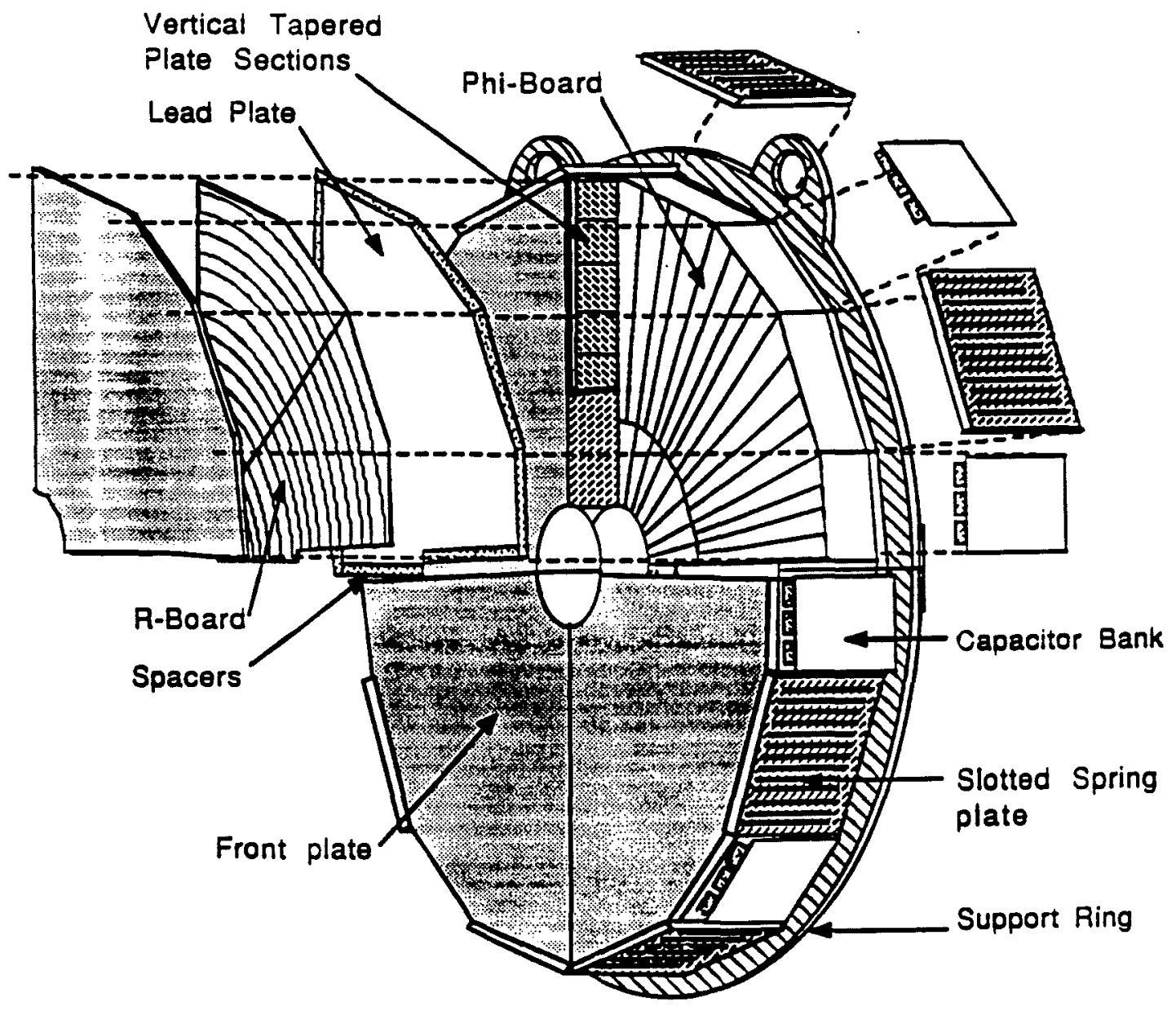

Figure 2.8: Electromagnetic calorimeter: $\mathrm{r}$ and and $\phi$ strips 
used. The calcium has a very low diffusivity at room temperature. These plates have $20 \%$ more electrical conductivity than those of pure lead.

For readout purposes the EMLAC can be considered as divided into four quadrants, each quadrant consisting of two octants. as seen in Figure 2.8. Along the axis of the cylinder EMLAC is divided into two sections called the "front" and "back" sections. There are 22 plates and boards in the front section and 44 plates and boards in the back section.

There are two alternating types of anode boards used in this calorimeter (Figure 2.8). One has strips which define the radial position (r-boards) of an electromagnetic shower and the other has strips which define the azimuthal angular position ( $\phi$-boards). Both types of boards have matching sets of strips on opposite sides of a board.

The r-strips are a series of concentric circular strips centered on the beamline. The width and location of these strips are determined according to their distance along the beam line from the target, the radial position of the center of the strip and the width of the innermost strip on the upstreammost "anode board" with r-strips. As a result, each of the r-strips subtends approximately the same solid angle and hence is focussed towards the target (Figure 2.9). There are 255 r-strips in each octant. The amount of energy measured in the r-strips gives a direct estimate of the transverse momentum inherent in a shower.

The $\phi$-strips are radial (sector) strips. They are of two types. The first type is called the "inner $\phi . "$ They run from the inner radius $(20 \mathrm{~cm})$ of the anode board to a radius of $40 \mathrm{~cm}$. There are 96 inner $\phi$ strips per quadrant 
$\Varangle B$ determines the strip width on all of the r-boards

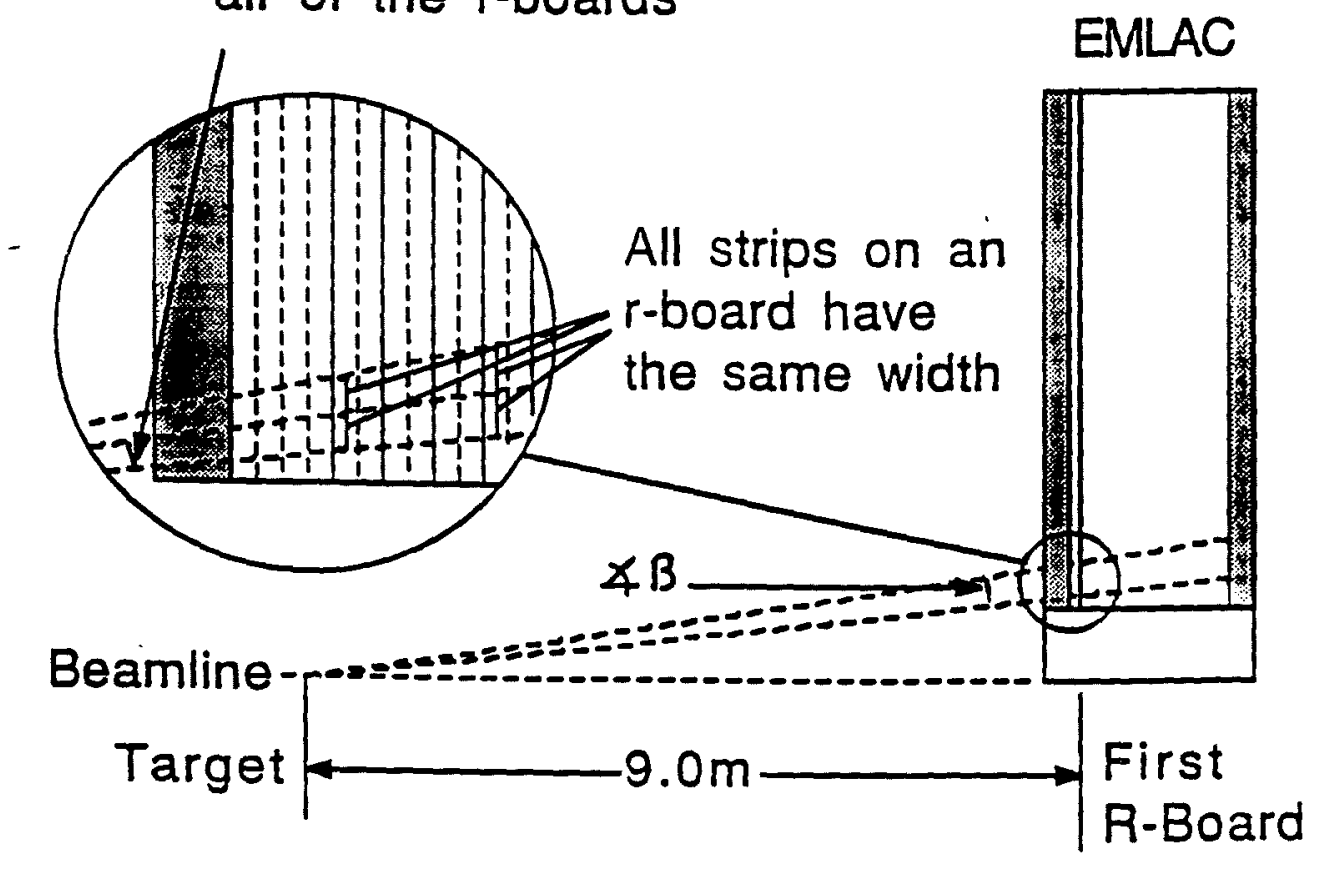

Figure 2.9: $\mathrm{R}$ strips and focussing effect 
in each of the front and back sections and each of them subtends an angle of $\pi / 192$ radians at the target. The other types, called the "outer $\phi$, , run from a radius of $40 \mathrm{~cm}$ out to the outer edge of the anode board. There are 192 outer- $\phi$ strips per quadrant in each front and back section and each of these subtends an angle of $\pi / 384$ radians.

This geometry covers a region of -1.0 to +1.0 units in center of mass rapidity for a beam energy of $530 \mathrm{GeV}$. It has full azimuthal coverage except at the quadrant and octant boundaries.

\section{Characteristics of the electromagnetic calorimeter}

The radiation length of the lead plates $(0.56 \mathrm{~cm})$ is relatively short compared to its interaction length $(17.2 \mathrm{~cm})$. Hence, electromagnetic particles such as photons and electrons shower quickly and deposit their energy in a short distance along their direction of motion. In contrast, hadronic particles have a larger longitudinal shower length relative to electromagnetic particles.

The energy resolution is limited by fluctuations introduced by sampling the detected energy. The lead plates are $2 \mathrm{~mm}$ thick and the argon gap between them is $2.5 \mathrm{~mm}$ thick. The sampling fluctuations yield:

$$
\frac{\sigma(E)}{E}_{s}=\frac{8.15 \%}{\sqrt{E}}
$$

where:

$\mathrm{E}=$ Energy in $\mathrm{GeV}$

s refers to sampling fluctuations

$\sigma(E)=$ error in the energy in $\mathrm{GeV}$ 
In addition to this there are variations in the measured energy from path length fluctuations of the showering particles and fluctuations in the way charged particles deposit their energy. This gives rise to:

$$
\frac{\sigma(E)}{E}_{L}=\frac{2}{\sqrt{N} \ln (10000 x)}
$$

where:

$x=$ thickness of a detector cell (one layer of lead and argon gap) $=3.28 \mathrm{~g} / \mathrm{cm}^{2}$

L refers to Landau fluctuations

and

$N=$ number of samples

When this is combined with the sampling fluctuations due to the thick layer of active argon medium, the resultant energy resolution is:

$$
\frac{\sigma(E)}{E}=\frac{8.3 \%}{\sqrt{E}}
$$

In Figure 2.10 the difference in the energy of electrons of various energy, measured in the $\mathrm{r}$ and $\phi$ strips is plotted. The width of this distribution is indicative of the energy resolution of the calorimeter. In Figure 2.11 the uncertainty in the measurement of the energy is plotted against the energy of the calibration beam electrons. As can be inferred from this figure, the measured energy resolution is:

$$
\frac{\sigma(E)}{E}=\frac{14.0 \%}{\sqrt{E}}
$$

This increase is due to the fluctuations associated with the electronics of the amplifiers and other components of the calorimeter data readout system. 

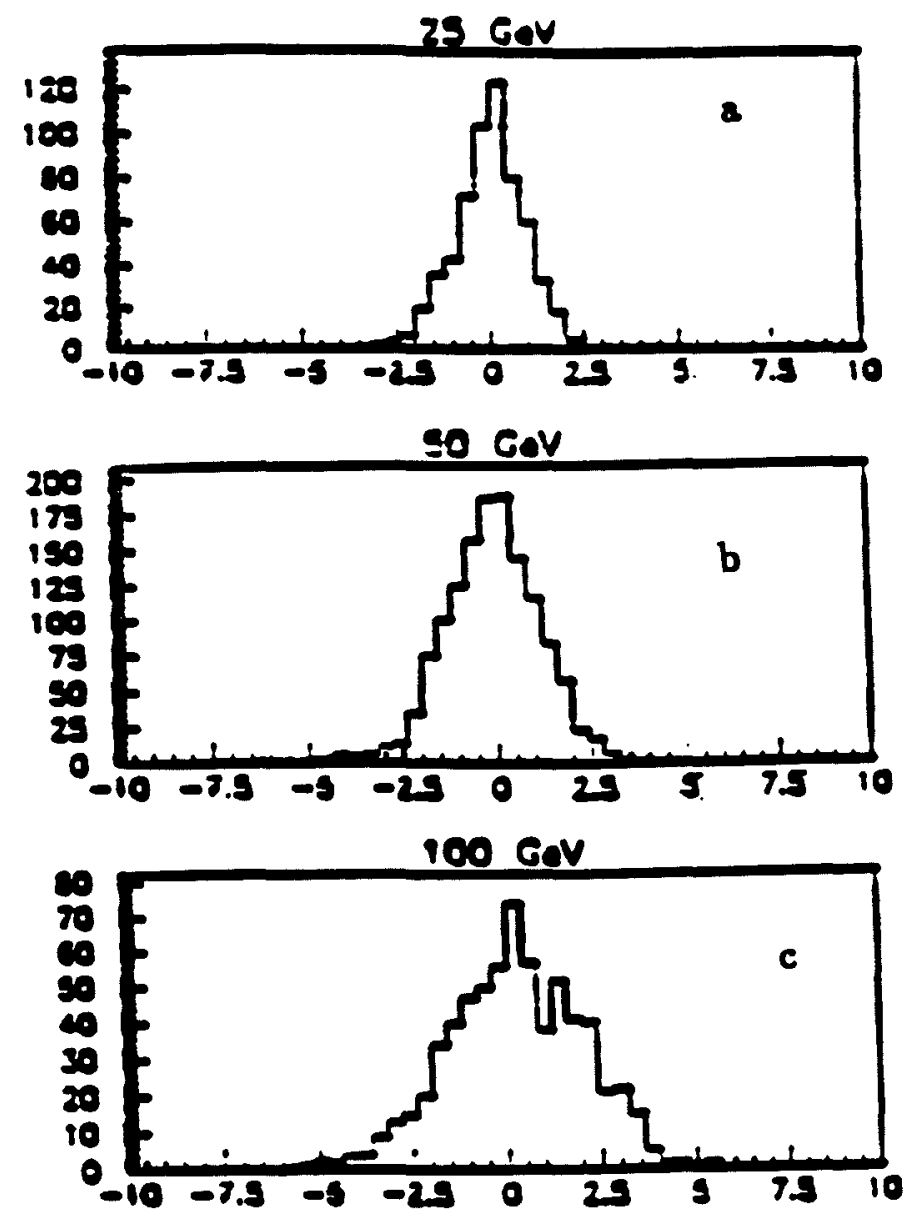

\section{$\mathrm{GeV}$}

Figure 2.10: Energy difference in $\mathrm{R}$ and $\phi$ of electrons at (a) $25 \mathrm{GeV} / \mathrm{c}$ (b) $50 \mathrm{GeV} / \mathrm{c}(\mathrm{c}) 100 \mathrm{GeV} / \mathrm{c}$ 
The position resolution for the r-strips is $0.19 \mathrm{~cm}$ and the angular resoIution for $\phi$ strips is 0.5 mrad. This is one of the most important parameters of this detector. As an example, the minimum separation between two photons from the decay of a $\pi^{0}$ of $100 \mathrm{GeV} / \mathrm{c}$ at $9 \mathrm{~m}$ from the target is about $2.5 \mathrm{~cm}$. Hence they should be easily resolved by the EMLAC. In Figure 2.12 the difference in the location of the electromagnetic shower at the front of the calorimeter and that of the projection of the tracks in the front of the calorimeter is plotted. From the width of this distribution if the projection error of the track is subtracted, the uncertainty in the shower location can be obtained. This is estimated to be $0.19 \mathrm{~cm}$.

The timing resolution of the EMLAC is about $6 \mathrm{~ns}$. This is a measure of the limit in time between which one can reliably separate two different events. The EMLAC can sustain interaction rates of the order of $10^{6}$ particles/s.

\subsubsection{The hadron calorimeter (HALAC)}

\section{Geometry of the hadron calorimeter}

The HALAC has 52 steel plates, each plate being $2 \mathrm{~cm}$ thick. In between them, there are detector planes called "cookies" which contain the charge collecting pads. Liquid argon again fills the region between the cookies. A detector cell consists of a cookie and a steel plate. The calorimeter is again divided into front and back sections. The front section has 14 cells 


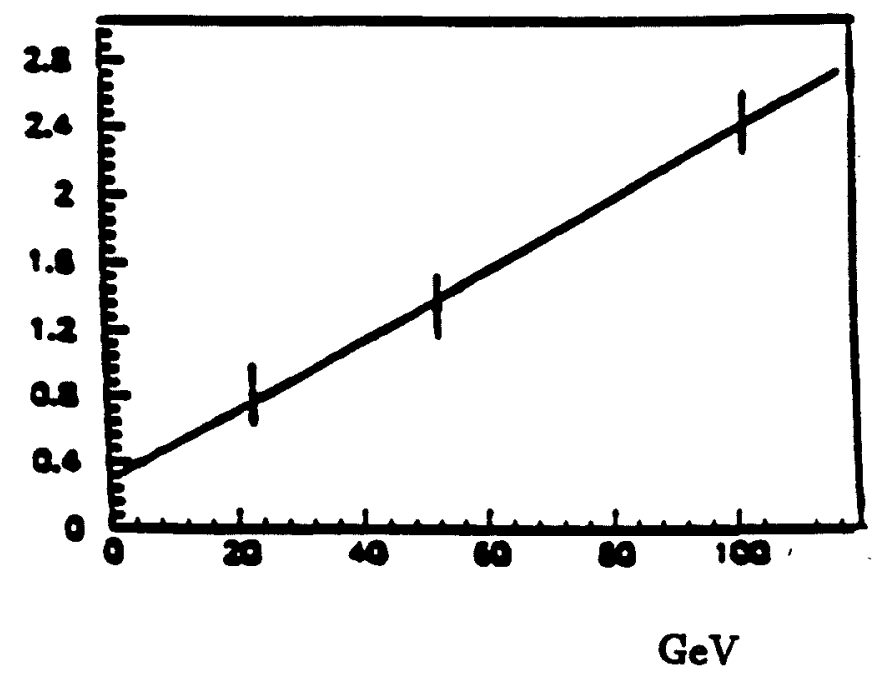

Figure 2.11: Energy uncertainty versus energy of electrons 

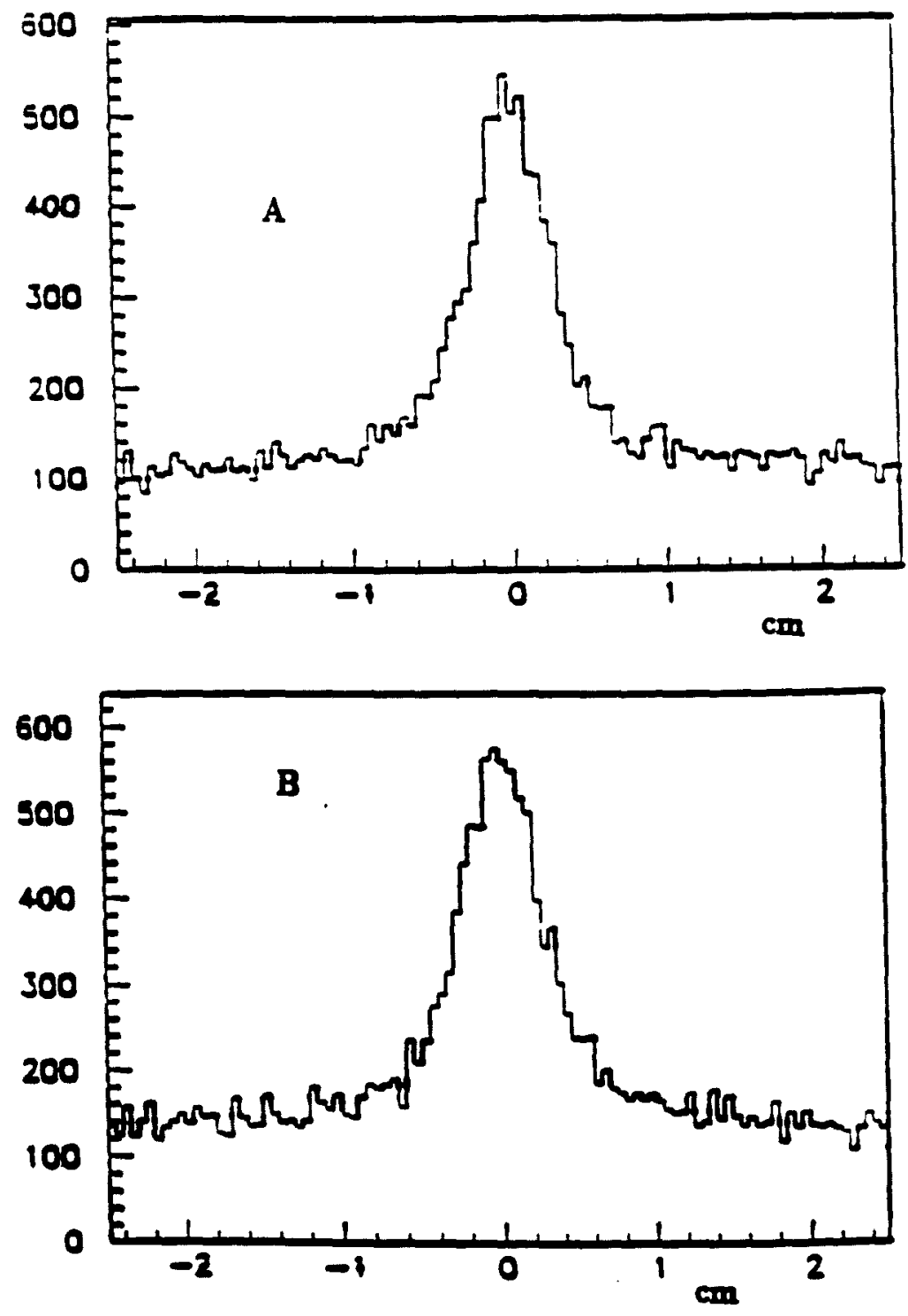

Figure 2.12: Difference in the location of electromagnetic showers and the projection of charged tracks to the front of EMLAC 
(corresponding to 2.6 interaction lengths) and the back section has 39 cells (corresponding to 7.2 interaction lengths).

The cookies are laminates of four layers of $0.8 \mathrm{~mm}$ thick copper clad G-10 interleaved with strips of $3.2 \mathrm{~mm}$ thick G-10. Each layer is made of $3.65 \mathrm{~m}$ by $1.22 \mathrm{~m} \mathrm{G}-10$ sheets. The outer two layers are double sided G-10 and serve as high voltage planes. The inner two layers are the anode planes and they are made of single sided G-10. The side of the high voltage plane facing the anode pads is connected to the high voltage and the other side is grounded.

The anode planes are tessellated into pads which are equilateral triangles (Figure 2.13). These triangles can be combined into hexagons which form constant intervals of rapidity and azimuth for the geometry of the experiment.

The pads are etched on to the G-10 in rows, with their readout lines between the rows, thus creating regions in the plane where no charge is collected. In order to compensate for this, the two adjacent anode planes have conjugate sets of pads so that when an area in one plane has no charge collection, the same area in the following conjugate plane does have charge collection. This provides a uniform coverage of the solid angle subtended by the calorimeter. The readout lines on each anode board are covered with G-10 strips so that any extraneous charge is not collected by the lines, which might otherwise cause unwanted signal cross-talk between the pads.

In a manner similar to the case of the r-strips in the EMLAC, the pad 


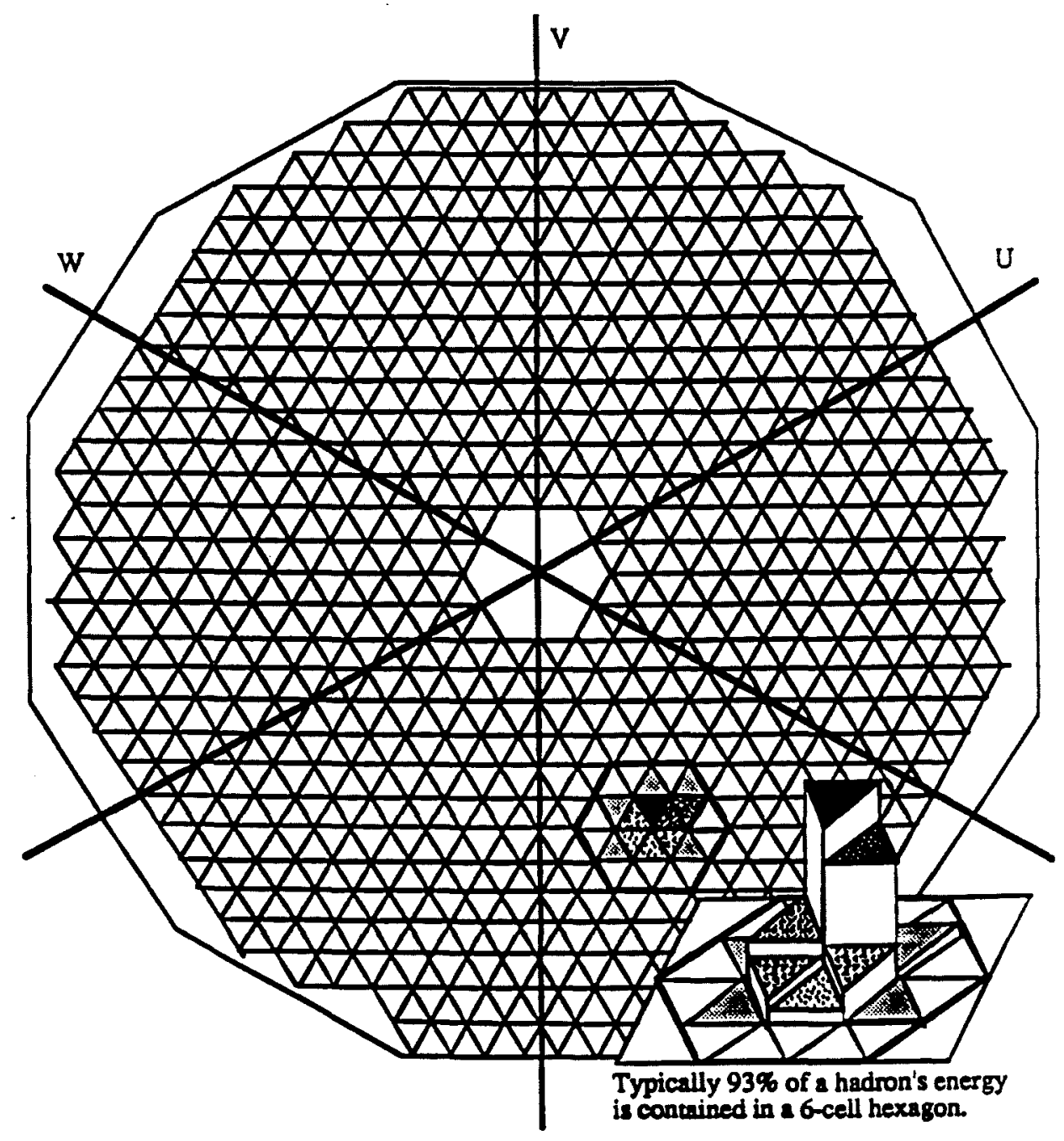

Figure 2.13: Picture of the triangular pads 
sizes in the HALAC grow according to their radial position so that they also focus at the target. In this way, the lengths of the side of the triangles vary from $10.8 \mathrm{~cm}$ to $13.7 \mathrm{~cm}$.

\section{Characteristics of the hadron calorimeter}

Charged hadrons spend about $2 / 3$ of their energy compensating for the binding energy involved in the breakup of nuclei. Since this energy is never detected, the attainable resolution is restricted. The amount of absorber material needed for $99 \%$ containment of a hadronic shower is given by the formula [17]:

$$
L=A(0.54 \ln (E)+0.4) \lambda_{i}
$$

where:

$\mathrm{E}=$ energy of the shower in $\mathrm{GeV}$

$\mathrm{A}=2.5-3.0$

$\lambda_{i}=$ interaction length of the material $=16.8 \mathrm{~cm}$ for steel

$\mathrm{L}=$ length of the material

For $A=3.0$ and $99 \%$ energy containment and $\mathrm{E}=100 \mathrm{GeV}$, we need $L=9.78 \lambda_{i}$. Since 2.03 interaction lengths of material are already in EMLAC, one needs 7.75 interaction lengths in HALAC. This comes to about $130 \mathrm{~cm}$ of steel. Actually, the HALAC has 52 steel plates each of which are $2.5 \mathrm{~cm}$ thick corresponding to a total of $130 \mathrm{~cm}$.

The energy resolution of the hadron calorimeter can be parameterized 
as:

$$
\frac{\sigma(E)}{E}=\sqrt{\left(\frac{0.5}{\sqrt{E}}\right)^{2}+\left(0.4 \sqrt{\frac{4 t}{3 E}}\right)^{2}}=\frac{69 \%}{\sqrt{E}}
$$

for the hadron calorimeter geometry of E706, where:

$\mathrm{E}=$ energy in $\mathrm{GeV}$

$t=$ detector cell thickness in units of interaction lengths.

\subsection{Forward calorimeter}

The forward calorimeter is designed to measure the energy of the particles which go through the central hole of the liquid argon calorimeter.

The forward calorimeter [5] consists of three cylindrical modules positioned coaxially along the beamline. Each of the modules has an outer radius of $114.3 \mathrm{~cm}$ and an inner beamhole radius of $10.16 \mathrm{~cm}$. It contains alternating layers of iron and plexiglass doped with scintillator in planes perpendicular to the beam. Each layer of iron is $1.91 \mathrm{~cm}$ thick and each layer of scintillator is $0.48 \mathrm{~cm}$ thick. The two upstream modules each contain 28 layers of iron and 29 layers of scintillator. The last module contains 32 layers of iron and 33 layers of scintillator. The forward calorimeter has a total of 10.5 interaction lengths of material. There are 76 light pipes inserted through these layers, parallel to the beam line. They have a diameter of $1.11 \mathrm{~cm}$. Figure 2.14 shows one module of the forward calorimeter.

The outer rim surface and the surface of the beam hole were coated with scintillator paint to boost the light reflection efficiency. The scintillation light collected in the light pipes is sent to photomultipliers which are 
connected to the data acquisition system. By measuring these photomultiplier signals and knowing the location of the light pipes, one can determine the energy deposited in the calorimeter.

\subsection{E672 and muon detection}

Experiment E672 is studying[14] $\mathrm{J} / \psi$ production in hadronic interactions. They use the E706 apparatus with additional muon detection spectrometer. This muon spectrometer is located downstream of the forward calorimeter. Figure 2.15 shows a picture of the E672 spectrometer. This consists of a toroid magnet with an average $P_{T}$ kick of $1.3 \mathrm{GeV} / \mathrm{c}, 15$ planes of proportional wire tracking chambers and 2 planes of scintillator hodoscopes with associated electronics to form a dimuon trigger. E672 collected their data at the same time as E706 but using an independent trigger. The data from the E672 spectrometer were available to both experiments.

The acceptance of this spectrometer was good only for positive $x_{F}$ and was maximum at an $x_{F}$ of 0.25 . The momentum of the muon tracks identified in E672 was measured using the tracking system in E706. The measurement of the $\mathrm{J} / \psi$ mass (see Section 5.1.7) done this way has confirmed the validity of the momentum scale used to determine the momentum of the charged tracks in the tracking system in E706. 

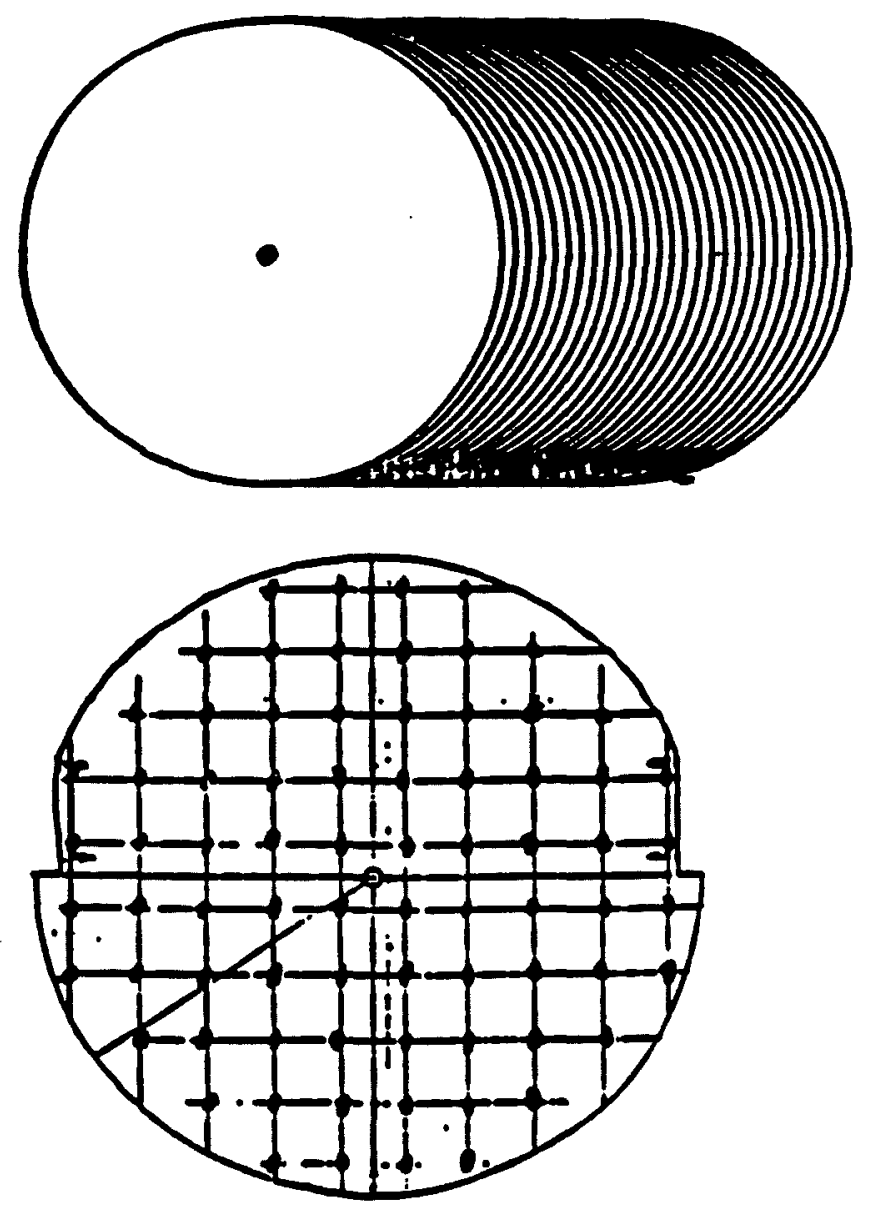

Figure 2.14: Picture of the forward calorimeter 


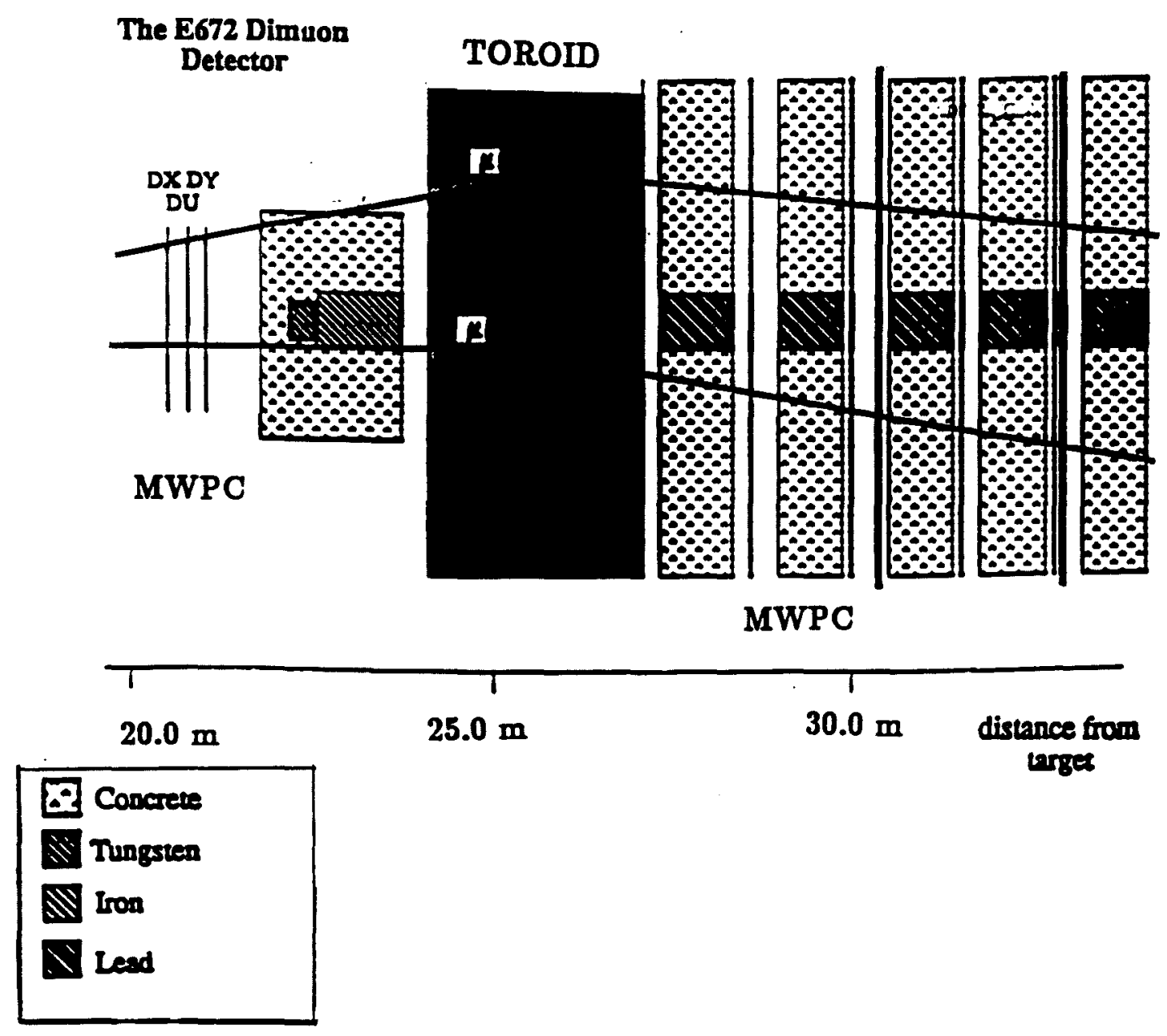

Figure 2.15: E672 MWPC, toroid, dimuon event picture 


\section{Chapter 3}

\section{E706 Multiwire Proportional Chambers}

\subsection{Construction of the E706 MWPC's}

\subsubsection{General construction considerations}

In this chapter some of the details are described of how two (Chambers I and II) of the four MWPC's were constructed and instrumented at Penn State. The other two MWPC's (III and IV) were built and instrumented at Michigan State University using similar techniques. In Appendix B the design considerations of MWPC and the principles of MWPC operation are summarized.

\subsubsection{Frames for cathodes and anodes}

Both cathodes and anodes are constructed using G-10 frames for support. These are strong and non-conducting.

The cathode plane is made of mylar stretched under tension.

The anode planes have wires which are also under tension. Hence in order for the G-10 frames not to bend under the stress, 
the cathodes and anodes are supported on steel pins passing through two strong metallic frames on the outside.

\subsubsection{Cathode construction}

The size of the cathodes is determined from the acceptance requirements of the whole spectrometer. Since the four MWPC's are located between $360 \mathrm{~cm}$ and $700 \mathrm{~cm}$ downstream from the target, each plane should increase in size as the distance from the target increases. However for ease of construction, the sizes of the cathode and anode planes are the same for all planes in a given MWPC. Instead, the sizes of the entire chamber increase as one goes downstream. Table 2.3 shows the sizes of the cathodes.

\section{Mylar stretching and graphite spraying}

Each cathode is made of $0.0254 \mathrm{~cm}$ thick mylar. In order to give the desired surface tension, this mylar was stretched on a stretching machine. Figure 3.1 shows the picture of a completed cathode on the stretching machine. A G10 frame was glued onto the mylar after stretching. This whole assembly was kept on a temporary aluminium frame during cathode construction. Graphite was sprayed onto this in order to make the cathode conductive. An automatic spray machine was used for this purpose so that a uniform thickness of graphite is obtained on the cathode. The surface resistance was measured at various parts of the cathode in order to ensure the uniformity of the cathode in various regions. 


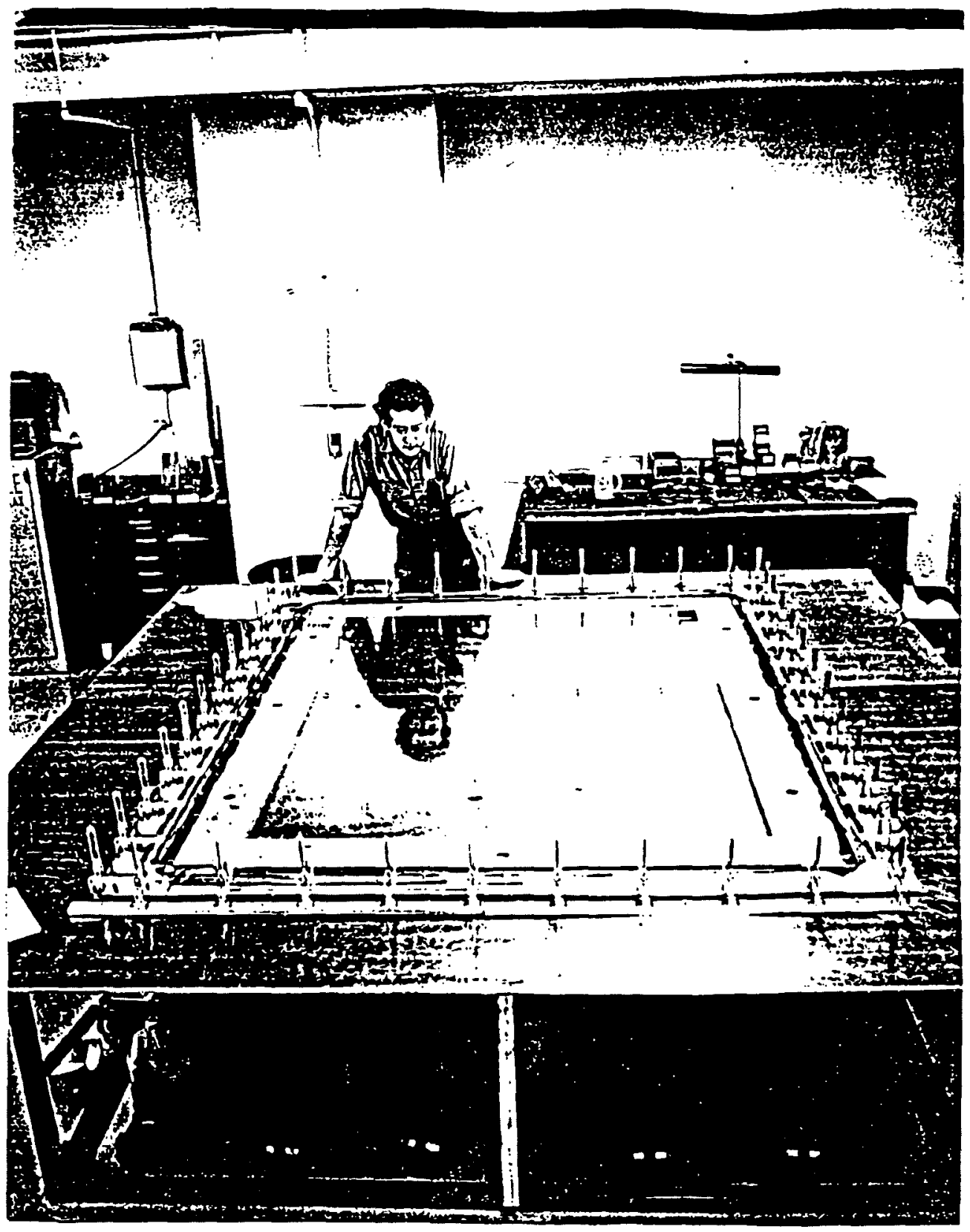

Figure 3.1: Picture of a completed cathode 


\section{Regions of the cathode}

The central region of the MWPC is exposed to the beam fragments of interactions as well as non-interacting beam particles during data taking. The intensity of the particles in this region is such that the produced ionsation can cause damage to the anode wires which go through that region if they are in high electric fields. For this reason, the high voltage applied between cathode and anode was kept lower in that region. To achieve this, the graphite covered cathode contains three regions. The inner one is a square of side $3.8 \mathrm{~cm}$ and is called the "beam region." Outside this there is the "diffractive region," which is a square of side $20.3 \mathrm{~cm}$. The rest of the cathode is called the "normal region." These three regions are electrically isolated with respect to each other so that different voltages can be applied to these regions separately.

The electrical connection to the cathode is made by making a small hole in the cathode and gluing a small aluminum foil in the hole from the nonconducting face of the anode. This operation was done for all three regions of the cathodes before spraying graphite.

\section{Garlands (support wires)}

In order to preserve a constant distance between the cathode and anode, the planes were constructed with some mechanical spacers between them. This prevents the cathode from deflecting into the anode while in operation. A detailed discussion of this aspect is given in Appendix B. Zigzag-shaped 
kapton tapes called garlands were attached to the face of the cathode. Figure 3.2 shows how this is done.

However the introduction of the garlands distorts the field configuration around them. In order to compensate for this, an insulated conductive wire runs along the garland (see Figure 3.2). The ends of this wire were soldered on to their connectors at the edge of the cathode. The field due to a small current going through this wire is designed to compensate the field distortion due to the garland.

\section{Assembly of the cathode}

After checking the quality of all electrical connections and graphite spray, each completed cathode was mounted onto the main frame of the MWPC and the temporary aluminium frame was removed.

\subsubsection{Anode construction}

\section{Anode frame construction}

The frame of each anode is made of G-10 boards. These boards were glued together to make a window frame. The printed circuit(PC) boards for reading out the signals were constructed by an outside firm. These boards were then glued onto the G-10 frame. All the gluing had to be done with precision in order to avoid any irregularities in the structure of the frame and in the location of the printed circuit boards. A gluing jig was made out of wood with the appropriate shape to do all the gluing for the frames. 


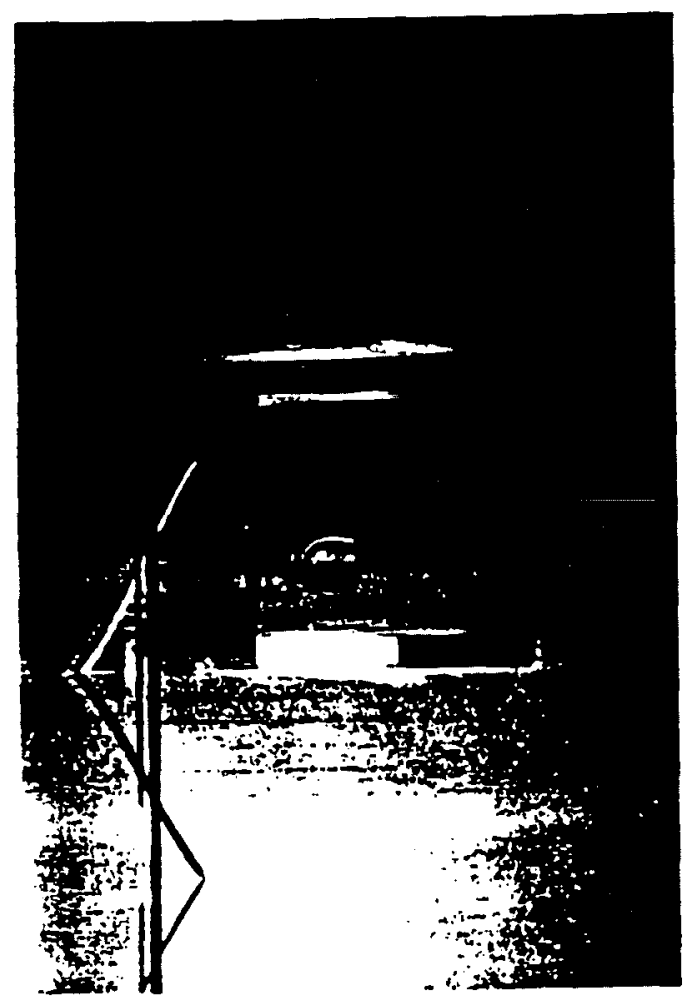

Figure 3.2: Garlands 


\section{Wire laying on the anode}

Following the construction of the anode window frames, the anode wires were attached. The wires are made of gold-plated tungsten. They have a diameter of $203 \mu \mathrm{m}$. This material was chosen since it does not react or disintegrate in the high electric fields used in the MWPC.

The spacing between the wires is $2.54 \mathrm{~mm}$ and the wires have a tension of $40 \mathrm{mg}$ in order to prevent them from deflecting in the field. In order to preserve the uniformity of the field, it is important to keep the spacing between adjacent wires a constant and to keep all wires in one plane. A wire laying machine was designed and constructed for this purpose (see Figure 3.3). The G-10 anode frame was mounted on a temporary aluminium frame and kept horizontal on this machine with the printed circuit boards facing up. The orientation of the frame in the horizontal plane of the machine depended upon which view ( $\mathrm{X}, \mathrm{Y}, \mathrm{U}$ or $\mathrm{V}$ view) was being made.

Each wire was laid on the frame an equal distance apart. A $40 \mathrm{~g}$ weight was hung at the end of the wire to give the correct tension. The ends of the wire were then soldered onto the appropriate printed circuit board channel. A telescope was used on each end of the wire to ensure the correct location of the wire. A few wires near the edge of each frame had $254 \mu \mathrm{m}$ thickness. The reason for this was that at the edges the field was going to be more varying and hence only thicker wires would be able to withstand the deflecting forces. The location of the wires with respect to the physical center of each G-10 board was done with an accuracy of a few microns. 


\section{Assembly of the anode plane}

After the wires were soldered on the frame, the stability and quality of the soldering of each wire was checked and all the faulty ones were redone. Epoxy was subsequently poured over the solder joints in order to give more strength and durability to the wire connection. This rubber-like epoxy allowed a little motion of the wire, thereby reducing the chance of breaking the wire. The completed anode was mounted onto the main frame of the MWPC and the temporary aluminium frame was then removed.

\subsubsection{Spacing between the various planes}

The spacing between the various planes was kept at $0.57 \mathrm{~cm}$. This distance was matched to the anode wire spacings for optimum electric field strength of the MWPC's. The dimensions and the numbers of wires are summarized in Table 2.3. A total of 14000 wires (channels) are involved in the coordinate measurements of the 16 planes of the MWPC's.

\subsection{Operation of the E706 MWPC's}

Around the sides of each chamber there are gas boxes which allow the flow of gas through the chamber volume. They are insulated from the outside so that air does not enter the system. On the two faces of the MWPC, a plane of aluminised mylar provides the insulation. The appropriately mixed gas mixture entered the chamber through a valve in the gas box on the top and it left the system through a valve in the gasbox at the bottom. The 


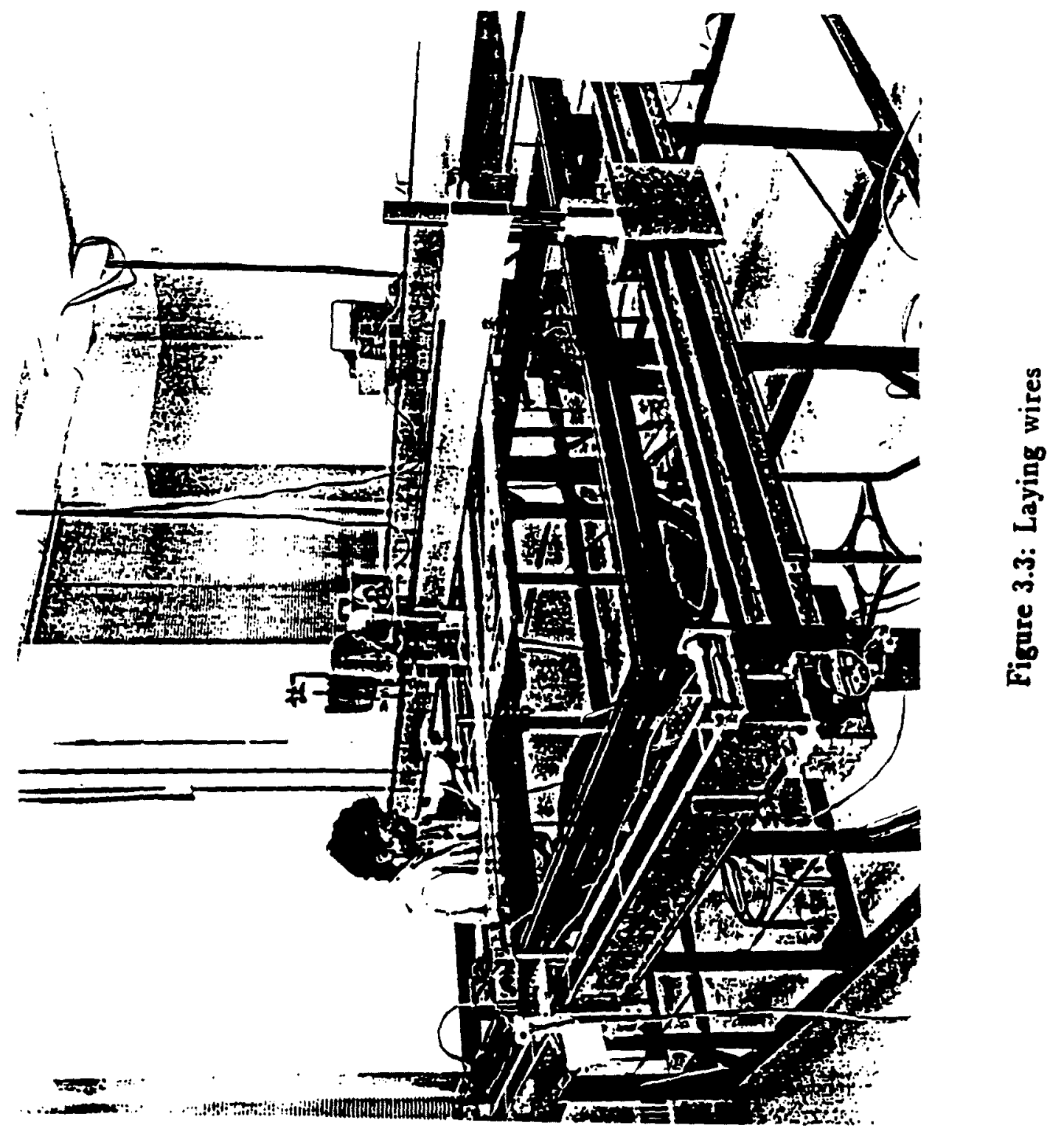


gas pressure in the MWPC's was maintained by adjusting the flow rate into and out of the system. In this experiment a modified version of the so called "magic gas" was used. It contains $79.7 \%$ argon, $18.0 \%$ isobutane, $0.1 \%$ freon and $2.2 \%$ alcohol. These gases were premixed before entering the MWPC. The gas mixture in the MWPC was at atmospheric pressure. The gas flow rate was $1.51 /$ hour. The gas flow system and the pressure were monitored continuously and an alarm system was triggered if the pressure in the MWPC's dropped below the desired amount.

\subsubsection{Readout electronics}

The current read from the wires was amplified for the readout. The voltage was set such that the chambers operated in proportional mode, so that the charge collected per wire is proportional to the charge of the particle which caused the charge avalanche.

The typical values for the high voltage and threshold voltages used are given in Table 3.1 .

\subsubsection{Gate width and readout delays}

The gate width (the amount of time the MWPC is readout for each event) was set such that it reads only minimum ionizing particles. The gate width is 100 ns. The delay for the signal to reach from the MWPC to the electronics racks was $700 \mathrm{~ns}$.

A measure of the efficiency of the MWPC can be obtained by keeping it in the beam with high voltage applied. The ratio of the number of particles 
Table 3.1: Typical voltages for MWPC operation

\begin{tabular}{|c|c|c|c|}
\hline $\begin{array}{c}\text { Chamber } \\
\text { number }\end{array}$ & $\begin{array}{c}\text { High } \\
\text { voltage }(\mathrm{kV})\end{array}$ & $\begin{array}{c}\text { Garland } \\
\text { voltage }(\mathrm{kV})\end{array}$ & $\begin{array}{c}\text { Threshold on } \\
\text { amplifiers (V) }\end{array}$ \\
\hline 1 & 2.8 & 1.0 & 1.5 \\
2 & 2.8 & 1.0 & 1.5 \\
3 & 2.8 & 1.0 & 1.5 \\
4 & 2.8 & 1.0 & 1.5 \\
\hline
\end{tabular}

detected by the MWPC to that of the actual number of beam particles which went through the system is a measure of the efficiency of the chamber. These measurements were performed at various values of the high voltage applied. As the voltage applied increases, the efficiency increases and then remains at a constant (plateau) for a certain range of voltage. The high voltage corresponding to the beginning of this plateau is the operating voltage of MWPC. A typical plateau curve obtained for one of the planes of each chamber is shown in Figure 3.4.

\subsubsection{Read-out electronics}

The inputs to the amplifier cards are connected through a cable to the printed circuit boards on the anode plane. This cable goes through the insulator of the gas system. The output of the amplifier is connected to a readout cable coupled to the latches and Camac controller. The data acquisition computer was finally interfaced to the Camac controllers. 

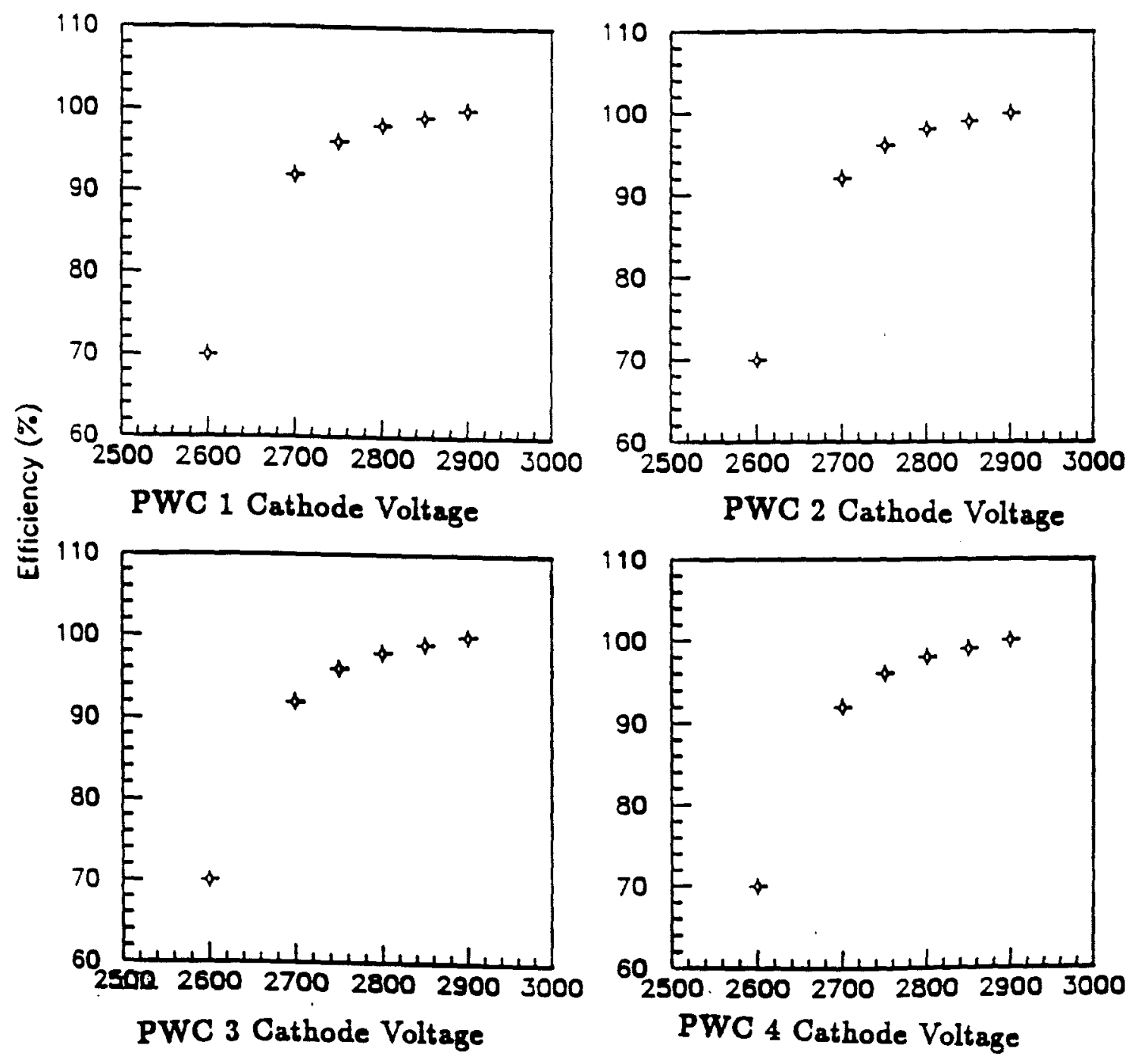

Figure 3.4: Typical MWPC plateau curves 


\section{Chapter 4}

\section{Trigger and Data Acquisition}

\subsection{Description of the trigger system}

Since the production cross sections for direct photons fall exponentially, one needs a trigger which selects high transverse momentum events. Hence the basic purpose of the trigger system [32] was to collect events which had at least one high transverse momentum electromagnetic shower deposited in the electromagnetic calorimeter. This system was designed for interaction rates up to $10^{6}$ per second.

The trigger selection of an event was done in three main steps, namely beam and interaction definition, preselection of the interaction and final selection of the event.

\subsubsection{Beam and interaction definition}

Two thin plastic scintillation counters were located on the beam line directly upstream of the target. A beam particle signal was defined as the coincidence of the signals from these two counters. 
There were four scintillation counters located downstream of the target. They covered the angular acceptance region of the electromagnetic calorimeter (EMLAC). The following requirements were imposed on the output of these counters to have an interaction qualified for preselection.

- These counters were put in coincidence with the beam signal.

- When an interaction occurred, it was required that there be no other interaction occurring within $\pm 60 \mathrm{~ns}$ of that interaction. This prevented overlapping (pile-up) of signals from different events due to insufficient time resolution. This filtering of events is called the pileup filter.

- The interaction should not be due to a halo beam particle incident on the target. In order to achieve this, a third beam counter was placed upstream of the target. This counter had a hole in the center and hence it recorded only the presence of the halo beam particles. The signal from this counter was used in anticoincidence with an interaction which satisfied the pile-up filter.

If all of the above requirements were satisfied, an "interaction strobe signal" was generated.

\subsubsection{Preselection of an interaction}

A further selection was made based on the transverse momentum of the showers in the electromagnetic calorimeter (EMLAC). A so-called "octant 
global $p_{T}$ " signal was generated in each octant of the EMLAC by weighting the energy deposited in the r-view (circular strips) of the octant according to the radial position of the showers. The octant global $p_{T}$ is the total $p_{T}$ deposited in an octant. A minimum octant global $p_{T}$ of $1.7 \mathrm{GeV} / \mathrm{c}$ was required in at least one octant for event selection.

Since the rise time of the EMLAC signals was of the order of $300 \mathrm{~ns}$, one had to prevent the possibility that the global $p_{T}$ signal was due to the sum of two low $p_{T}$ depositions from distinct events in that time range. For this the octant global $p_{T}$ was put in coincidence with the "interaction strobe signal" mentioned above and in anticoincidence with discriminated global $p_{T}$ signals present within the preceding $300 \mathrm{~ns}$. An "octant pretrigger signal" was generated if all this logic was satisfied.

Any muons coming parallel to the beam would be incident on one of the quadrants of the two veto walls which in turn generated signals. A 150 ns wide "veto signal" was created when there was a coincidence of the signals from any two geometrically corresponding quadrants of the two veto walls. Some of the $400 \mathrm{~Hz}$ power supplies created a signal of width $30 \mu \mathrm{sec}$. Another "veto signal" was created in coincidence with this signal.

A "pretrigger signal" was generated in coincidence with the logical OR of all "octant pretrigger signals" and in anticoincidence with the two "veto signals" mentioned above. The creation of this signal qualified the event to be preselected.

This signal was also used to latch the MWPC, SSD and forward calorimeter data. If this pretrigger signal was not generated, a reset signal was sent 
Table 4.1: Typical threshold setting for triggers

\begin{tabular}{|l|l|l|l|}
\hline GLB P $_{T} \mathrm{HI}$ & GLB P $_{T} \mathrm{LO}$ & $\mathrm{LCL} \mathrm{P}_{T} \mathrm{HI}$ & $\mathrm{LCL} \mathrm{P}_{T} \mathrm{LO}$ \\
\hline $3.6 \mathrm{GeV} / \mathrm{c}$ & $2.5 \mathrm{GeV} / \mathrm{c}$ & $3.0 \mathrm{GeV} / \mathrm{c}$ & $1.2 \mathrm{GeV} / \mathrm{c}$ \\
\hline
\end{tabular}

out and the trigger system was then ready to consider the next interaction.

\subsubsection{Final selection of the event}

The global $p_{T}$ signal could also be generated from coherent noise (noise common to all channels) or from multiphoton events in which each photon has only a small fraction of the total $p_{T}$. It is desirable to avoid recording these types of events. The fine granularity of the EMLAC was used to suppress triggers of this kind by imposing a "local $p_{T}$ " condition. This requires that a substantial fraction of the global $p_{T}$ in an octant be deposited within 16 adjacent $\phi$ strips of the calorimeter, roughly the size of a normal electromagnetic shower. A "local $p_{T}$ signal" is generated if this condition is satisfied.

Each of the two $p_{T}$ signals (global and local) from each octant was discriminated using different nominal thresholds. Using the two $p_{T}$ signals and the different nominal thresholds, four EMLAC triggers were implemented. They are called "local low - global high," "local low - global low," "single local hi" and "two - $\gamma$ low." Table 4.1 shows the typical threshold settings for these triggers.

The "local low - global high" trigger was produced if one or more octants 
had a "local $p_{T}$ signal" which satisfied the lower $p_{T}$ threshold and a "global $p_{T}$ signal" which satisfied the higher $p_{T}$ threshold.

The "local low - global low" was similar to "local low - global high," except that the "global $p_{T}$ signal" was required to satisfy only the lower threshold. Since most of the events were at low $p_{T}$ the "local low - global low" trigger was suppressed (prescaled) by a factor of ten to avoid the dominance of low $p_{T}$ events selected.

The "single - local hi" trigger required that at least one octant had a "local $p_{T}$ signal" which satisfied the higher $p_{T}$ threshold. The "two $-\gamma$ low" trigger required that if an octant had a local $p_{T}$ signal which satisfied the lower threshold, any of the three diametrically opposite octants must have a "local $p_{T}$ signal" which also satisfied the lower threshold.

Each of the four triggers mentioned above required that both the pretrigger signal and the "octant pretrigger signal" have been produced. If no trigger is formed after the pretrigger signal is formed, the system is reset in 5 microseconds to consider the next event.

When a trigger was actually formed, a signal was sent to the computer to readout the data stored in different parts of the spectrometer. After collecting the data the computer generated a reset signal which was sent to the various readout electronics and the trigger was reset for the next event. 


\subsection{Data acquisition System (DAQ)}

\subsubsection{Data flow}

The main hardware components of the DAQ were a microvax and 4 PDP11 computers. The data from various parts of the apparatus flowed to the memory buffer of one of the PDP-11's. The microvax collected and concatenated the data for each event from the various PDP-11's. These events were in turn written to magnetic tapes. These data were available for online analysis as well. Figure 4.1 shows the configuration of the data flow.

The process of data taking was controlled by gates and pulses generated at different times. The trigger had to perform the task of synchronising the data taking operation at various parts of the detector.

\subsection{Monitoring data}

In order to ensure the quality of the data being taken and to ensure that all parts of the detector were functioning properly, several software tools were used. The data concatenated by the microvax were siphoned off to several programs which monitored the performance of the various parts of the detector continuously.

For the MWPC and SSD systems the number of hits in each plane were monitored in order to make sure that the multipicity in the events were reasonable. In addition, the profiles of the hits in each plane were made in 


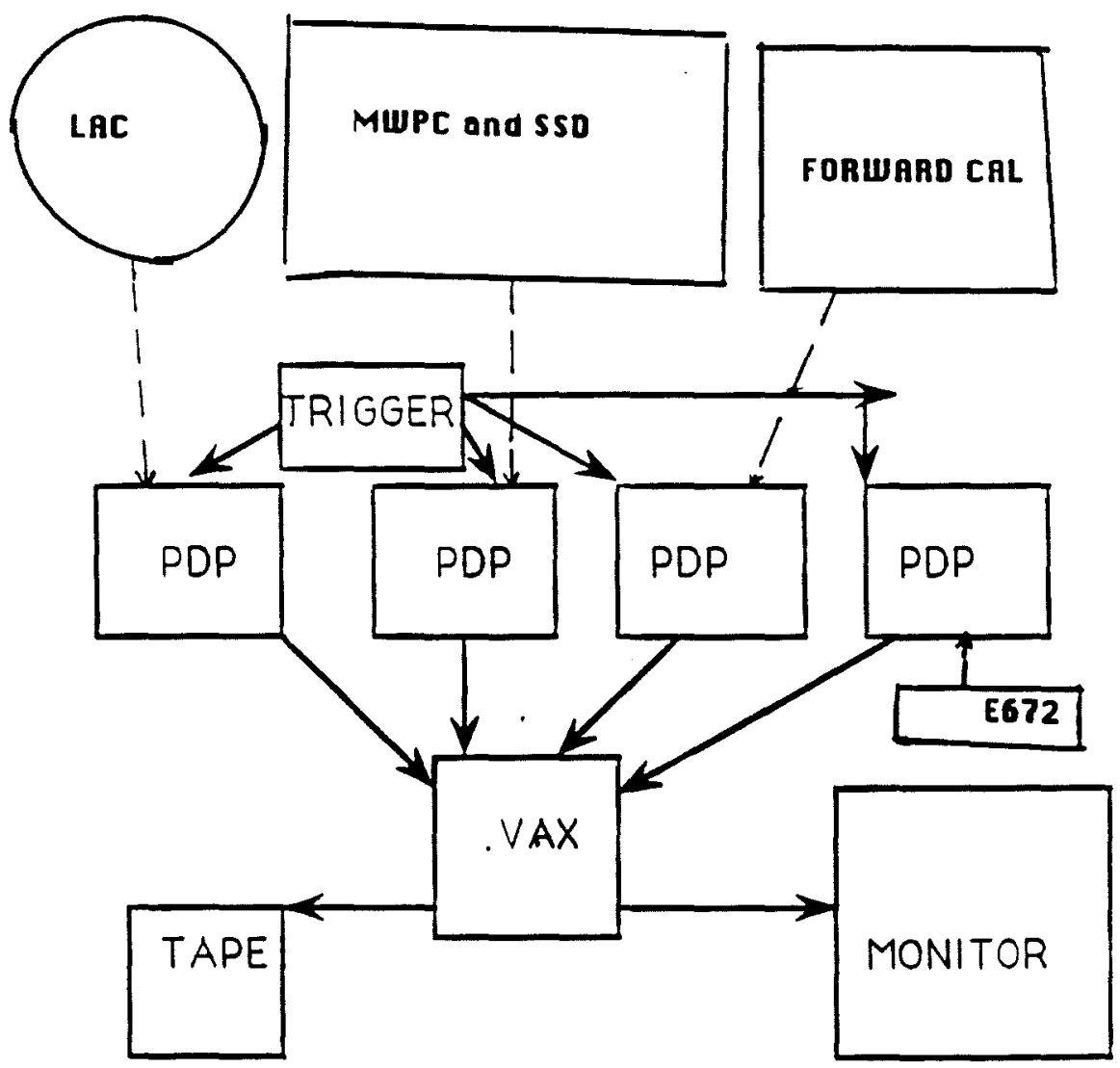

Figure 4.1: Data acquisition flow 
order to look for any hot channels or dead channels in the amplifier output. The high voltages on the planes and all the threshold voltages applied for the amplifiers were monitored all the time.

The energy deposited in various parts of the calorimeter were monitored to ensure that they have reasonable values. There were several counters which measured the number of particles that hit the various scintillation counters. These were also monitored.

The magnetic tapes on which the data were written were processed using an offline reconstruction package and the results of that were also used to check the consistency of the data.

\subsection{Amount of data collected}

In the first run of E706, in 1987-88, a total of about 4.6 million good quality events were triggered and collected on tape. Of these, about 1.9 million events were obtained with a $\pi^{-}$beam and 2.7 million events with a proton $(95 \%)$ and $\pi^{+}(5 \%)$ beam. In Table 4.2 the amount of data collected with the various targets and beams is given. Of these, about 3.1 million events were used for the analysis described in this thesis. Some events were taken just with a trigger requirement that there be an interaction in the target. These events are called minimum bias events and they were used to study the nature of the background to various signals observed in the data. 
Table 4.2: Number of triggers collected

\begin{tabular}{|c|c|c|c|}
\hline $\begin{array}{c}\text { Neg Cu Be } \\
\text { target }\end{array}$ & $\begin{array}{c}\text { Pos Cu Be } \\
\text { target }\end{array}$ & $\begin{array}{c}\text { Neg Be } \\
\text { target }\end{array}$ & Pos Be \\
target \\
\hline 786000 & 1225000 & 1242000 & 1508000 \\
\hline
\end{tabular}




\section{Chapter 5}

\section{Charged Particle Reconstruction}

\subsection{Charged particle tracking}

\subsubsection{General philosophy of downstream tracking}

Since we have four planes in each of the four different views $(X, Y, U, V)$, we first find the view tracks in each of these views. The view tracks are leastsquares straight-line fits to three or four points in the planes of each view. These view tracks are then matched to get straight lines in 3-dimensional space. These are called space tracks.

\section{Algorithm to find view tracks}

The salient features of the logic behind finding view tracks in each view can be described as follows. The input is a set of hits in a plane which are in ascending order. A hit is the coordinate of a wire in a plane which fired when a charged particle crossed it. There are two passes made in searching through the hits. In the first pass the first and fourth planes in the view are taken as what we call "seed" planes. In the second pass, the second and 
third planes, which are the two planes in the middle, are taken as the seed planes. In a given pass, the planes which are not the seed planes are called the "search" planes. A "seed pair" is a pair of hits in which one hit is from one seed plane and the other hit is from the other seed plane.

In the first pass every seed pair is used to try to form a three or four hit track. For each of these pairs there is a slope and intercept for the candidate track. This enables one to get the projection of this straight line onto the search planes. Then one searches through the list of hits in the search planes and all hits within a tolerance $( \pm 1.5$ wire spacing) near the projected position are stored. Here there are two cases. In the first case there are hits in both search planes along the candidate track. This would be the case for a four-hit track. In the second case there are hits in only one of the two search planes. This would be the case for a three-hit track. Least-squares fits using all of the possible combinations of hits stored above are then performed. If a candidate set of hits for a four-hit track fails the $\chi^{2}$ criterion, then a least-squares fit to a three-hit track is made after removing from the set the search plane hit with the worst residue. This first pass is expected to find all possible four-hit tracks and all of the three hit tracks with one of the inner search plane hits missing.

In the second pass the outer two planes become the search planes. The projection from each seed pair from the two inner planes is made and the candidate set of hits along the track in the search planes are stored. Only those combinations which were not originally part of four-hit tracks in the first pass are considered. In this pass the three-hit tracks which missed 
either of the two outer planes are expected to be created. While making three-hit tracks a restriction is made that they should not share more than two hits with a four-hit track.

After making all the tracks, the four-hit tracks are compared among themselves to check on how often they share hits. The set of four-hit tracks which share three or more hits are considered as a global cluster. If the clus-

ter consists of only two tracks, then the one with the higher $\chi^{2}$ is dropped; but if the cluster consists of more than two tracks, the two four-hit tracks which do not share three hits among themselves and which have the two lowest $\chi^{2}$ values are retained and the rest of the tracks in the global cluster are dropped. A similar procedure is followed in comparing three-hit tracks among themselves. Next, the remaining four-hit tracks are compared with the remaining three-hit tracks and all the three-hit tracks which share two or more hits with four-hit tracks are dropped.

This is the procedure to find view tracks in a given view. It is repeated for each of the four views. The output consists of all of the parameters for the view tracks in each of the views. In this algorithm the seed planes can be easily swapped. It is also made as much order-independent as possible. In addition, all of the hits are tagged as to whether they are used in four-hit tracks or three-hit tracks.

\section{Algorithm to find space tracks}

In order to match these view tracks to form space tracks, again two passes are made. In the first pass, the $\mathrm{X}$ and $\mathrm{Y}$ views are taken as the "seed views" 
and the $U$ and $V$ views are taken as the "search" views. In the second pass these roles are reversed. A "track seed" is a pair of riew tracks in which one of the tracks is from one seed view and the second track is from the other seed view. This is a candidate for a space track. For each track seed the candidate space track is projected onto each of the planes of the search views. Then a search is made through each of the lists of hits of the search view planes to find the nearest hit to the projected position, within a tolerance ( \pm 1.5 wire spacing). All such hits from each of the search planes are stored along with the hits which constituted the track seed. In order for this set of hits to be considered for a space track, there should be at least two hits from each view, there should be at least four hits from all of the search views, there should be at least six hits from the planes in the track seed and there should be at least thirteen hits in total. The set of hits which pass all these criteria is subjected to least-squares fitting using all the hits along the track as described below.

\section{Track fitting for space tracks}

Once the candidate hits are found in the various planes for a candidate space track, the view tracks in the seed planes are projected to each of the planes in which there is a hit near the projected position as described in the previous section. A $\chi^{2}$ is now defined as:

$$
\chi^{2}=\frac{1}{N-4} \sum_{i}\left(\frac{\xi_{m s}-\xi_{f i t}}{\sigma_{i}}\right)^{2}
$$


where: $N=$ Number of planes with hit near projected position

$i=$ the plane number

$\xi_{m s}=$ location of the hit in the plane measured in the coordinate system of the plane

$\xi_{f i t}=$ projected location from the view tracks in the seed planes

$$
\xi_{f i t}=\left(a z_{i}+b\right) * \cos \left(\alpha_{k}\right)+\left(c z_{i}+d\right) * \sin \left(\alpha_{k}\right)
$$

where: $k=$ view number $(1,2,3$ and 4 for $X, Y, U$ and $V$ views, respectively). $\alpha=$ angle of the orientation of the view (see Table 2.3).

Also, $a$ and $b$ are the slope and the intercept of the candidate space track in the $\mathrm{X}$ view; $\mathrm{c}$ and $\mathrm{d}$ are the slope and the intercept of the candidate space track in the $\mathrm{Y}$ view.

Minimizing this $\chi^{2}$ gives a set of equations which can be solved for $a, b, c$ and $d$. These quantities define a space track completely.

After creating candidate space tracks from both passes, a track cleanup is performed. If two space tracks share six or more hits, then the one with the lower number of hits is deleted. If they have the same number of hits, the one with the higher $\chi^{2}$ is deleted. The same is true if the number of shared hits plus the number of hits on the adjacent wires exceeds eight. If a track has nine or more hits which are not shared by any other track, then it is always retained. This takes care of the situation in which a particle fires 
more than one wire in several planes and thereby gives rise to more than one candidate space track.

\section{Results of downstream tracking}

Figure 5.1 shows the residues from the tracks on a particular (plane number 20) plane. The width of this distribution shows the position resolution $(2.4$ $\mathrm{mm}$ ) of the MWPC at that plane. In Figure 5.2 through in Figure 5.5 the $\chi^{2}$ of tracks which made hits in $16,15,14$ and 13 planes are shown. This figure demonstrates the quality straight line fits through the hits. The 13 hit tracks were forming the background level to the real track solutions and hence only those 13 hit tracks which had a $\chi^{2}$ below 1.5 were considered. This figure demonstrates the capability of the tracking system in handling multiparticle events. Figure 5.6 shows the relative numbers of $16,15,14$ and 13 hit tracks from which it can be inferred that the majority of the tracks were 15 hit tracks.

\subsubsection{General philosophy of upstream tracking}

The algorithm used to find view tracks in the SSD's is similar to that for the view tracks in the MWPC's. Here we have four planes in each of two orthogonal $(\mathrm{X}, \mathrm{Y})$ views. An additional complication arises in the SSD system, since here one has to take into account the effects of charge sharing by adjacent strips. This is done by clustering of hits in really high multiplicity events. 


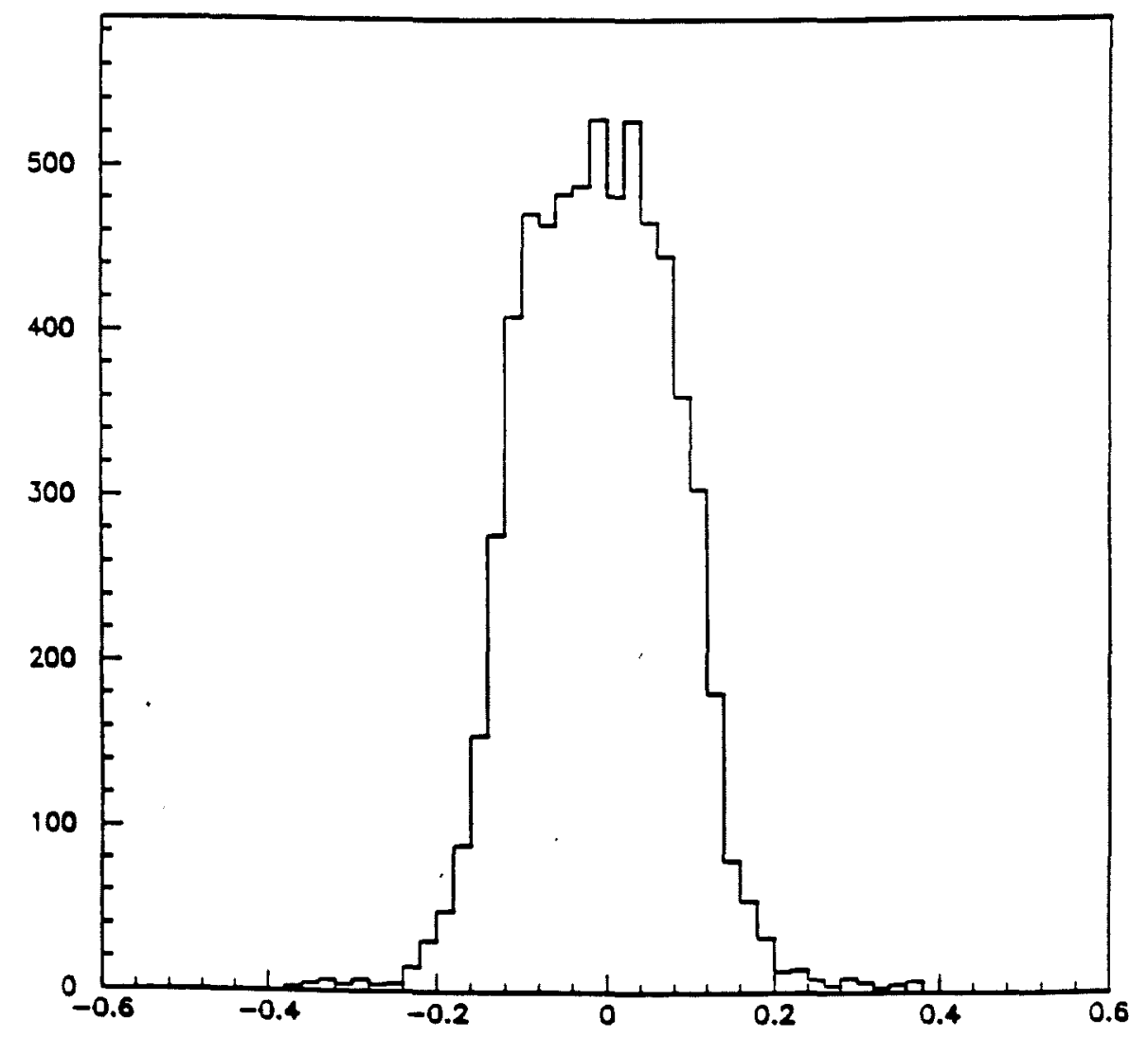

Figure 5.1: Residues from a straight line fit for plane 20 


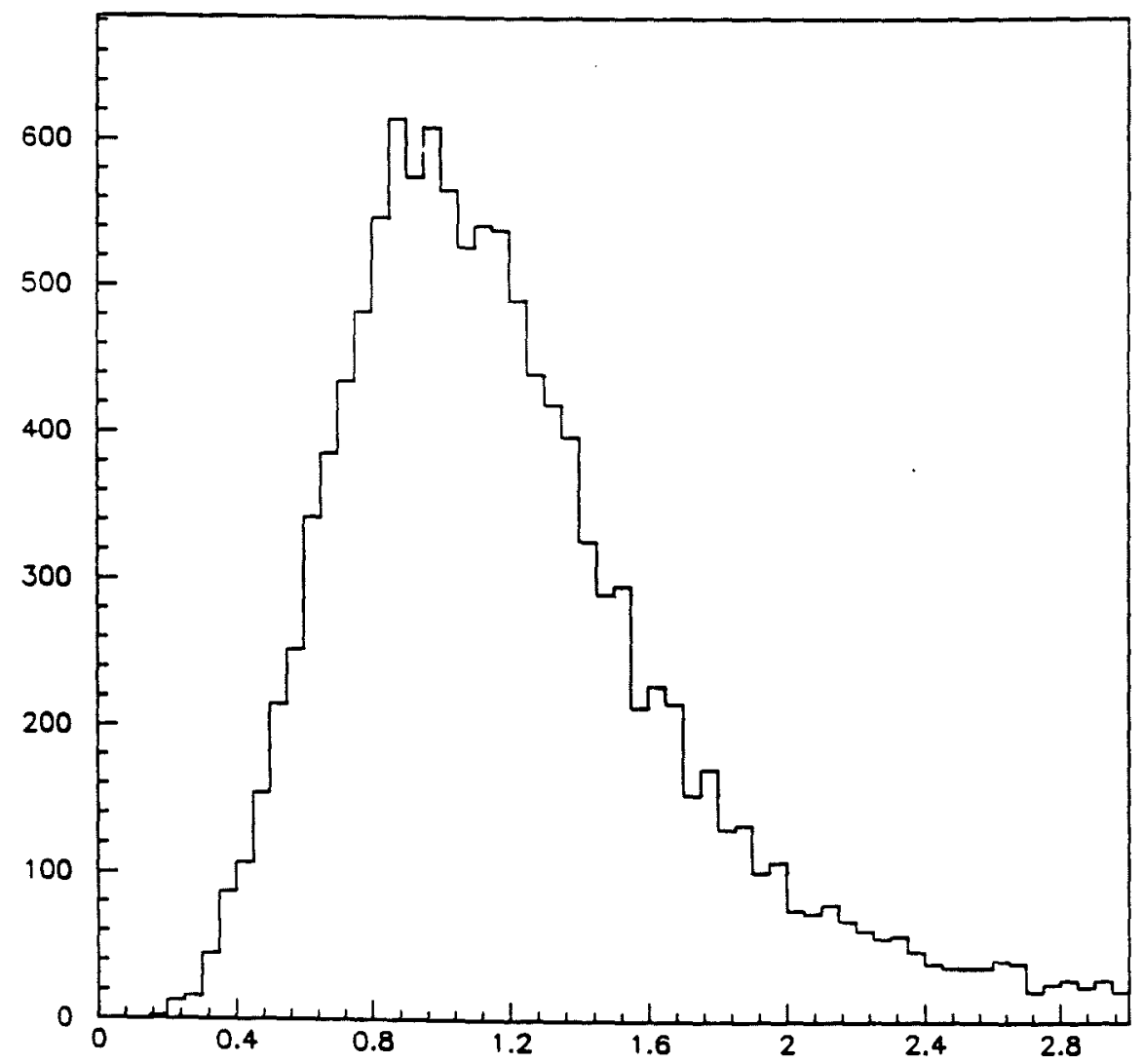

Figure 5.2: $\chi^{2}$ for 16 hit tracks 


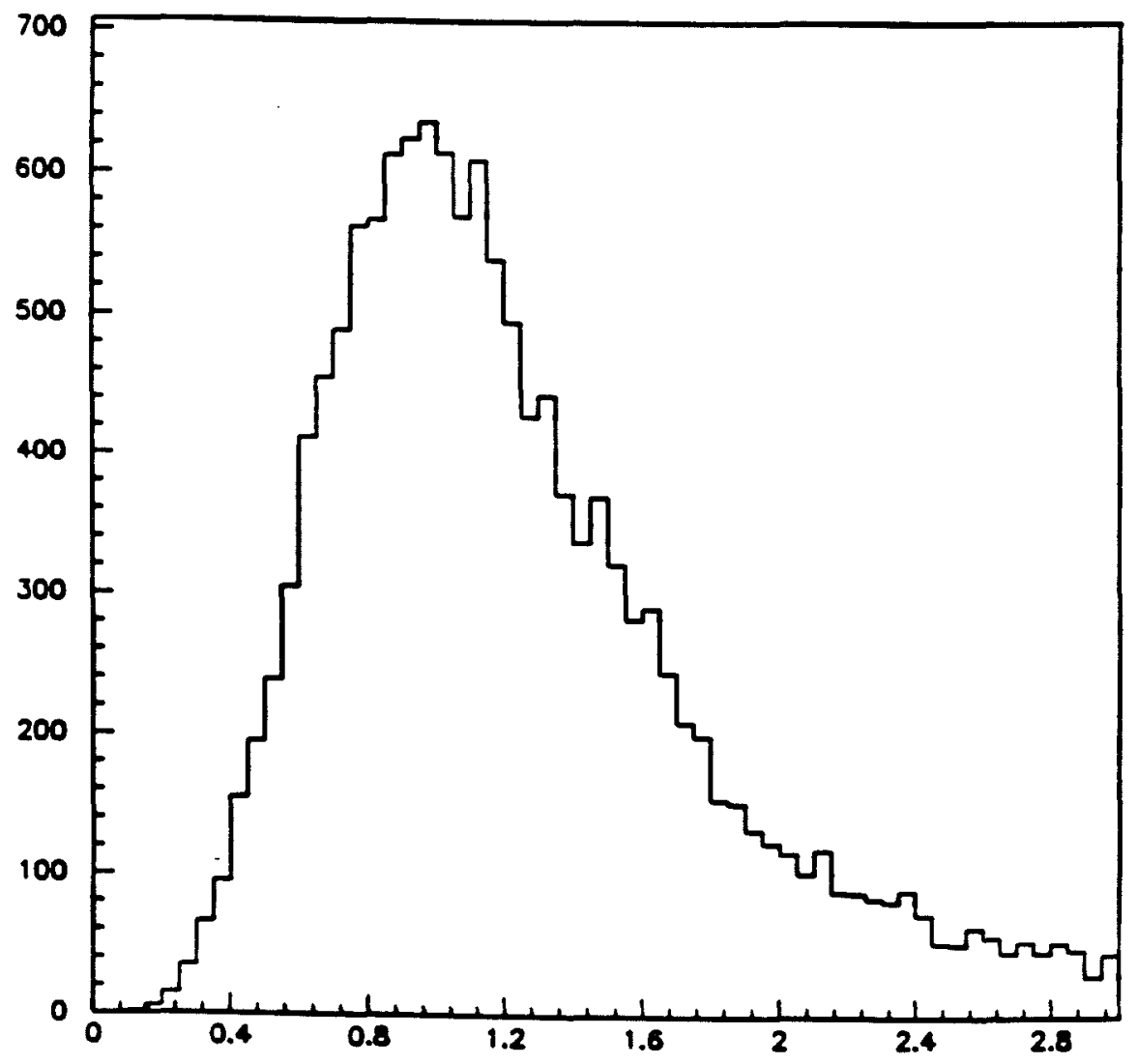

Figure 5.3: $\chi^{2}$ for 15 hit tracks 


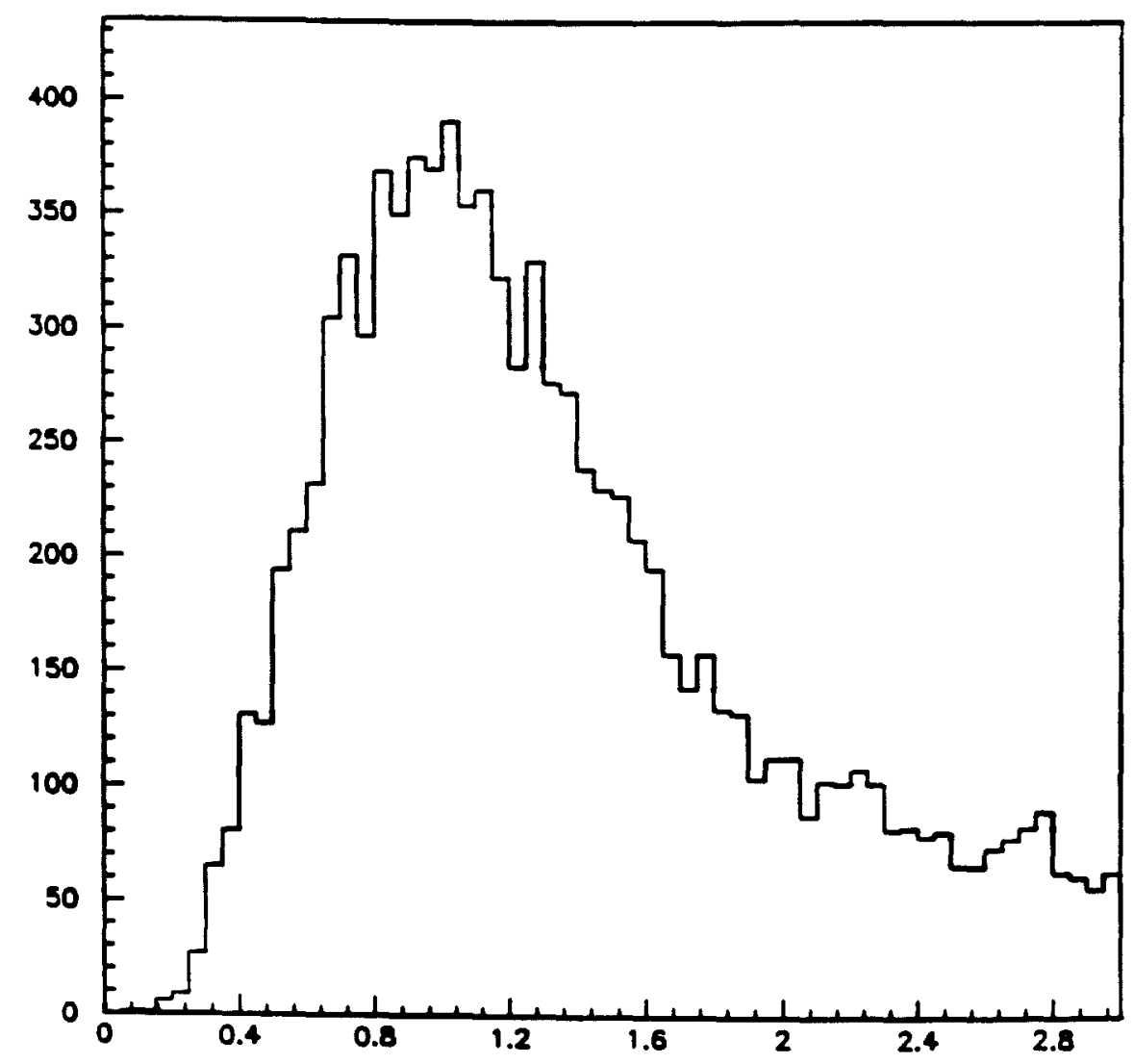

Figure 5.4: $\chi^{2}$ for 14 hit tracks 


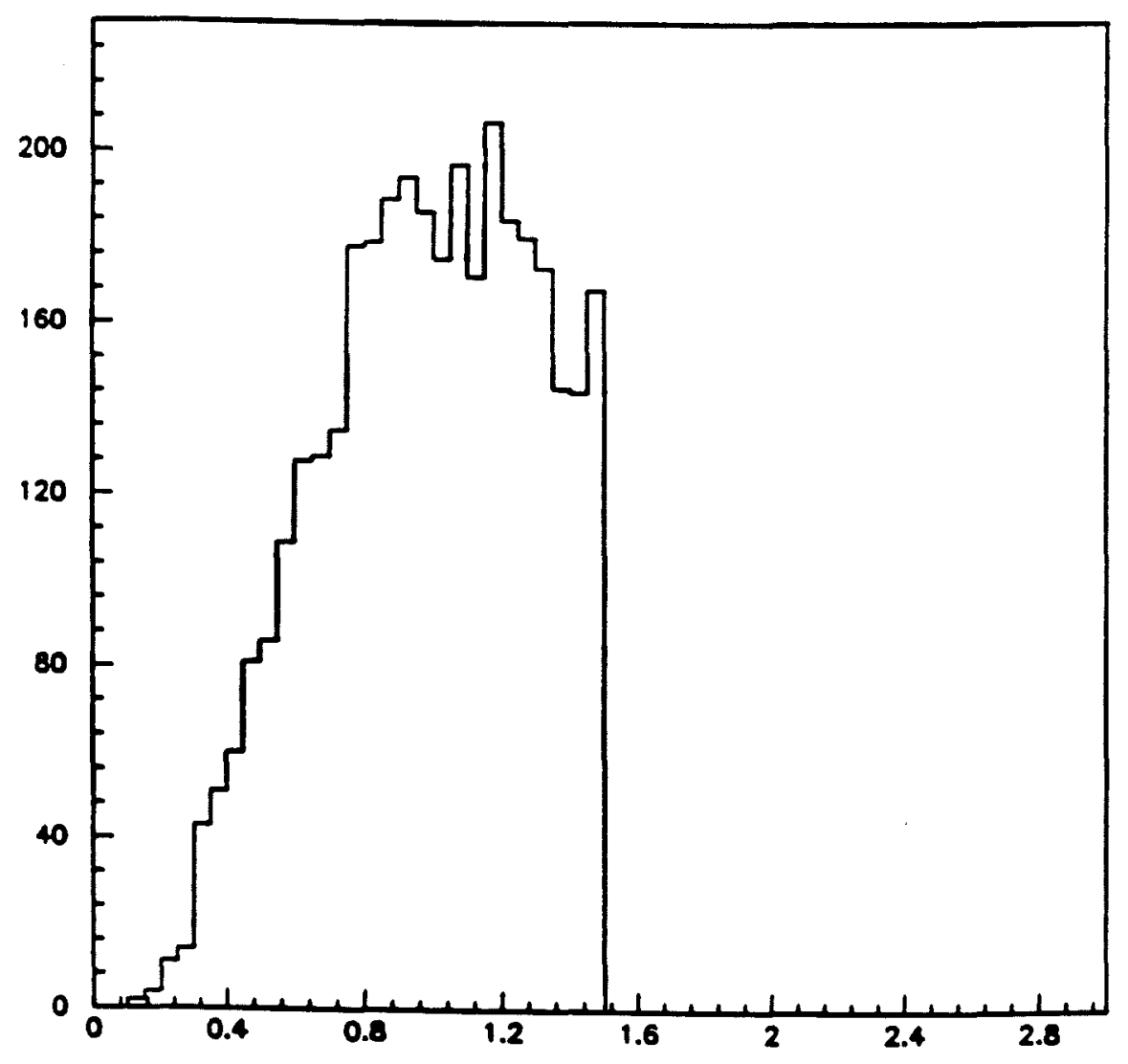

Figure 5.5: $\chi^{2}$ for 13 hit tracks 


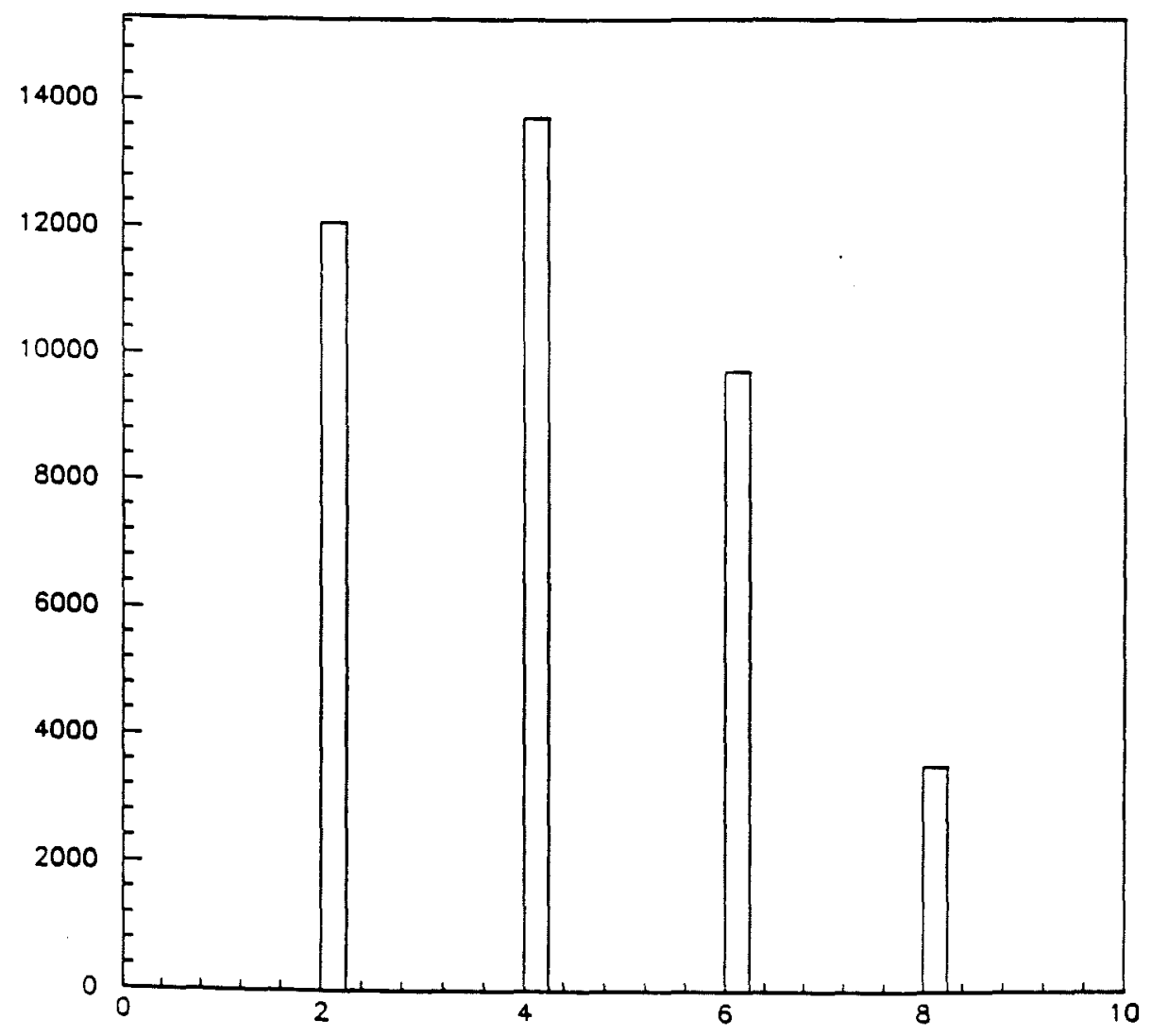

Figure 5.6: Relative Multiplicity of 16, 15, 14, 13 hit tracks 


\subsubsection{Beam tracking}

The beam chambers have three planes in each of the two orthogonal ( $\mathrm{X}, \mathrm{Y}$ ) views. Initially all the three hit tracks are found in each view. This is done by looping over all the hits in each pair of planes and searching for a hit in the third plane within 1.5 wirespacing of the road position. Once such a hit is found, a least-square fit is made to these three points. Then from the remaining hits which were not used in the three hit tracks, two hit view tracks are made. It may be noted that space tracks cannot be formed from orthogonal views.

\subsubsection{Linking at the magnet}

\section{Thin lens approximation}

To a first order approximation, the magnetic field can be assumed to be that of a dipole. The field lines are perpendicular to the $\mathrm{XZ}$ plane. The charged particles entering this dipole field will follow a circular path. The tracks observed in the SSD and MWPC can be considered as tangents to this circle. The path of the track through the magnet is in a symmetric fashion such that these tangents meet at the center plane of the magnet. In such a situation the bending of the tracks in the field can be considered as a sudden bend at this center plane of the magnet. The location of this plane is called the "effective center" of the magnet. The real magnet can be modelled as a dipole having an "effective length" $\left(L_{0}\right)$ centered at the effective center of the magnet. The deviations of the magnetic field from 
this approximation are parameterized and corrected for in reference [15:. The details of this parameterization are presented in Appendix A.

\section{Corrections to the effective field approximation}

Here the subscripts $\mathrm{x}$ and $\mathrm{y}$ refer to which view is used. There is a point $Z_{x}$ where the upstream $\mathrm{X}$ track intersects the $\mathrm{X}$ projection of the downstream space track. There is also a point $Z_{y}$ where the same happens to the $\mathrm{Y}$ track. $Z_{x}=Z_{y}$ only for infinitely high momenta. In such a situation:

$$
Z_{x}=Z_{y}=\frac{\int z B_{y} d z}{\int B_{y} d z}=Z_{0}
$$

For finite momenta of the tracks and finite $Y$ slopes the intercepts at $Z_{x}$ and $Z_{y}$ are not the same. Figure 5.7 and Figure 5.8 illustrate the angles the tracks make with the various directions.

For the tracks which do not travel symmetrically through the magnet, the magnitude of the angle of entrance to the magnetic field and the magnitude of the angle of exit from the magnetic field are not the same. In the $\mathrm{X}$ view the difference in the projection of these tracks to the center plane of the magnetic field along $\mathrm{Z}$ direction is:

$$
\delta_{1 x}=\frac{-L_{0}}{8}(\alpha+\beta)(\alpha-\beta)^{2}
$$

The $\mathrm{Z}$ component of the magnetic field also produces a change in the $\mathrm{X}$ slope of the track. The contribution from this to the projection difference at the center of the magnetic field in the $\mathrm{Z}$ direction is:

$$
\delta_{2 x}=\frac{-L_{0}}{2}(\sin \alpha-\sin \beta) \sin ^{2} \eta
$$




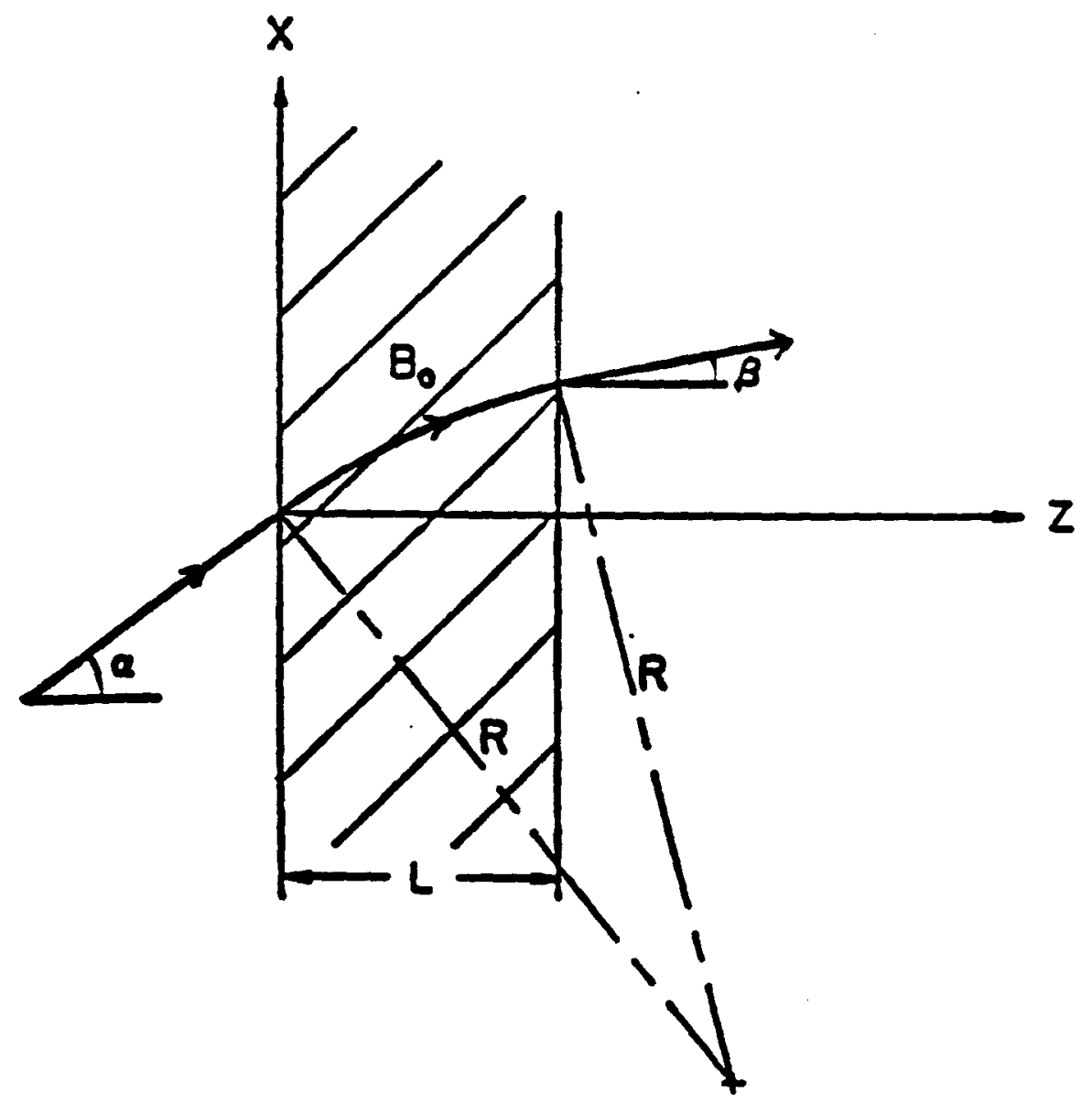

Figure 5.7: A track in the magnetic field 


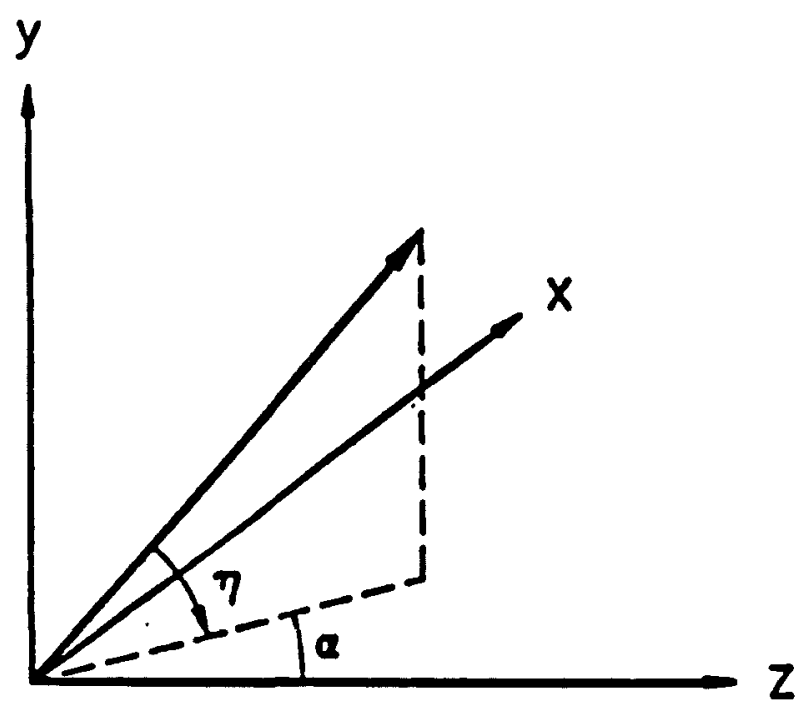

Figure 5.8: Angles involved in magnetic field corrections 
Hence the total projection difference in the $\mathrm{X}$ view is given by the following equation:

$$
\delta_{x}=\delta_{1 x}+\delta_{2 x}
$$

The difference in the slopes of the track in the $\mathrm{Y}$ view at the entrance and exit of the magnet is given by:

$$
\delta_{s 1 y}=\frac{\tan \eta}{2}\left(\sin ^{2} \beta-\sin ^{2} \alpha\right)
$$

The change in the slope of the track in the $\mathrm{Y}$ view due to the $\mathrm{Z}$ component of the magnetic field is given by:

$$
\delta_{s 2 y}=\eta(\alpha-\beta)\left[\beta-\frac{A_{0}}{L_{0}}(\alpha-\beta)\right]
$$

where:

$$
A_{0}=Z_{0}-\frac{L_{0}}{2}
$$

Hence the total change in the slope of the track in the $\mathrm{Y}$ view is given by:

$$
\delta_{s y}=\delta_{s 1 y}+\delta_{s 2 y}
$$

These quantities give rise to a change in the projection difference in the $\mathrm{Y}$ view for the track at the center plane of the magnet in the $\mathrm{Z}$ direction. They are given by:

$$
\begin{gathered}
\delta_{1 y}=\frac{L_{0}}{6}\left(\alpha^{2}+\alpha \beta+\beta^{2}\right) \tan \eta \\
\delta_{2 y}=-\frac{\tan \eta}{2}(\sin \alpha-\sin \beta)\left[A_{0}(\tan \alpha+\tan \beta)-L_{0} \tan \beta\right]
\end{gathered}
$$


The change in the slope of the track in the $\mathrm{X}$ view also produces a change in the slope in the $\mathrm{Y}$ view of the track. The contribution to the projection difference because of this effect is given by:

$$
\delta_{3 y}=\frac{-L_{0}}{4}\left(\alpha^{2}+\beta^{2}\right) \tan \eta
$$

Hence the total projection difference in the $\mathrm{Y}$ view is:

$$
\delta_{y}=\delta_{1 y}+\delta_{2 y}+\delta_{3 y}
$$

In Figure 5.9, Figure 5.10 and Figure 5.11 these quantities versus their expected values from simulated data are shown. Based on this figures, these quantities can be considered as the corrections one applies before testing whether a pair of tracks from SSD and MWPC links or not. This is described in the following section.

\section{Finding track links}

The MWPC tracks (space tracks) considered here are those matched in four different views. The SSD tracks are found only in two orthogonal views. Hence the MWPC tracks are less ambiguious than the SSD tracks. This fact has led to the following algorithm for linking. Each of the MWPC tracks is projected to the center of the magnet. For each MWPC track, all SSD tracks are projected to the center of the magnet. A MWPC and SSD track combination is called a "link pair." The absolute difference in projection of a link pair at the center of the magnet in a view is called the "projection difference." The absolute difference in the slopes of a link pair in a view is 


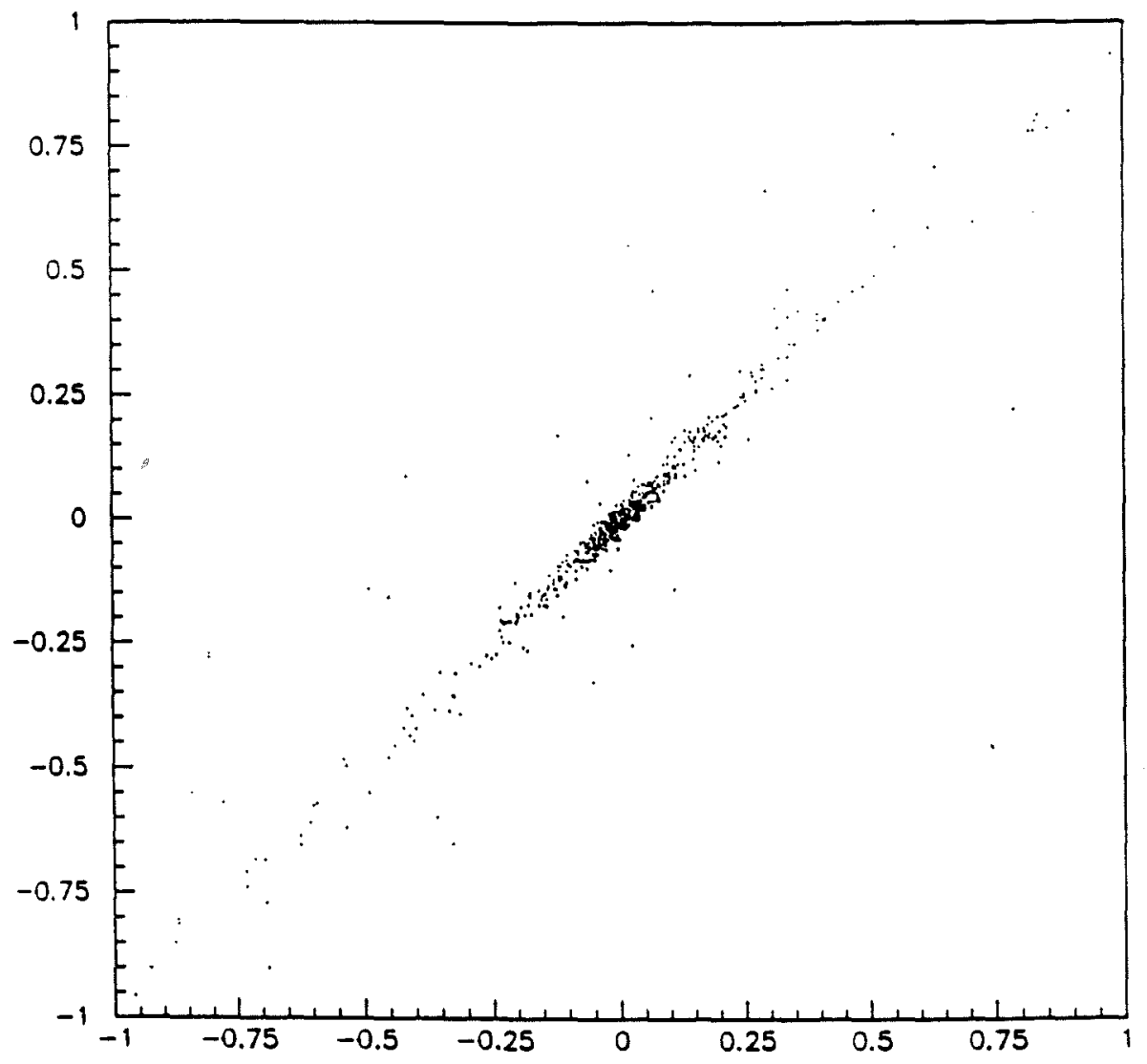

Figure 5.9: Field corrections in projections to magnet center versus their expected magnitudes in the $\mathrm{X}$ view 


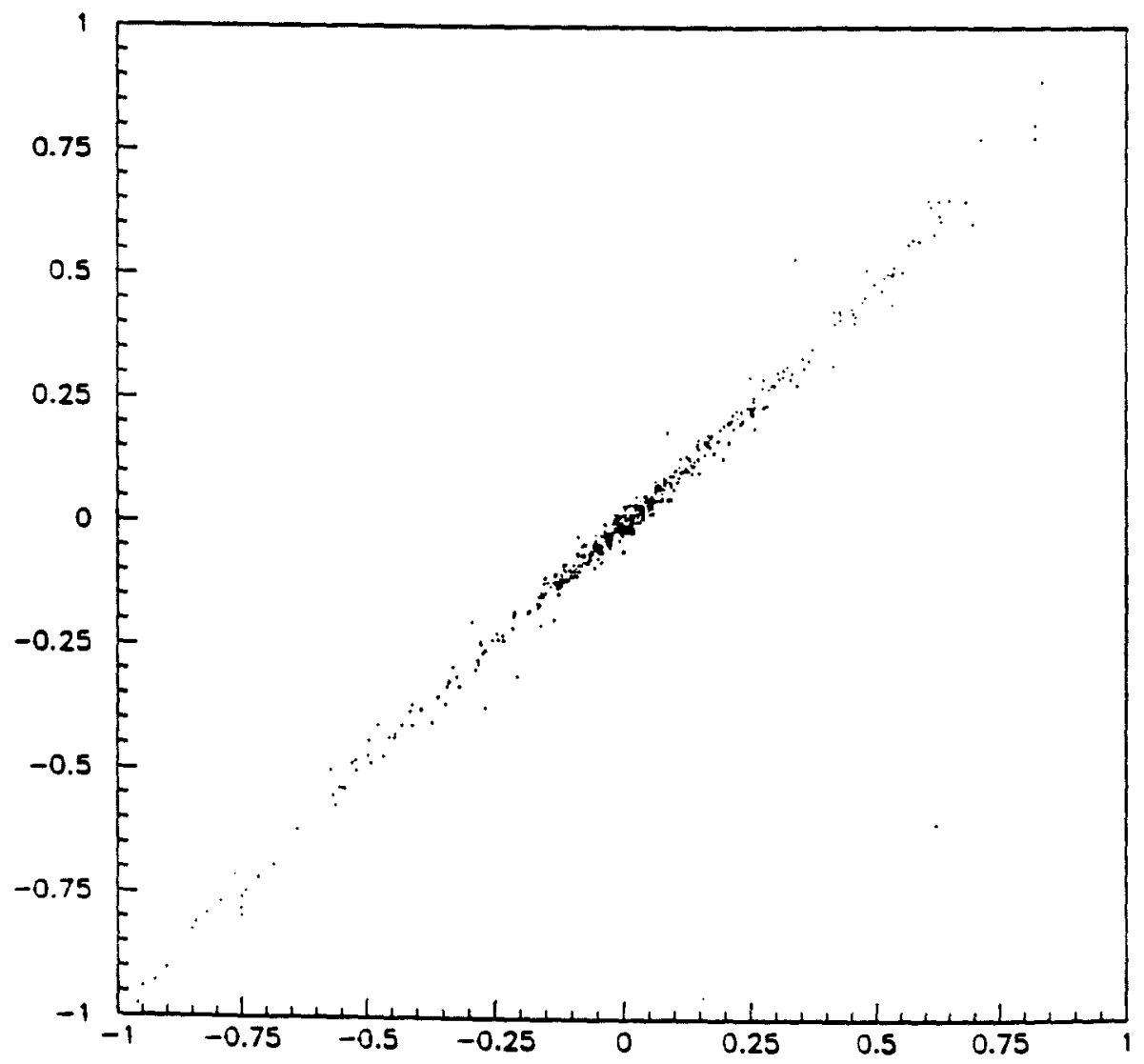

Figure 5.10: Field corrections in projections to magnet center versus their expected magnitudes in the $Y$ view 


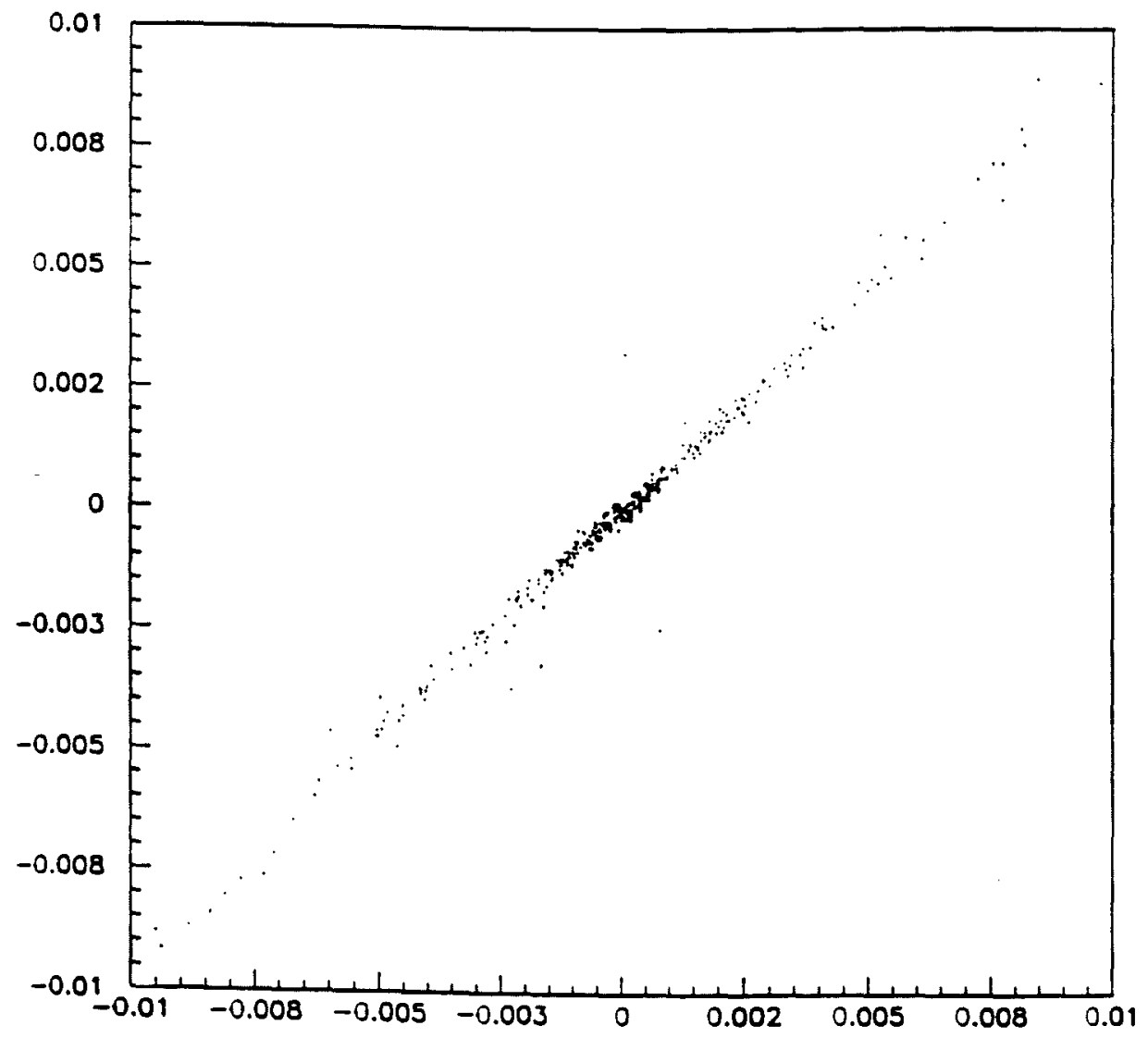

Figure 5.11: Field correction in $\mathrm{Y}$ view bend angle versus its expected magnitude 
called the "bend angle." These quantities are corrected for the non-uniform field effects discussed in the previous section. The main field of the magnet bends tracks in the $\mathrm{X}$ view. Hence the $\mathrm{XZ}$ plane is called the bending plane and the $\mathrm{YZ}$ plane is called the non-bending plane.

In the $\mathrm{X}$ view, if the tracks in a link pair were that of the same particle the corresponding projection difference should be close to zero for an ideal dipole field. If the projection difference is less than a cut value of $0.7 \mathrm{~cm}$, the link pair becomes what is called a "selected link pair." This cut value is determined by plotting the projection difference for several link pairs. All the selected link pairs are made into a list such that they are arranged in ascending order of the projection difference. The first one in this list is considered as the "space track best link" in the X view. The next four in this list are called "extra space track links." This procedure is repeated for all space tracks.

In the $\mathrm{Y}$ view if the tracks in a link pair were that of the same particle, the projection difference would be zero and the bend angle would be zero. A "link $\chi 2 "$ is defined, which is a weighted sum of the squares of the projection difference and the bend angle. If this quantity is less than a cut value of $2.5 \mathrm{mrad}$, then the link pair becomes a selected link pair. This cut value is determined by plotting projection differences and bend angles for several link pairs. From all the selected link pairs the space track best link in the $\mathrm{Y}$ view and the extra space track links are found using the same procedure as that in the $\mathrm{X}$ view. 


\section{Performance of linking}

In Figure 5.12 and Figure 5.13 the projection differences of the linked tracks from the MWPC's and the SSD's at the center of the magnet are shown. In Figure 5.14 the difference in the $Y$ slope of the linked tracks from the SSD and MWPC are shown. These figures illustrate the amount of resolution one can get in the linking procedure. For the $\mathrm{X}$ view and $\mathrm{Y}$ view the resolution in the projection difference of the tracks is $2 \mathrm{~mm}$. In the $\mathrm{Y}$ view the resolution in the bend angle is $0.8 \mathrm{mrad}$.

\subsubsection{Philosophy of vertex reconstruction}

In this program the location of the primary vertex is determined using the tracks reconstructed in the two-view SSD system. The vertices are found in each of the two views first and then they are matched to get the threedimensional location of the vertex [31].

\section{View vertex finding}

If there are more than four tracks identified as 4-hit tracks and linked with MWPC tracks through the magnet, then only those tracks are used for view vertex reconstruction. Otherwise, if there are more than three 4-hit tracks, all the 4 -hit tracks are used for vertex reconstruction. If there are are only three or less 4-hit tracks, then all the 3-hit tracks which are linked with MWPC tracks are used for vertex reconstruction.

The selected tracks have to be within the lateral dimensions $(2 \mathrm{~cm}$ by 


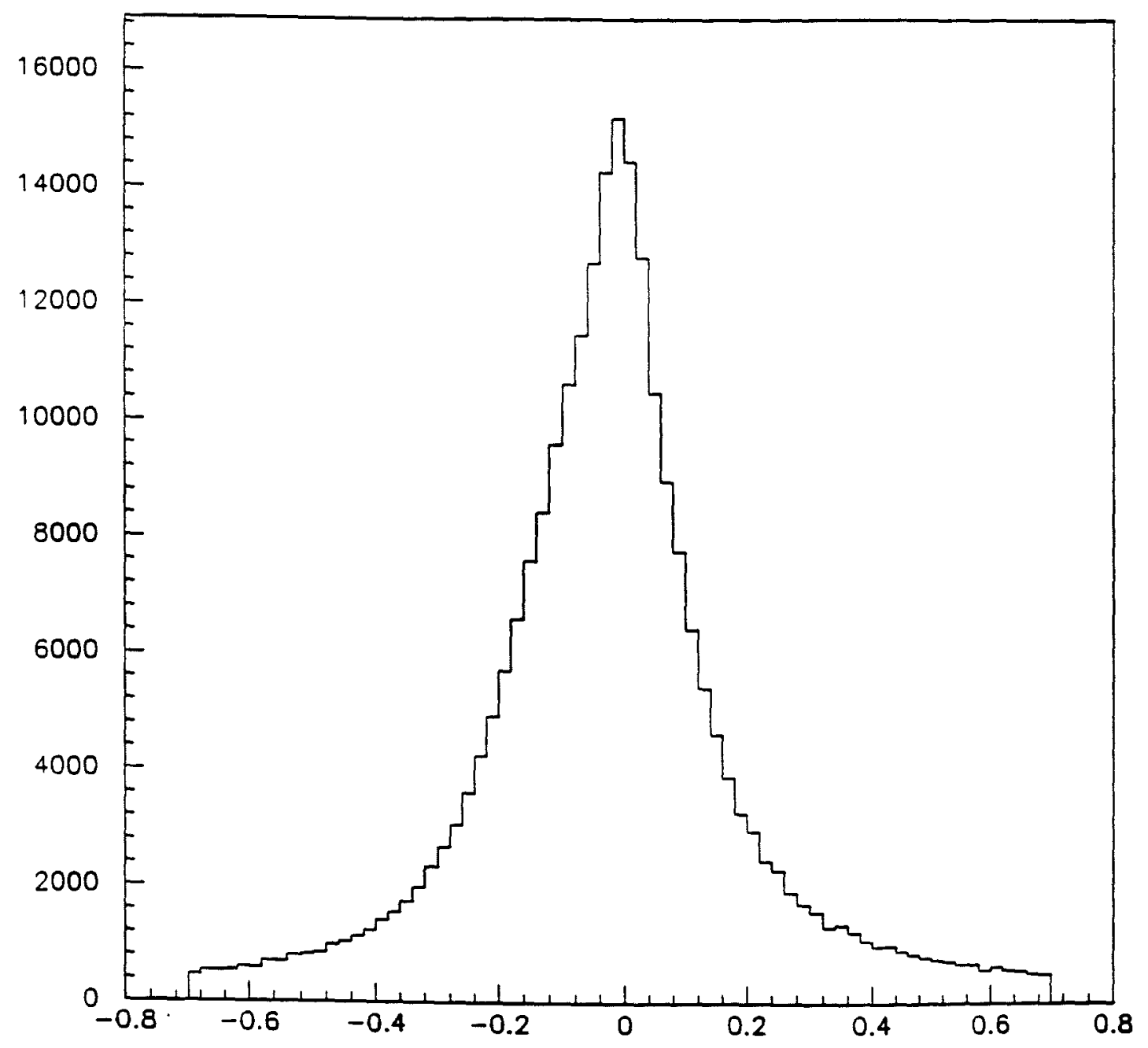

Figure 5.12: $\Delta x$ at the magnet center 


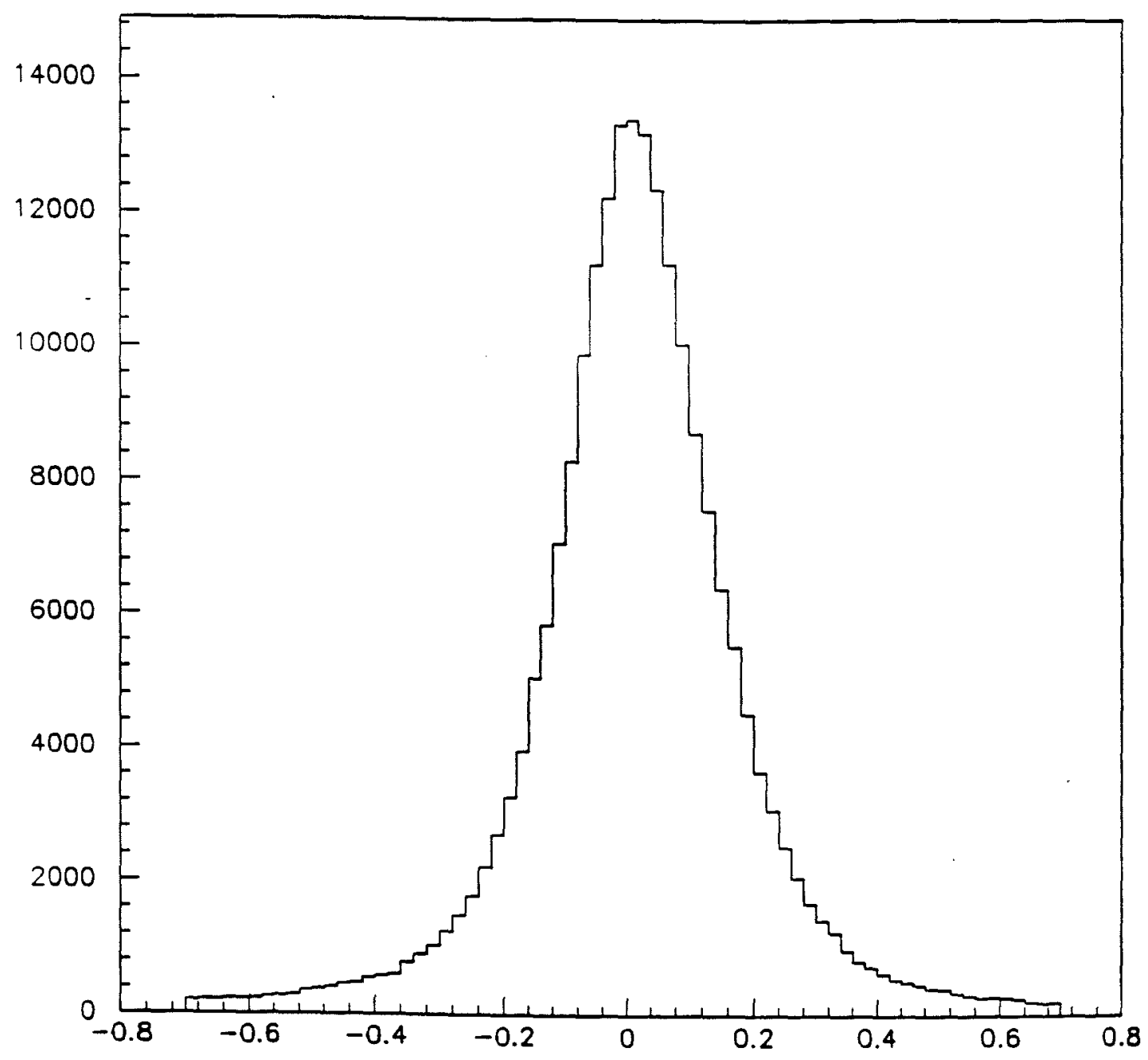

Figure 5.13: $\Delta y$ at the magnet center 


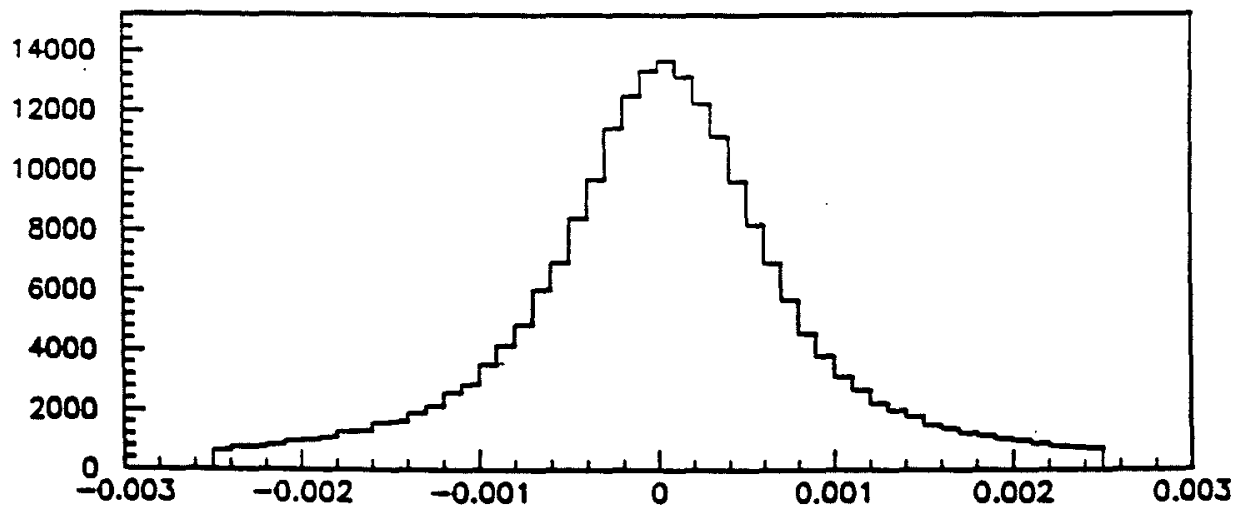

Figure 5.14: $\Delta$ slope y at the magnet center 
$2 \mathrm{~cm}$ ) of the target when projected to the target region. If there are not enough tracks selected, the vertex reconstruction is not performed.

The view vertex finding procedure is the same in both the $\mathrm{X}$ and $\mathrm{Y}^{\mathrm{r}}$ views. In the $X$ view, the $\chi^{2}$ of a track which goes through points $\left(x_{j}, z j\right)$ in each of the SSD $\mathrm{X}$ view planes and the primary vertex is:

$$
\chi_{1}^{2}\left(a_{k}, b_{k}\right)=\sum_{j} \frac{\left[x_{j}-\left(a_{k} z_{j}+b_{k}\right)\right]^{2}}{\sigma_{j}^{2}}
$$

where: $a_{k}=$ slope of the track

$b_{k}=$ intercept of the track

$k=$ track number

$\sigma_{j}=$ track projection at the vertex.

$\mathrm{j}=$ plane number

Here the summation is over all the planes used in reconstructing the track.

For tracks which do not pass through the primary vertex, the $\chi^{2}$ is:

$$
\chi_{2}^{2}\left(a_{k}^{\prime}, b_{k}^{\prime}\right)=\sum_{j} \frac{\left[x_{j}-\left(a_{k}^{\prime} z_{j}+b_{k}^{\prime}\right]^{2}\right.}{\sigma_{j}^{2}}+\frac{\left[X_{0}-\left(a_{k}^{\prime} Z_{0}+b_{k}^{\prime}\right)\right]^{2}}{\sigma_{0}^{2}}
$$

where:

$a^{\prime}=$ slope of the track

$b^{\prime}=$ intercept of the track

$k=$ track number

$Z_{0}=\mathrm{Z}$ location of the vertex

$X_{0}=\mathrm{X}$ location of the vertex

$\sigma_{0}=$ track projection at the vertex

$$
\chi_{\text {vertex }}^{2}=\chi_{2}^{2}-\chi_{1}^{2}
$$


The impact parameter for a track is defined as the normal distance from the track to the candidate position of the vertex. In Figure 5.15, Figure 5.16 and Figure 5.17 these quantities are plotted for tracks in the two views of the SSD's and for tracks in the Y view in the MWPC's. The width of these distributions illustrates the resolution one can get from each of these systems at the vertex location. For MWPC tracks this resolution is $0.5 \mathrm{~cm}$ and for SSD tracks it is $0.008 \mathrm{~cm}$.

Minimizing this $\chi^{2}$, one can solve for the vertex position. This is an iterative fitting procedure. At the end of each iteration the track with the largest impact parameter is eliminated from being included as a selected track for the next iteration. This is continued until either the average impact parameter of all the tracks associated with the vertex is less than $20 \mu \mathrm{m}$ or the largest impact parameter on an individual track is less than $50 \mu \mathrm{m}$ or one is left with only two tracks. At the end of each iteration the vertex position is redetermined. This procedure is repeated for both the $\mathrm{X}$ view and $\mathrm{Y}$ view. The algorithm is optimized to find the best primary vertex in each view.

\section{Matched vertex finding}

The view vertex in one view is refitted using the tracks in that view and the $\mathrm{Z}$ location of the vertex as determined in the other view as a starting point. The same procedure is repeated in the other view. A combination of two vertices with one from each view, is called a vertex pair. There are four such vertex pairs in this scheme. A matched vertex is a location in 


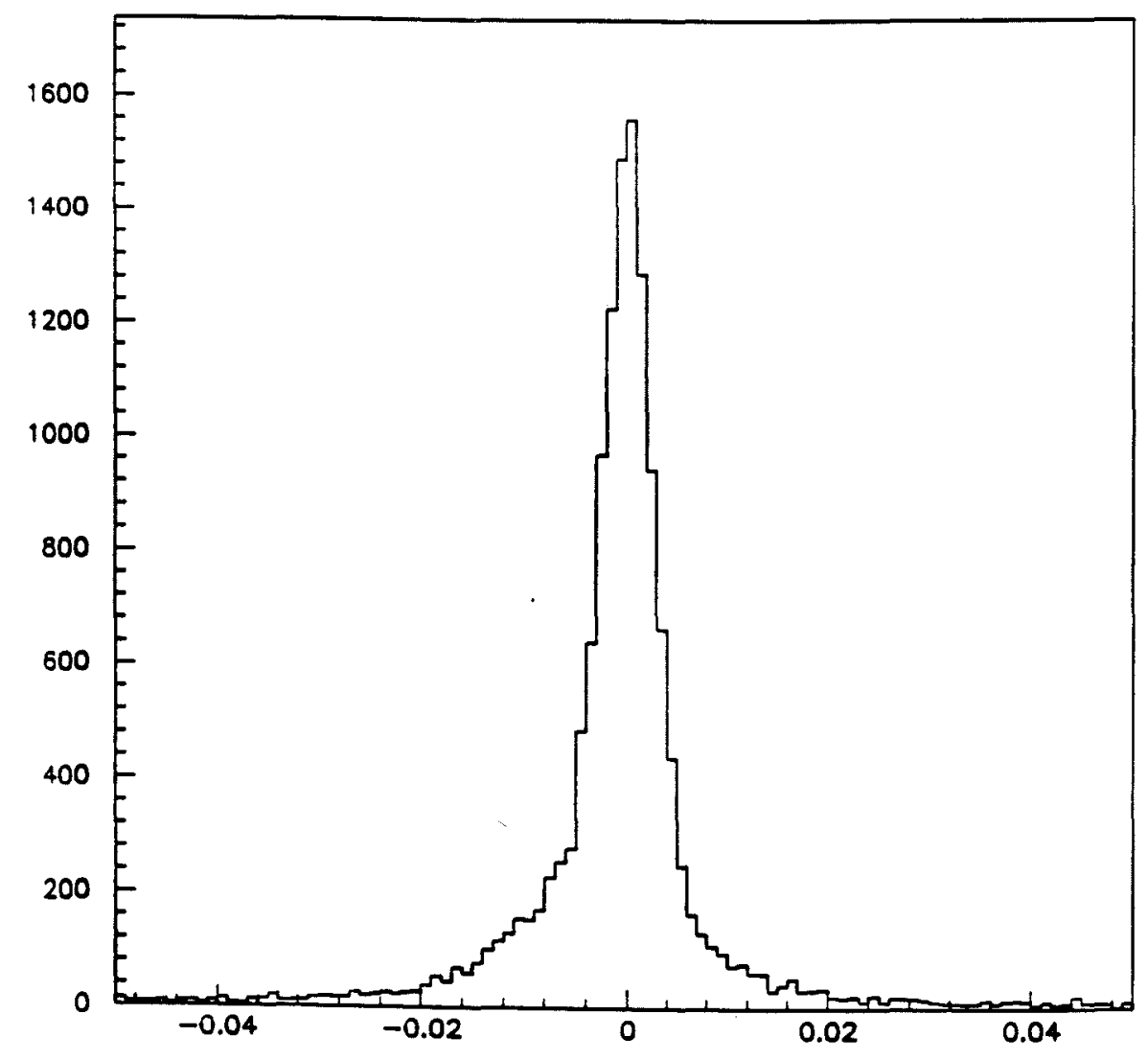

Figure 5.15: Impact parameter distributions for SSD tracks in the $\mathrm{X}$ view 


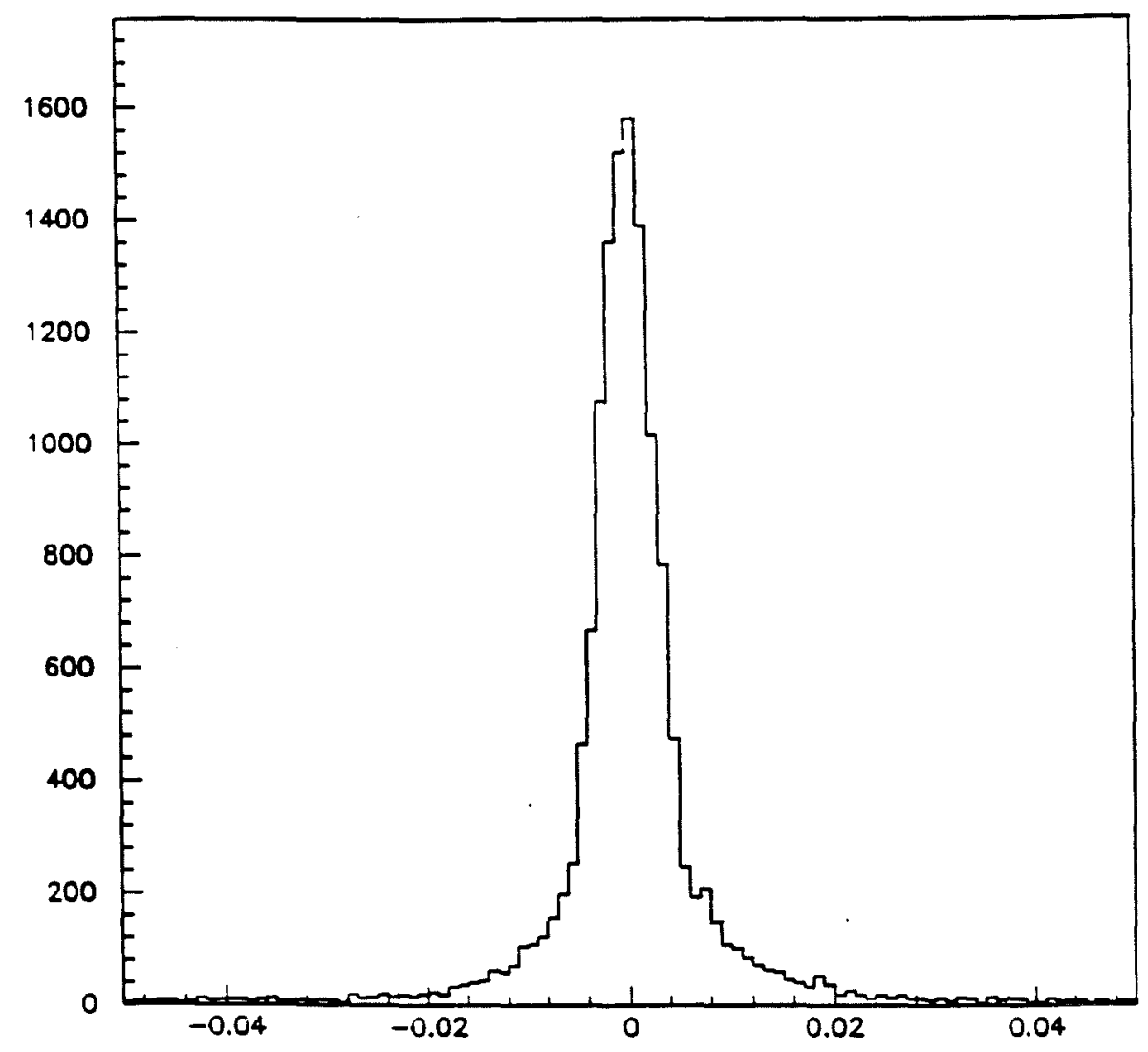

Figure 5.16: Impact parameter distributions for SSD tracks in the $\mathrm{Y}$ view 


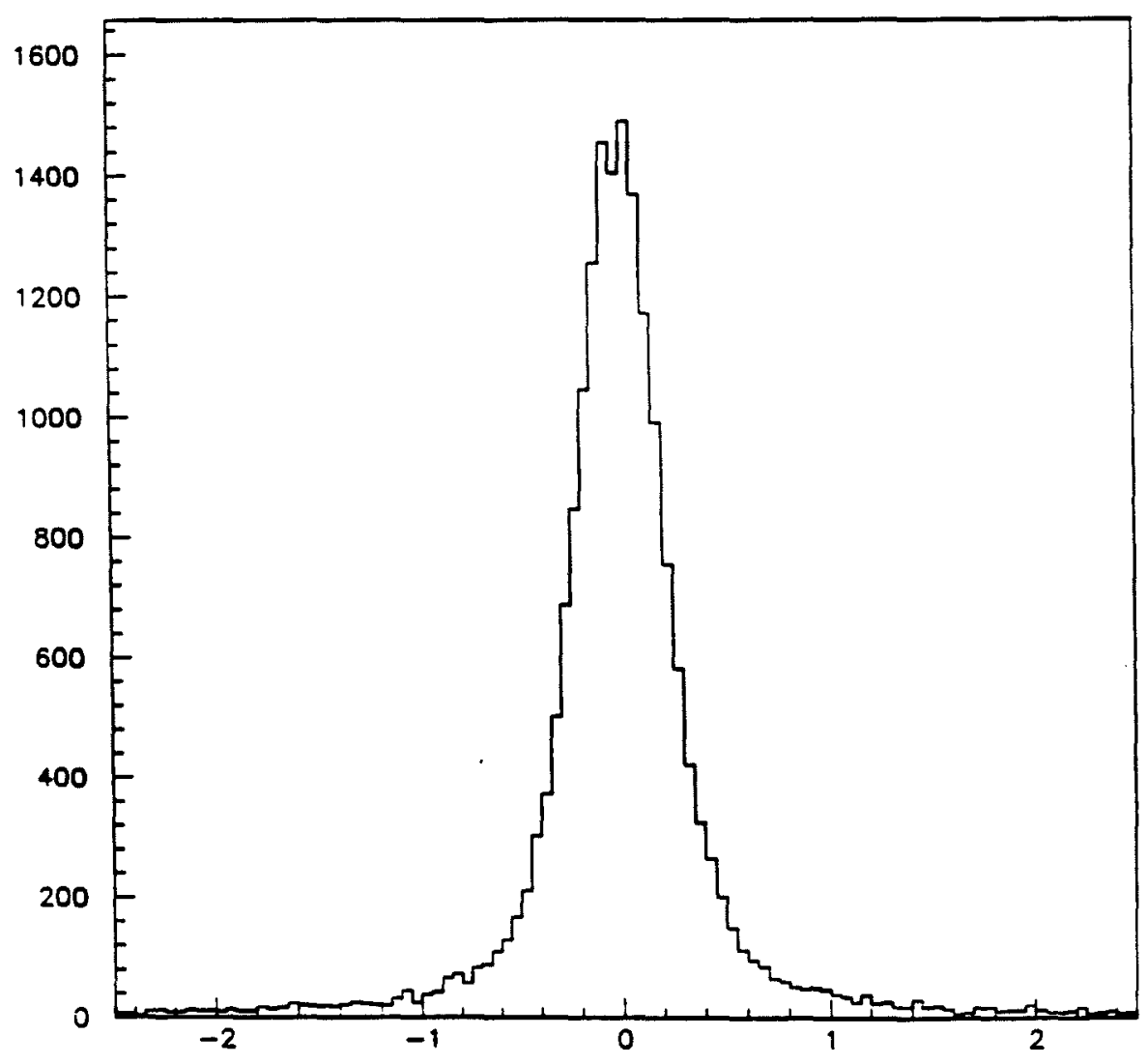

Figure 5.17: Impact parameter distribution for MWPC tracks in the $\mathrm{Y}$ view 
space where the best match of view vertices from the two views is obtained. The vertex pair for which the difference in $\mathrm{Z}$ location of the two vertices is least, is considered as the matched vertex. The $\mathrm{Z}$ coordinate of the matched vertex is the weighted average of the $\mathrm{Z}$-coordinates of the two view vertices, where the weight of each view vertex is the reciprocal of the error in the $\mathrm{Z}$-coordinate of each view vertex as determined by the $\chi^{2}$ minimization procedure.

\section{Vertex resolution}

The vertex resolution along the beam direction is about $600 \mu \mathrm{m}$ and along the transverse direction it is about $20 \mu \mathrm{m}$. Figure 5.18 and Figure 5.19 show the result of the vertex reconstruction. In Figure 5.18 the $\mathrm{Z}$ distribution of vertices with and without the target are shown. This figure illustrates the vertex finding capability of the system. In Figure 5.19 the difference in the $\mathrm{Z}$ coordinate of the vertices from the two views of the SSD's are plotted. The width of this distribution is a measure of the vertex resolution of the system.

\subsubsection{Relinking}

In this procedure, if a track seems to come from the vertex region in the non-bending plane and if the space track best link does not come from the

vertex region and some extra links come from the vertex, a "relink $\chi^{2}$ " is formed. This quantity is the weighted sum of the squares of the impact 

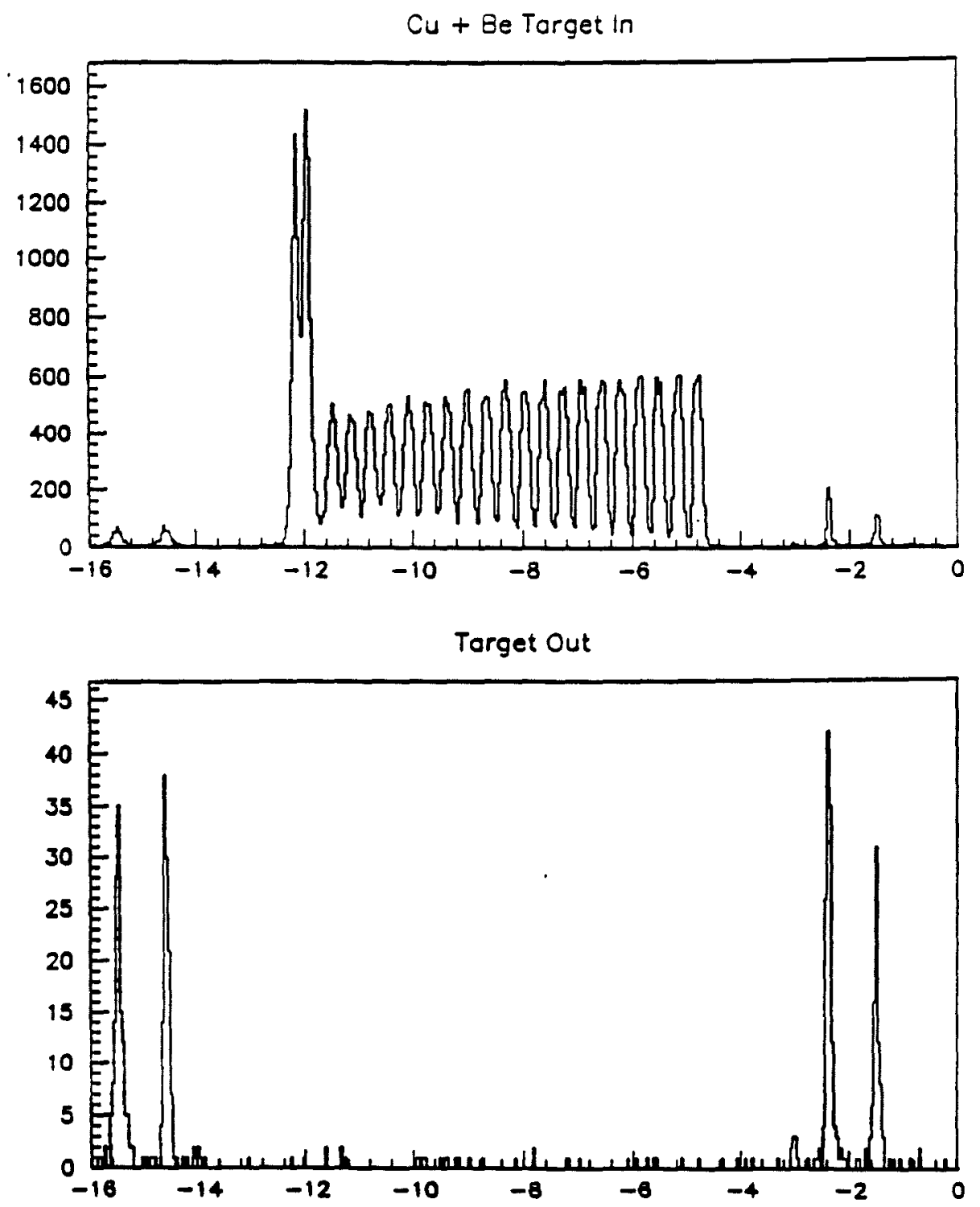

Figure 5.18: $\mathrm{Z}$ coordinate of the matched vertices 


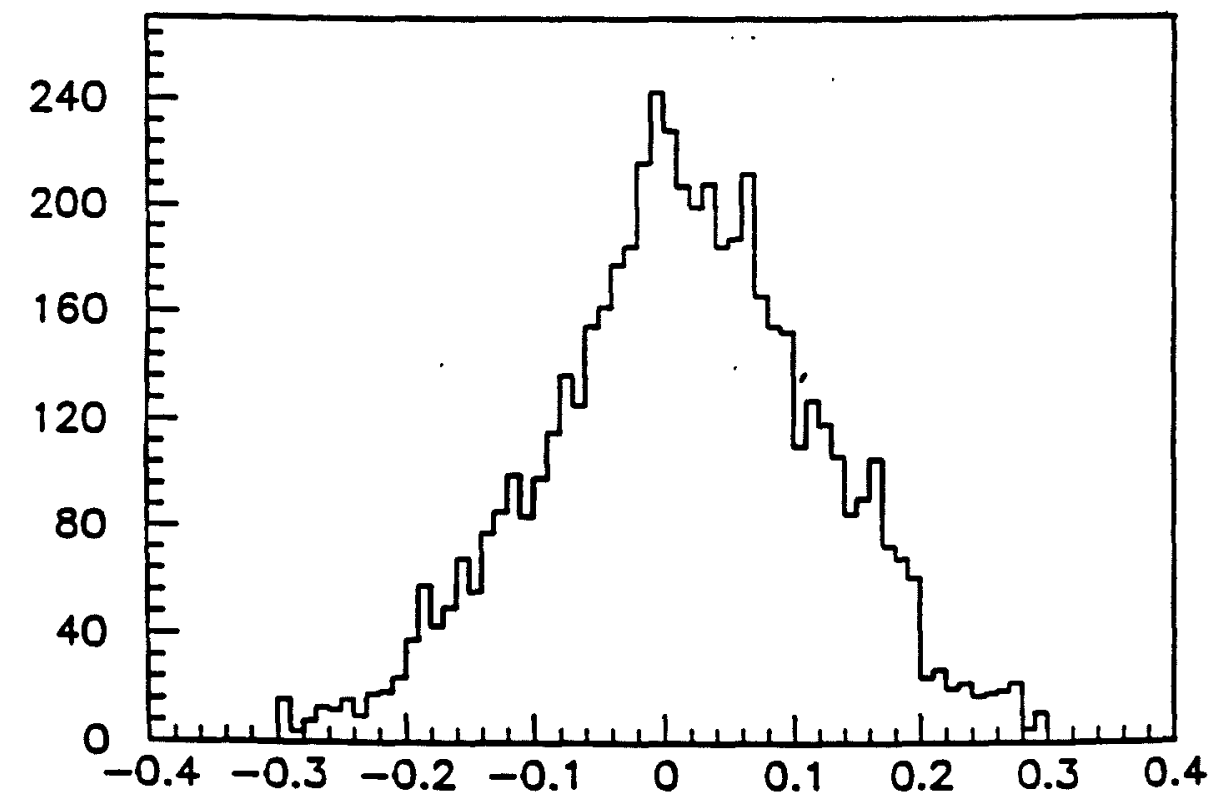

Figure 5.19: $V_{z}$ difference in the two views 
parameter and the projection difference. This is calculated for the first five selected link pairs. The "physics best link" is that selected link pair for

which the relink $\chi^{2}$ is the minimum. The other four selected link pairs are considered "physics extra links." This procedure is done for all space tracks.

If the upstream link does not exist for a space track in the $\mathrm{X}$ view, a line joining the intersection of the track at the center of the magnet and the vertex is assumed to be the $\mathrm{X}$ view upstream track.

If no vertex is found in the event, the center of the target system is considered as the nominal vertex location. If there is no upstream link in the $\mathrm{Y}$ view for a space track, the projection of this downstream track to the target region is considered as the $\mathrm{Y}$ view upstream track.

\section{Results of vertex reconstruction}

About $95 \%$ of the events collected had a vertex. The events in which a vertex could not be determined were mostly very high multiplicity events or those from multiple interactions of different beam particles in the target.

\subsubsection{Determination of momentum and charge}

\section{Modification of $p_{T}$ kick using real data}

During the data taking of E706, experiment E672 has used a dimuon trigger. While analysing data, some dimuon triggered events were identified and the momenta of these dimuons were calculated using the E706 tracking system. A mass plot of the oppositely charged dimuons obtained in this way show 
a peak near the mass of the $J / \psi$. A fine tuning of the value $B_{0}$ resulted in a slight modification of the $p_{T}$ kick of the magnet and the $J / \psi$ peak moved closer to its real value (see Figure 5.20). The modified value of $B_{0}$ is 6.4 kGauss.

\section{Momentum and charge calculation}

The formulae used for these calculations are as follows. Let $\theta_{1}$ and $\theta_{2}$ be the angles of the directions of a track with respect to the $\mathrm{Z}$ axis in the $\mathrm{XZ}$ plane, upstream and downstream of the magnet. Let $B_{0}$ be the magnitude of the effective magnetic field in the thin lens approximation described in Section 5.1.4.

Then the charge of the track is given by:

$$
q=\operatorname{sign}\left(\theta_{1}-\theta_{2}\right) \cdot \operatorname{sign}\left(B_{0}\right)
$$

Here the symbol sign refers to the sign of the quantity which follows the symbol.

$$
\begin{gathered}
p_{k i c k}=q B_{0} L_{0} \\
\sqrt{p_{x}^{2}+p_{z}^{2}}=p_{x z}=\frac{p_{k i c k}}{\sin \theta_{2}-\sin \theta_{1}} \\
\frac{p_{x}}{p_{z}}=\tan \theta_{x} \\
\frac{p_{y}}{p_{z}}=\tan \theta_{y}
\end{gathered}
$$

Solving the above equations, the three components of the momentum of the track are obtained. The range of momenta for which the tracking system is designed is as follows: when the momentum of the particle is below 


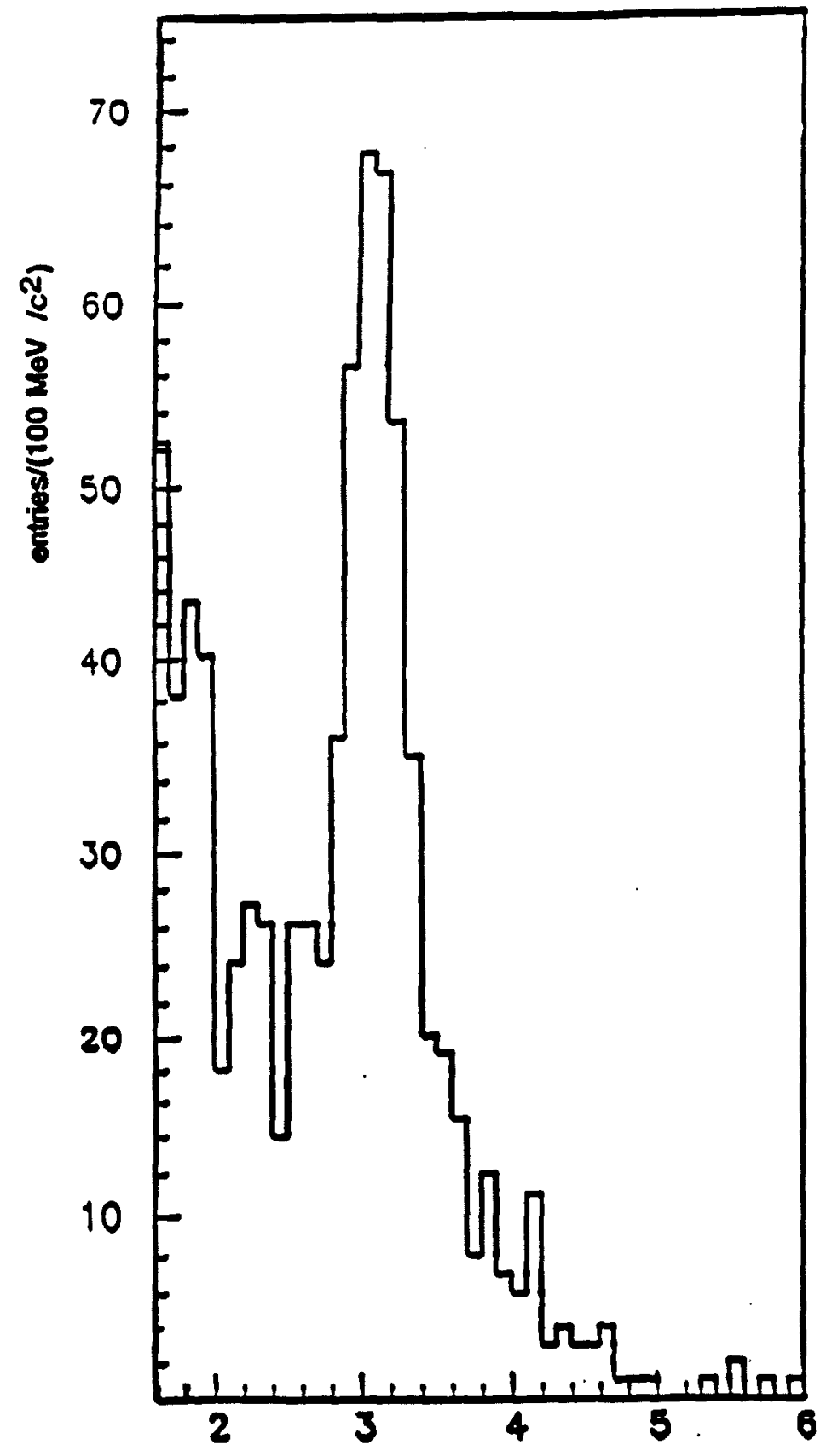

Figure 5.20: Dimuon $\left(\mu^{+} \mu^{-}\right)$mass in $\mathrm{GeV} / c^{2}$ 
$1 \mathrm{GeV} / \mathrm{c}$, it may curl up in the magnet and hence will not be detected in the MWPC's. The non-uniformity of the magnetic field may affect the momentum of the particles whose momenta are below $3.5 \mathrm{GeV} / \mathrm{c}$, if they happened to go through the edges of the magnet. This effect was found to be very small. When the momentum is above $150 \mathrm{GeV} / \mathrm{c}$, the relative error in the momentum is:

$$
\frac{\Delta P}{P}=\frac{0.1 P}{100.0}=0.15
$$

which is non-negligible. Hence any momentum measured above this value is likely to have a big error associated with it.

\subsection{Detector simulation}

The detector simulation is done using the CERN software package GEANT [9]. GEANT has the capability of setting up detector volumes of normal shapes. It also simulates the common physics processes the particles undergo while going through the various materials. This package was used to create fake data to test reconstruction results. It was used also to test the acceptance of the apparatus. The input data to the detector simulation was produced from the package ISAJET [26]. This has the capability of simulating the underlying physics in the direct photon events. 


\subsection{Efficiency of the tracking system}

The hardware efficiency of the tracking system is measured in the following manner. From the real data, the hits on the plane for which the efficiency is to be measured are suppressed and the reconstruction of the tracks which made a hit in every other plane is performed. This is to ensure that the tracks reconstructed are not fake tracks. These tracks are projected to the plane in which the efficiency is to be determined and a check of whether there was actually a hit in that plane near the projected position is performed. A distribution of the locations of projections of the tracks on the plane is made. Another distribution of the locations of the projection of the tracks on the plane is made when there is a hit near the projected position. The ratio of the second distribution to that of the first one gives a measure of the efficiency of that plane. This was done for all the planes. Figure 5.21 shows the efficiency of plane number 20. The dips this figure correspond to dead channels or regions where the MWPC was insensitive. Table 5.1 shows the measured efficiency of each of the planes of the MWPC. The typical efficiency was $95 \%$ for most of the planes.

In the central region of the MWPC's, the efficiency is lower since the cathode planes were set to be more insensitive in that region. The particle multiplicity is higher due to the fragments of the beam jet, and hence the chances of misidentification is high in those regions.

The efficiency of the reconstruction program is found by running the program on Monte Carlo generated data. Figure 5.22 shows the calculated 
Table 5.1: Efficiency of MWPC planes

\begin{tabular}{|c|c|}
\hline Plane number & Efficiency of the plane \\
\hline 1 & 0.92 \\
\hline 2 & 0.95 \\
\hline 3 & 0.94 \\
\hline 4 & 0.94 \\
\hline 5 & 0.95 \\
\hline 6 & 0.92 \\
\hline 7 & 0.94 \\
\hline 8 & 0.92 \\
\hline 9 & 0.93 \\
\hline 10 & 0.95 \\
\hline 11 & 0.94 \\
\hline 12 & 0.92 \\
\hline 13 & 0.95 \\
\hline 14 & 0.95 \\
\hline 15 & 0.94 \\
\hline 16 & 0.94 \\
\hline
\end{tabular}




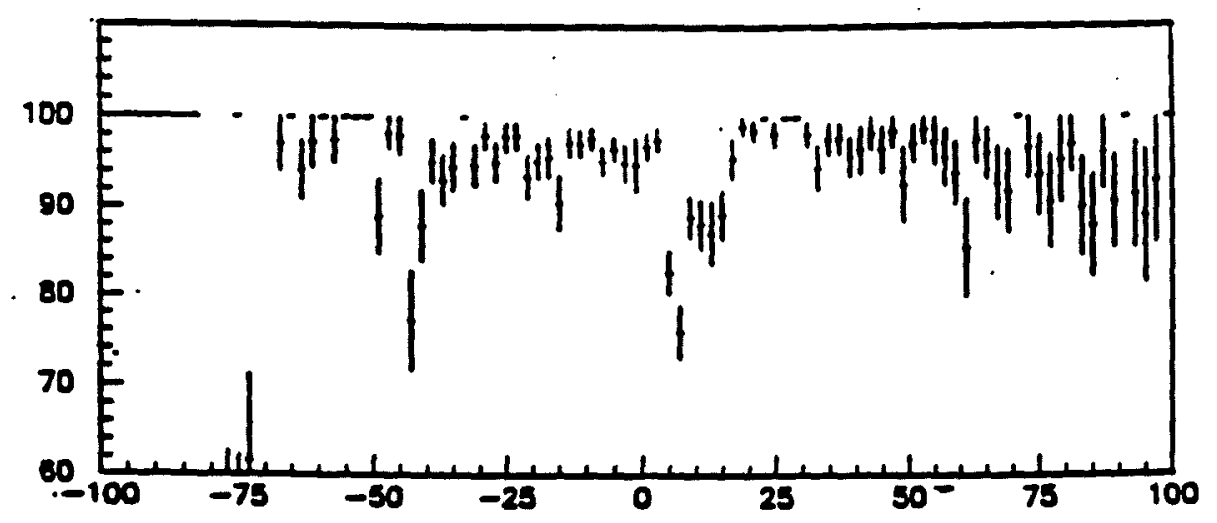

Figure 5.21: Efficiency of plane number 20 
versus generated momenta of the tracks. The reconstructed momentum seems to be in agreement with the generated momentum.

The efficiency of the linking of tracks is measured by plotting the fraction of tracks linked in each view. Figure 5.23 shows this. From this figure it can be seen that on the average about $85 \%$ of the tracks linked. The inefficiency in linking is mainly due to the high multiplicity of the tracks in the central region of the magnet. In Figure 5.24 the $\frac{\Delta P}{P}$ versus $\% \mathrm{P}$ is shown (here $P=$ Momentum of a track). The data can be parameterised as $0.1 \%$ P. 


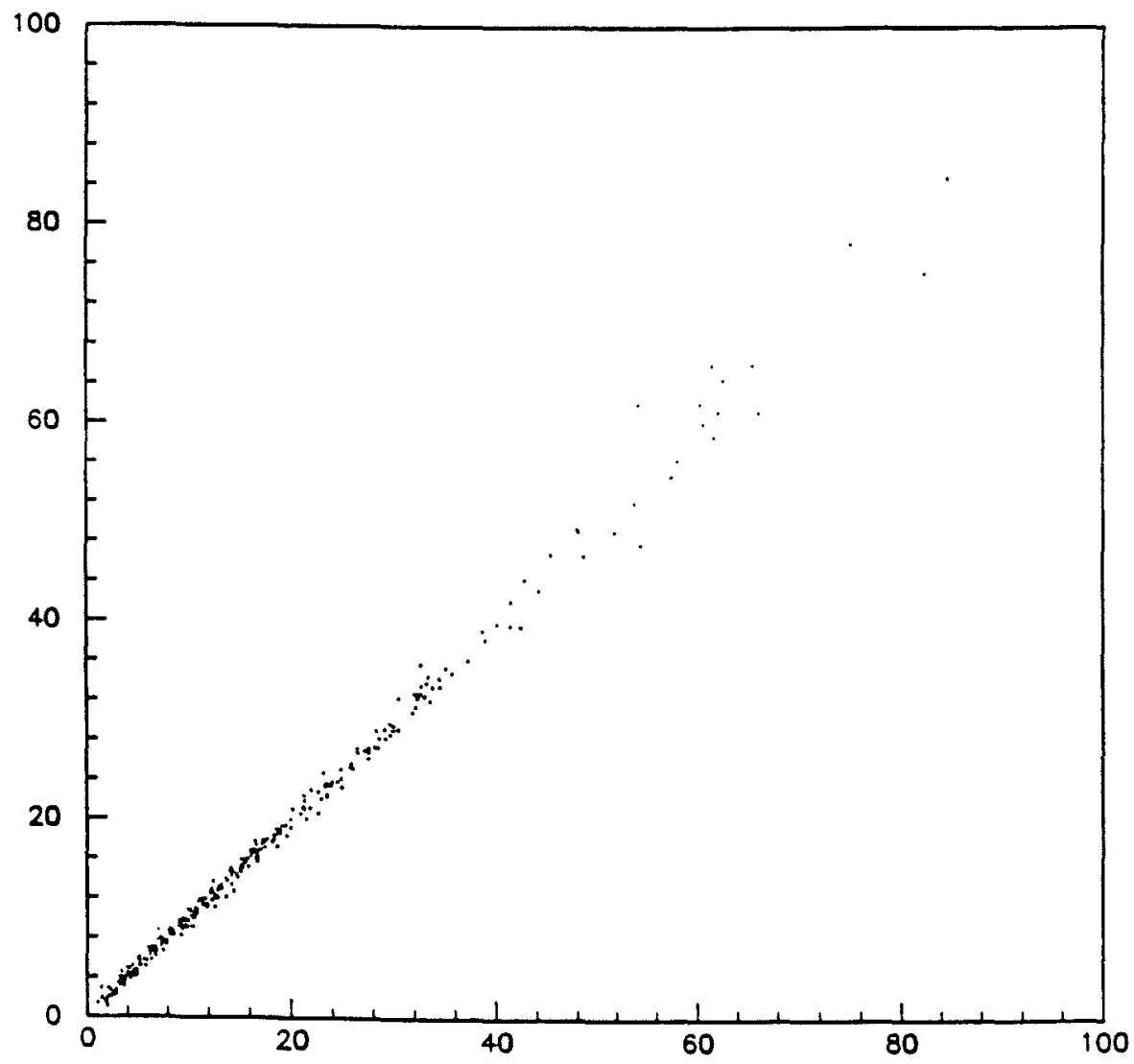

Figure 5.22: Generated versus Reconstructed momenta 

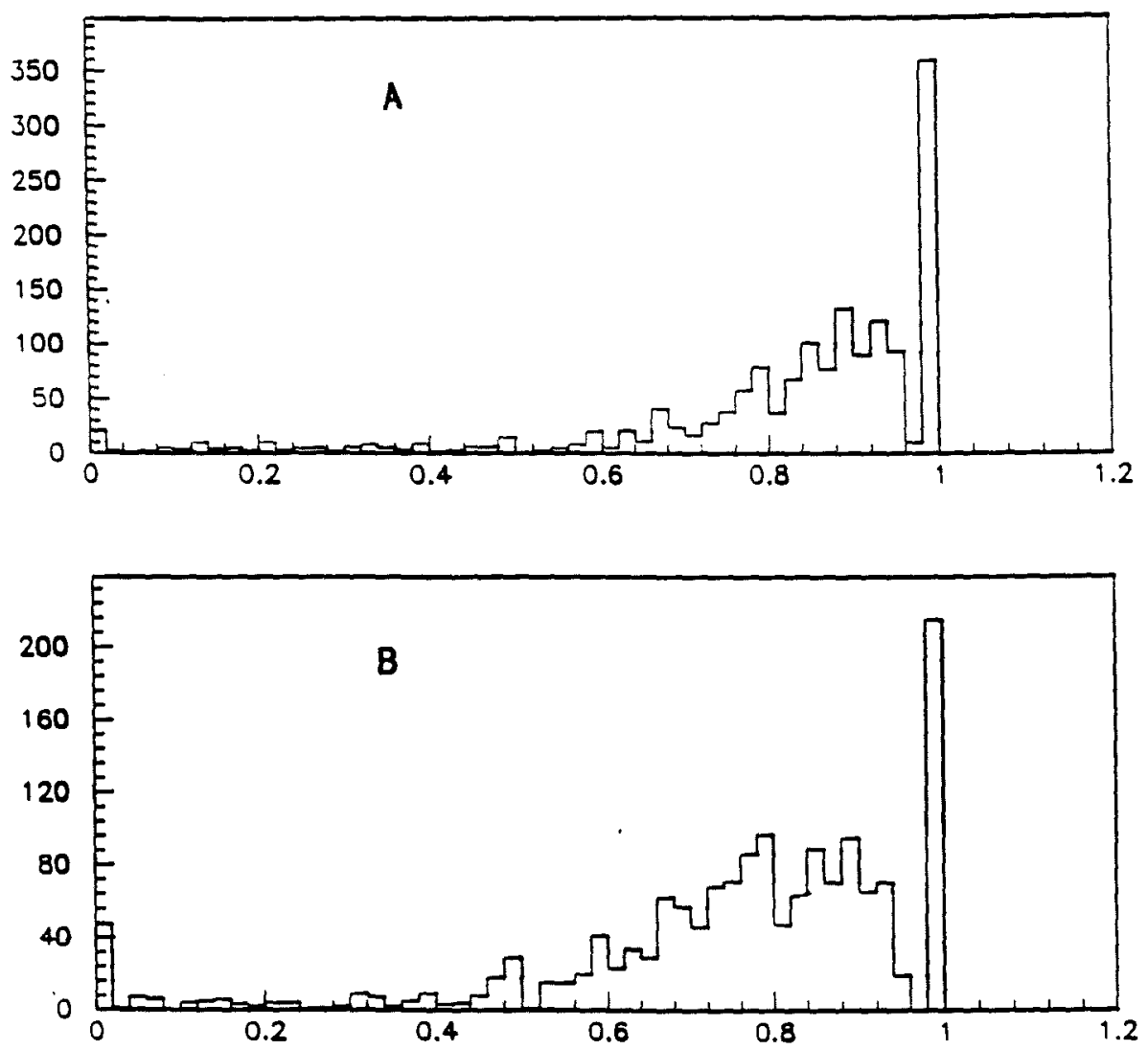

Figure 5.23: Fraction of the number of tracks linked in the events (A) in $\mathrm{X}$ view $(\mathrm{B})$ in $\mathrm{Y}$ view 


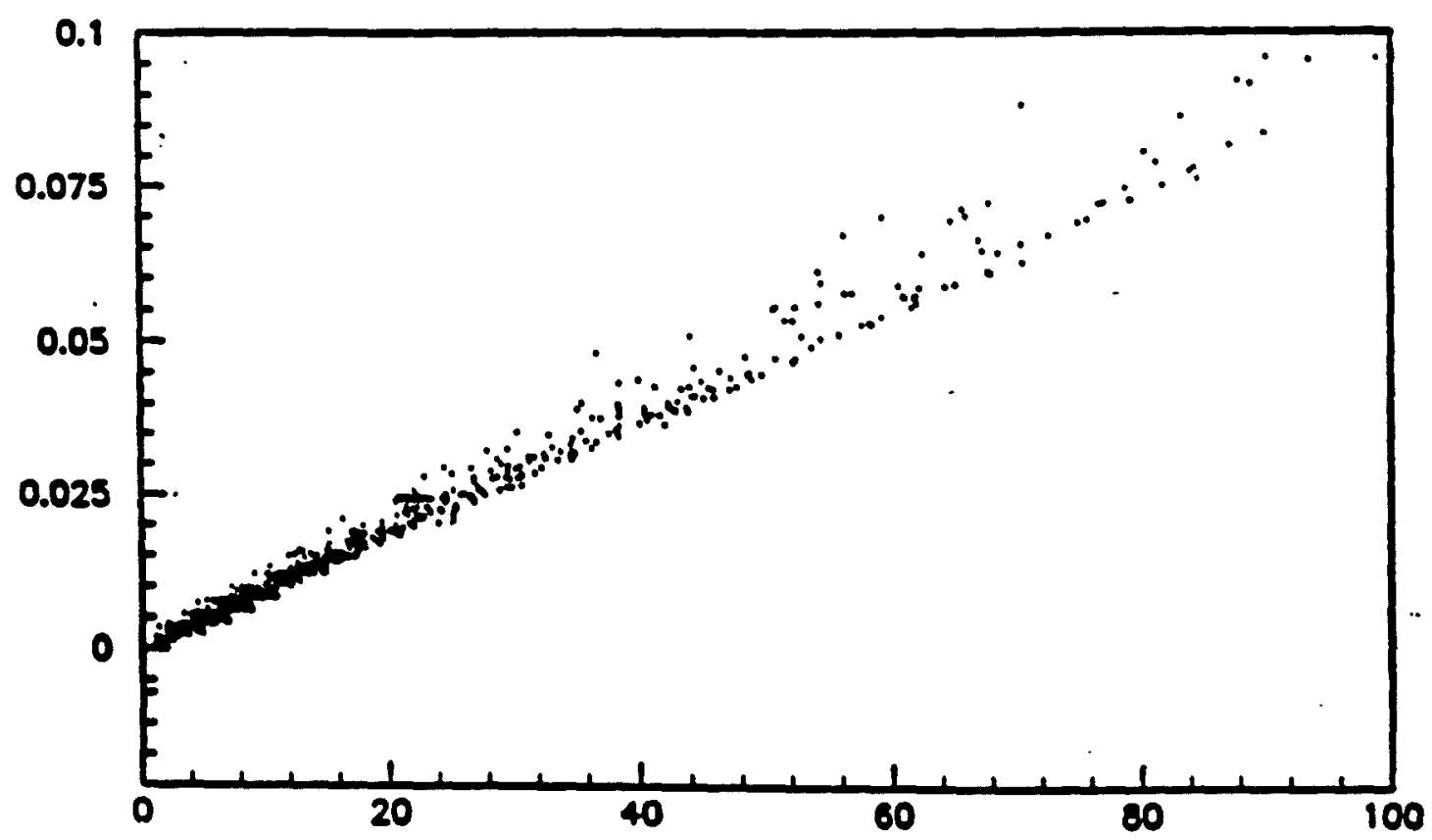

Figure 5.24: $\frac{\Delta P}{P}$ versus $\mathrm{P}$ for charged tracks 


\section{Chapter 6}

\section{Electromagnetic Shower Reconstruction}

A computer program reconstructs the electromagnetic shower positions and energies in the electromagnetic calorimeter [23]. For reconstruction purposes, the EMLAC is divided into four quadrants and each quadrant is divided into front and back sections. There is also a so-called sum section, which is the sum of the front and back sections. There are two views, namely, the r-view (azimuthal strips) and $\phi$-view (radial strips). The first $45^{\circ}$ sector of an r-view is called the left $\mathrm{r}$-view and the last $45^{\circ}$ sector of an $\mathrm{r}$-view is called the right $\mathrm{r}$-view. The $\phi$ view is divided into an inner $\phi$ region and an outer $\phi$ region. The reconstruction is done for each quadrant independently. This procedure consists of the steps described below.

\subsection{Group and peak finding in a given view}

The input to this program is an array of energy pulse heights in the channels (strips of the calorimeter). A group is a set of consecutive channels in a given view that have pulse heights above a certain threshold. The following 
conditions are necessary for a set of strips if they are to be designated as a group.

1. In the $\mathbf{r}$ and inner $\phi$ views, there must be at least 3 strips above an energy threshold. In the outer $\phi$ view this requirement is relaxed to having two or more strips above an energy threshold, since the strips are wider in this view.

2. The total group energy must be above $750 \mathrm{MeV}$.

3. The average energy per strip must be above $150 \mathrm{MeV}$.

4. The energy of the strip with the maximum energy must be at least $300 \mathrm{MeV}$.

According to the shape of the energy pulse height distribution in a group, one can have peaks and valleys of the pulse heights. A candidate for a peak is identified when the slope of the energy distribution in the group changes from a positive to a negative value. A valley is the low energy point between two peaks. It can also be the end strip in a group when the peak is the first or the last or the only peak in the group. Since in the sum section, the addition of the energy in the front and back sections can tend to obsure the existence of low energy peaks in the front of the calorimeter, a search for peaks between valleys is performed in the front section alone. If such a candidate for a peak is found, then a quantity $\sigma$ is calculated according to the following equation:

$$
\sigma=0.14^{2} E+0.01
$$


where $\mathrm{E}$ is the peak strip energy. This equation is based on Monte Carlo studies.

If the energy differences between the peak strip and its valleys on either side are greater than $2.5 \sigma$, then this candidate peak is considered as a real peak as opposed to an energy fluctuation.

\subsection{Shoulder finding in a peak}

At high $p_{T}$ and high energy a large fraction of the $\pi^{0}$ decay photons start to coalesce in every view. For instance, at $150 \mathrm{GeV}$ the minimum distance between two photons from a symmetric $\pi^{0}$ decay is only about $1.6 \mathrm{~cm}$. Also for a highly asymmetric $\pi^{0}$ decay, the low energy photon would appear as a shoulder to a peak in a group. The shoulder-finding algorithm tries to reconstruct the energy distribution in the above cases, as separate peaks. It looks for peaks and shoulders in the logarithmic energy derivatives of the adjacent strips. No shoulder is allowed next to a peak if it hits between two adjacent strips. A shoulder is only searched for in a single peak group. The code is optimized to ensure that no artificial shoulders are generated.

\section{3 $\gamma$ reconstruction}

In this chapter a $\gamma$ is defined as an energy distribution in a given view of a candidate shower. If a group consists of only one peak, it is considered a $\gamma$; but if it has several peaks, it has to be split into several 1-peak groups which can then be called $\gamma$ 's. 
This splitting procedure uses the well understood electromagnetic shower shape from Monte Carlo studies. The shower shapes in the front and back sections are parameterised by:

$$
\begin{gathered}
E_{f}(r)=\frac{1}{r}\left(f_{1} e^{-f_{2} r}+f_{3} e^{-f_{4} r}+f_{5} e^{-f_{6} r}\right) \\
E_{b}(r)=b_{1} e^{-b_{2} r}+b_{3} e^{-b_{4} r}+b_{5} e^{-b_{6} r}
\end{gathered}
$$

Here $r$ is the radius from the shower center. The other parameters in the above equations are determined from Monte Carlo studies. The total energy of a shower in the sum-section is:

$$
E_{\text {sum }}(r)=E_{f}(r)+E_{b}(r)
$$

The $\gamma$ position is calculated in two steps. First, the center position of each strip is calculated using the strip width and the location of each strip. Second, the offset of the $\gamma$ position from the center of the peak strip is calculated using a weighting scheme which uses the energy pulse height values on either side of the peak strip. In the radial view this offset is given by:

$$
X=1.702 \frac{s_{\text {left }}-s_{\text {right }}}{s_{\text {left }}+s_{\text {right }}}
$$

where $s_{\text {left }}$ and $s_{\text {right }}$ are the energies of the strips on either side of the peak strip. In the $\phi$ views a factor dependent on the estimated radius is used instead of 1.702 in the above equation. After this, several fits to the shower shape are done using values near this value of $X$ and the value of $X$ which gives the best fit is used to calulate the $\gamma$ location. 
The energy of the $\gamma$ is found by minimizing the $\chi^{2}$ of the fit of the shower shape to the actual pulse height distribution. The fitting is done by varying the energy of the shower.

If there are multiple peaks in a group, the peaks are first split and the energies and positions of the split peaks are then calculated. The splitting of the peaks is done by the following $\chi^{2}$ minimization method.

Let:

$e_{i}=$ energy in the $i^{t h}$ strip,

$f_{i k}=$ fraction of the energy in the $i^{\text {th }}$ strip due to the $k^{\text {th }}$ shower

$E_{k}=$ true energy of the $k^{t h}$ shower. The $\chi^{2}$ here is defined as:

$$
\chi^{2}=\sum_{i}\left(e_{i}-\sum_{k}\left(E_{k} f_{i k}\right)\right)^{2}
$$

Minimizing with respect to $E_{l}$ :

$$
\frac{d \chi^{2}}{d E_{l}}=0
$$

means:

$$
\sum_{i}\left(e_{i}-\sum_{k}\left(E_{k} f_{i k}\right)\right) f_{i l}=0
$$

to obtain:

$$
\sum_{k} E_{k} \sum_{i}\left(f_{i k} f_{i l}\right)=\sum_{i} e_{i} f_{i l}
$$

Let:

$$
S_{k l}=\sum_{i} f_{i k} f_{i l}
$$

and

$$
I_{l}=\sum_{k} E_{k} S_{k l}=\sum_{i} e_{i} f_{i l}
$$


then:

$$
E_{k}=I_{l}^{r} S_{k l}^{-1}
$$

This is an expression for the energy of the shower.

If the matrix $\mathrm{S}$ is not invertible, the peak of the lowest energy is dropped from the group and another attempt at splitting is made.

Once the energies of the individual $\gamma$ 's are known, they are used to calculate the corrected pulse distributions and a check on whether the peak strips are shifted is made. If they are shifted, the positions of the peaks are recalculated and the above procedure is repeated. The final $\chi^{2}$ used here is:

$$
\chi^{2}=\sum_{i}\left(\frac{e_{i}-\sum_{k} E_{k} f_{i k}}{\sigma_{i}}\right)^{2}
$$

where $\sigma_{i}$ is calculated based on the total contribution from all $\gamma^{\prime}$ 's to that strip.

\subsection{Correlation of $\gamma^{\prime} \mathrm{s}$ in $\mathbf{r}$ and $\phi$ view}

Showers reconstructed in both $\mathrm{r}$ and $\phi$ views are correlated by testing how close their energies and their ratios of the energies in the front and back sections are. The first step in this procedure is to identify which $\gamma$ 's lie near the octant and quadrant boundaries. This is done by comparing the first and last strips of all groups to see if any lie on the boundary. The correlation of these $\gamma$ 's is done first.

For the $\gamma$ 's away from the boundaries, the types of correlations attempted are one $\mathrm{R}$ to one $\phi$, one $\mathrm{R}$ to two $\phi$ and one $\phi$ to two $\mathrm{R}$. The 
one to one correlation is a simple matching of one $\mathrm{R} \gamma$ to an inner or outer $\phi \gamma$. In the one $\mathrm{R}$ to two $\phi$ correlation, each $\mathrm{R} \gamma$ is matched with a pair of inner $\phi \gamma$ 's or a pair of outer $\phi \gamma$ 's. Once a correlation is found, an attempt to split the $\mathrm{R} \gamma$ is made in order to check if there were actually two real showers in this situation. The one $\phi$ to two $R$ correlation is done essentially in the same fashion, but with the views reversed.

The correlated $\gamma$ 's are considered as real showers and their energies and locations are stored as the results of the electromagnetic shower reconstruction. The amount of energy in the $\gamma$ 's in the two views which are not correlated is summed up for each quadrant. This quantity is called the "uncorrelated energy." This quantity is also stored for later analysis. 


\section{Chapter 7}

\section{Data Reduction and Event Classification}

This chapter describes how the data used in the subsequent analysis are selected and how the events are classified according to the nature of the triggering particles. The term $p_{T}$ refers to the transverse momentum of a particle with respect to the beam direction.

\subsection{Event selection}

Those events which satisfied the following criteria were the only ones which were accepted for analysis:

1. There was a good beam particle definition from the beam counters and the event had to have a reconstructed beam track.

2. The event had to have a reconstructed vertex in the target region.

3. There was at least one charged track in the MWPC's and at least one reconstructed shower in the electromagnetic calorimeter. 
4. Since the jet structure is more pronounced at higher $p_{T}$, at first the events are selected such that they contain at least one photon shower or a diphoton shower (sum of two photon showers) with a $p_{T}$ greater than $4 \mathrm{GeV} / \mathrm{c}$.

5. In order to avoid events triggered by halo muons, events in which neither of the veto walls registers a hit in the quadrant with the highest $p_{T}$ photon, are selected.

6. The highest $p_{T}$ photon reconstructed in the event should be in the triggering octant.

7. There are muons which hit the calorimeter, but are not synchronised with the photon signal in the events. These muons are called out-oftime muons. The time of arrival of the showers are recorded during data acquisition. The highest $p_{T}$ photon is required to be hitting the calorimeter at nearly the same time as the time as the interaction occurred.

8. The energy of the highest energy photon is required to be less than $150 \mathrm{GeV}$, so that it could not be confused with a coalescence of a $\pi^{0}$ decay.

9. The maximum uncorrelated energy in any quadrant is required to be less than $10 \mathrm{GeV}$.

10. The maximum number of showers in an event is set at 40 . The selection of individual showers is described later. 
11. The highest $p_{T}$ shower is required to be within the geometrical fiducial region of the calorimeter and not near its boundaries.

12. The maximum number of charged tracks in an event is set at 40 . The selection of the individual tracks is described later.

\subsection{Shower selection}

Only those showers in the electromagnetic calorimeter which satisfied the following requirements were considered for analysis. These requirements were geared towards taking photons coming from the vertex region for the analysis.

1. They had to be within the fiducial region of the calorimeter.

2. The ratio of the energy in the front section of the calorimeter to that in the back section had to be greater than 1.5. This ratio cut is intended to discard hadronic showers being considered as photon showers.

3. The shower should not match within $1 \mathrm{~cm}$ with a track at the face of the calorimeter. This is to avoid double counting of hadrons or electrons in the tracking system and the calorimeter.

\subsection{Charged track selection}

Only those tracks which satisfied the following criteria were considered for analysis. 
1. Their momentum had to be lower than $150 \mathrm{GeV} / \mathrm{c}$. Above this value, the uncertainty in the momentum determination was too large.

2. When projected to the target region the track had to be within $1 \mathrm{~cm}$ of the reconstructed vertex. This was employed so as to select only those tracks which were created as a result of the primary interaction.

3. If the track matched (within $1 \mathrm{~cm}$ ) with a shower in the calorimeter and if the ratio of its energy in the calorimeter to its momentum was between 0.8 and 1.2 and if the ratio of the energy in the front of the calorimeter to that in the back of the calorimeter was greater than 0.4 , then it was quite likely to be an electron. Electrons were removed from this analysis.

\section{$7.4 \quad \pi^{0}$ event selection}

Since the main signals observed by the electromagnetic calorimeter were $\pi^{0}$ 's and direct $\gamma$ 's, the events were to be classified as $\pi^{0}$ events and direct photon events.

The $\pi^{0}$ reconstruction was done to find the highest $p_{T} \pi^{0}$ in the event and to see whether the highest $p_{T}$ shower in the calorimeter which formed the trigger of the experiment was actually from the decay of a $\pi^{0}$. In order to reconstruct $\pi^{0}$ s, all of the pairs of showers were considered from the electromagnetic calorimeter. If the invariant mass of these pairs was 
between 100 and $170 \mathrm{MeV}$, it was considered as a $\pi^{0}$ and if it was between 500 and $600 \mathrm{MeV}$ then it was considered as an $\eta$.

In order for the event to be considered a good $\pi^{0}$ event, it had to satisfy the following requirements.

1. Both of the showers had to pass the shower selection criteria.

2. The highest $p_{T}$ neutral object in the event had to be a $\pi^{0}$.

3. The $\pi^{0}$ had to have a $p_{T}$ of at least $4 \mathrm{GeV} / \mathrm{c}$.

4. The highest $p_{T} \pi^{0}$ had to be reconstructed from the highest $p_{T}$ photon in the event.

5. Let $E_{1}$ and $E_{2}$ be the energies of the two photons which satisfied the $\pi^{0}$ mass cut. The asymmetry of the $\pi^{0}$ decay is defined as:

$$
A=\frac{\left|E_{1}-E_{2}\right|}{E_{1}+E_{2}}
$$

This is approximately the cosine of the angle either of the photons makes in the rest frame of the $\pi^{0}$. Figure 7.1 shows the asymmetry of the pizeros. For the $\pi^{0}$ s which decay symmetrically this quantity would be zero. For those $\pi^{0}$ 's which have a high asymmetry value, it is possible that one of the photons could be outside the acceptance of the calorimeter. Hence the photon which is detected could accidently make a $\pi^{0}$ mass combination with another photon. Hence the $\pi^{0}$ event selected is required to have a $\pi^{0}$ asymmetry less than 0.75 . 


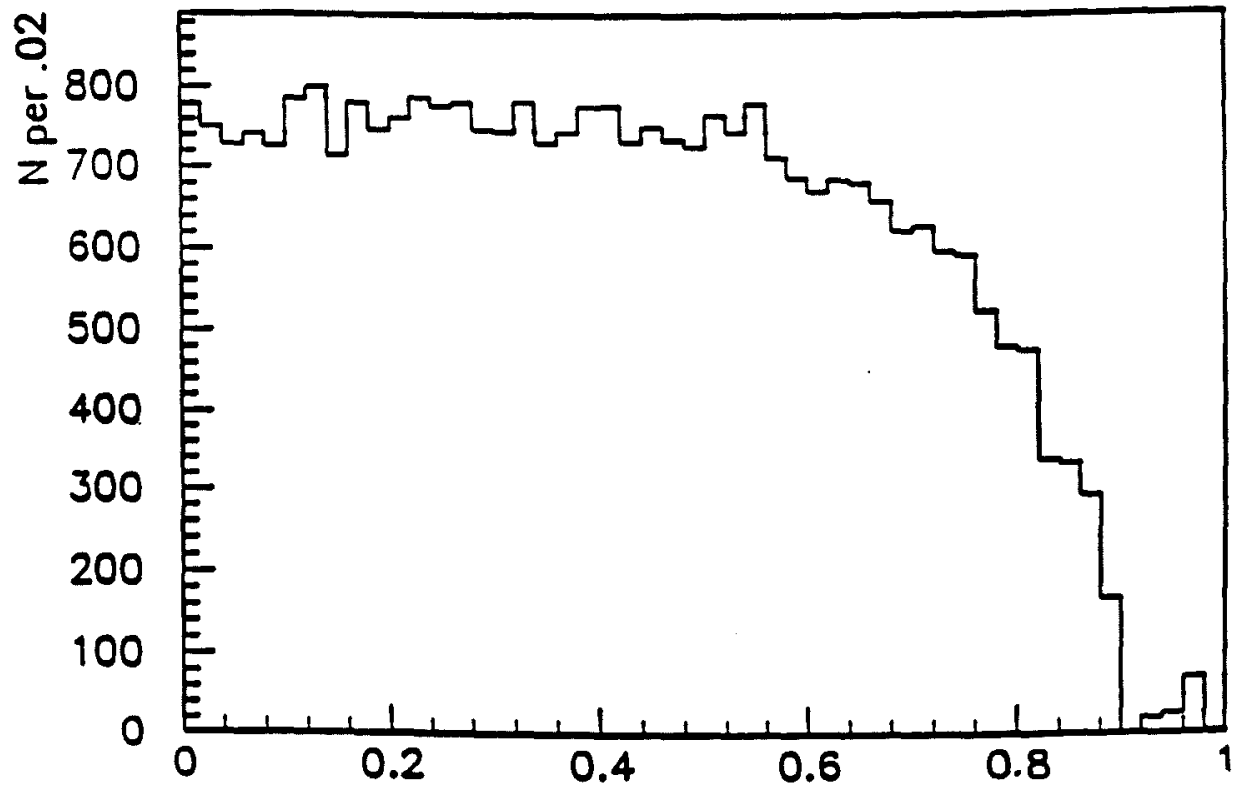

Figure 7.1: Asymmetry of pizeros 
6. Another useful variable named directionality is defined as:

$$
d=F_{r}-B_{r} \frac{F_{z}-T_{z}}{B_{z}-V_{z}}
$$

where:

$F r=$ radial position of a shower at the front face of the LAC

$B_{r}=$ radial position of a shower at the back face of the LAC

$F_{z}=$ z coordinate of a shower at the front face of the LAC

$B_{z}=\mathrm{z}$ coordinate of a shower at the back face of the LAC

$V_{z}=\mathrm{z}$ coordinate of the matched vertex in the event

For particles which come from the vertex the directionality is zero. In order to ensure that a shower in the event came from the vertex and not from a halo muon, each shower is required to have a directionality less than 0.4 . For showers in which there was no energy detected in the back section of the calorimeter, the directionality could not be calculated and hence this cut could not be applied. Figure 7.2 shows the schematic diagram of a shower in the calorimeter and illustrates how the directionality is calculated. For the showers which are due to out-of-time muons the directionality values are non-zero as seen in Figure 7.3.

7. Only those events in which the highest $p_{T}$ pizero was within a rapidity range of -0.7 to 0.7 was considered for jet reconstruction. Outside these limits the acceptance of the highest $p_{T}$ shower was very small. 


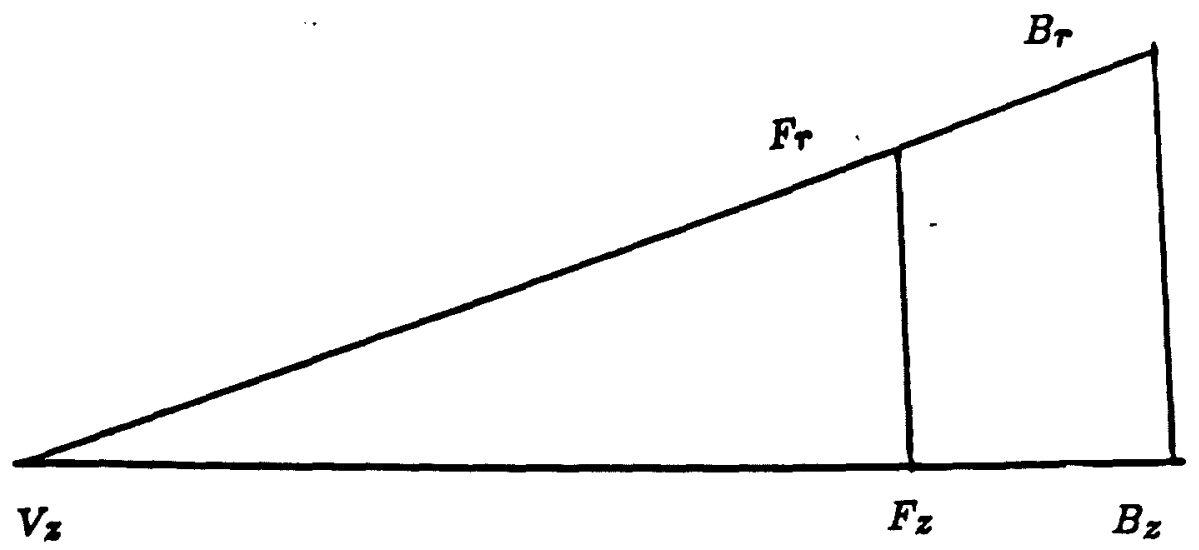

Figure 7.2: Illustration of directionality 


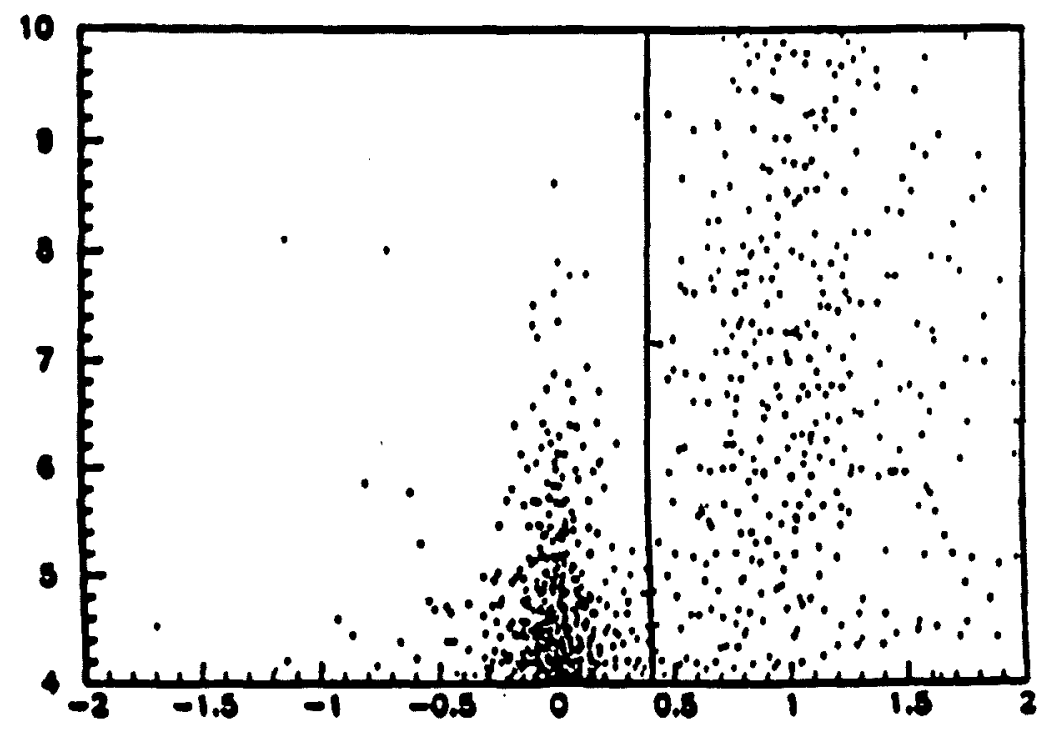

Figure 7.3: $p_{T}$ versus directionality of showers 


\subsection{Direct photon candidate selection}

For the direct photon candidate events in addition to the shower selection criteria, the following selection criteria were applied.

1. The highest $p_{T}$ photon should not make a $\pi^{0}$ or $\eta$ or any mass less than $175 \mathrm{MeV}$ when combined with any other photon for the invariant mass calculation.

2. The highest $p_{T}$ photon should not be one which was reconstructed from a $2 \mathrm{R}$ to $1 \phi$ or $2 \phi$ to $1 \mathrm{R}$ correlation in the electromagnetic shower reconstructor.

3. The highest $p_{T}$ photon should have a directionality less than 0.4 .

4. Only those events in which the highest $p_{T}$ photon was within a rapidity range of -0.7 to 0.7 was considered for jet reconstruction. Outside these limits the acceptance of the highest $p_{T}$ shower was very small.

The highest $p_{T}$ shower in the direct photon candidate event is considered as the direct photon.

\subsection{Event Analysis}

In the following chapter the data will be analysed in terms of a jet analysis. Comparisons will be made between direct photon events and $\pi^{0}$ events and between E706 data and data from Monte Carlo events. The events to be 
Table 7.1: Numbers of events of various types used in the analysis

\begin{tabular}{|c|c|c|c|c|}
\hline $\begin{array}{l}\text { Target and } \\
\text { Beam Type }\end{array}$ & $\begin{array}{c}\gamma \\
\text { events }\end{array}$ & $\begin{array}{c}\pi^{0} \\
\text { events }\end{array}$ & $\begin{array}{l}\gamma \text {-jet } \\
\text { events }\end{array}$ & $\begin{array}{l}\pi^{0} \text {-jet } \\
\text { events }\end{array}$ \\
\hline $\mathrm{Cu}-\mathrm{Be} \mathrm{Neg}$ & 4740 & 9570 & 2790 & 5780 \\
\hline $\mathrm{Cu}-\mathrm{Be} \mathrm{Pos}$ & 5780 & 9380 & 3590 & 6030 \\
\hline $\mathrm{Be} \mathrm{Neg}$ & 4580 & 7270 & 2700 & 4640 \\
\hline
\end{tabular}

used are the ones which satisfy the criteria discussed in the present chapter.

The number of $\gamma$ and $\pi^{0}$ events are shown in Table 7.1 . 


\section{Chapter 8}

\section{Analysis of Jet Events}

\subsection{Evidence of jet structure}

As discussed in the introduction particle production at high transverse momenta is expected to show hard scattering effects with the partons hadronizing as hadronic jets. In this chapter the evidence for jet structure will be presented. Following this a more quantitative analysis involving a jet finding algorithm is described. Finally the results of this analysis are presented in terms of the structure and fragmentation functions.

In Figure 8.1 the $p_{T}$ vectors of the particles in the $\mathrm{XY}$ plane (transverse to the beam direction) in a high $p_{T}$ direct photon candidate event are shown. The length of the vectors are proportional to the $p_{T}$ of the individual particles. The clustering of the vectors in this figure indicates the jet structure in the event. Figure 8.2 shows a lego plot of rapidity versus

azimuthal angle for each of the observed particles from a single high $p_{T} \pi^{0}$ event. The height of each tower is proportional to the $p_{T}$ of each particle in the event. It is clear that the $\pi^{0}$ is balanced by several particles (a jet) 
at the same rapidity but separated by $180^{\circ}$ in azimuthal angle. In both of these figures the strong back-to-back correlation of trigger jet and recoil jet is evident.

For a statistical analysis, in Figure $8.3[28]$ the azimuthal difference between the direction of the trigger particle and that of the rest of the particles in the event is shown. The sharp rise near $180^{\circ}$ is due to the fact that in the transverse plane the recoil jet is directed opposite to that of the trigger particle. Note that this occurs for both direct photon and $\pi^{0}$ events and suggests that the trigger particle $p_{T}$ is balanced by particles going $180^{\circ}$ to the trigger particle. The fact that the particles are collimated suggests a jet-like structure.

There are also some particles in the direction of the trigger particle when it is a photon which are probably due to the Bremsstrahlung photons. In the case of the $\pi^{0}$ triggered events, there is a significant increase in the number of particles on the same side. The dotted lines in Figure 8.3, from minimum bias events, reveal the sharp contrast between the hard-scattering process and the "ordinary" (low $p_{T}$ ) hadronic interactions. The particle multiplicity is also significantly higher in the high $p_{T}$ events than in the minimum bias events.

In Figure 8.4(a) the rapidity difference between the leading and nextleading particles is shown. The leading particle is the particle with the highest $p_{T}$ after the trigger particle, and the next-leading particle is the particle with the highest $p_{T}$ after the leading particle. The increase in 


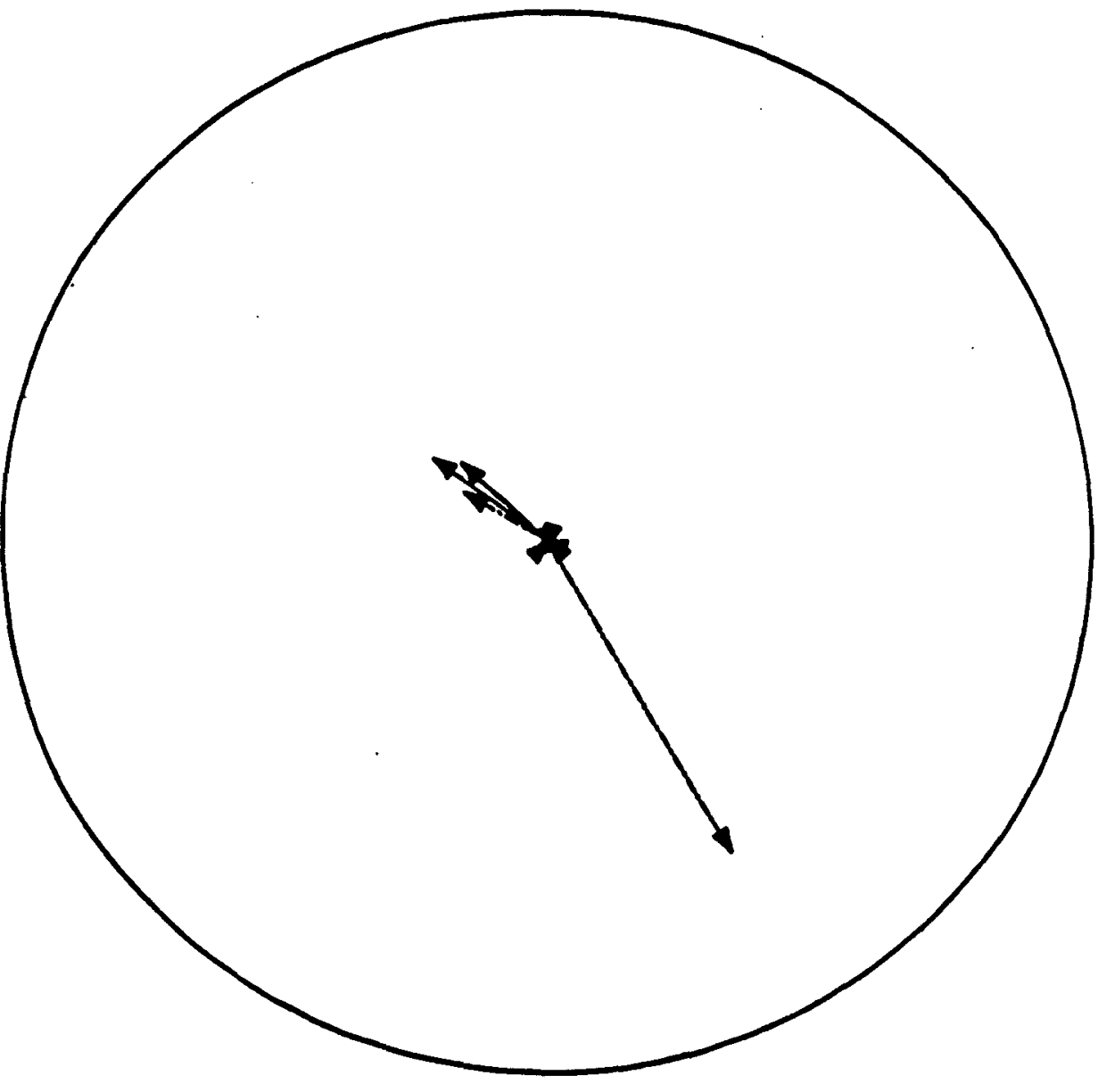

Figure 8.1: $p_{T}$ vectors

140 


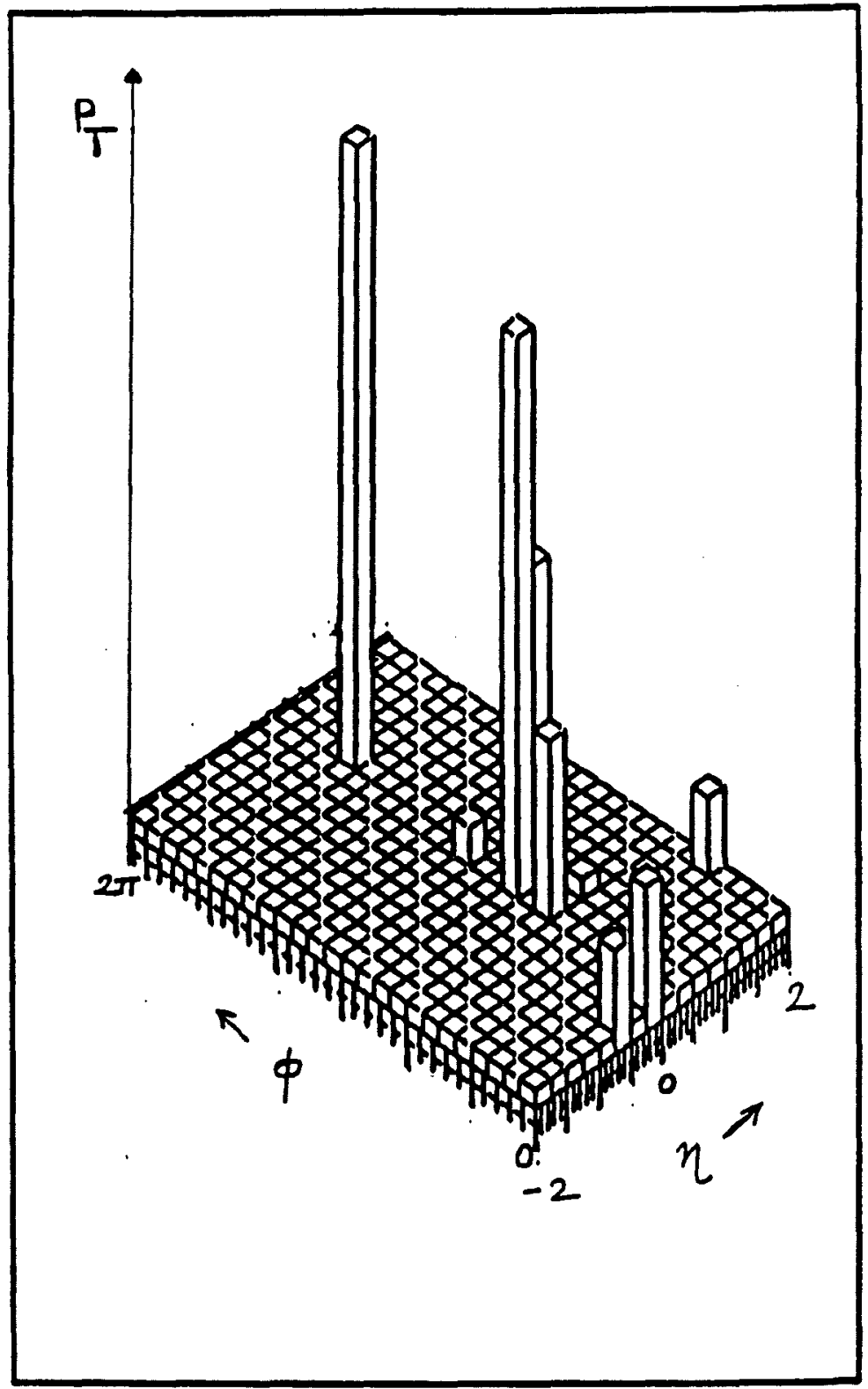

Figure 8.2: Rapidity versus azimuthal angle 


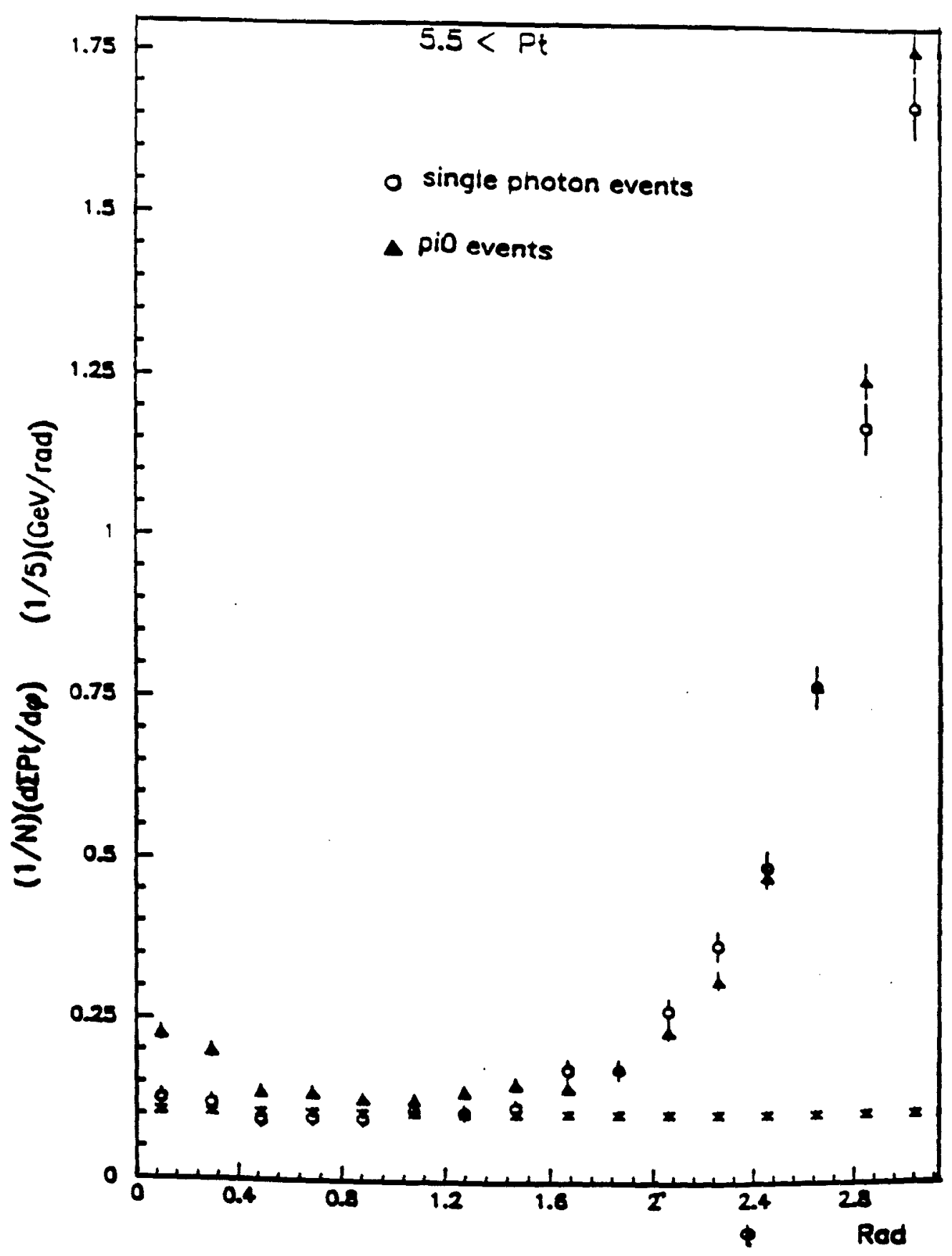

Figure 8.3: Azimuthal difference between particles in the event 
the rapidity difference around zero is an indication of the fact that the two highest $p_{T}$ particles are correlated in rapidity. Figure $8.4(\mathrm{~b})$ is the rapidity difference between that of the leading particle from one event and the next-leading particle from another event picked randomly. This forms a background level to Figure $8.4(\mathrm{a})$.

Given these preliminary suggestions of jet structure in these high $p_{T}$ events, a more quantitative analysis requires an algorithm to measure the amount of jet-like structure and to determine which particles belong to which jets. In the next section the algorithm for the jet reconstruction is discussed.

\subsection{Jet reconstruction}

For this analysis all charged tracks are assumed to be pions and all neutral particles are assumed to be photons. This algorithm, based on the discussion in reference[7], makes use of the incident beam direction and the fact that the fragments of the beam and target jets should have a limited momentum transverse to this direction whereas the trigger (or recoil) jet fragments should have limited transverse momentum with respect to the trigger (or recoil) jet axis.

For a given particle a weight is defined with respect to a chosen axis according to the formula:

$$
\boldsymbol{w}=\frac{p_{\|}}{p} e^{-5.0 p_{T}}
$$



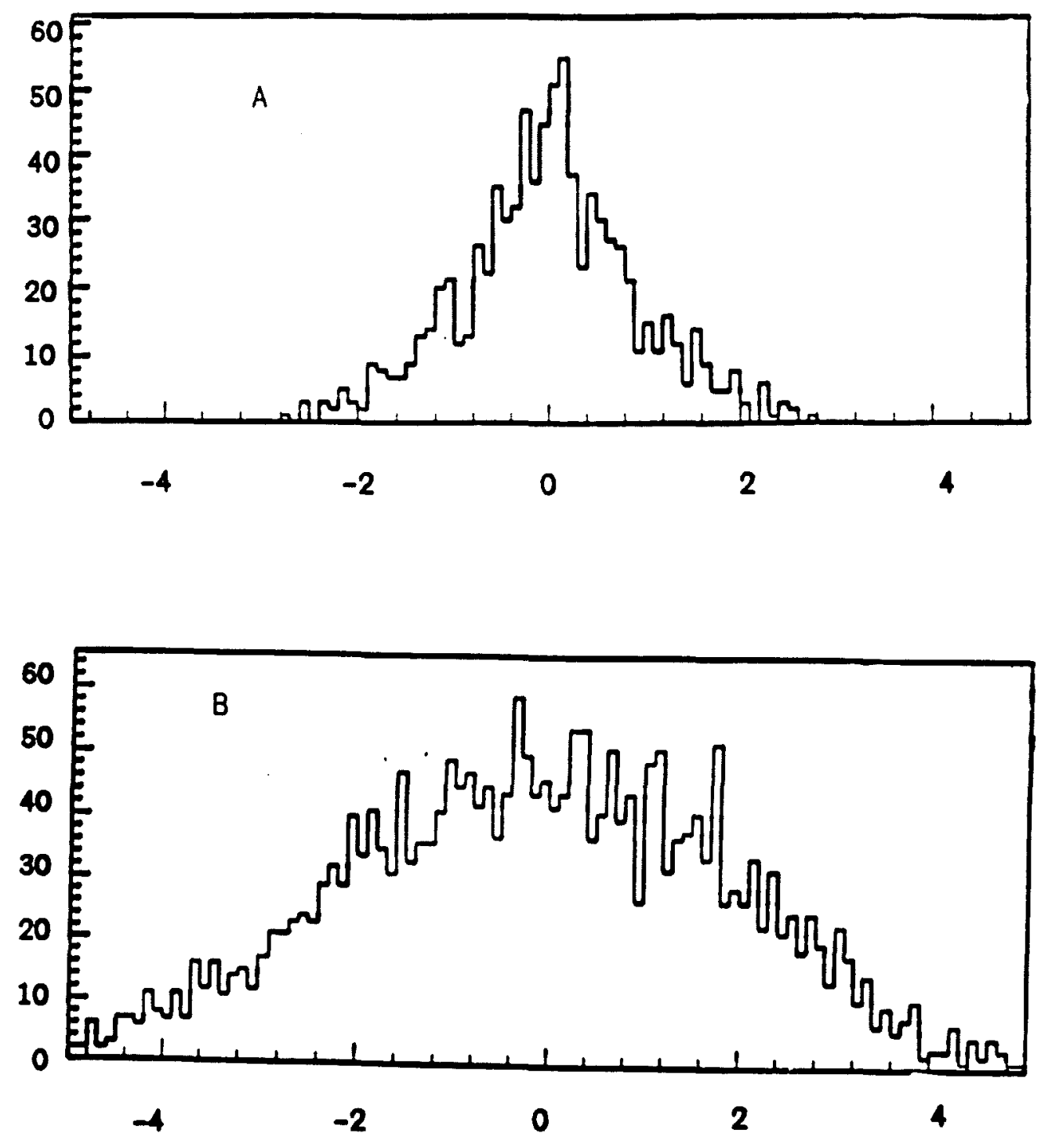

Figure 8.4: Rapidity difference between leading and next leading particles 
where:

$p_{\|}=$component of the momentum of the particle in the direction of an axis

$p_{T}=$ component of the momentum of the particle perpendicular to the direction of an axis

$p=$ total momentum of the particle

There are three axes in total, namely, the beam/target axis (axis 1 ), the recoil axis (axis 2), and the trigger axis (axis 3 ). Hence for every particle there are three weights $w_{1}, w_{2}, w_{3}$.

The initial seed for axis 1 is the beam direction, that for axis 2 is the direction of the leading particle in the event, and that for axis 3 is the direction of the trigger particle.

The probability of the particle being in a certain direction, say, along axis 1 is given by:

$$
P=\frac{w_{1}}{w_{1}+w_{2}+w_{3}}
$$

The jet direction is then determined by summing the vectors of all particles with $P>0.34$. A second iteration is then performed using the above determined jet directions as input.

Finally a jet event is selected using the following cuts.

- There must be at least two particles in the recoil jet.

- The transverse momentum of the jet must be greater than $20 \%$ of the transverse momentum of the trigger. 
- The $\phi$ angle between the trigger jet direction and the recoil jet direction must be at least $120^{\circ}$.

- The rapidities of the trigger jet and recoil jet must be between -1.0 and 1.0. This is to avoid those events where the trigger or recoil jet overlaps with the beam or target jet.

The momentum of a jet is found by summing the momenta of all particles assigned to that jet. The momentum of the recoil jet is then scaled such that the trigger and recoil jets have the same transverse momentum with respect to the beam direction. This ignores any motion of the partons normal to the direction of motion of the hadrons and any losses due to reconstruction. The events which are considered as jet events were used for further analysis. This procedure was done for direct photon triggered events and $\pi^{0}$ triggered events both in real data and Monte Carlo. There were two types of beam particles available, namely $\pi^{-}$and protons. The analysis was done separately on these. The numbers of events found by this jet algorithm are shown in Table 7.1. The results of this procedure are discussed in the following sections along with the results from other experiments.

The main purpose of the jet reconstruction that has been performed is to determine the direction of the jets and to identify which particles are in each jet. To check the efficiency of this algorithm an identical analysis has been performed on Monte Carlo data where one knows the direction of the generated jets. Figure 8.5 shows the rapidity and azimuthal angle of the reconstructed recoil jet versus the same variables for the generated recoil jet 
in the Monte Carlo data. These figures indicate that the generated azimuth and rapidity of the jet are reconstructed accurately. The efficiency of the jet reconstruction algorithm is defined as the ratio of the number of reconstructed jet events to the number of events selected for jet reconstrution. This efficiency is determined to be about $90 \%$ in the Monte Carlo data.

\subsection{Results of jet reconstruction}

In Figure 8.6(a) the $\phi$ of the leading particle versus that of the recoil jet is plotted. In Figure 8.6(b) the rapidity of the leading particle versus that of the reconstructed recoil jet is plotted. The fact that the $\phi$ and rapidity of the leading particle and those of the recoil jet are highly correlated is an indication that the reconstructed jet direction is dominated by the leading particle.

As indicated in Chapter 7 only those events where the rapidity of the trigger particle is between 0.7 and -0.7 were considered for jet reconstruction. Figure 8.7 shows the rapidity distributions of the trigger jets. Figure 8.7 shows the rapidity distributions of the recoil jets.

Figure 8.9 and Figure 8.10 show the recoil jet multiplicities for the positive and negative data for $\pi^{0}$ events and Figure 8.11 and Figure 8.12 show the same for $\gamma$ events. Note that there are typically 4 or 5 particles (charged pions or photons) in the recoil jet, with the distribution extending out to about 14. Similarly Figure 8.13 and Figure 8.14 show the trigger jet multiplicity for $\pi^{0}$ events and Figure 8.15 and Figure 8.16 show the same for $\gamma$ 

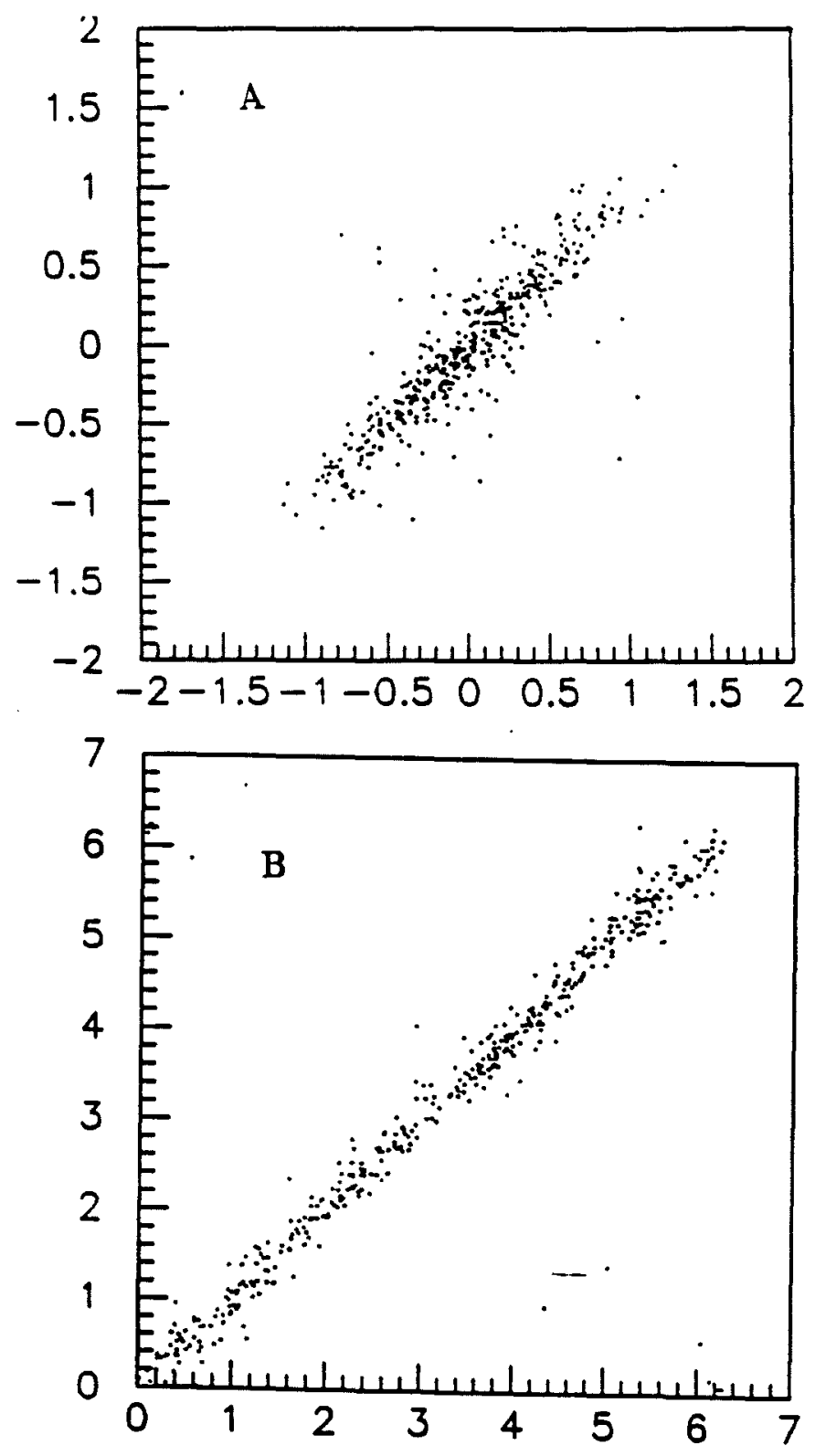

Figure 8.5: (A)Rapidity of generated versus reconstructed jets (B) Azimuthal angle of generated versus reconstructed jets 

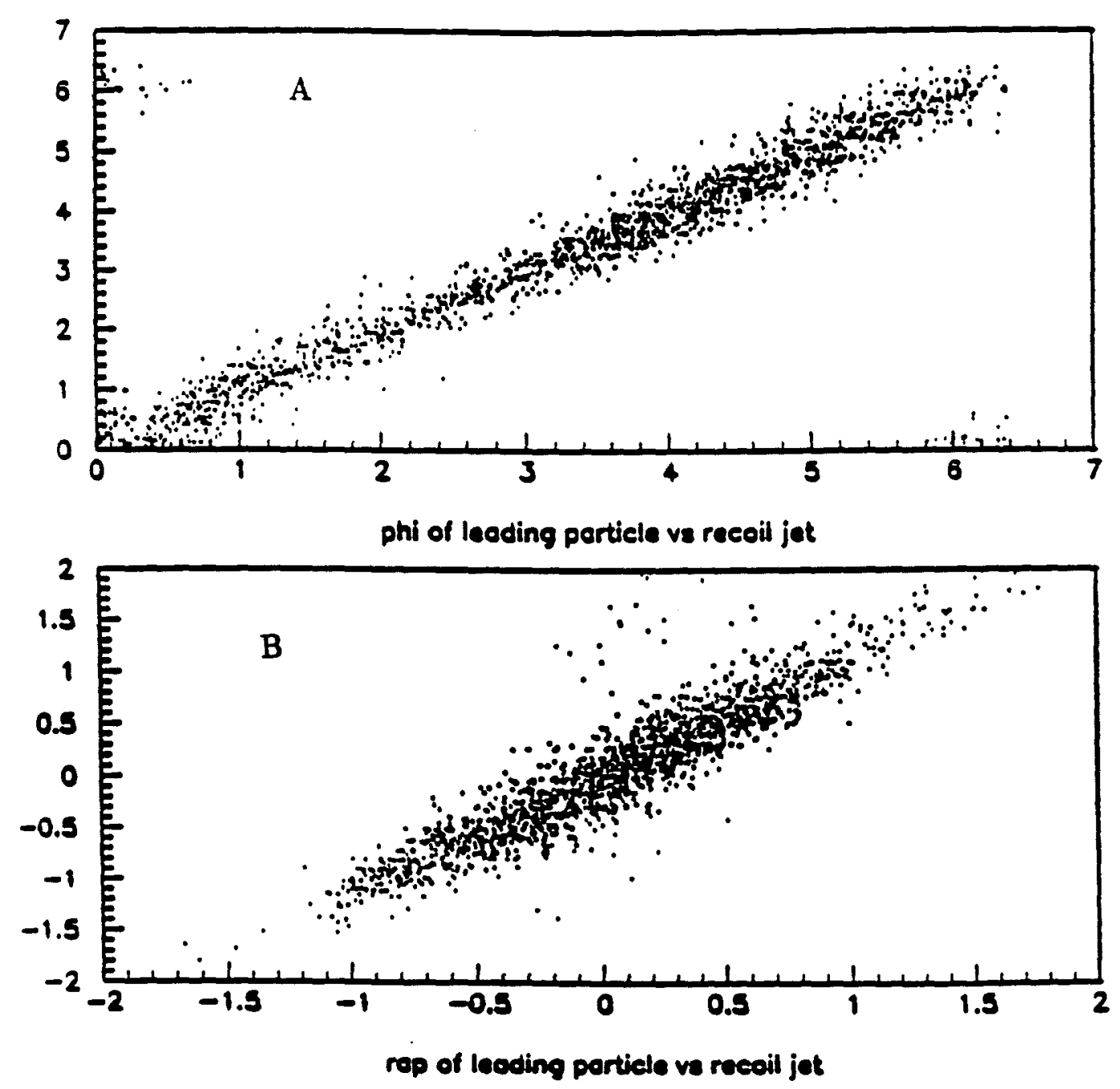

Figure 8.6: (A) $\phi$ of leading particle versus that of recoil jet (B) Rapidity of leading particle versus that of recoil jet 
events. The trigger jet multiplicities are smaller than those of the recoil jets. Figure 8.17 shows the angle of the recoil particles with respect to the recoil direction. This distribution peaks at about $20^{\circ}$ and this is a measure of the breadth of the recoil jets. Figure 8.18 shows the transverse momentum of the recoil particles with respect to the recoil direction. This distribution peaks at about $0.1 \mathrm{Gev}$ and this is another measure of the breadth of the recoil jets.

\subsection{Parton distributions}

In those events which have been determined to have a jet structure, (see Table 7.1) the longitudinal momentum fractions $x_{a}$ and $x_{b}$ mentioned in Chapter 1 are calculated. These quantities give a measure of the hardness of the parton level reaction. Monte Carlo data have been generated using the ISAJET and GEANT packages, passed through the reconstruction program and had the same selection criteria applied. Figure 8.19 and Figure 8.20 show these distributions for the photon triggered events in the E706 data. Figure 8.21 and Figure 8.22 show these distributions for the $\pi^{0}$ triggered events in the E706 data. The distributions from single photon events (direct

photon candidate events) and $\pi^{0}$ seem similar. Note that the data peak between 0.25 and 0.50 , indicating that for the $\sqrt{s}$ and $p_{T}$ available to E706 this is the region that E706 is most sensitive (compare Figure 8.19 and Figure 8.21 with Figure 1.8). Figure 8.23 shows these distributions for 


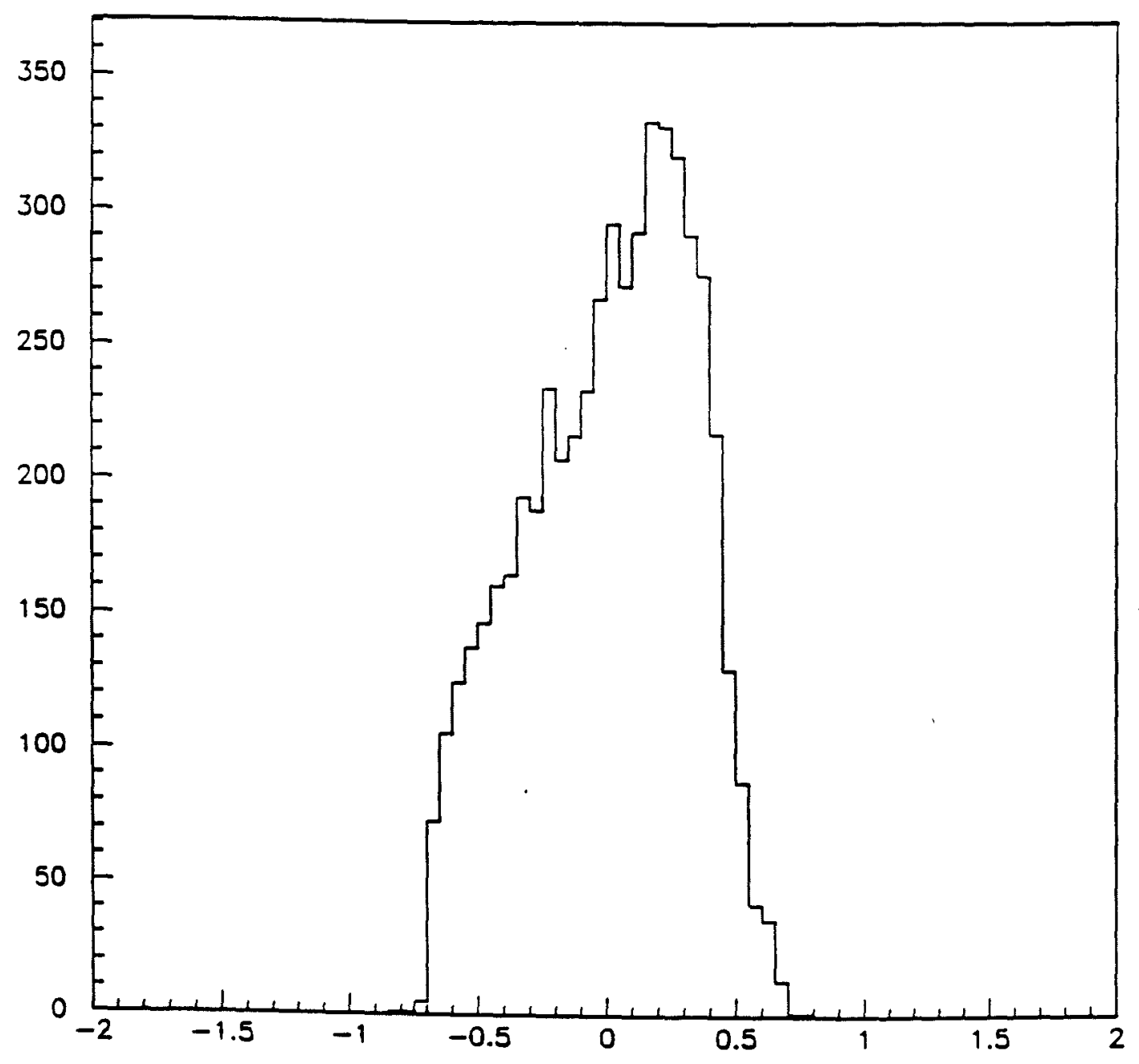

Figure 8.7: Rapidity distribution of trigger jet in single $\gamma$ events 


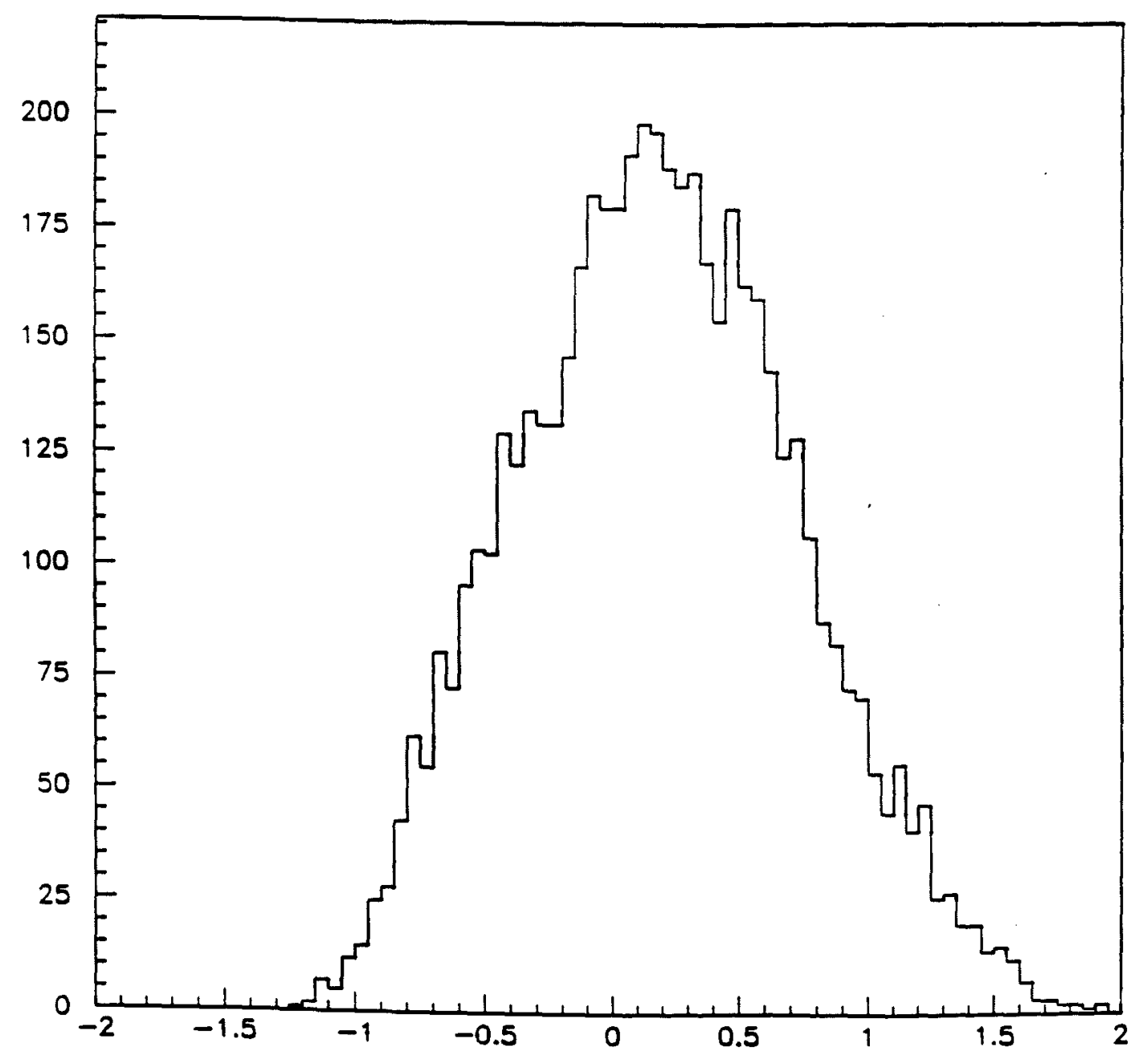

Figure 8.8: Rapidity distribution of recoil jet in single $\gamma$ events 


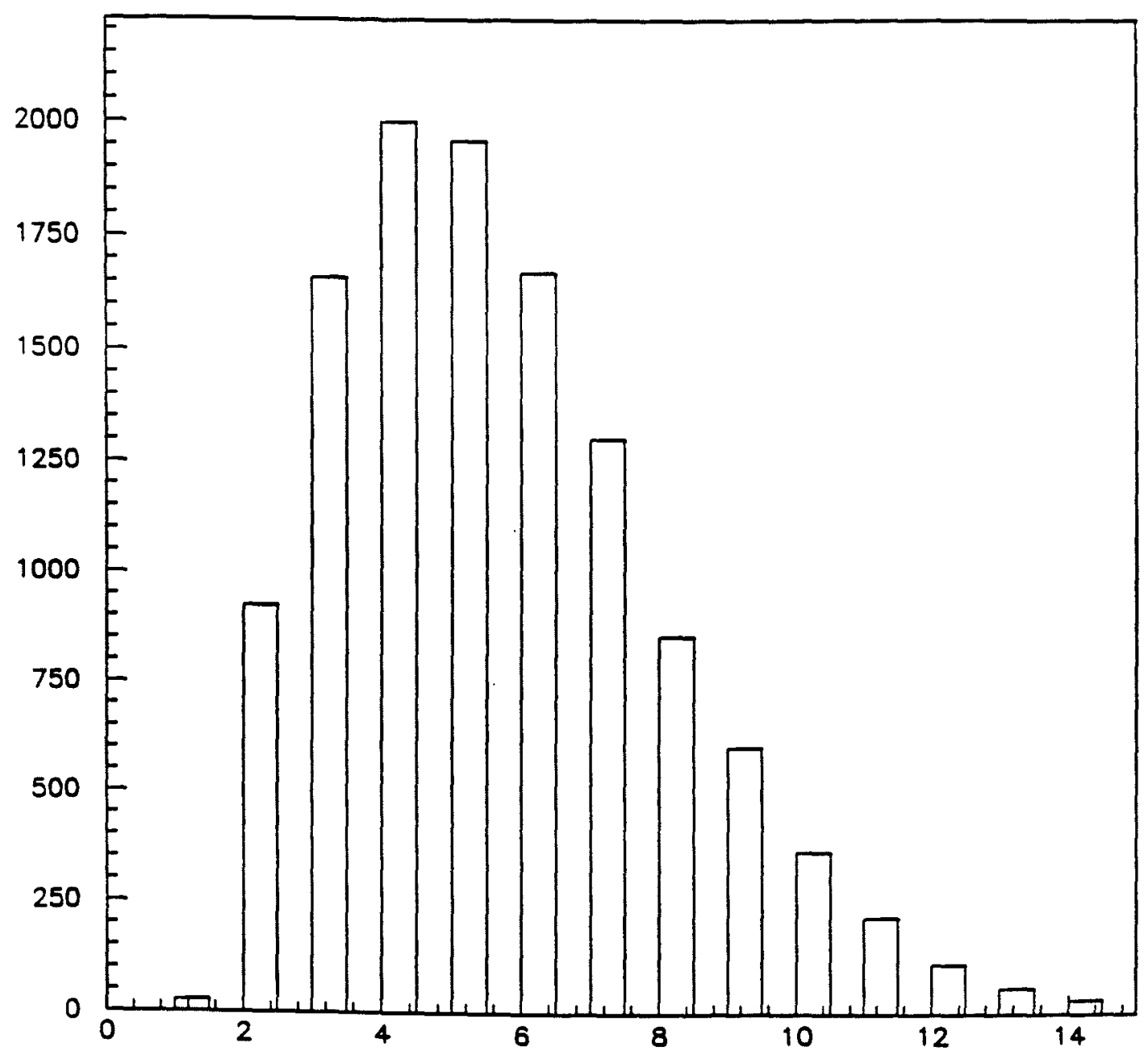

Figure 8.9: Recoil jet multiplicity in $\pi^{0}$ events in $\pi^{-}$data 


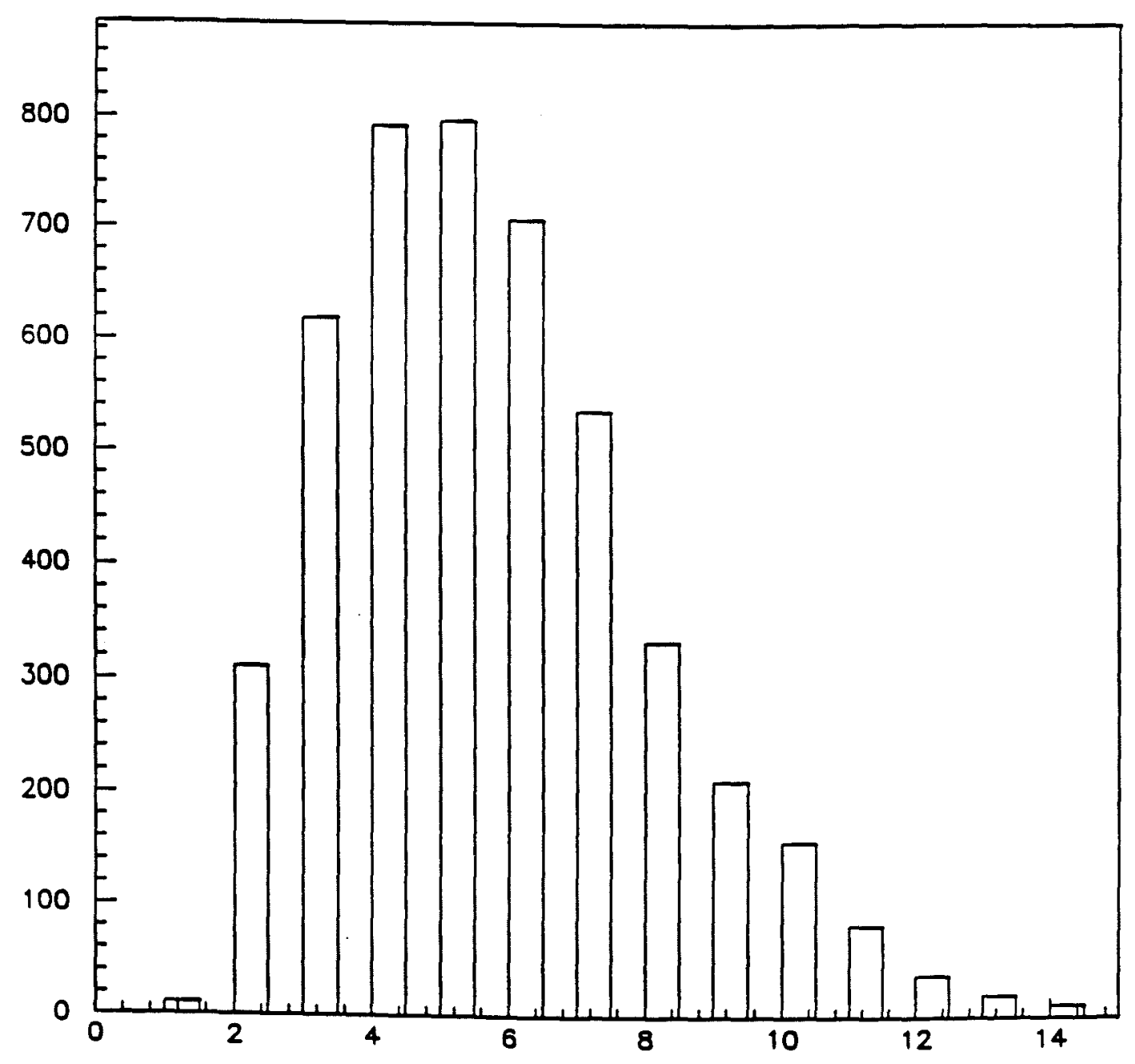

Figure 8.10: Recoil jet multiplicity in $\pi^{0}$ events in proton data 


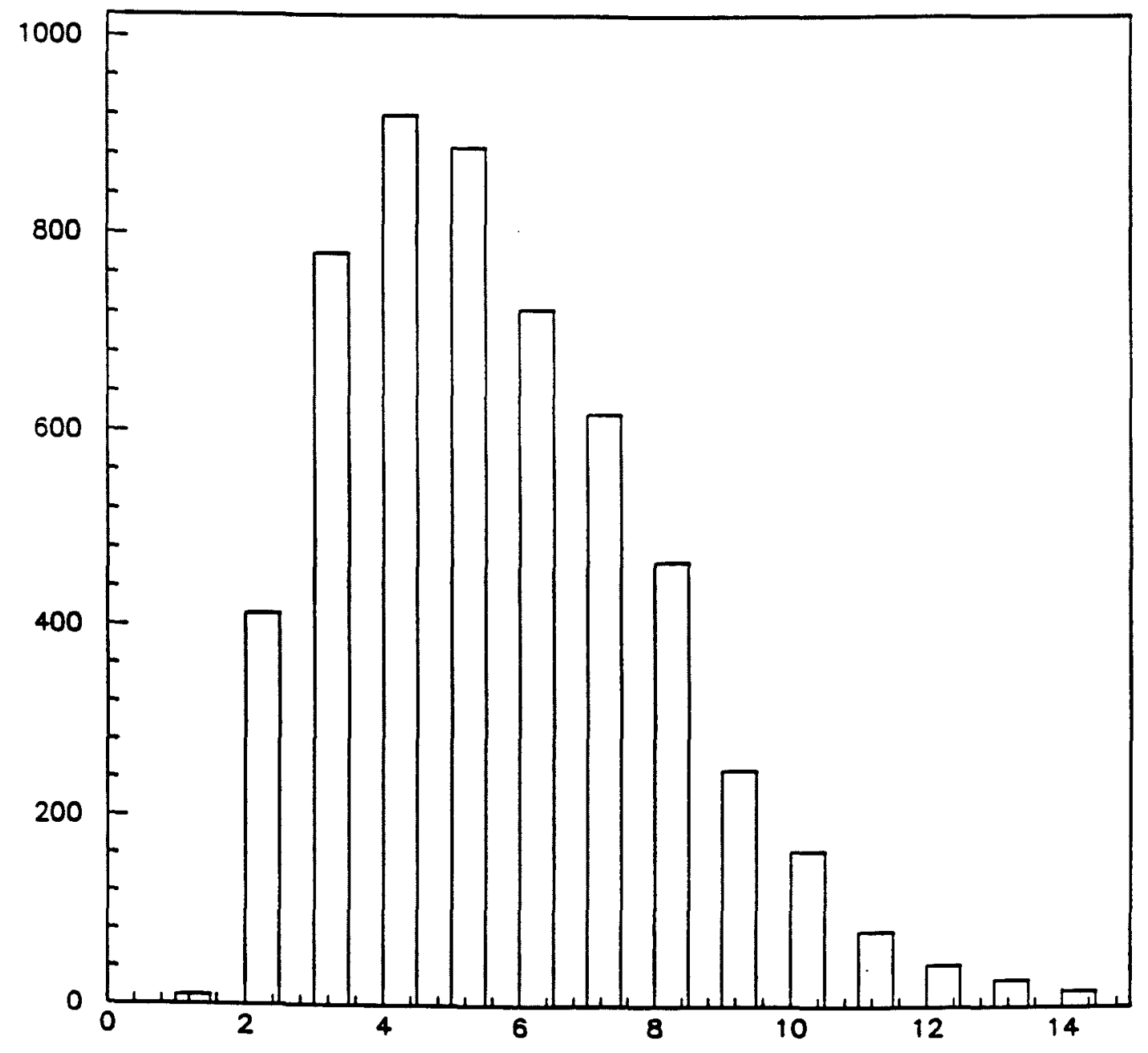

Figure 8.11: recoil jet multiplicity in $\gamma$ events in $\pi^{-}$data 


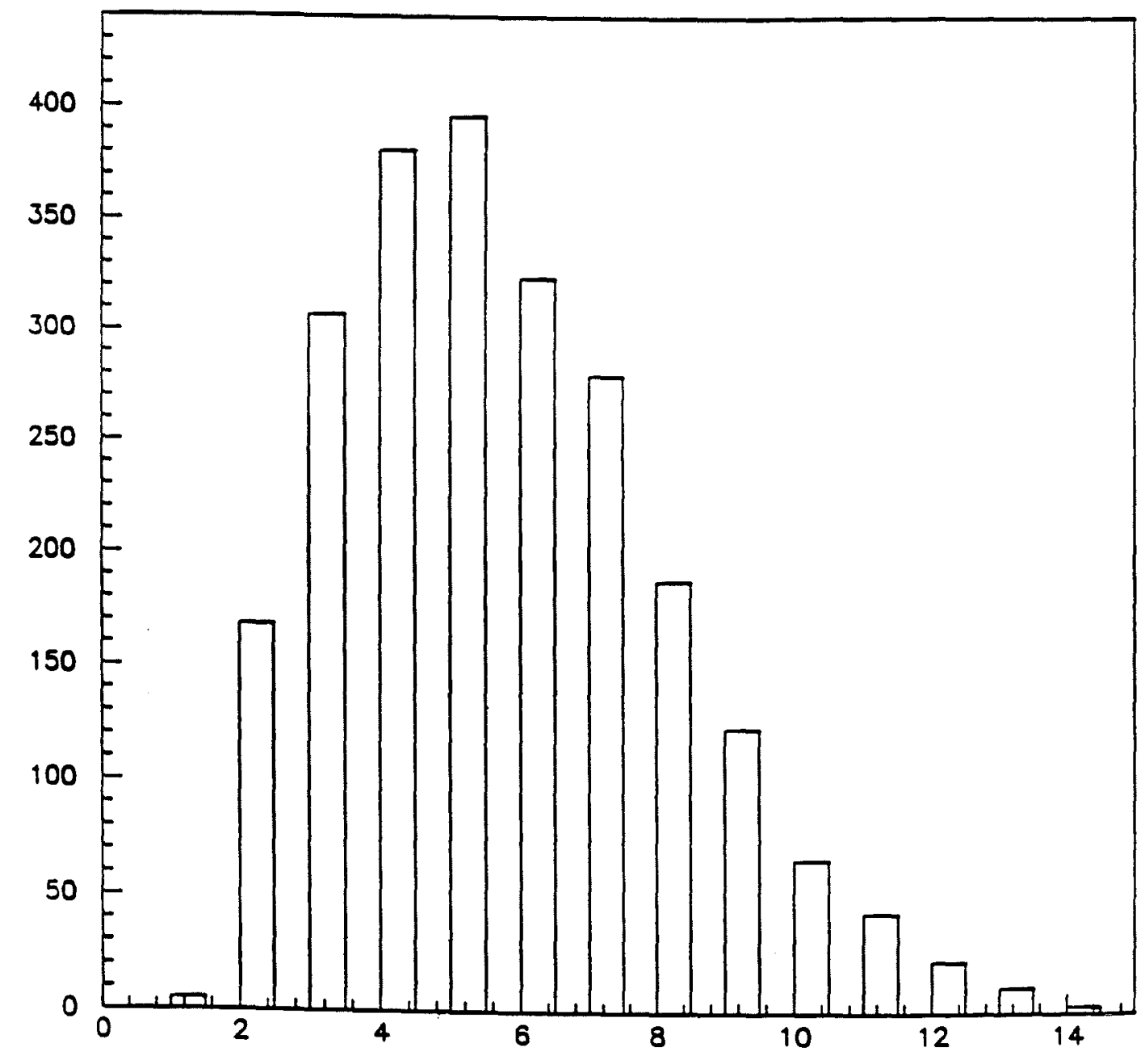

Figure 8.12: recoil jet multiplicity in $\gamma$ events in proton data 


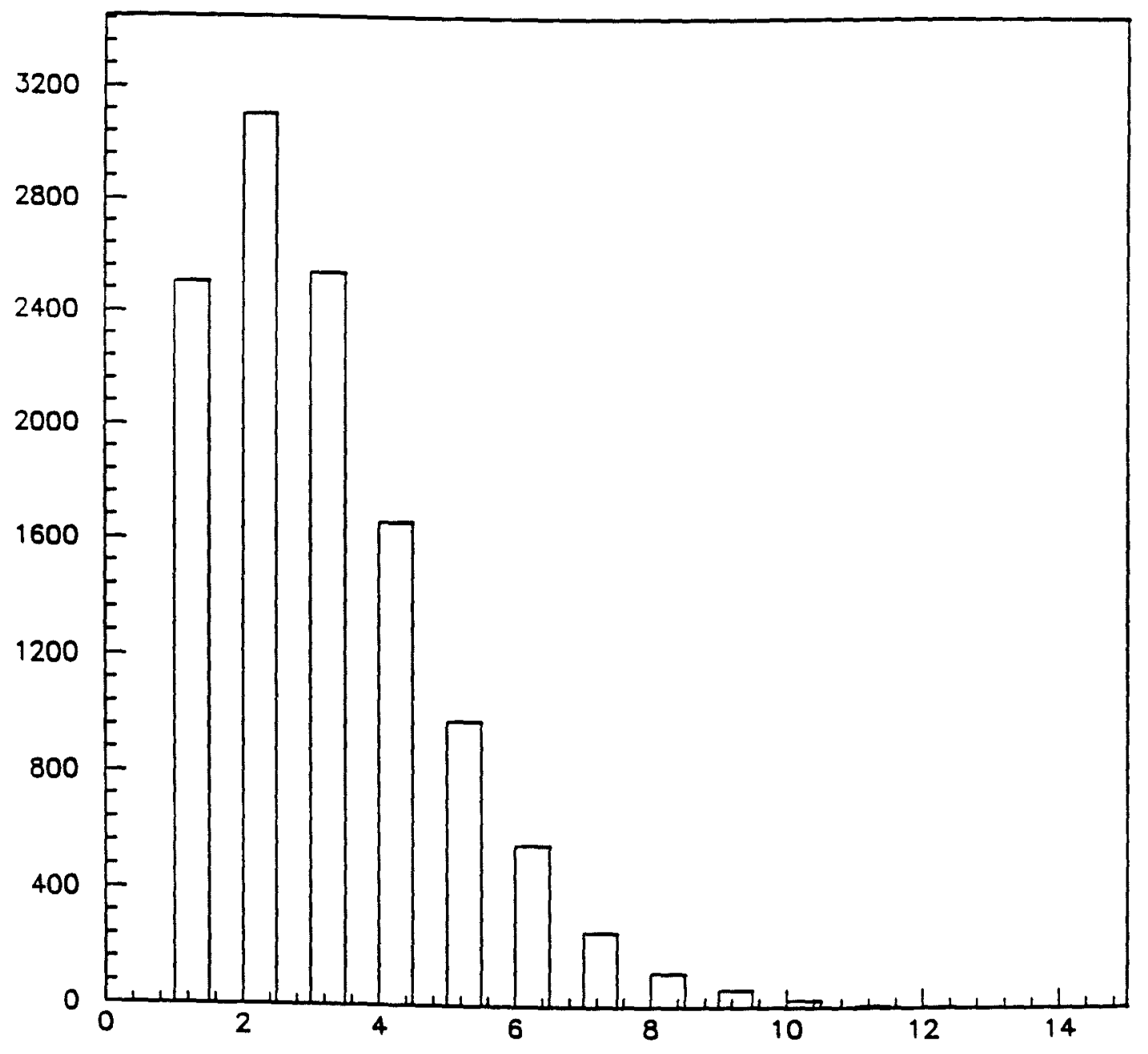

Figure 8.13: Trigger jet multiplicity in $\pi^{0}$ events in $\pi^{-}$data 


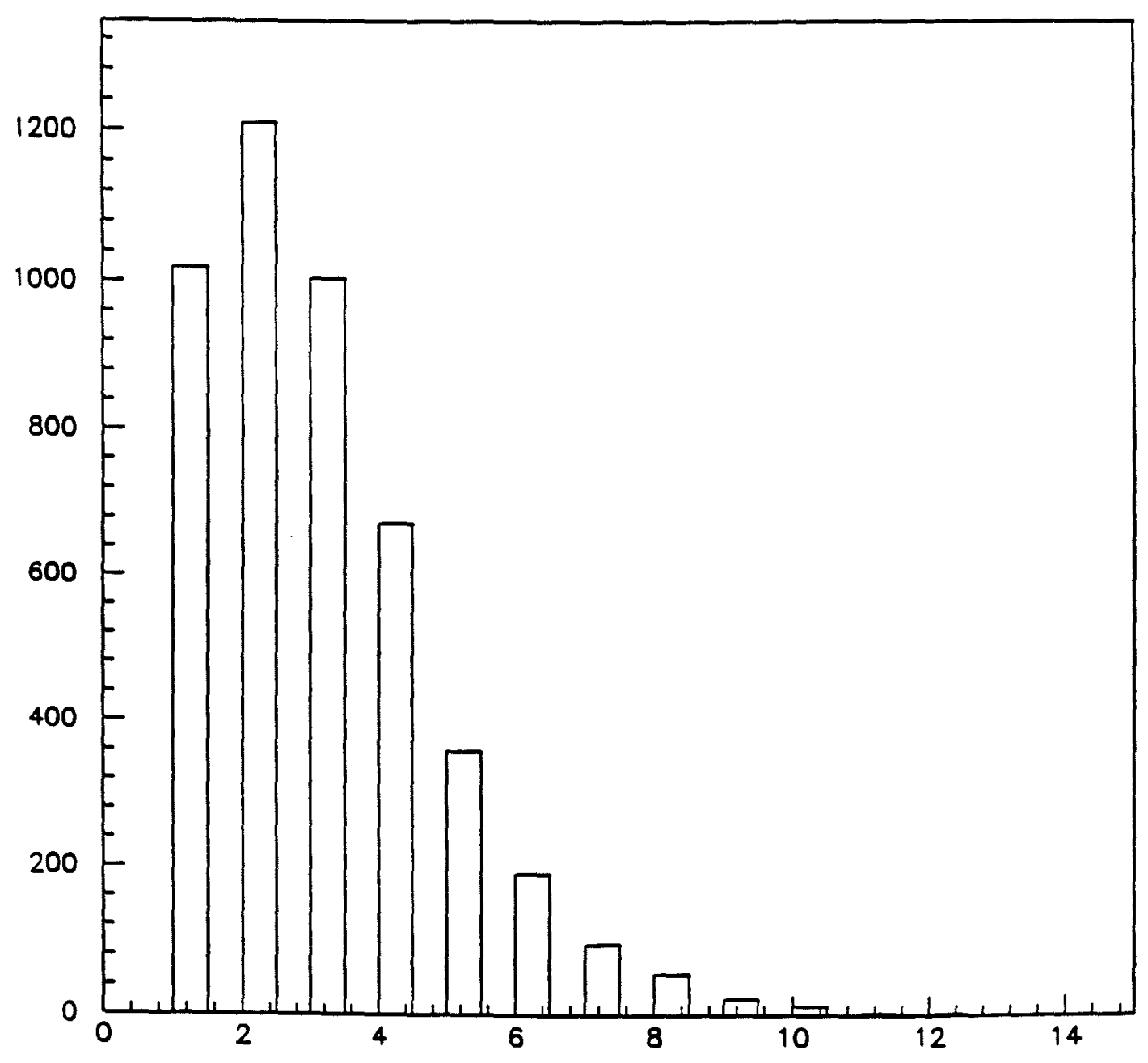

Figure 8.14: Trigger jet multiplicity in $\pi^{0}$ events in proton data 


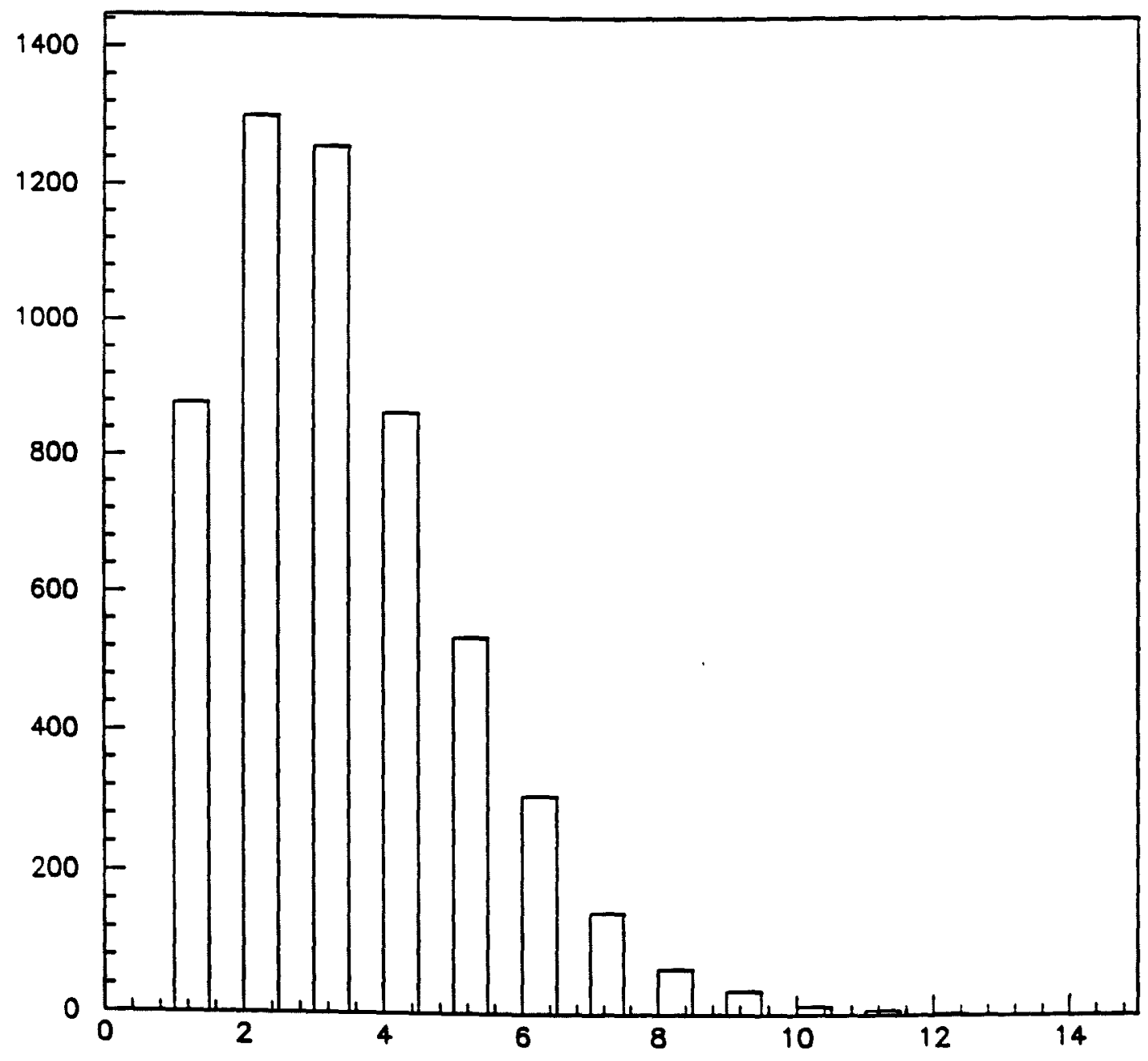

Figure 8.15: Trigger jet multiplicity in $\gamma$ events in $\pi^{-}$data 


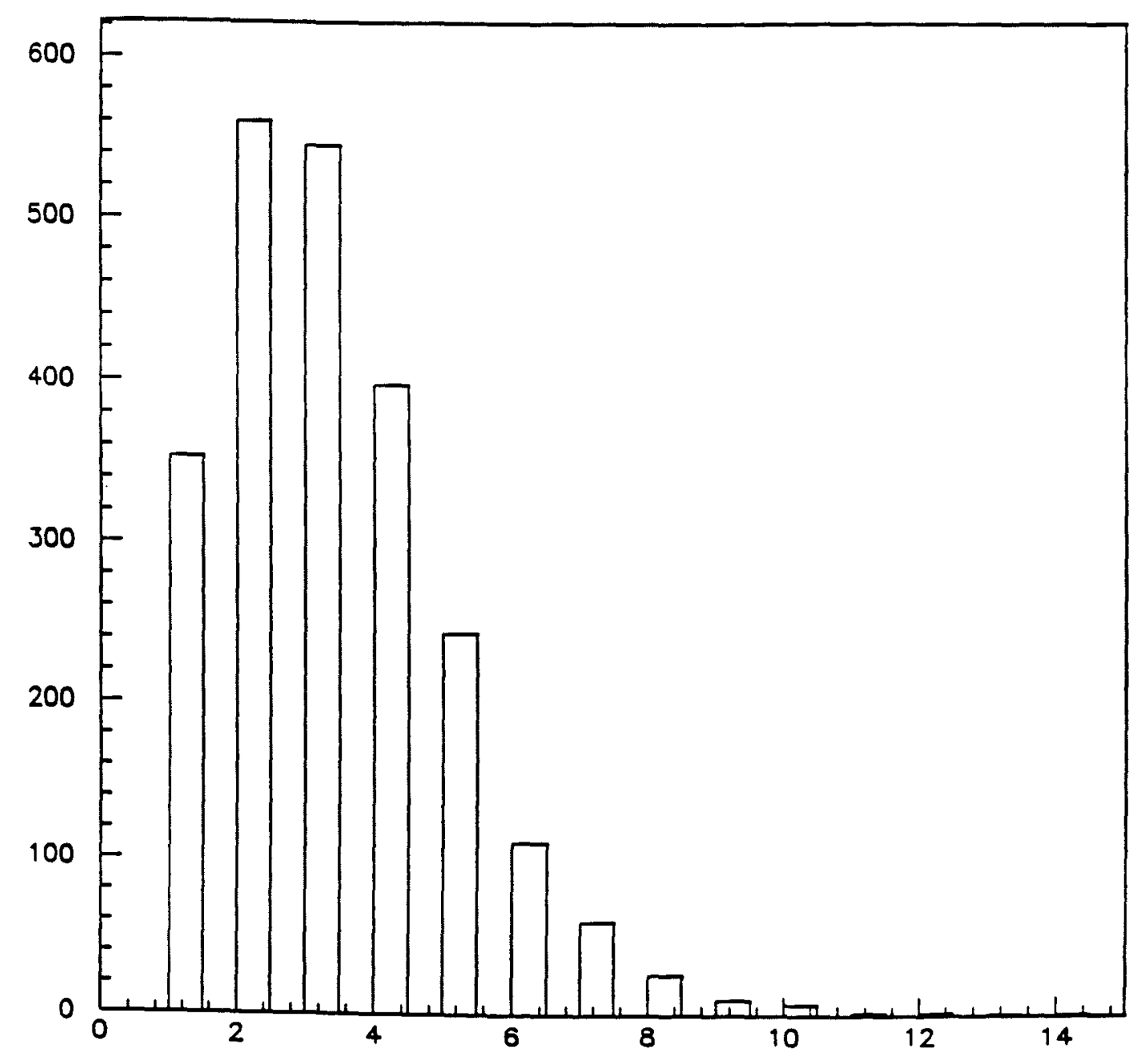

Figure 8.16: Trigger jet multiplicity in $\gamma$ events in proton data 


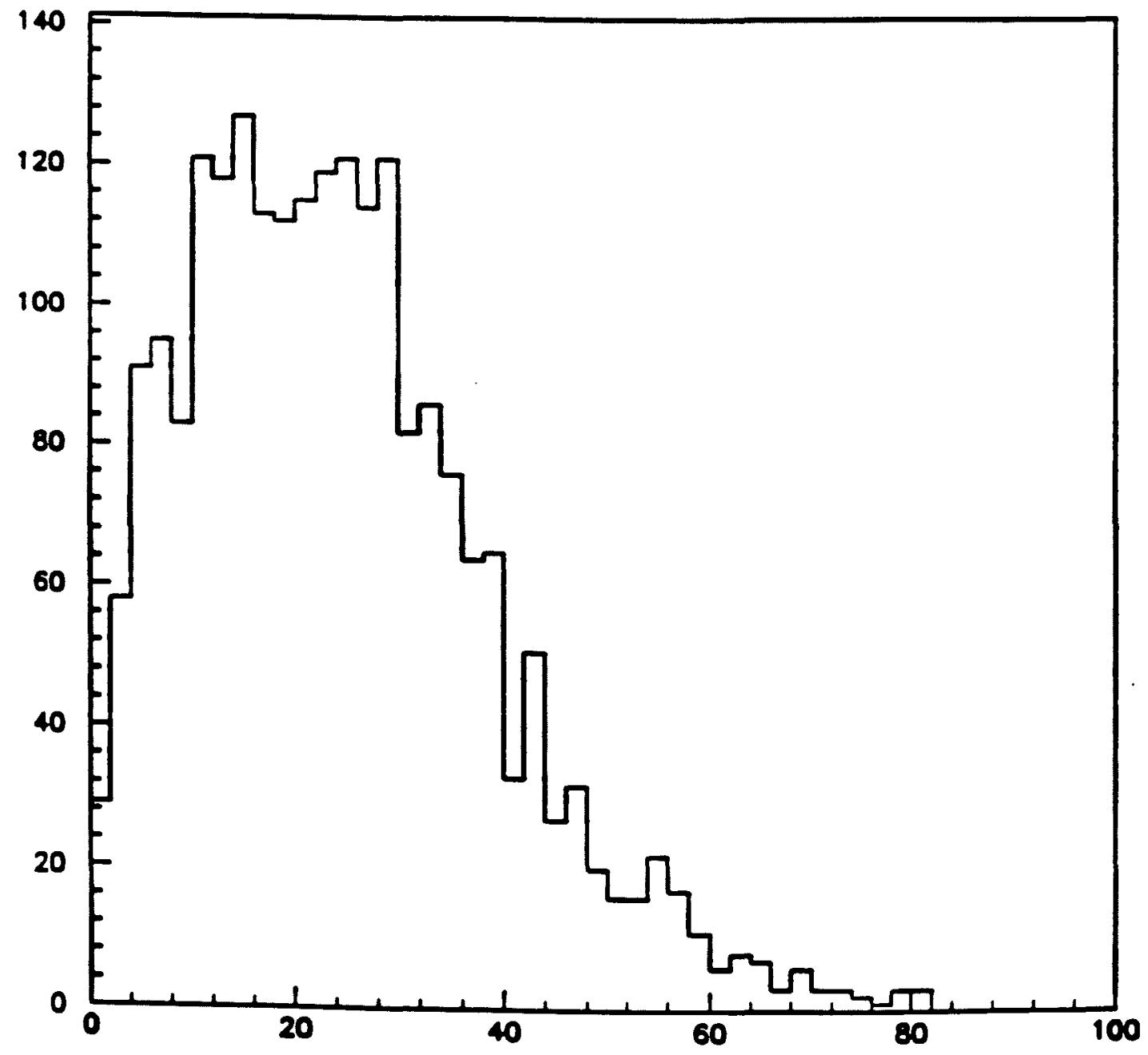

Figure 8.17: Angle of recoil particles with respect to recoil direction 


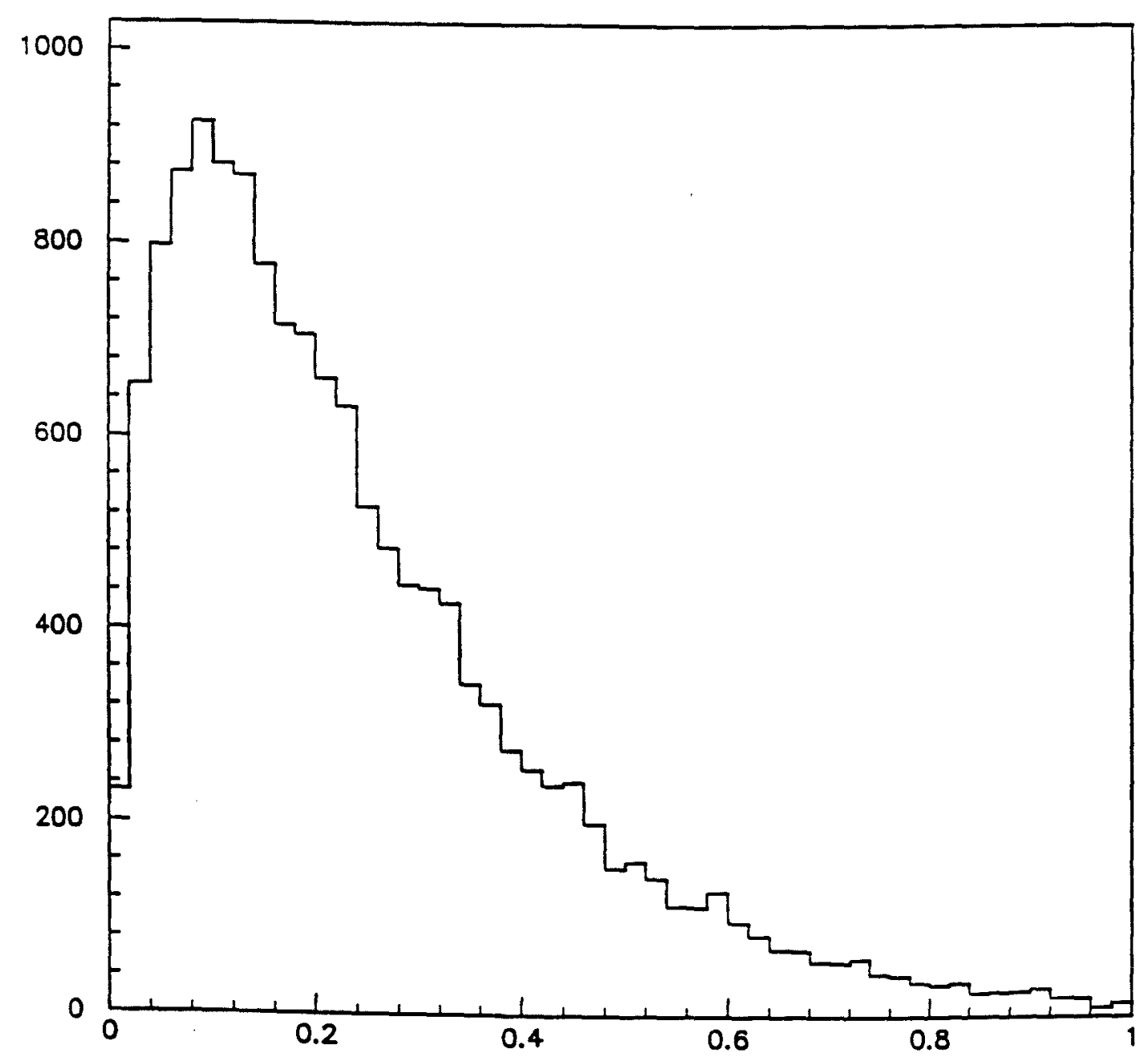

Figure 8.18: Transverse momentum of recoil particles with respect to recoil direction 
Carlo data in proton-proton interactions. The shape of the Monte Carlo distribution seems to be in agreement with E706 data.

The difference between $x_{a}$ and $x_{b}$ is plotted in Figure 8.24 for proton data. This plot has a peak at zero which corresponds to the events where the two resultant parton momenta are equal and opposite to each other.

\subsection{Fragmentation functions}

In the spirit of the hard-scattering model the final state partons hadronise (fragment) into the observable charged pions and $\gamma$ 's. These fragmentation functions are described in terms of $Z_{r}$, the momentum fraction of a particle in a jet. The $Z_{r}$ distribution for recoil jets is plotted in Figure 8.25

and Figure 8.26 for various beam types and for $\gamma\left(\pi^{0}\right)$ trigger particles. These distributions are approximately exponential, with all four distributions essentially identical. In Figure 8.25 a parameterization of a similiar distribution from TASSO [4] data is superimposed. The TASSO data used here is at a center of mass energy of $12 \mathrm{GeV}$ and the parameterization used here is given by:

$$
\frac{1}{N} \frac{d N}{d Z_{r}}=85.0 \exp \left(-38.0 Z_{r}\right)+60.0 \exp \left(-7.9 Z_{r}\right)
$$

where, $N=$ The number of the jet events.

The shape of the two distributions in Figure 8.25 are similiar. In Figure 8.27 the recoil jet $\left(Z_{r}\right)$ is plotted for the proton beam in the Monte Carlo data. 

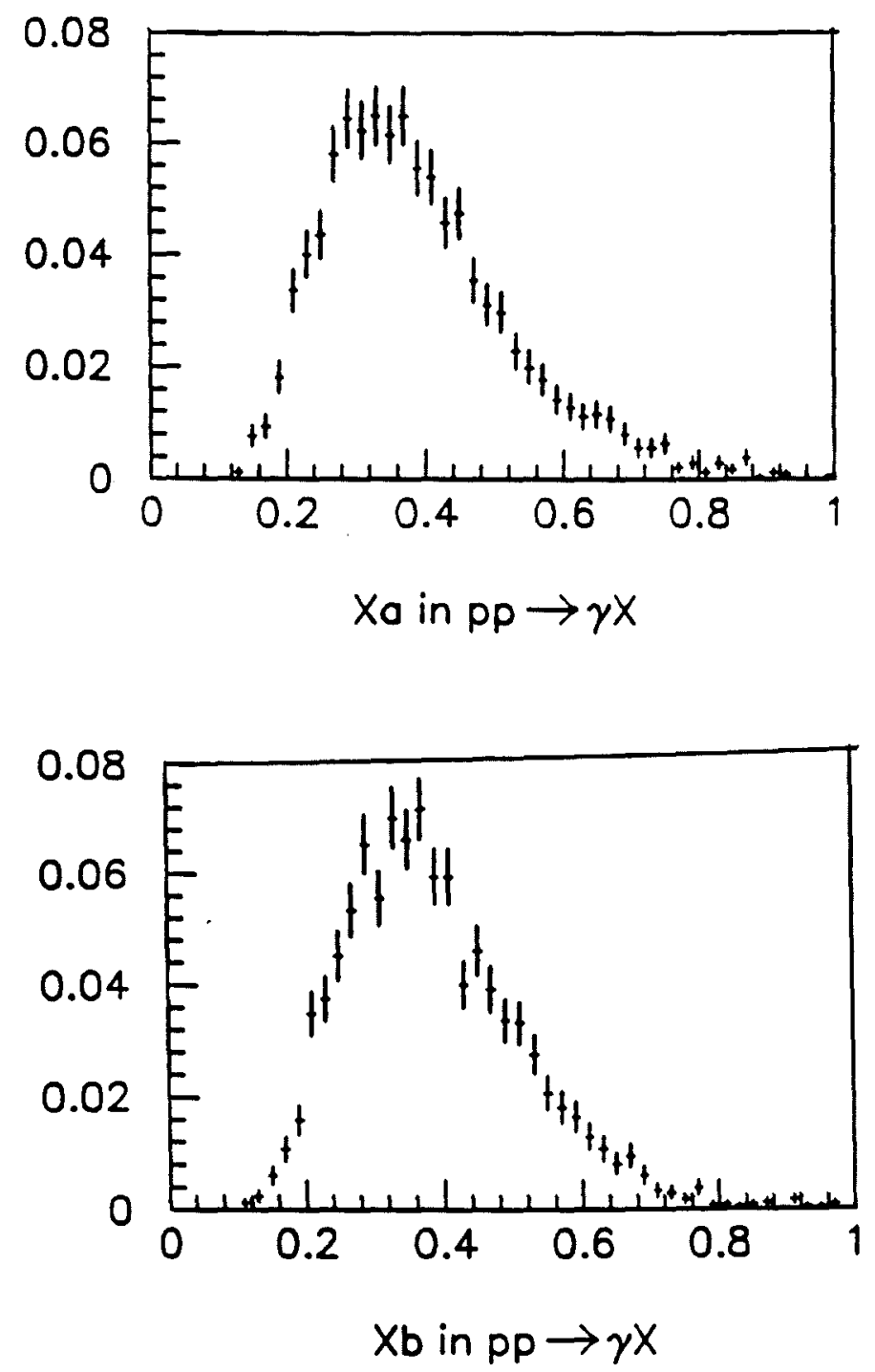

Figure 8.19: $x_{a}$ and $x_{b}$ for $\gamma$ events in proton beam 

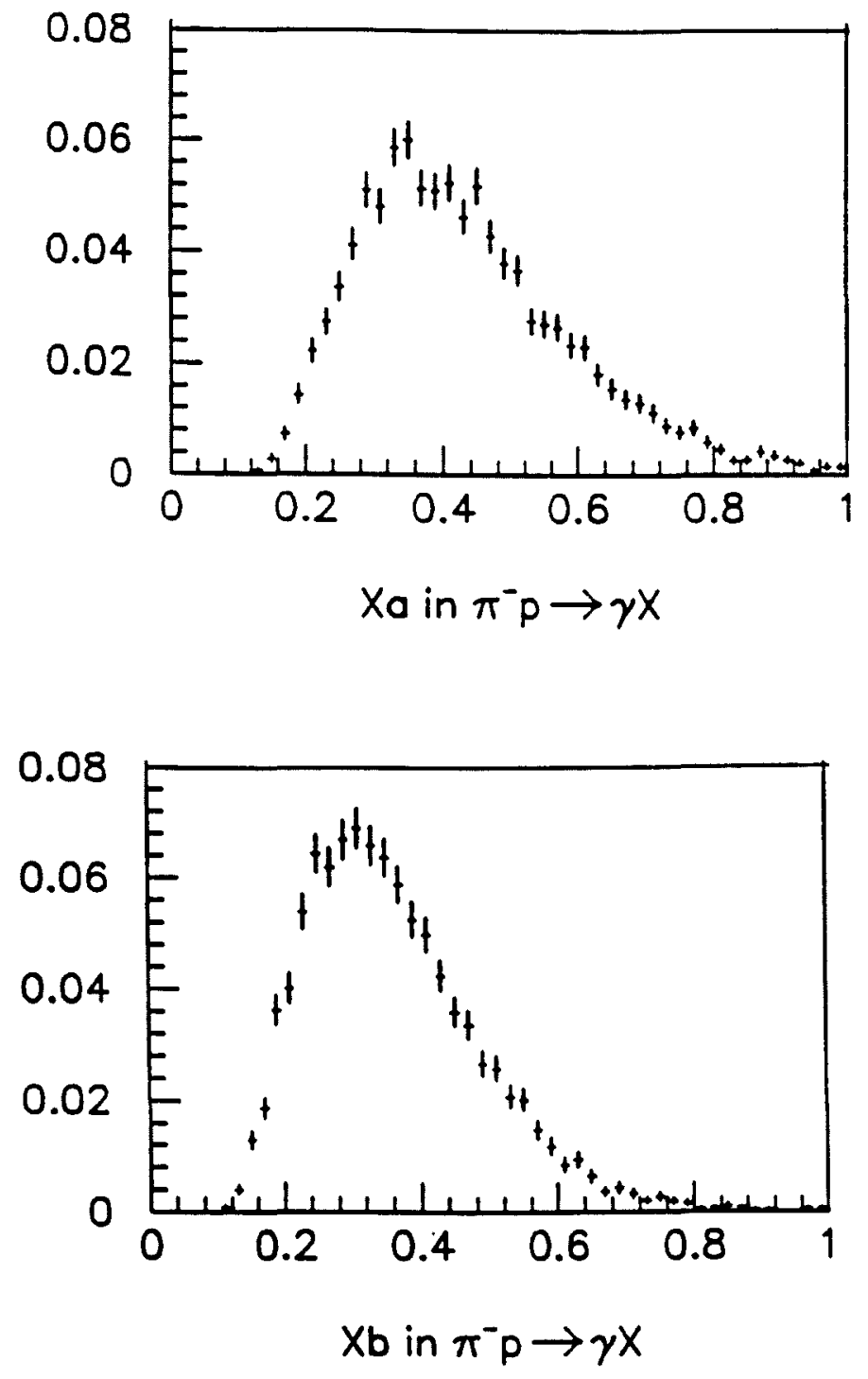

Figure 8.20: $x_{a}$ and $x_{b}$ for $\gamma$ events in $\pi^{-}$beam 

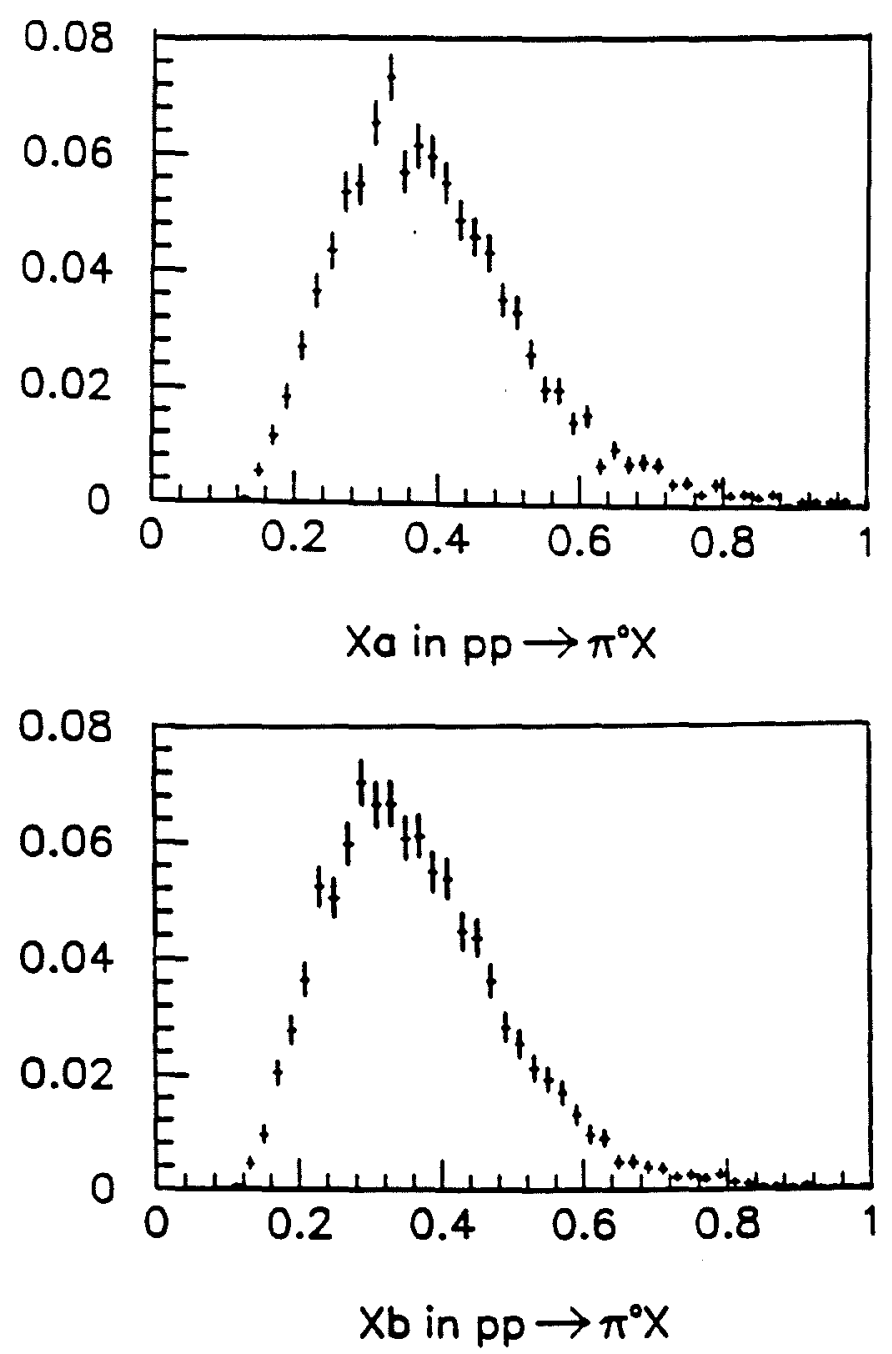

Figure 8.21: $x_{a}$ and $x_{b}$ for $\pi^{0}$ events in proton beam 

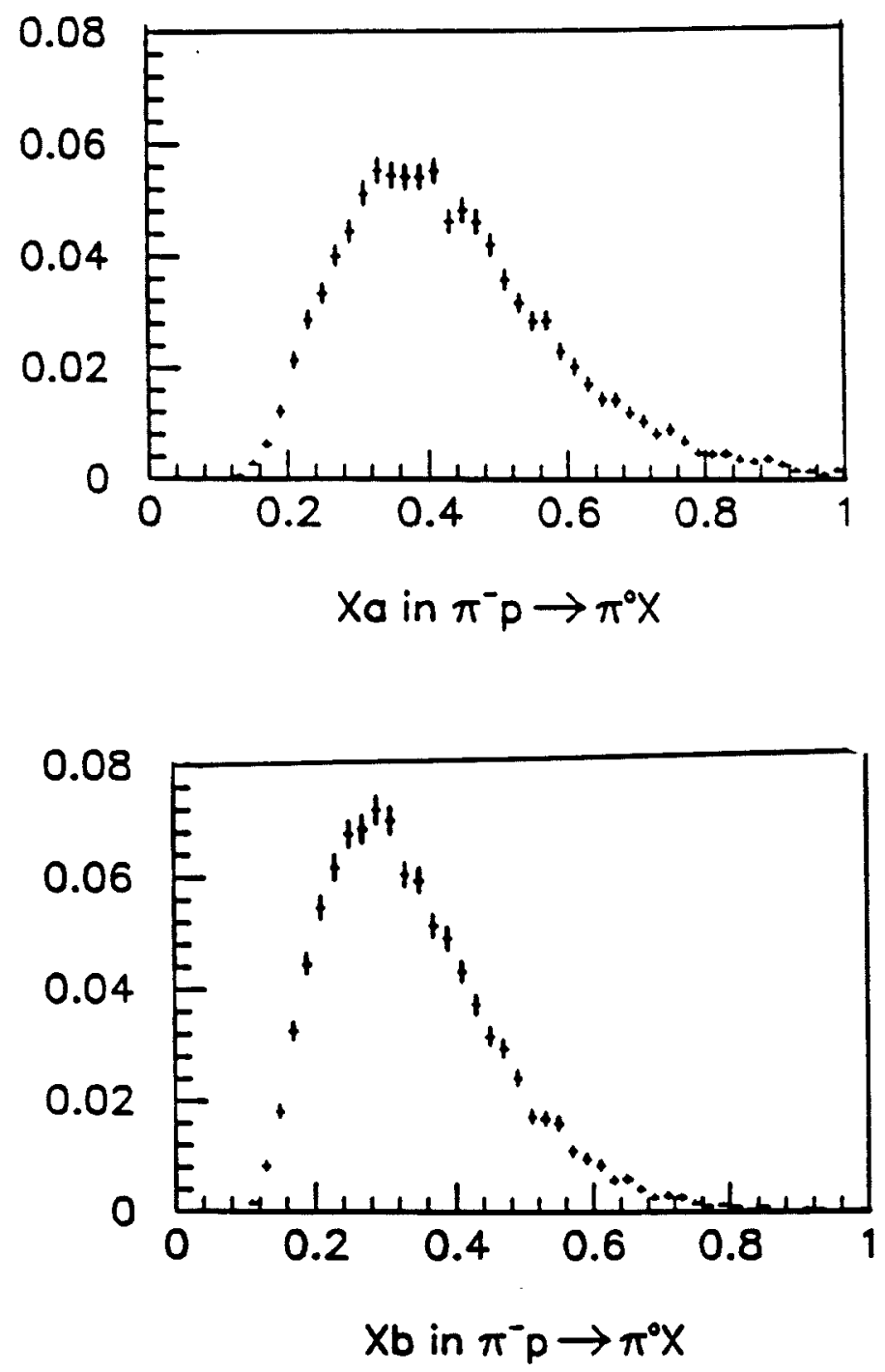

Figure 8.22: $x_{a}$ and $x_{b}$ for $\pi^{0}$ events in $\pi^{-}$beam 

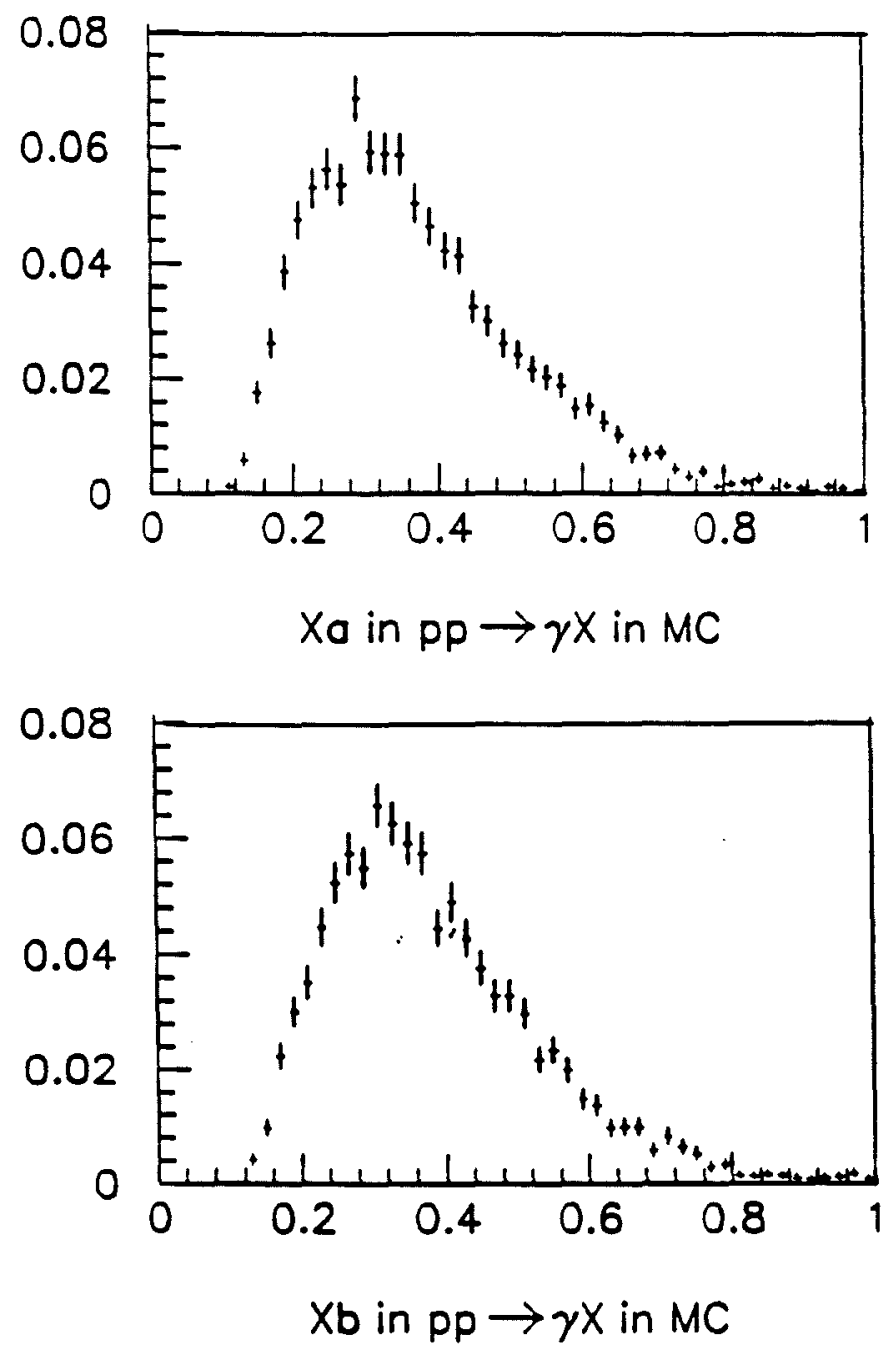

Figure 8.23: $x_{a}$ and $x_{b}$ for $\mathrm{MC}$ events in pp 


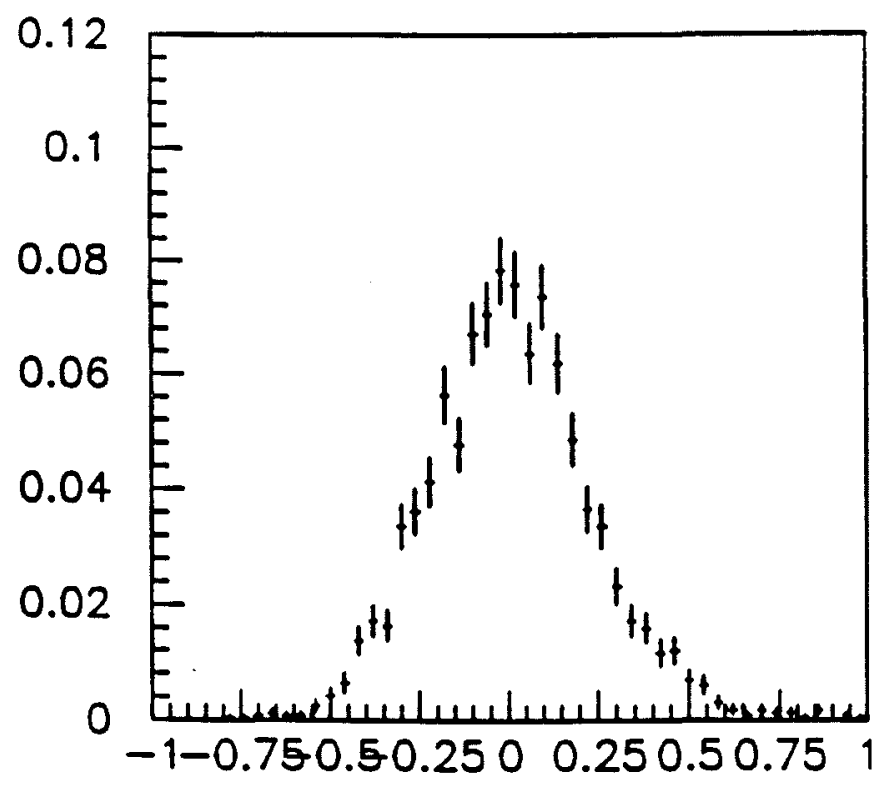

Figure 8.24: Difference between $x_{a}$ and $x_{b}$ for $\gamma$ events in proton beam 
This distribution is similiar to the corresponding one from real data as shown in Figure 8.25(A).

When the $Z_{r}$ variables are plotted for positive and negative particles separately and their ratio taken, the distributions shown in Figure 8.28 are obtained for $\gamma$ events and in Figure 8.29 for the $\pi^{0}$ events. As discussed in Chapter 1 , when the beam sign is negative the process is mainly annihilation and hence the charge ratio is consistent with 1.0. When the beam sign is positive, the Compton process dominates and hence the charge ratio is more than 1. As the $Z_{r}$ of the particles increases, as mentioned in Section 1.2, the particle is more likely to have a positive charge and hence the charge ratio increases. This effect is less pronounced for $\pi^{0}$ events since the recoil particle in this case can be either a quark or a gluon when the beam sign is positive. 

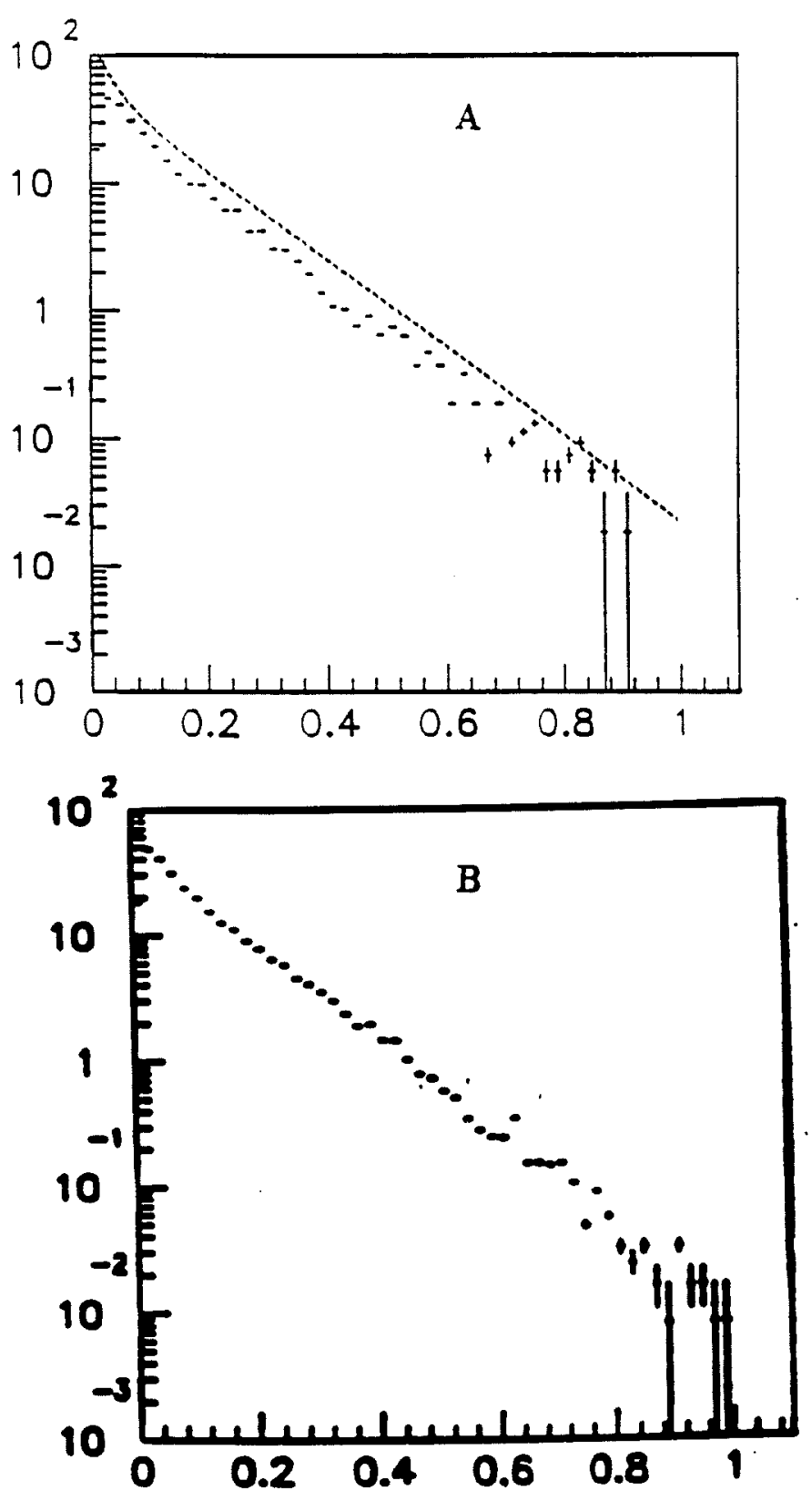

Figure 8.25: Momentum fraction of particles in the recoil jet for single $\gamma$ events in (A) proton data (B) $\pi^{-}$data 

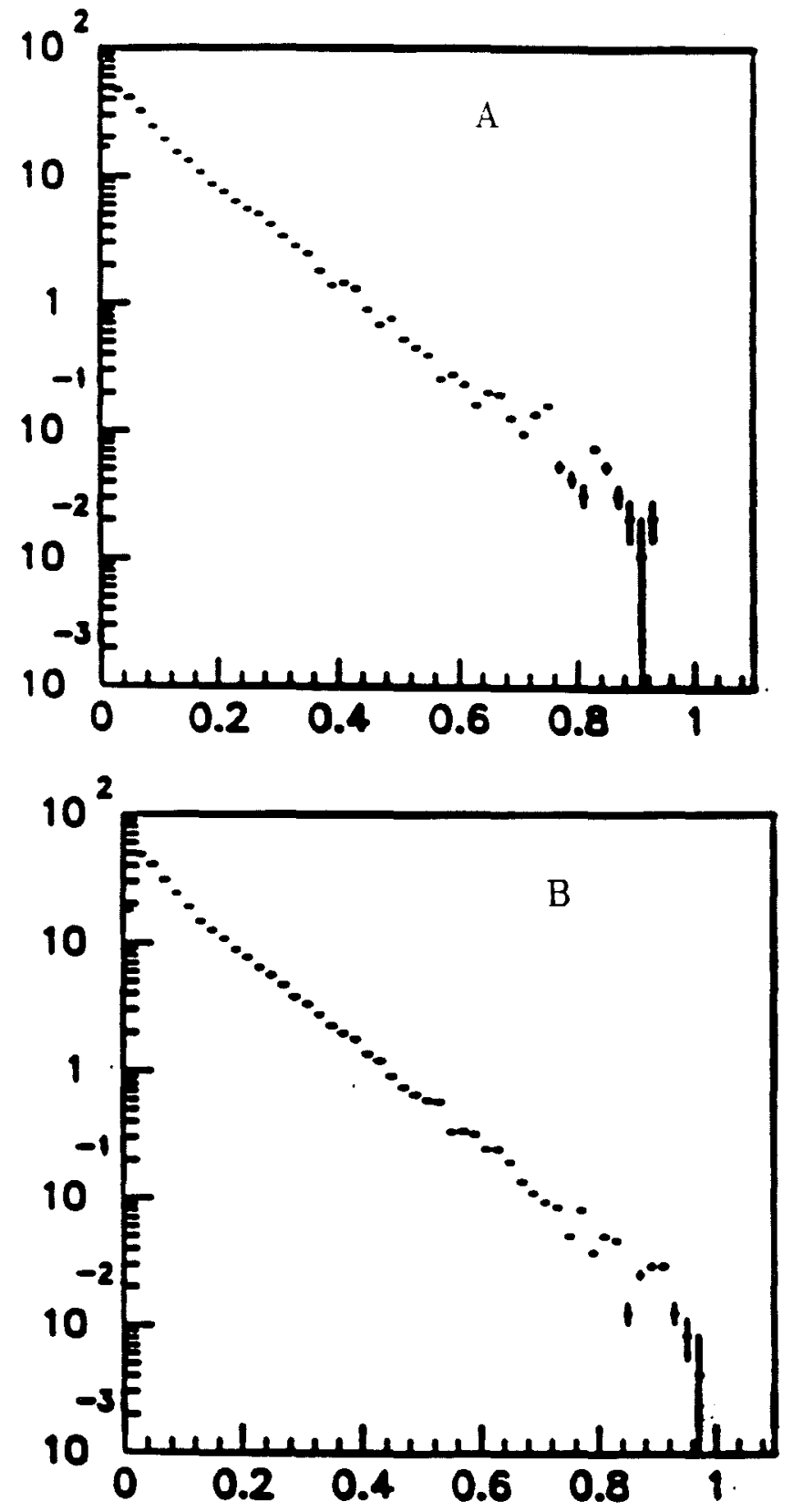

Figure 8.26: Momentum fraction of particies in the recoil jet for $\pi^{0}$ events in (A) proton data (B) $\pi^{-}$data 


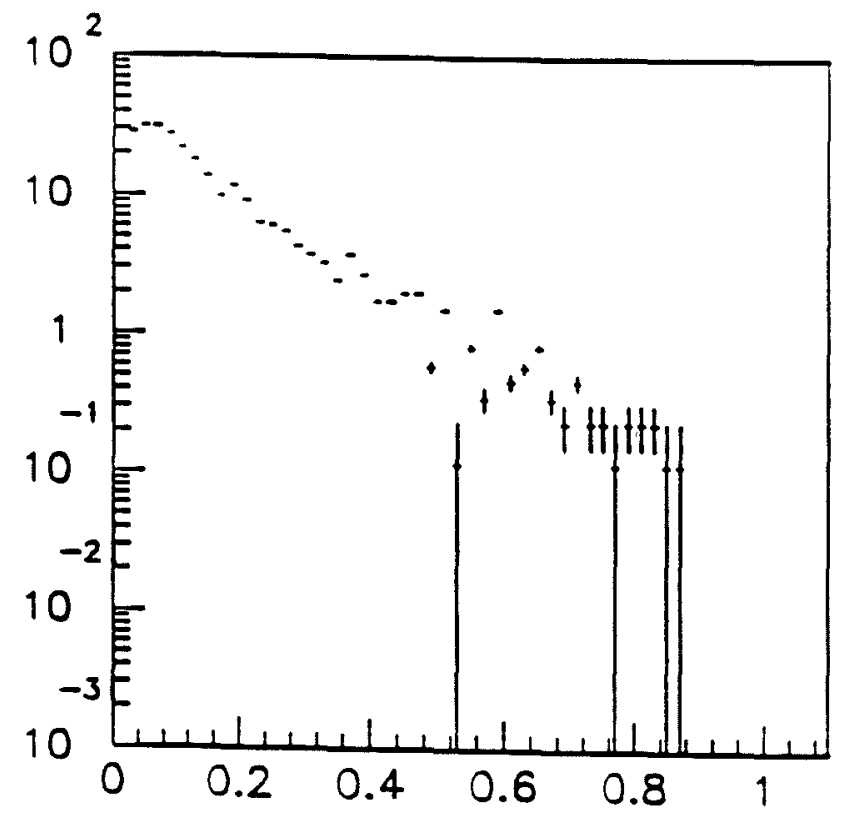

Figure 8.27: Momentum fraction of particles in the recoil jet for proton beam in the Monte Carlo data 

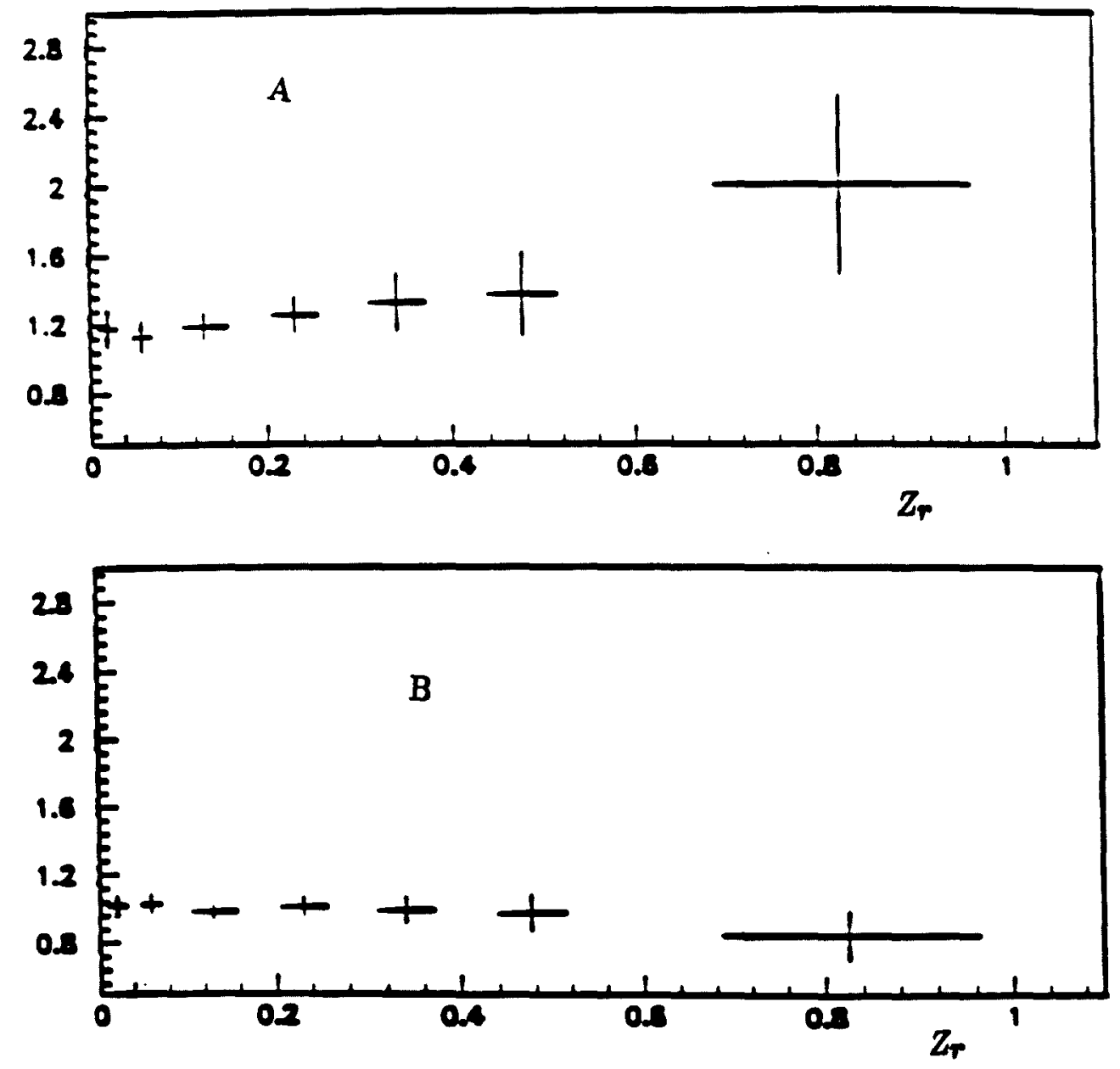

Figure 8.28: Charge ratio in the recoil jet for $\gamma$ events in (A) proton data (B) $\pi^{-}$data 

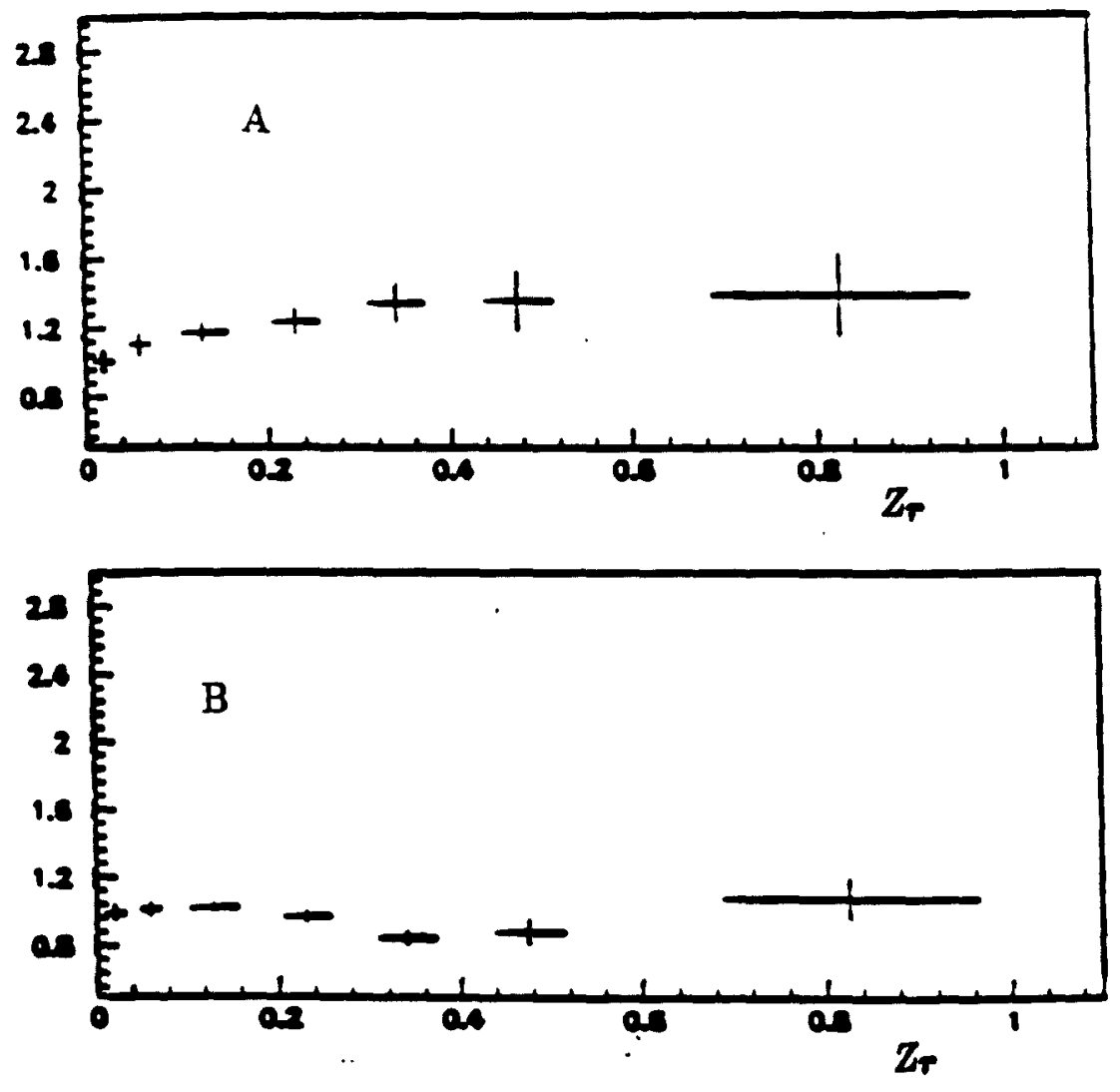

Figure 8.29: Charge ratio in the recoil jet for $\pi^{0}$ events in (A) proton data (B) $\pi^{-}$data 


\section{Chapter 9}

\section{Conclusions}

\subsection{Construction and performance of the apparatus}

Experiment E706 had its first data taking run in 1987-88. Despite the fact that it was the first run of a complicated, newly built spectrometer lots of good data were collected. The operation and the monitoring of the multiwire proportional chambers in particular, and the entire E706 detector in general worked well. The data analyzed in this thesis suggest that many of the systematics of the detector are understood and that the apparatus is able to provide interesting new information about the interactions of partons.

\subsection{Remarks on the analysis of the data}

The electromagnetic calorimeter has observed clean signals of $\pi^{0}$ 's, $\eta$ 's and direct photons. The SSD and MWPC tracking system has been demonstrated to reliably reconstruct the charged particles produced in events triggered by a high $p_{T}$ neutral shower. In addition (not discussed in this thesis) 


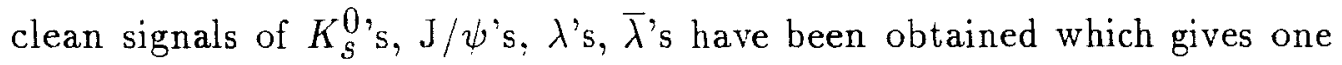
confidence in the momentum calibration of the spectrometer.

From the data collected. two types of events have been identified and studied in this thesis: those which contain (a) $\pi^{0}$ with more than $4 \mathrm{GeV} / \mathrm{c}$ in $p_{T}$ and those which contain a single gamma above $4 \mathrm{GeV} / \mathrm{c}$ in $p_{T}$. The accompanying particles produced in these events show evidence for jet structure. An algorithm to reconstruct the jets has been developed. To check the efficiency of the algorithm Monte Carlo events were generated using structure functions predicted from QCD.These events were passed through the same analysis programs used for the real data. The reconstructed jet directions match well with the generated jet directions giving confidence in the jet finding algorithm. The following properties of the jets have been observed:

- From the events selected with a trigger $p_{T}$ above $4 \mathrm{GeV} / \mathrm{c}$, about $60 \%$ of the single $\gamma$ events and $63 \%$ of the $\pi^{0}$ events have jets.

- The recoil jet direction is dominated by the direction of the leading particle (with the highest $p_{T}$ other than the trigger particle) in the event.

- A measurement of the structure functions of the beam and target partons have been made for both the $\pi^{0}$ events and single $\gamma$ events in the $\pi^{-}$beam data and proton beam data. These functions are most sensitive in the range $0.25-0.50$ of the longitudinal momentum fraction of the partons for a trigger $p_{T}$ above $4 \mathrm{GeV} / \mathrm{c}$. The structure 
functions are in good agreement with Duke-Owens structrue functions as demonstrated by the comparison between the E706 data and the Monte Carlo events generated using the Duke-Owens structure functions.

- The fragmentation functions which describe the hadronisation of partons into charged pions and $\gamma$ 's are exponential and are consistent with the hard-scattering scheme described in Chapter 1. The positive to negative charge ratio also agree with the parton scheme.

The results provided in this thesis have shown the viability of measuring the fragmentation and structure functions from high $p_{T} \pi^{0}$ and $\gamma$ production. In particular when the 1990-91 data taking runs of E706 have acquired an expected factor of 10-15 increase in data, many of these results can be duplicated for various ranges in $\mathrm{M}, x_{a}$ and $x_{b}$, thus providing a more detailed look at the parton structure in high energy collisions. 


\section{Appendix A}

\section{Magnetic Field Parameterization}

In this Appendix it is assumed [15] that the magnetic field does not depend on $\mathrm{x}$ and that there is no field in the $\mathrm{x}$-direction. It is furthermore assumed that there is a "median plane" at $y=0$ in which there is no magnetic field in the z-direction and that:

$$
B_{y}(+y, z)=B_{y}(-y, z)
$$

Then the solutions of Maxwell's equations:

$$
\begin{gathered}
\frac{\partial B_{y}}{\partial z}=\frac{\partial B_{z}}{\partial y} \\
\frac{\partial B_{y}}{\partial y}=-\frac{\partial B_{z}}{\partial z}
\end{gathered}
$$

can be expanded in $\mathrm{y}$ :

$$
\begin{gathered}
B_{y}(y, z)=\sum_{n=0}^{\infty} B_{n}(z) y^{2 n} \\
B_{z}(y, z)=\sum_{n=0}^{\infty} B_{n}^{\prime}(z) \frac{y^{2 n+1}}{2 n+1}
\end{gathered}
$$

yielding:

$$
\sum_{0}^{\infty} B_{n}(z) 2 n y^{2 n-1}=-\sum_{0}^{\infty} B_{n}^{\prime \prime} \frac{y^{2 n+1}}{2 n+1}
$$




$$
B_{n+1}(z) 2(n+1)=-\frac{B_{n}^{\prime \prime}}{2 n+1}
$$

so that:

$$
\begin{gathered}
B_{1}=-\frac{B_{0}^{\prime \prime}}{2} \\
B_{2}=-\frac{B_{1}^{\prime \prime}}{12}=\frac{B_{0}^{\prime \prime \prime \prime}}{24}
\end{gathered}
$$

Similar expressions can be written for $B_{3}, B_{4}$ etc.

Thus knowing $B_{0}(z)=B_{y}(y=0, z)$, one can determine the field everywhere. As long as $B_{y}$ is constant, $B_{z}=0$. As $n$ increases, $B_{n}(z)$ oscillates more and more rapidly. Thus the higher order terms have very little effect on the path of a charged particle going through the magnet:15].

"Zip track" measurements of the field were made at magnetic coil currents of $700 \mathrm{~A}$ and $1400 \mathrm{~A}$. These data were interpolated appropriately and have been corrected for position dependent misalignment of the probes. These measurements were then used to get a field parameterization.

The parameterization is of the form:

$$
B_{0}=\frac{1}{\sum_{0}^{12} a_{n} t^{n}}
$$

where:

$$
t=\sinh \left(\alpha\left(z-z_{0}\right)\right)
$$

The Figure A.1 shows the $B y$ field component and the accompanying Figure A.2 shows the $B_{z}$ field component.

The fringe field is the field created at the edge of the magnet. The dipole field lines are vertical straight lines at regions well within the magnet; at 


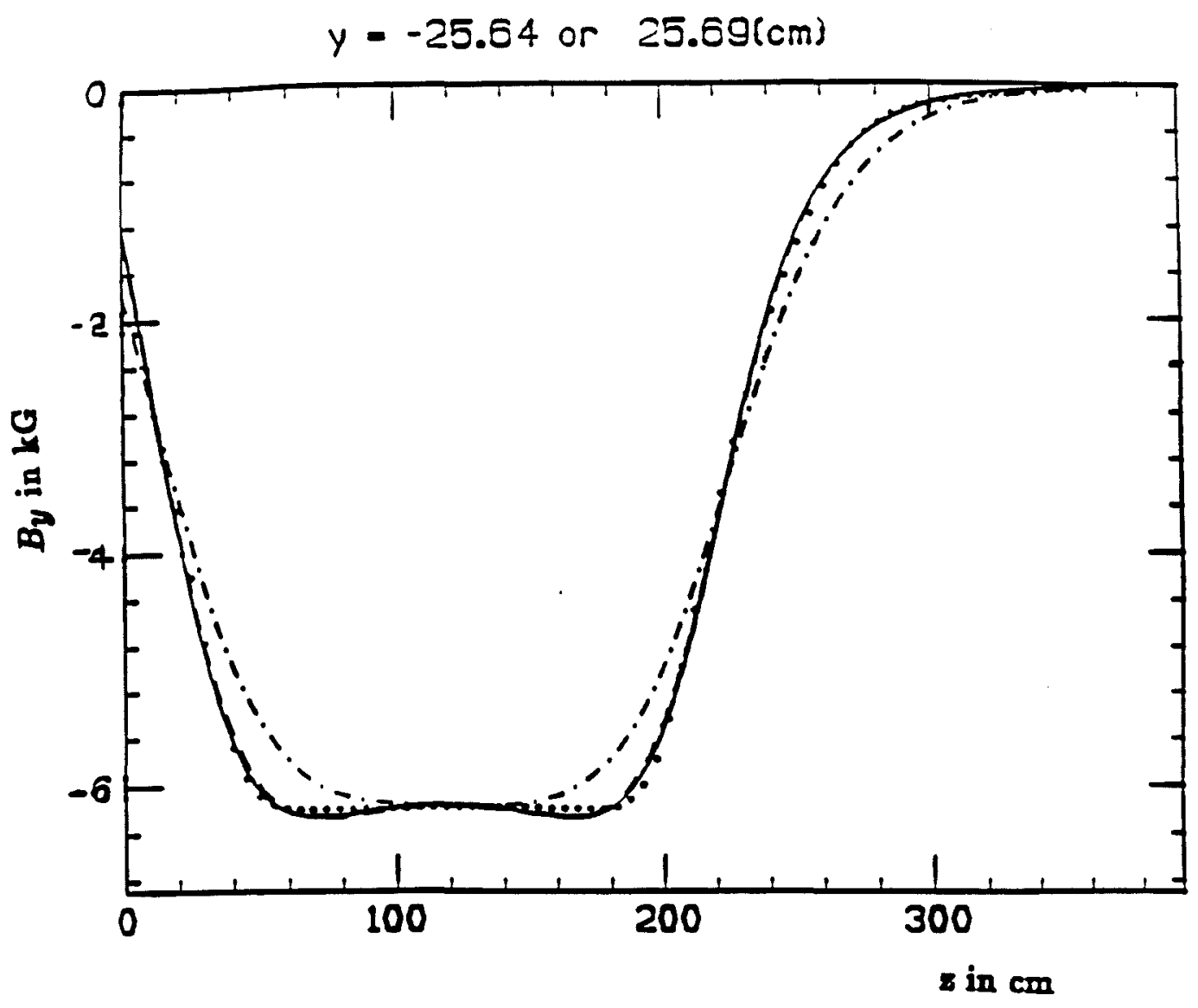

Figure A.1: $B_{y}$ component of the magnetic field versus $\mathrm{z}$ 


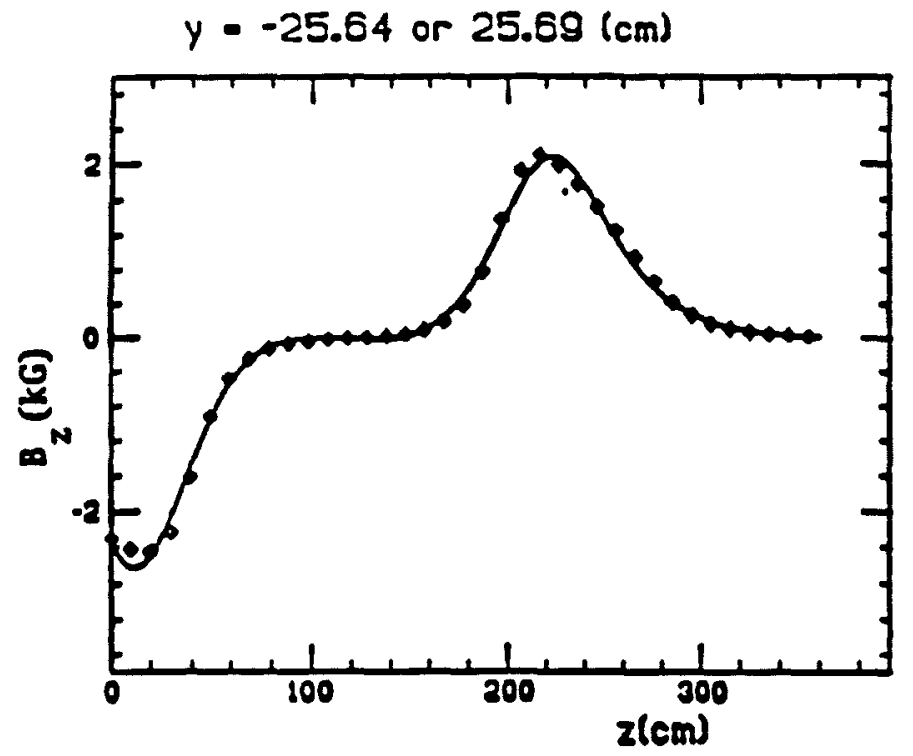

Figure A.2: $B_{z}$ component of the magnetic field versus $z$ 
the edges they curve and give rise to field components in other directions as well.

As can be seen from Figure A.1, the $B_{y}$ component increases and reaches a plateau of about $6.2 \mathrm{kG}$ near the upstream edge of the magnet and remains at this plateau for most parts of the magnet. At the downstream end $B_{y}$ decreases to zero. As shown in Figure A.2, the $B_{z}$ component increases to a maximum of about $3 \mathrm{kG}$ at the upstream and downstream ends of the magnet. This is due to the fringe field at the edges of the magnet. From Figure A.2 it can also be seen that $B_{z}$ is zero for most part of the magnet. The sign of $B_{z}$ is opposite at the two ends of the magnet since the polarity of the fringe field is opposite at these ends. At both ends of the magnet the $B_{x}$ component has a very small value compared to $B_{y}$ and $B_{z}$. This component is also due to the fringe field. $B_{x}$ was measured to be negligible at most parts of the magnet [15].

\section{A.1 Effective field}

Once a fit is obtained, one can replace the magnetic field by an "effective field" $B_{0}$ centered at $z=z_{0}$ and with an "effective length" L. For an optimal fit one can use [15]:

$$
\begin{gathered}
B_{0} L=\int B d z \\
z_{0} B_{0} L=\int z B d z \\
B_{0} L\left(z_{0}^{2}+\frac{L^{2}}{12}\right)=\int z^{2} B d z
\end{gathered}
$$


where the integrals on the right are over the real field. The expressions on the left are the same integrals for a field $B_{0}$ extending from $\left(z_{0}-L / 2\right)$ to $\left(z_{0}+L / 2\right)$.

Using these fits one obtains:

$$
\begin{gathered}
Z_{0}=197.2 \mathrm{~cm} \\
B_{0} L=0.3973 \mathrm{GeV} / \mathrm{c} \\
L=241.6 \mathrm{~cm} \\
B_{0}=5.488 \mathrm{kG}
\end{gathered}
$$




\section{Appendix B}

\section{Physics Principles of MWPC's}

\section{B.1 Interaction of a charged particle in a gaseous medium}

A fast charged particle travelling in a gaseous medium can interact with it in many ways $[10,27]$. Of these the electromagnetic ones are more common. The incoherent Coulomb interactions between the electromagnetic fields of the incoming charged particle and of the medium result in excitation and ionization of the atoms of the medium. These form the basis for the detection of charged particles. For particles heavier than electrons, the contributions of other electromagnetic processes, such as bremsstrahlung, Cerenkov and transition radiation, to the total energy loss of the incoming particle is negligible in gaseous detectors. 


\section{B.2 Geometry of MWPC}

A multiwire proportional chamber (MWPC) [27! consists of a set of planes of thin, parallel, equally spaced wires and a set of planes made of conductive material. The wires are called anode wires and the planes containing them are called anode planes. The conducting planes are called cathode planes. Each of the anode planes is sandwiched between two cathode planes. For proper operation of this system, the distance between adjacent planes is normally three or four times larger than the distance between the wires in the anode plane. An appropriate gas mixture flows in between this assembly of planes.

\section{B.3 Electrostatics of MWPC}

When a negative potential is applied to the cathodes and the anodes are kept at ground potential, an electric field develops between them as indicated in Figure B.1 which shows the electric field equipotentials around the anode planes along a direction perpendicular to the anode planes. The potential (V) and the electric field (E) in the region between the planes can be expressed as [27]:

$$
\begin{gathered}
V(x, y)=\frac{C V_{0}}{4 \pi \epsilon_{0}}\left[\frac{2 \pi l}{s}-\ln \left(4\left(\sin ^{2}\left(\frac{\pi x}{s}\right)+\sinh ^{2}\left(\frac{\pi y}{s}\right)\right)\right)\right] \\
E(x, y)=\frac{C V_{0}}{2 \epsilon_{0} s}\left[1+\tan ^{2}\left(\frac{\pi x}{s}\right) \tanh ^{2}\left(\frac{\pi y}{s}\right)\right]^{\frac{1}{2}}\left[\tan ^{2}\left(\frac{\pi x}{s}\right)+\tanh ^{2}\left(\frac{\pi y}{s}\right)\right]^{-\frac{1}{2}} \\
C=\frac{2 \pi \epsilon_{0}}{(\pi l / s)-\ln ((2 \pi a) / s)}
\end{gathered}
$$




$$
\begin{aligned}
& E_{y}=E(0, y)=\frac{C V_{0}}{2 \epsilon_{0} s} \operatorname{coth}\left(\frac{\pi y}{s}\right) \\
& E_{x}=E(x, 0)=\frac{C V_{0}}{2 \epsilon_{0} s} \cot \left(\frac{\pi x}{s}\right)
\end{aligned}
$$

For $y \ll s:$

$$
E(x, y) \simeq \frac{C V_{0}}{2 \pi \epsilon_{0}} \frac{1}{r}
$$

where:

$$
r=\sqrt{x^{2}+y^{2}}
$$

For $y \gg s:$

$$
\begin{gathered}
\operatorname{coth}\left(\frac{\pi y}{s}\right) \simeq 1 \\
E_{y}=\frac{C V_{0}}{2 \epsilon_{0} s}
\end{gathered}
$$

where:

$\mathrm{C}=$ pacitance per unit length

$\mathrm{a}=$ anode wire radius,

$l=$ distance between planes, and

$s=$ wire spacing in the anode plane.

Very close to the anode $(y \ll s)$ :

$$
E=\frac{C V_{0}}{2 \pi \epsilon_{0} r}
$$

As a result, around the anode wire the field is radial. This is illustrated in Figure B.1.

If one of the wires were to be slightly displaced mechanically from the plane of the anode, it would be more attracted to the side of the displacement. Only the mechanical tension on the wire would constrain this motion 
due to attraction $[30]$. In equilibrium, this can create a situation in which the wires are displaced alternately up and down as shown in Figure B.2.

The force per unit length between two parallel linear charges $C V_{0}$ a distance $r$ apart is [30]:

$$
F=\frac{\left(C V_{0}\right)^{2}}{2 \pi \epsilon_{0}} \frac{1}{r}
$$

and its component in the direction perpendicular to the wire plane is:

$$
F_{\perp}=\frac{\left(C V_{0}\right)^{2} \pi \delta}{4 \epsilon_{0} s^{2}}
$$

where $\delta(x)$ is the displacement of the wire from the plane of the anode as shown in Figure B.2. If $\mathrm{T}$ is the mechanical tension on the wire, the restoring force per unit length in the direction perpendicular to the wire plane is:

$$
R=T \frac{d^{2} \delta}{d x^{2}}
$$

where $\mathrm{x}$ is the dimension along the wire. If $\mathrm{L}$ is the length of the wire

$$
\delta(0)=\delta(L)=0
$$

For equilibrium one can equate the above equations (B.12) and (B.13):

$$
T \frac{d^{2} \delta}{d x^{2}}=-\frac{\left(C V_{0}\right)^{2}}{4 \epsilon_{0}} \pi \frac{\delta}{s^{2}}
$$

Solving this equation, one finds:

$$
\delta(x)=\delta_{0} \sin \left(\frac{C V_{0}^{\gamma}}{2 s} \sqrt{\frac{\pi}{\epsilon_{0} T}} x\right)
$$

Let:

$$
T_{c}=\frac{1}{4 \pi \epsilon_{0}}\left(\frac{C V_{0} L}{s}\right)^{2}
$$



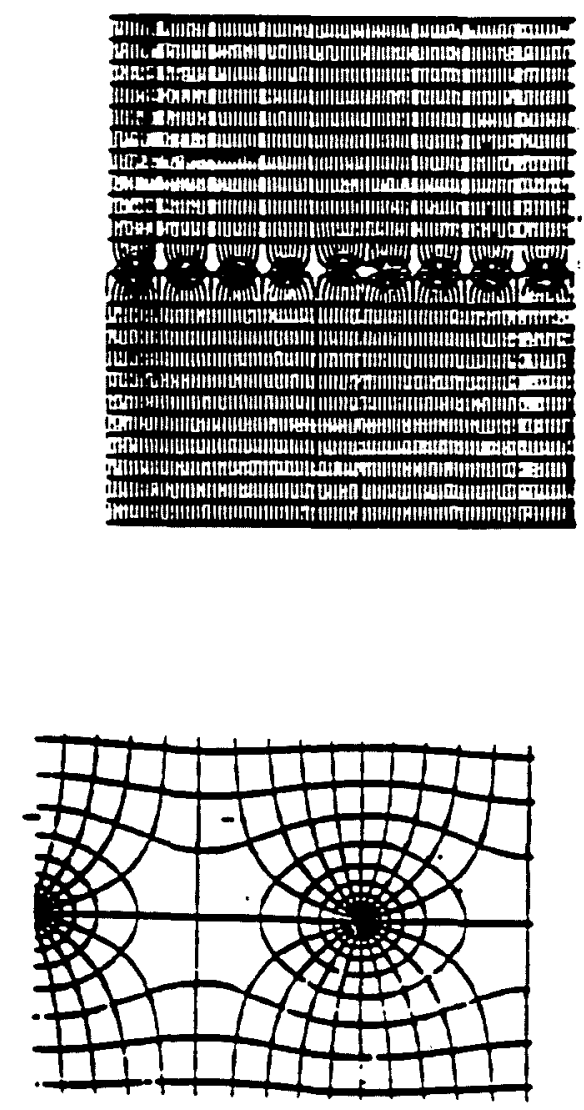

Figure B.1: Electric field equipotential lines inside the MWPC 


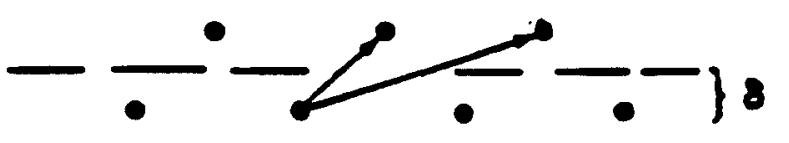

Figure B.2: Effect of mechanical displacement of wires 
Then the condition for stability is:

$$
T>T_{C}
$$

For a typical MWPC used in E706, one has:

$\mathrm{a}=1.016 \times 10^{-3} \mathrm{~cm}$

$1=0.574 \mathrm{~cm}$

$\mathrm{s}=0.254 \mathrm{~cm}$

$\mathrm{L}=200 \mathrm{~cm}$

$\mathrm{V}_{0}=2900 \mathrm{~V}$

$\mathrm{T}=40 \mathrm{~g}$ weight $=3.9 \times 10^{-3} \mathrm{~N}$.

For this configuration, using the above equations:

$\mathrm{C}=14.4 \times 10^{-12} \mathrm{Fm}^{-1}$

$T_{c}=8.0 \times 10^{-5} \mathrm{~N}$.

Here $T>T_{c}$ and hence the stability conditions are met in this configuration.

For a given tension allowed by the elasticity of the wire, the minimum length is:

$$
L_{c}=\frac{s}{C V_{0}} \sqrt{4 \pi \epsilon_{0} T_{m}}
$$

Another consequence of the electrostatic forces is the overall attraction of the cathode planes towards the anode plane. This can cause an inflection of the cathode planes with a reduction of the gap width between the planes. As shown in reference [27], the electric field at the surface of the cathode is:

$$
E_{s}=\frac{E_{y}}{2}=\frac{C V_{0}}{4 \epsilon_{0} s}
$$

so that the average charge per unit surface area on each cathode is $C V_{0}^{\prime} / 2 s$. 
For a typical cathode used in E706:

$E_{s}=375.49 \mathrm{~V} / \mathrm{m}$

Assuming that the field and charge distribution on the surface of the cathodes is a constant, the electrostatic pressure on each cathode is:

$$
P=\frac{C^{2} V_{0}^{2}}{8 \epsilon_{0} s^{2}}
$$

For a typical cathode used in $\mathrm{E} 706, \mathrm{P}=3.8 \mathrm{~N}$. The maximum inward deflection of the cathode of surface area $H^{2}$ stretched with a linear tension of $\mathrm{T}$ and subject to a pressure $\mathrm{P}$ is given by

$$
\Delta Y=\frac{P H^{2}}{8 T}
$$

This sets a maximum for the size for the chamber. In large chambers such as the one used in this experiment, an additional mechanical spacer is used to keep the planes separated. For a typical cathode used in E706, for a tension $\mathrm{T}^{\prime}=210^{3} \mathrm{~N} \mathrm{~m}^{-1}, \Delta Y=0.95 \mathrm{~mm}$.

Mechanical spacers are used in the MWPC's used in E706 in order to avoid this deflection.

\section{B.4 Dynamics of MWPC}

When a charged particle goes through a MWPC, additional charges are produced by the interaction of the particle with the gaseous medium. In the region where charges are produced, the electric field makes electrons drift towards the anode and the positive ions drift towards the cathode. But closer to anode, the field gets strong enough to start charge multiplication. 
The electrons are collected on the anode in a very short time and a cloud of positive ions starts to migrate towards the cathode. This process happens when the applied operating voltage $\left(V_{0}\right)$ between anode and cathode is above a certain threshold voltage $\left(V_{T}\right)$, characteristic of the system.

The charge collected at the anode is proportional to the original charge deposited for $V_{0}$ moderately above $V_{T}$, through a proportionality constant called the multiplication factor $(M)$. The amount of charge collected per input charge is called the gain of the MWPC and will depend upon the details of the geometry of the chamber. Figure B.3 shows how the multiplication factor $M$ varies as a function of $V_{0} / V_{T}$. As the thickness of the anode wire increases, the curve gets steeper. If a wire has non-uniform thickness then the value of $M$ is different at different parts of the wire. Hence the plot of $V_{0} / V_{T}$ versus $\mathrm{M}$ for such wires would be thick bands instead of thin straight lines. If $\mathrm{M}$ is different in different regions of the chamber, one section of the chamber would begin to discharge when another section is not yet properly amplifying.

\section{B.5 Timing properties}

The timing properties of the MWPC are determined by the collection time of the electrons produced by the ionizing track. Depending upon where the charge has been deposited, one can distinguish three regions $\mathrm{A}, \mathrm{B}$ and $\mathrm{C}$, as shown in Figure B.4 [27].

The electrons produced in region A are quickly collected and the ones 


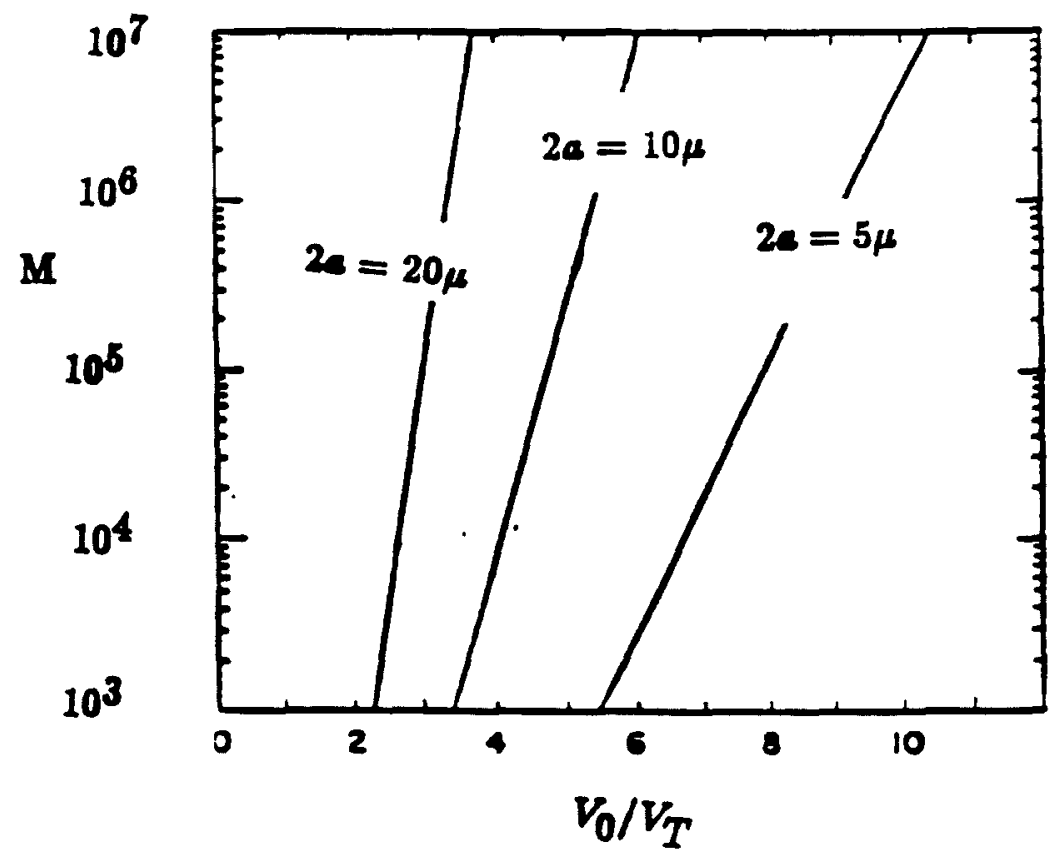

Figure B.3: Multiplication factor as a function of $V_{0} / V_{T}$ 


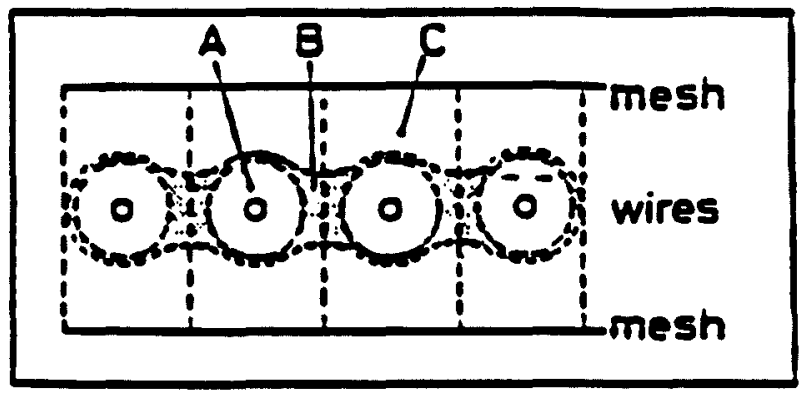

Figure B.4: MWPC timing properties 
produced in region $\mathrm{B}$ are collected at a slower pace. The ones in region $\mathrm{C}$ are collected over a still longer time. The timing distribution of these charge collections are shown in Figure B.5:27].

A gate is defined as the amount of time over which the chamber is allowed to collect the charge from one event. The time resolution of a chamber is the minimum gate necessary for full efficiency.

\section{B.6 Energy loss due to electromagnetic interactions}

The average differential energy loss (in $\frac{M e V}{c m}$ ) due to Coulomb interactions has been obtained by Bethe and Bloch in the frame work of relativistic quantum mechanics, and can be written in the electrostatic unit system as $[27]:$

$$
\begin{gathered}
\frac{d E}{d X}=-K \frac{Z}{A} \frac{\rho}{\beta^{2}}\left[\ln \left(\frac{2 m c^{2} \beta^{2} E_{M}}{I^{2}\left(1-\beta^{2}\right)}-2 \beta^{2}\right)\right] \\
K=\frac{2 \pi N Z^{2} e^{4}}{m c^{2}}
\end{gathered}
$$

where:

$\mathrm{N}=$ Avogadro's number

$\mathrm{m}=$ electron mass

$\mathrm{e}=$ electron charge

$\mathrm{Z}=$ atomic number of the medium

$A=$ atomic mass number of the medium

$\rho=$ density of the medium

$\mathrm{I}=$ effective ionization potential 


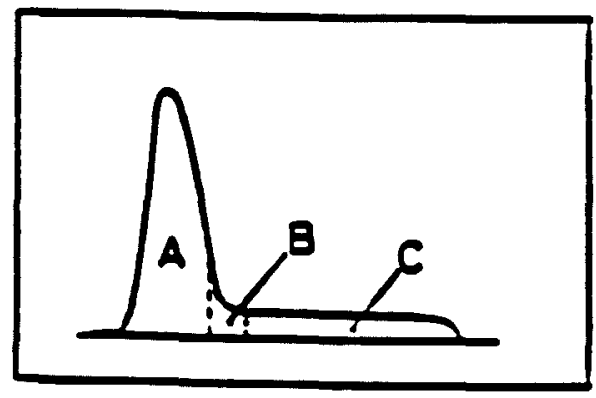

Figure B.5: Timing distribution of charge collection 
$\beta=$ velocity of the projectile in units of speed of light

$\mathrm{K}=0.154 \mathrm{MeV} \mathrm{cm}^{2} / \mathrm{gram}$ for unit charge projectiles

$\mathrm{E}_{M}=$ maximum energy transfer allowed in each interaction

rest energy of the electron $\left(m c^{2}\right)=0.511 \mathrm{MeV}$

Two-body relativistic kinematics gives:

$$
E_{M}=\frac{2 m c^{2} \beta^{2}}{1-\beta^{2}}
$$

One can define a quantity called the reduced length $(x)$, where

$$
x=X \rho
$$

Then:

$$
\frac{d E}{d x}=\frac{1}{\rho} \frac{d E}{d X}
$$

The value of the effective ionization potential $I$ is in general the result of a measurement for each material. A good approximation is that $I=I_{0} Z$, where $I_{0}=12 \mathrm{eV}$.

From Equation B.23, it follows that the differential energy loss depends only on the projectile's velocity $(\beta)$ and not on its mass. After a fast decrease the energy loss reaches a constant value around $\beta=0.97$. This region of constant energy loss is called the minimum ionizing region. For most materials $d E / d x=1.5 \mathrm{MeV} /\left(\mathrm{gram} / \mathrm{cm}^{2}\right)$ at minimum ionization. At higher energies, the energy loss slowly increases as $\beta$ approaches 1 . However then polarisation effects produce a saturation of the energy lost at a value about $50 \%$ above the minimum ionizing region. 
The charged particles which are expected to be detected using the MWPC's in E706 have energy of the order of $10 \mathrm{GeV}$. Hence their energy loss while going through the MWPC is quite negligible compared to their energy.

\section{B.7 Gas used for MWPC}

The electromagnetic energy loss is the result of a small number of discrete interactions. There are two types of such interactions. The first one is close collisions with large energy transfers resulting in liberation of electronic charges. This is called ionization. The second one is distant collisions involving smaller energy transfers resulting in both ionization and atomic excitation. The ionization and excitation share the available energy loss more or less equally.

The avalanche multiplication of charges occurs in noble gases at much lower fields than in complex molecules. This is a consequence of many nonionizing energy dissipation modes available in polyatomic molecules. For the detection of minimum ionizing particles argon is the most suitable gas; but at gains above $10^{3}$ an argon-operated chamber goes into permanent discharge operation for the following reasons [27]. During the avalanche process excited and ionized atoms are formed. The excited noble gas can return to the ground state only through a radiative process. The minimum energy of the emitted photon is well above the ionization potential of the usual cathode materials. Therefore photoelectrons can be extracted from the cathode and they can initiate a secondary avalanche soon after the 
primary one. Argon ions migrate to the cathode and are neutralized by extracting electrons. The energy released in this process is radiated as a photon or used in extracting photoelectrons from the cathode surface. Both these processes result in spurious secondary avalanches.

In polyatomic molecules the secondary emission of electrons is very unlikely, since they have a large number of rotational and vibrational nonradiative states when they contain more than four atoms. This facilitates the absorption of photons in a wide energy range. These molecules dissipate the excess energy from the absorption, either by elastic collisions or by dissociation into simpler radicals. This also happens when a polyatomic ionized molecule neutralizes at the cathode. In the neutralization the radicals recombine either into simpler molecules by dissociation or into larger complexes by polymerization. The quenching efficiency of a polyatomic gas increases with the number of atoms in the molecule. Isobutane is usually used for high-gain stable operation.

The addition of small quantities of electronegative gases like freons allows one to obtain the highest possible gains before the Geiger-Müller discharge. These gases have photon-quenching capability and capture free electrons forming negative ions that cannot induce avalanches. If the mean free path for electron capture is shorter than the distance from anode to cathode, the secondary avalanches described above will not reach the anode and hence will not be detected. To preserve the detection efficiency of the primary avalanche, only limited amounts of electronegative gases can be used. 
Usage of non-polymerizing quenchers like alcohols, aldehydes and acetates would suppress the aging effect: but they have a lower vapor pressure compared to hydrocarbons and this affects the efficiency of quenching against photoionization and secondary avalanches. The solution to this problem is to choose a non-polymerizing agent whose ionization potential is lower than those of other constituents of the gas mixture and to make use of the ion exchange machanism. As a result ions neutralized at the cathode would become of the non-polymerizing species.

A gaseous mixture called "modified magic gas" was used for the MWPC's of E706. This mixture contains argon-freon-isobutane-isopropyl alcohol in the volume proportions of $79.7 \%-0.1 \%-18.0 \%-2.2 \%$. 


\section{Bibliography}

[1] Akesson, T. et al., CERN-EP/89-98 (1989)

[2] Akesson, T. et al., Phys. Lett. 123B (1983) 367

[3] Aurenche, P et al., Phys Lett. 140 B (1984) 87

[4] Alhof, M et al., Part. and Fields 22(1984) 307.

[5] Benson, R., PhD Thesis, University of Minnesota (1990)

[6] Berger, E. L. et al., Nucl. Phys. B239 (1984) 52

[7] Bonesini, M. et al., The structure of events triggered by direct photons in $\pi^{-} \mathrm{p}, \pi^{+} \mathrm{p}$ and pp collisions at $280 \mathrm{GeV} / \mathrm{c}$, Proceedings of the XIV International Symposium on Lepton Photon Interactions, Stanford, (1989)

[8] Bonesini, M. et al., A comparison of hadronic final states in high $p_{T}$ direct photons and $\pi^{0}$ production with those in $e^{+} e^{-}$interactions, $\mathrm{Z}$. Phys. Z44 (1989) 71 (1989)

[9] Brun R., CERN DD (1986)

[10] Charpak G., Ann.Rev.Nucl.Sci.20 (1970) 195 
[11] Constantini, F., Proceedings of the 22nd International Conference of High Energy Physics, Leipzig, (1984)

[12] Contogouris, A. P., Mc Gill University preprint, (1989)

[13] Contogouris, A. P., et al., Phys. Rev. D32 (1985) 1134

[14] De, K. et al., Proceedings of the XXIV Rencontre de Moriond, Les Arcs, (1989)

[15] De Barbaro, L., F Lobkowicz, E706 Internal Note (E706-167) (1988)

[16] DeSoi, W. et al., A Large Liquid Argon Photon/Hadron Calorimeter, Proceedings of the Santa Fe Meeting of the APS, (1984)

[17] DeSoi, W., PhD Thesis, University of Rochester (1990)

[18] Engels, E. Jr. et al., Nucl. Instr. and Meth. A279 (1989) 272

[19]. Ferbel, T. and Molzon, W., Rev. Mod. Phys. 56 (1984) 181

[20] Gutierrez, P. et al., E706 Liquid Argon Calorimeter, Proceedings of the International Europhysics Conference on High Energy Physics, Bari, (1986)

[21] Lobkowicz, F. et al., A Large Liquid Argon Photon/Hadron Calorimeter at FNAL, Nucl. Instr. and Meth. A235 (1985) 332

[22] Mani, S. et al., A Silicon Microstrip Vertex Detector for Direct Photon Physics, Proceedings of the Meeting of the APS, DPF, Eugene (1985). 
[23] Mansour, J., PhD Thesis, Rochester University (1990)

[24] Owens, J. F., FSU HEP. 820315 (1982)

[25] Owens, J. F., Rev.Mod.Phys 59 (1987) 465

[26] Paige, F. E. and Protopopescu, S. D. BNL 38774 (1987)

[27] Sauli F., "Principles of operation of Multiwire Proportional and Drift chambers," Lectures given in the Academic Training Program of CERN , 1975-6 CERN 7209 (1977)

[28] Sinanidis,A., PhD Thesis, Northeastern University. (1989)

[29] Slattery, P. et al., E706 Proposal. (1981)

[30] Trippe T. , "Minimum Requirements for Charpak Chamber wires," CERN NP Internal Report, 69-18 (1969).

[31] Weerasundara, P. P. D. S., Private communication (1989)

[32] Yosef, C., PhD Thesis, Northeastern University (1990)

[33] Zielinski, M., Z. Phys c16 (1983) 197. 